\title{
Evidence of Dopant Type-Inversion and Other Radiation Damage Effects of the CDF Silicon Detectors
}

\author{
Roberto Martínez Ballarín \\ Universidad del País Vasco, \\ Departamento de Ingeniería Nuclear y Mecánica de Fluidos

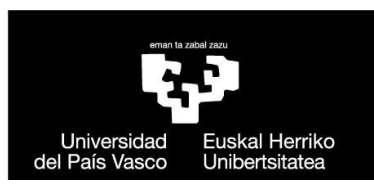

Junio de 2010

directores:

Oscar González López, Ignacio Redondo Fernández, CIEMAT Avenida Complutense 22, 28040 Madrid Madrid, Spain

ponente:

Fernando Legarda Ibáñez, U. del País Vasco

Barrio Sarriena s/n, 48940 Leioa

Vizcaya, Spain. 
(C) Servicio Editorial de la Universidad del País Vasco Euskal Herriko Unibertsitateko Argitalpen Zerbitzua

ISBN: 978-84-694-5817-4 


\section{Acknowledgment}

The author is sincerely thankful to the Ciemat CDF group: Juan Pablo Fernandez, Oscar Gonzalez, and Ignacio Redondo, for its example of professionalism, diligence and enthusiasm. The appreciation is extensive to the Ciemat Departament of Basic Research, for their support and trust.

Also deserving gratitude are The Fermi National laboratory researchers and staff, in particular the Silicon Detector Group where the main work related to this thesis has been done. The close contact with scientist and technicians of this laboratory has been an exceptional opportunity of learning and training, most often beyond science. 



\section{Contents}

List of Figures $\quad$ vii

$\begin{array}{ll}\text { List of Tables } & \text { xvii }\end{array}$

1 Introduction 1

2 Semiconductor Detectors Under Radiation 3

2.1 Radiation Damage in Semiconductors . . . . . . . . . . . . . 3

2.1.1 The Formation of Primary Lattice Defects . . . . . . . . . . . 4

2.1.1.1 Dependence on Radiation Type ............. . 5 5

2.1.1.2 Scaling of Radiation Damage ............ 5

2.1.2 Formation and Properties of Stable Defects . . . . . . . . . . . 5

2.1.3 Electrical Properties of Defect Complexes $\ldots \ldots \ldots$

2.1.3.1 Defects with a Single Entry Level . . . . . . . . . . 9 9

2.1.3.2 Defects with several Energy Levels . . . . . . . . . . . . 13

2.1.3.3 Thermal equilibrium relations ............. 14

2.1.4 Effects of Deffects on Detector Properties . . . . . . . . . . . . . 15

2.1.4.1 Operating Voltage of Detectors . . . . . . . . . 16

2.1.4.2 Reverse-Bias Current ............... 18

2.2 Annealing of Radiation Damage $\ldots \ldots \ldots \ldots$

2.2.1 Beneficial Annealing $\ldots \ldots \ldots \ldots$

2.2.2 Reverse Annealing. . . . . . . . . . . . . . . 21

2.3 Parametrization of Radiation Damage. . . . . . . . . . . . . . 22 
2.4 Mitigation Techniques . . . . . . . . . . . . . . . . 23

2.5 Semiconductors in Particle Physics . . . . . . . . . . . . . . 26

2.5.1 Silicon Particle Detectors . . . . . . . . . . . . . . 27

2.5.2 Vertex and Tracking Detectors at Hadron Colliders . . . . . . . 28

2.5.2.1 CDF and DO at the Tevatron . . . . . . . . . . 28

2.5.2.2 ATLAS and CMS at the LHC . . . . . . . . . . 28

3 CDF at Fermilab 33

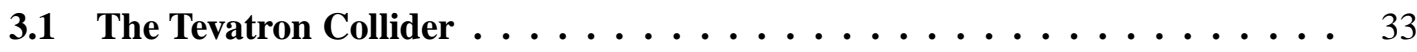

3.2 CDF Run II detector . . . . . . . . . . . . . . . . . . 37

3.2.1 Tracking and Time of Flight systems $\ldots \ldots \ldots \ldots$

3.2.2 Calorimeter system ....................... 41

3.2.3 Muons system . . . . . . . . . . . . . . . . 44

3.3 Luminosity Measurement . . . . . . . . . . . . . . . . . 44

3.3.1 CLC detector .................................. 44

3.3.2 Measurement of the luminosity $\ldots \ldots \ldots$. . . . . . . . . 44

3.4 Trigger and Data Acquisition . . . . . . . . . . . . . 46

3.4.1 Level 1 trigger . . . . . . . . . . . . . . . . 48

3.4.2 Level 2 trigger . . . . . . . . . . . . . . . . . . 49

3.4.3 Level 3 trigger . . . . . . . . . . . . . . . . . . 49

$3.5 \quad B$ Physics with Silicon Detectors $\ldots \ldots \ldots \ldots$

3.5.1 Features of $B$ Physics at a Hadron Collider . . . . . . . . . . . 50

3.5.1.1 Triggering on $B$ Decay Products . . . . . . . . . . . 51

3.5.2 Selected $B$ Physics Results from the Tevatron . . . . . . . . . . . 52

3.5.2.1 $\quad B$ Hadron Masses and Lifetimes . . . . . . . . . . . . 52

3.5.2.2 Prompt Charm Cross Section . . . . . . . . . . 53

3.5.2.3 Hadronic Branching Ratios ............... 53

3.5.2.4 Rare Decays ......................... 54

3.5.2.5 $B_{s}$ Lifetime Difference and Mixing Phase . . . . . . . . 55

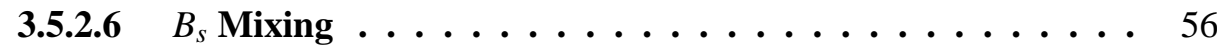


4 The CDF Silicon Detectors

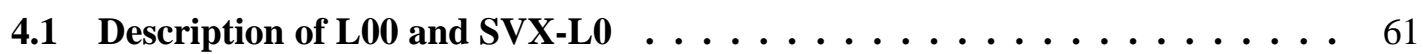

4.1.1

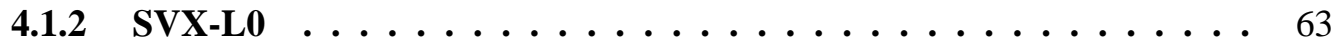

4.2 Radiation enviroment $\ldots \ldots \ldots \ldots \ldots$

4.3 Mitigation of the Radiation Effects . . . . . . . . . . . . . . . 66

5 Leakage Current Analysis $\quad 69$

5.1 Bias scheme . . . . . . . . . . . . . . . . . . . 69

5.1.1 CAEN PS .......................... 70

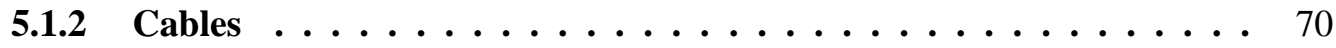

5.1 .3 Junction cards . . . . . . . . . . . . . . . . 71

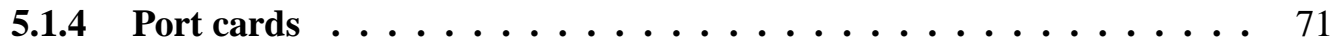

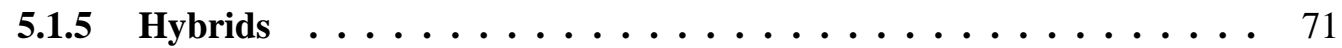

5.1.6 Bias resistors on the detector $\ldots \ldots \ldots \ldots \ldots \ldots \ldots \ldots$

5.1.7 Total resistance computation . . . . . . . . . . . 76

5.2 Radiation Damage Quantification $\ldots \ldots \ldots \ldots \ldots$

5.3 Bias current data $\ldots \ldots \ldots \ldots \ldots \ldots \ldots \ldots$

5.4 Analysis of the bias currents $\ldots \ldots \ldots \ldots$

5.5 Total Bias Current $\ldots \ldots \ldots \ldots \ldots \ldots \ldots$

5.6 Luminosity to Fluence Conversion . . . . . . . . . . . . . . . . . 86

6 Signal vs. Bias Scans and Detector Lifespan Extrapolations $\quad 87$

6.1 Bias voltage scans. The signal vs. bias scan implementation . . . . . . . . 87

6.2 Results of the LO0 Signal vs. Bias scans _ . . . . . . . . . . . . . . . . 89

6.2.1 Study of the Depletion Voltage . . . . . . . . . . . . . . 89

6.2.2 Evolution of the Depletion Voltage . . . . . . . . . . . . . . 92

6.2.3 L00 Extrapolations Summary . . . . . . . . . . . . . 97

6.3 Results of the SVX-LO Signal vs. Bias scan _ . . . . . . . . . . . 100

6.3.1 Study of the Depletion Voltage . . . . . . . . . . . . . 100 
6.3.2 Evolution of the Depletion Voltage . . . . . . . . . . . . . . . 101

6.3.3 SVX-L0 Extrapolations Summary . . . . . . . . . . . . . . . 106

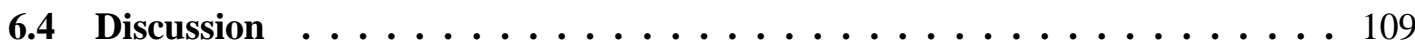

7 Description of radiation aging effects in L00 and SVX-II detectors 111

7.1 Monitoring of detector parameters . . . . . . . . . . . . . 111

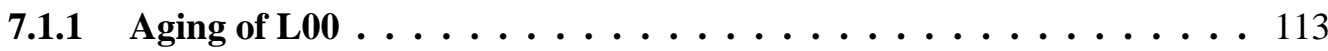

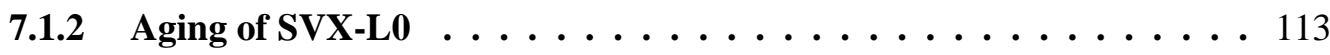

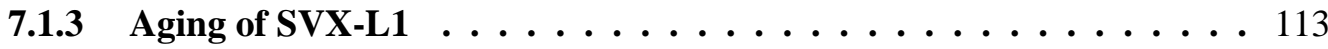

7.2 Summary of parameters by layer . . . . . . . . . . . . . 118

7.3 Behavior at low bias voltage $\ldots \ldots \ldots \ldots \ldots$

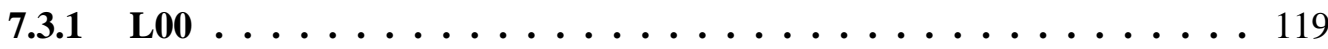

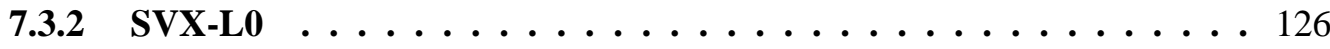

7.3.3 Summary by layer . . . . . . . . . . . . . . . . . . 135

7.3.4 Behavior at low bias voltage and dopant profile models $\ldots \ldots$. . . 137

8 Conclusions 139

A Resumen en Castellano 141

A.1 El experimento CDF en el Laboratorio Federal Fermi . . . . . . . . . . . 141

A.2 Materiales de estado sólido para la detección de radiación ionizante. . . . . . 141

A.2.1 Detectores de vértices de silicio: el detector SVX de microbandas de

CDF. . . . . . . . . . . . . . . . . . . . . 143

A.2.2 Daños por radiación. . . . . . . . . . . . . . . 145

A.3 Aplicaciones a la Física de Altas Energías. . . . . . . . . . . . . . . 145

A.3.1 Importancia de los detectores de vértices de silicio. . . . . . . . . 145

A.3.2 Espectroscopía de hadrones. . . . . . . . . . . . . . . . 146

A.3.3 Búsquedas. . . . . . . . . . . . . . . . . . . 147

A.4 Exploraciones de voltaje de polarización. Exploración de señal vs. polarización. . . . . . . . . . . . . . . . . . . . . . 148

A.4.1 Resultados de las exploraciones de señal vs. polarización para L00. 149 
A.4.1.1 Voltaje de desertización . . . . . . . . . . . . . 149

A.4.1.2 Evolución del voltaje de desertización . . . . . . . . . 152

A.4.1.3 Resumen de extrapolaciones lineales de LOO . . . . . . . 155

A.4.2 Resultados de las exploraciones de señal vs. polarización para SVX-L0.157

A.4.2.1 Voltaje de desertización . . . . . . . . . . 157

A.4.2.2 Evolución del voltaje de desertización $\ldots$. . . . . . . . 159

A.4.2.3 Resumen de extrapolaciones lineales de SVX-LO $\ldots 159$

A.4.3 Discusión . . . . . . . . . . . . . . . . . . 162

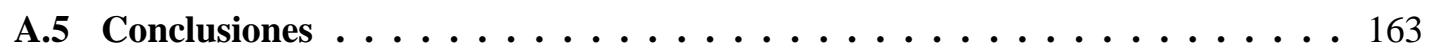

$\begin{array}{lll}B & \text { The diode Equation } & 167\end{array}$

B.1 Carrier concentrations in pure semiconductors . . . . . . . . . . . 168

B.2 Carrier concentrations in doped crystals $\ldots \ldots \ldots \ldots \ldots$

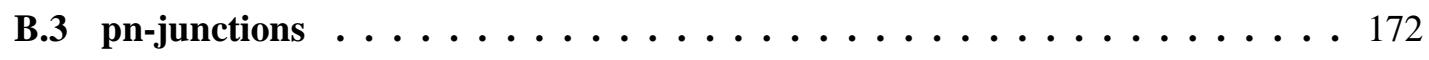

B.4 The forward-biased pn-junction $\ldots \ldots \ldots \ldots \ldots \ldots \ldots$

C Electrical Properties of Impurities and Defects 179

C.1 Emission and capture processes $\ldots \ldots \ldots \ldots$. . . . . . . . . 180

C.1.1 Electron capture . . . . . . . . . . . . . . . . . . . 181

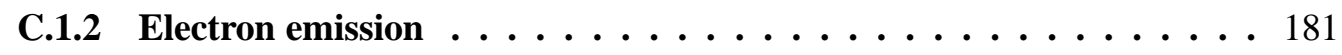

C.1.3 Hole capture . . . . . . . . . . . . . . . . . . 182

C.1.4 Hole emission . . . . . . . . . . . . . . . . . 182

C.1.5 Emission probabilities $\ldots \ldots \ldots \ldots \ldots$. . . . . . . . . 182

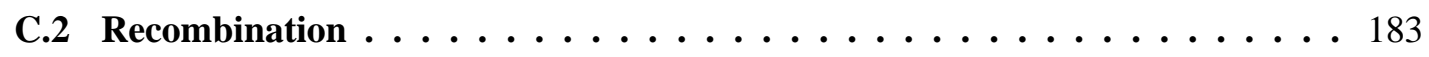

C.2.1 Band-to-band recombination . . . . . . . . . . . . . . 183

C.2.2 Recombination via intermediate states . . . . . . . . . 183

C.3 Carrier generation . . . . . . . . . . . . . . . . . . 185

C.3.1 Generation in the depletion region . . . . . . . . . . . . 185

C.3.2 Generation in the neutral region . . . . . . . . . . . . 186

C.4 The origin of recombination and generation centers $\ldots \ldots \ldots$ 
C.5 The diode equation revisited $\ldots \ldots \ldots \ldots \ldots$

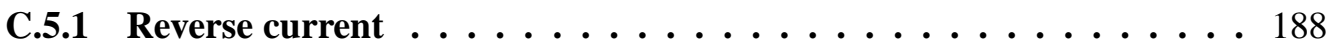

C.5.2 Forward current . . . . . . . . . . . . . . . . . . . . 189

C.5.3 Comments . . . . . . . . . . . . . . 190

D Type Inversion and Doping Profile 193

D.1 Double junction models . . . . . . . . . . . . . . . . . . . . . . . . 194

D.2 Junction models and experimental data . . . . . . . . . . . . . . 197

$\begin{array}{ll}\text { Bibliography } & 207\end{array}$ 


\section{List of Figures}

2.1 A vacancy-donor (Phosphorus atom) complex. A mobile vacancy drifts in the n-type material. It can reach stability besides a donor nucleus, the defect becoming permanent, and it may change the electrical properties of the semiconductor. . . . . . . . . . . . . . . . .

2.2 Energy dependence of non-ionizing energy loss (NIEL) in silicon for various types of radiation $[92] \ldots \ldots \ldots \ldots \ldots$

2.3 Characteristics of some important defects in silicon [71]. . . . . . . . . 8

2.4 Calculated (left) space-charge concentration $v s$. high-energy proton fluence for silicon with initial donor concentrations $N_{d 0}$ of $10^{12}$ and $10^{13} \mathrm{~cm}^{-3}$. With an infinitely fine calculation grid both distributions would dip to zero[70]). Fluence dependence (right) of the magnitude of the effective doping for an $n$-type silicon wafer irradiated with $1 \mathrm{MeV}$ neutrons equivalent. The data have been corrected for self-annealing occurring already during the extended irradiation period. Also shown is the much smaller effect of irradiation with $1.8 \mathrm{MeV}$ electrons also scaled to $1 \mathrm{MeV}$ neutron equivalent NIEL ([71]). . . .

2.5 Bulk n-type damage. Divacancy, disordered region, interstitial atom and Ecenter. . . . . . . . . . . . . . . . . . 17

2.6 Fluence dependence of leakage current for silicon detectors produced by various process technology from different silicon materials. The current was treated after a heat treatment for 80 min at $60^{\circ} \mathrm{C}$ (see Ref. [25]). . . . . . . 30

2.7 Evolution of beneficial annealing $\Delta N_{a}$ and anti-annealing $\Delta N_{Y} v s$. time at $20^{\circ} \mathrm{C}$ and $-5{ }^{\circ} \mathrm{C}$ after proton fluence burst of $10^{14} \mathrm{~cm}^{-2} \ldots \ldots \ldots 31$

3.1 The Tevatron Collider Chain at Fermilab . . . . . . . . . . . . . . . . 34

3.2 Tevatron Collider Run II Integrated Luminosity $\ldots \ldots \ldots$

3.3 Tevatron Collider Run II Peak Luminosity $\ldots \ldots \ldots$ 
3.4 Isometric view of the CDF Run II detector $\ldots \ldots \ldots$

$3.5 r \times \eta$ side view of the CDF Run II detector $\ldots \ldots \ldots \ldots$

3.6 Layout of wire planes on a COT endplate . . . . . . . . . . . . . 39

3.7 Layout of wires in a COT supercell . . . . . . . . . . . . . . . 40

3.8 The CDF II tracker layout showing the different subdetector systems $\ldots$. . . 41

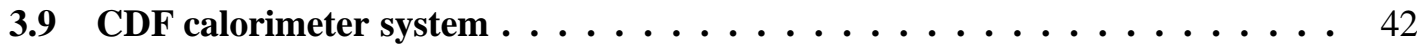

3.10 Block diagram showing the global trigger and DAQ systems at CDF II . . . . 46

3.11 Block diagram showing the Level 1 and Level 2 trigger system _ . . . . . 47

4.1 The CDF detector. The direction of advance of the protons determines the positive cartesian axis $z$. Its innermost system is the silicon detector (L00+SVXII+ISL). Outside are the Central Outer Tracker (COT), the electromagnetic and hadronic calorimeters (EM cal, HAD cal) and the muon chambers. . . . . . . . . . . . 60

4.2 Longitudinal section of CDF and detection ranges for the several subdetectors. The figure shows the first quadrant of the cartesian $Y Z$ plane. The $\eta$ coordinate (pseudo-rapidity) is a transformation of the polar angle $\theta$ under the formula $\eta=-\ln (\tan (\theta / 2)) \ldots \ldots \ldots \ldots$

4.3 Typical lenght between collision vertex and $B$ hadron decay is $L_{x y} \sim 200 \mu \mathrm{m}$. CDF is able to reconstruct vertexes with resolution of about $30 \mu \mathrm{m}$ for high energy particles where multiple scattering can be neglected. . . . . . . . . 61

4.4 Transverse section of the silicon detector showing the three subsystems: LOO, SVX-II and ISL. . . . . . . . . . . . . . . . . . . . 62

4.5 Detail of strips and biasing polysilicon resistors (black blocks) for a LOO SGS Thomson sensor. . . . . . . . . . . . . . . . . . . 64

4.6 Transverse section of L00 (left), and SVX-II (right) whose innermost layer is SVX-Lo. . . . . . . . . . . . . . . . . . 65

4.7 Transverse sections of $\mathrm{LOO}$ and coordinates of its sensors. . . . . . . . . . 65

4.8 Radiation field of CDF. Component of beam losses (left) and collisions. . . . . 66

5.1 Silicon detector bias circuit. . . . . . . . . . . . . . . . . 70

5.2 Outer Junction Card circuit. . . . . . . . . . . . . . . . 72

5.3 Port Card circuit. . . . . . . . . . . . . . . . 73

5.4 Port Card analog section. . . . . . . . . . . . . . . 73 
5.5 Bias current data of $\mathrm{LOO}$ sensor f803. Original (left) and temperature corrected at $20^{\circ} \mathbf{C}$ (right) . . . . . . . . . . . . . . . . . 79

5.6 Vertex position (x-coordinate,on the left; $y$-coordinate, on the right) vs. lu-

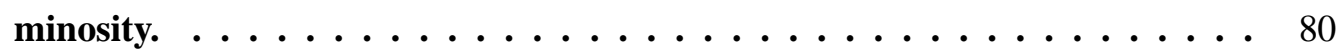

5.7 Example of sliding-fits slopes for sensor f820 (left) and sliding-fits slopes versus distance from the vertex to the sensor (right) . . . . . . . . . . . . 81

5.8 Total bias current measured on narrow sensors (left) and wide sensors (right). 82

5.9 Region 1: zoom of Fig.5.8 in the range from 0 to $1000 p b^{-1} \ldots \ldots \ldots$

5.10 Region 2: zoom of Fig.5.8 in the range from 1000 to $2500 \mathrm{pb}^{-1} \ldots \ldots \ldots$

5.11 Region 3: zoom of Fig.5.8 in the range from 4000 to $5000 \mathrm{pb}^{-1} \ldots \ldots$. . . . 84

5.12 Region 4: zoom of Fig.5.8 in the range from 5500 to $7000 \mathrm{pb}^{-1} \ldots \ldots$. . . . 85

6.1 Example of the signal vs. bias scan method. This scan was performed on L00 at an integrated luminosity of $3512 \mathrm{pb}^{-1}$. The figure on the left shows the charge collection distribution (at the polarization voltage of 10 Volts below the nominal operating voltage) corresponding to one of the 48 modules of L00, and the Landau fit, characterized by its peak or Most Probable Value (MPV). The figure on the right displays the 25 MPVs corresponding to the 25 different polarization voltages. This set of points is fitted to a sigmoid whose $95 \%$ of its height, $\mathbf{7 5 . 6}$ volts, is defined as the depletion voltage for this particular sensor and luminosity. The depletion voltage is the output point of the signal vs. bias scan method. . . . . . . . . . . . . . 88

6.2 Depletion Bias plots for Micron class sensor f813 $\ldots \ldots \ldots \ldots$

6.3 Depletion Bias plots for SGS Thomson sensor f843 . . . . . . . . . . . . . 90

6.4 Depletion Bias plots for Hamamatsu sensor f821 . . . . . . . . . . . . . 91

6.5 Depletion Bias plot for Hamamatsu sensor f841 . . . . . . . . . . . . 91

6.6 Example of inversion of the Micron class sensors f813 and fa43 (luminosity

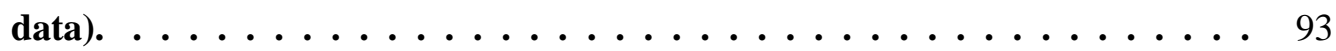

6.7 Example of inversion of the Micron class sensors f813 and fa43 of LOO (fluence data). . . . . . . . . . . . . . . . . . . . 93

6.8 Example of inversion for SGS Thomson sensors f843 and fa13 (luminosity

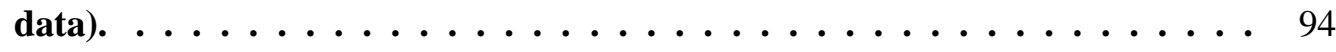

6.9 Example of inversion for SGS Thomson sensors f843 and fa13 (fluence data). 94 
6.10 Example of inversion for Hamamatsu sensors f821 and fa31 (luminosity data). 95

6.11 Example of inversion for Hamamatsu sensors f821 and fa31 (fluence data). . 95

6.12 Example of inversion for Hamamatsu sensors f841 and fa11 (luminosity data). 96

6.13 Example of inversion for Hamamatsu sensors f841 and fa11 (fluence data). . 96

6.14 Global results for L00 luminosity plots. Summary of extrapolation fits of L00 at latest luminosity of $6888 \mathrm{pb}^{-1}$ (left), and at previous luminosity of $4530 \mathrm{pb}^{-1}$ (right). . . . . . . . . . . . . . . . . 97

6.15 Global results for L00 fluence plots. Summary for L00 sensors of abscises of the inversion point (left) and linear fits slopes (right). Geometrical interpretation is altered to include the Micron sensors ( $f * 3)$. Coordinates of this sensors lie in the plane corresponding to columns of $z=1$ and $z=8 \ldots \ldots$. . . 98

6.16 Distribution of the coordinates of the inversion point for LOO sensors (luminosity version). Left, $x$-coordinate; right, $y$-coordinate. The existence of two peaks of populations is expected since different classes of L00sensors invert at different points. . . . . . . . . . . . . . . . 98

6.17 Distribution of the $x$-coordinate of the inversion point (left) and linear fit slope for points after inversion (right) (fluence version) for L00 sensors (fluence version). . . . . . . . . . . . . . . . . .

6.18 Global results for L00 and SVX-L0 luminosity plots. Comparison of inversion luminosities for the different classes of sensors of LOO and SVX-LO. Results were computed after data taken at a latest luminosity of $6097 \mathrm{pb}^{-1}$. . 99

6.19 Depletion voltage for $\phi$ (left) and $z$ (right) sides of e160 ladder at $6888 \mathbf{p b}^{-1}$. . 100

6.20 Depletion voltage for $\phi$ (left) and $z$ (right) sides of e460 ladder at $6888 \mathrm{pb}^{-1} \ldots 101$

6.21 Method of noise vs. bias scan. This method is valid only for double-sided sensors. . . . . . . . . . . . . . . . . . . . . 102

6.22 Signal vs. bias and noise vs. bias combined results for sensors e160 and e460. The noise scan method is only reliable for the region before type-inversion. 103

6.23 Historical evolution of depletion voltage for sensor e160 (luminosity). . . . 104

6.24 Historical evolution of depletion voltage for sensor e160 (fluence). . . . . . . 104

6.25 Historical evolution of collected charge in full-depletion mode for ladder e160, $\phi$ (left) and $z$ (right) sides. . . . . . . . . . . . . . 105 
6.26 SVX-L0 time evolution data over type-inversion prediction model. Dots are the 3 modules for which early signal vs. bias scan data is available. . . . . . 105

6.27 Extrapolation fits for all the SVX-L0 sensors' $\phi$ sides at 6888 (left) and $4550 \mathrm{pb}^{-1} .107$

6.28 Extrapolation fits for all the SVX-L0 sensors' $z$ sides at 6888 (left) and $4550 \mathrm{pb}^{-1} .107$

6.29 Global results for SVX-L1 luminosity plots. Summary of extrapolation fits for all the SVX-L1 sensors' $p h i$ sides (left) and $z$ sides (right), at $6888 \mathrm{pb}^{-1}$. Almost flat slopes in SVX-L1 evidence that this layer has not clearly suffered type inversion. . . . . . . . . . . . . . . . . . . 108

6.30 Global results for SVX-L0 fluence plots. Summary for SVX-LO sensors of the linear fits slopes for $p h i$ sides (left) and $z$ sides (right). . . . . . . . . . . 108

6.31 Left: calculated space charge vs. high-energy proton fluence for silicon with initial donor concentrations $N_{d 0}$ of $10^{12}$ and $10^{13} \mathrm{~cm}^{-3}$. Whith an infinitely fine calculation grid both distributions would dip to zero (Ref. [70]). Right: experimental data of the inversion curve from CDF (LOO module fa13). The fact that the minimum of this curve does not reach to zero volts is in conflict with the model. . . . . . . . . . . . . . . . . . . . 110

7.1 Example of RMS and cluster size (number of strips) distributions and fitting functions at bias voltage of $\mathbf{- 5}$ volts with respect to the nominal for $\mathrm{LO0}$ sensor f843. The Lorentzian (on the the residuals distributions) and Landau (on the cluster fits distribution) fits are plotted to guide the eye. . . . . . . . . . . 112

7.2 Four detector variables as a function of bias voltage for $\mathrm{LO0}$ sensor f843. The fits on the right plot did not converge for this particular detector. . . . . . . . 112

7.3 Evolution of the four detector variables for L00 sensor f843. . . . . . . . . 114

7.4 Historical plot of charge and efficiency for SVX-LO sensor e030, phi (left) and $\mathrm{z}$ sides. . . . . . . . . . . . . . . . . . . . 115

7.5 Historical plot of cluster size and resolution for SVX-LO sensor e030, phi (left) and $z$ sides. . . . . . . . . . . . . . . . . . . . . . 115

7.6 Historical plot of charge and efficiency for SVX-L1 sensor e031, phi (left) and $\mathrm{z}$ sides. . . . . . . . . . . . . . . . . . . . 116

7.7 Historical plot of cluster size and resolution for SVX-L1 sensor e031, phi (left) and $z$ sides. . . . . . . . . . . . . . . . . . . . . . 116

7.8 Ideal SVX-L0 sensor space charge (homogeneous dopant profile) and signal vs. bias voltage curves behavior along luminosity. . . . . . . . . . . 138 
7.9 Real SVX-LO sensor space charge (inhomogeneous dopant profile, double junction like) and signal vs. bias voltage curves behavior along luminosity. The presence of a slope $s$ in the experimental curves could be an evidence of double-junction dopant profile structure (Appendix D). . . . . . . . . . . . 138

A.1 Detector CDF. La dirección de avance de los protones determina el sentido positivo de la coordenada cartesiana $z$. El detector más interno es el de silicio (L00+SVXII+ISL) y hacia el exterior se sitúan la cámara central de trazas (Central Outer Tracker,COT), los calorímetros electromagnético y hadrónico (EM cal, HAD cal) y las cámaras de muones. . . . . . . . . . . . . . . 142

A.2 Sección transversal del detector de silicio. Se muestran los tres subsistemas que lo integran: LO0, SVX-II e ISL. . . . . . . . . . . . . . . . . . . . . 144

A.3 Sección longitudinal de CDF y rangos de detección para los distintos subdetectores. Se muestra el primer cuadrante del plano cartesiano $Y Z$. La coordenada $\eta$ (pseudo-rapidez) es una transformación del ángulo polar $\theta$ según la fórmula $\eta=-\operatorname{Ln}(\operatorname{tg}(\theta / 2)) . \ldots \ldots \ldots \ldots$

A.4 Vértices de colisión. $L_{x y} \sim 200 \mu \mathrm{m} \ldots \ldots \ldots \ldots \ldots \ldots \ldots$. . . . . . . . 148

A.5 Ejemplo del método de exploración de señal vs. polarización. Esta prueba se practicó para L00 a una luminosidad integrada de $3512 \mathrm{pb}^{-1}$. La figura de la izquierda muestra la distribución de la colección de carga (a un voltaje de polarización de 10 voltios por debajo del voltaje nominal de operación del sensor) correspondiente a uno de los 48 modulos de LOO y su curva de ajuste (landau) caracterizada por su valor más probable (VMP). La figura de la derecha muestra el conjunto de 25 VMPs correspondiente a los 25 voltajes de polarización. Este conjunto de puntos se ajusta mediante una curva sigmoide cuyo $95 \%$ del valor de su altura, 75.6 voltios, se considera como el voltaje de desertización de este particular sensor y para la luminosidad de $3512 \mathrm{pb}^{-1}$. Este valor del voltaje de desertización es el resultado del método de exploración de señal vs. polarización. . . . . . . . . . . . . . . . . . 149

A.6 Figuras de voltaje de desertización para el sensor f813 de clase Micron de L00. Se observan pequeños incrementos en el voltaje de desertización para este sensor desde la última prueba a $4530 \mathrm{pb}^{-1}$, un resultado esperable dado el especial diseño de estos sensores. . . . . . . . . . . . . . . . . 150

A.7 Figuras de voltaje de desertización para el sensor f843 de clase SGS Thomson de LO0. . . . . . . . . . . . . . . . . . . . . . 150 
A.8 Figuras de voltaje de desertización para el sensor f821 de clase Hamamatsu de LO0. . . . . . . . . . . . . . . . . . . . 151

A.9 Figuras de voltaje de desertización para el sensor f841 de clase Hamamatsu de LO0. . . . . . . . . . . . . . . . . . . . . . . 151

A.10 Ejemplo de inversión para los dos sensores de clase Micron de LO0. $\ldots \ldots$

A.11 Ejemplo de inversión para dos sensores de clase SGS Thomson de L00. . . 153

A.12 Ejemplo de inversión para dos sensores de clase Hamamatsu de L00. . . . . . 154

A.13 Ejemplo de inversión para dos sensores de clase Hamamatsu de L00. . . . . . 154

A.14 Resultados generales para L00 y SVX-L0. Comparación de la luminosidad de inversión para las diferentes clases de sensores de LO0 y SVX-LO. Los resultados se computaron con datos tomados a una luminosidad de $6097 \mathrm{pb}^{-1} .155$

A.15 Figura resumen para L00. Ajustes lineales superpuestos de todos los sensores de L00. Datos a 6888 pb $^{-1}$ (izquierda), y a 4530 pb $^{-1}$ (derecha). . . . . . . 156

A.16 Voltaje de desertización para las caras $\phi$ (izquierda) y $z$ (derecha) del sensor e160 a luminosidad de $6888 \mathrm{pb}^{-1}$. . . . . . . . . . . . . . . 157

A.17 Voltaje de desertización para las caras $\phi$ (izquierda) y $z$ (derecha) del sensor e460 a luminosidad de $6888 \mathrm{pb}^{-1} \ldots \ldots \ldots \ldots$. . . . . . . . . . . . . . . . . . .

A.18 Evolución histórica del voltaje de desertización del sensor e160 de SVX-L0, cara $\phi$ (izquierda) $\mathbf{y} z$ (derecha) $\ldots \ldots \ldots$

A.19 Evolución histórica de la carga colectada del sensor e160 de SVX-L0, caras $\phi$ (izquierda) y $z$ (derecha). . . . . . . . . . . . . . . . 160

A.20 Datos de evolución histórica de SVX-L0 superpuestos al modelo predictivo de inversión de tipo de dopante. Los puntos corresponden a los 3 sensores para los que existe estadística disponible a bajas luminosidades. . . . . . . 160

A.21 Resumen de la extrapolación de los ajustes lineales para las caras phi de los sensores de SVX-L0, a 6888 pb $^{-1}$ (izq.) y $4530 \mathrm{pb}^{-1} \ldots \ldots \ldots$. . . . . . . . . 161

A.22 Resumen de la extrapolación de los ajustes lineales para las caras $z$ de los sensores de SVX-L0, a 6888 pb $^{-1}$ (izq.) y $4530 \mathrm{pb}^{-1} \ldots \ldots \ldots$. . . . . . . . 161

A.23 Espacio de carga de un sensor de SVX-L0 ideal (perfil de dopante homogéneo) y comportamiento frente a luminosidad de la curva de señal vs. polarización. 163 
A.24 Espacio de carga de un sensor de SVX-L0 real (perfil de dopante inhomogéneo, caso de lineal simple) y comportamiento frente a luminosidad de la curva de señal vs. polarización. La presencia de la pendiente $s$ observada en las curvas experimentales podría evidenciar una estructura subyacente de doble unión (Apéndice D). . . . . . . . . . . . . . . . . . . . . . . . . . . 164

C.1 Emission and capture processes through intermediate states. The arrows show the direction of electron transitions. . . . . . . . . . . . . . . . 181

C.2 Direct Transition (a), and Indirect Transition, via an intermediate state (b). The arrows show the direction of electron transitions. Direct transitions are extremely improbable in Silicon ("indirect bandgap"). In both cases it is supposed that the generation phase is due to an external irradiation and the bias voltage is zero (equilibrium, $\mathbf{V}$ bias=0). . . . . . . . . . . . . 183

C.3 Carrier generation and recombination probabilities. The width of the arrows gives an idea of the probability of each transition. The mid-gap case (the defect energy is that of the intrinsic semiconductor) is preferred for a sequential double transition. For the generation figure (up) it is supposed a reverse bias $(\mathrm{V}<0)$ in order to swept the carriers away, and not considering their recombinations. For the recombination figure (down) the generation phase is supposed to be created by a forward bias $(\mathrm{V}>0)$ ( or an external radiation with bias $\mathrm{V}=0$ ) . . . . . . . . . . . . . . . . 19

D.1 Left: calculated space charge vs. high-energy proton fluence for silicon with initial donor concentrations $N_{d 0}$ of $10^{12}$ and $10^{13} \mathrm{~cm}^{-3}$. Whith an infinitely fine calculation grid both distributions would dip to zero (Spieler, 2005). Right: Fluence dependence of the magnitude of the effective doping for an $n$ type silicon wafer irradiated with $1 \mathrm{MeV}$ neutrons equivalent. The data have been corrected for self-annealing occurring already during the extended irradiation period. Also shown is the much smaller effect of irradiation with 1.8 MeV electrons also scaled to 1MeV neutron equivalent NIEL (Lutz, 1999). 194

D.2 Model and simulation of clustered defects. . . . . . . . . . . . . 195

D.3 EVL model. . . . . . . . . . . . . . . . . . . . . . . . . 197

D.4 Type inversion experimental data. Example of two L00 opposite sensors: f843 and fa13. . . . . . . . . . . . . . . . . . . 198 
D.5 Inhomogeneous Model (double pn-junction). The linear distribution of the original inhomogeneous model could be replaced by a trigonometric distribution. . . . . . . . . . . . . . . . . . . . . 204

D.6 Electronic circuit superimposed over a cross section of the sensor. The detector's bulk is represented as a capacitor in the circuit. When an incident particle ionizes the silicon, the detector's bulk circuit is transformed into a current source in parallel, to account the creation of electron-hole pairs. In a capacitive coupling (made by capacitor $C$ ) design, only the $\mathrm{AC}$ part of the detector reverse bias current $I_{r}$ reaches the readout and amplifying electronics. The DC part goes into a bias circuit, here shown as a simple resistor $R$. Intermediate "floating" electrodes can be used to reduce the effective readout pitch. In this case, the signal current induced on the floating electrodes is transferred to the readout amplifiers by intermediate capacitive dividers formed by the strip-to-strip capacitance $C_{s s}$ and the backplane capacitance $C_{b} .205$ 



\section{List of Tables}

3.1 Accelerator parameters for Run II configuration . . . . . . . . . . . . 35

3.2 CDF II Calorimeter subsystems and characteristics $\ldots \ldots \ldots$. . . . . . . 43

4.1 Naming convention and detector properties for Layer 00 and the five layers of SVX II . . . . . . . . . . . . . . . . . . . . . . . 64

5.1 Technical parameters of $\mathrm{LOO}$ sensors. Last row contains the polysilicon biasing resistor values needed for the total resistance computation. . . . . . . . . 74

5.2 Technical parameters of SVXII sensors. Eighth row contains the polysilicon biasing resistor values needed for the total resistance computation. . . . . . . 75

5.3 Summary of technical parameters of LOO and SVXII sensors needed for the total resistance computation. . . . . . . . . . . . . . . . . 77

7.1 Evolution of the efficiency vs. luminosity (red graph) along z-axis for selected modules of LO and L1. . . . . . . . . . . . . . . . . . . . . 117

7.2 Global values per layer. Average over total number of sensors of detector's parameters versus luminosity. . . . . . . . . . . . . . . . 118

7.3 Type-inversion evolution for L00-f850, example of Hamamatsu class sensor (wide). Inversion point at $944 \mathrm{pb}^{-1}$. First row contains the turn-on curves of the collected charge and efficiency set of points. In the second row is displayed the first derivative of the red sigmoid fitting the charge points of first row. Third row is reserved for the number of strips and the residuals. . . . 120 
7.4 Type-inversion evolution for L00-f843, example of SGS Thomson class sensor (standard narrow). Inversion point at $2041 \mathrm{pb}^{-1}$. First row contains the turn-on curves of the collected charge and efficiency set of points. In the second row is displayed the first derivative of the red sigmoid fitting the charge points of first row. Third row is reserved for the number of strips and the residuals. . . . . . . . . . . . . . . . . 12

7.5 Type-inversion evolution for L00-f813, example of Micron radiation-hardened class sensor (oxygenated narrow). Inversion point at $2494 \mathrm{pb}^{-1}$. First row contains the turn-on curves of the collected charge and efficiency set of points. In the second row is displayed the first derivative of the red sigmoid fitting the charge points of first row, and an analogous black curve for the black sigmoid curve that fits the region of low voltages in the first row. Third row is reserved for the number of strips and the residuals. . . . . . . . . . . . . 122

7.6 Collected charge, efficiency, cluster size and residuals evolution for the three classes of LO0 sensors. . . . . . . . . . . . . . . . . . . . . 123

7.7 Type-inversion evolution for L00-f813, example of Micron radiation-hardened class sensor (oxygenated narrow). Inversion point at $2494 \mathrm{pb}^{-1}$. All luminosities part I. Black plot is a fit for low bias voltages (limit of the black fit:

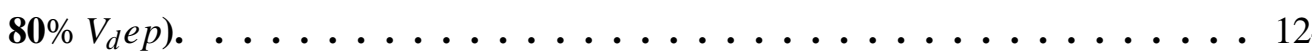

7.8 Type-inversion evolution for L00-f813, example of Micron radiation-hardened class sensor (oxygenated narrow). Inversion point at $2494 \mathrm{pb}^{-1}$. All luminosities part II. Black plot is a fit for low bias voltages (limit of the black fit:

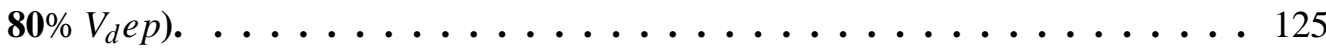

7.9 Type-inversion evolution for L0-e090, a Hamamatsu class sensor. Inversion point at $1902 \mathrm{pb}^{-1}$. Each one of the three columns represents a stage point in the lifespan of the sensor ( $p h i$ and $z$ sides are shown): before, after and at inversion. The red sigmoid fits the whole set of charge points while the black sigmoid fits only the subset of points at low voltages. Second row holds the first derivative of those curves, respecting the color code. . . . . . . . . . 127

7.10 Type-inversion evolution of cluster size and residuals for L0-e090, a Hamamatsu class sensor. Inversion point at $1902 \mathrm{pb}^{-1} \ldots \ldots \ldots$. . . . . . . . 128 
7.11 Type-inversion evolution for L0-e140, a Hamamatsu class sensor. Inversion point at $1902 \mathrm{pb}^{-1}$. Each one of the three columns represents a stage point in the lifespan of the sensor ( $p h i$ and $z$ sides are shown): before, after and at inversion. The red sigmoid fits the whole set of charge points while the black sigmoid fits only the subset of points at low voltages. Second row holds the first derivative of those curves, respecting the color code. . . . . . . . . . . 129

7.12 Type-inversion evolution of cluster size and residuals for L0-e140, a Hamamatsu class sensor. Inversion point at $1902 \mathrm{pb}^{-1} \ldots \ldots \ldots$. . . . . . . . 130

7.13 Type-inversion evolution for L0-e2a0, a Hamamatsu class sensor. Inversion point at $1902 \mathrm{pb}^{-1}$. Each one of the three columns represents a stage point in the lifespan of the sensor ( $p h i$ and $z$ sides are shown): before, after and at inversion. The red sigmoid fits the whole set of charge points while the black sigmoid fits only the subset of points at low voltages. Second row holds the first derivative of those curves, respecting the color code. . . . . . . . . . 131

7.14 Type-inversion evolution of cluster size and residuals for L0-e2a0, a Hamamatsu class sensor. Inversion point at $1902 \mathrm{pb}^{-1} \ldots \ldots \ldots$. . . . . . . . 132

7.15 Type-inversion evolution for L0-e2a0. All luminosities, phi side. Black plot is a fit for low bias voltages (limit of the black fit: 80\% $V_{d}$ ep). . . . . . . . 133

7.16 Type-inversion evolution for L0-e2a0. All luminosities, $z$ side. Black plot is a fit for low bias voltages (limit of the black fit: 80\% $V_{d}$ ep). . . . . . . . 134

7.17 Low-voltage behavior of L00 sensors: ordinate at origin of low-voltages sigmoid fit vs luminosities for the three classes of LO0 sensors. . . . . . . . . 135

7.18 Low-voltage behavior of SVX-LO sensors: ordinate at origin of low-voltages sigmoid fit vs luminosities for the three sensors of SVX-LO with more statistics. 136

D.1 Parameters of the EVL Model [81]. . . . . . . . . . . . . . . . . . . 196

D.2 Type-inversion evolution models. . . . . . . . . . . . . . . . . 202

D.3 Homogeneous and inhomogeneously distributed space charge models of an irradiated sensor. . . . . . . . . . . . . . . . . 203 



\section{Chapter 1}

\section{Introduction}

The Fermi National Accelerator is an international High Energy Physics reserch center located in Chicago, Illinois (USA). Its facilities house the Tevatron accelerator. The Tevatron accelerates protons and antiprotons up to an energy of $2 \mathrm{TeV}$ in the center of mass and focuses the beams in two interaction points where the detectors DO and CDF are located.

The CDF detector is made up of several subdetectors: muon chambers, calorimeters, wire chamber, silicon detectors... The silicon detectors are dedicated to charged particle tracking and vertex reconstruction and are the closest located devices to the beam, and hence, the most affected by the radiation field caused by the collisions. The silicon detectors are essentially $p$ semiconductor microstrips deposited on a $n$ material bulk $(\approx \mathbf{3 0 0} \mu \mathrm{m}$ thick, typically ), creating an arragement of $p-n$ linear junctions on one side of the sensor. CDF is the largest example so far of double-sided technology, a particular kind of microstrips where both sides are segmented, providing two coordinates.

The aim of this document is to study the effect of radiation damage on the silicon sensors. The reflection of the effect of radiation can be observed in two fundamental parameters of the detector: the bias current and the bias voltage. The leakage current directly affects the noise, while the bias voltage is required to collect the maximum signal deposited by the charged particle.

On one hand, the bias current increases with irradiation. This circumstance can be useful to estimate the radiation dose received by the detector. On the other hand, the depletion voltage (i.e. the bias voltage needed to operate a sensor) evolves with time. Since the silicon detectors are essentially an arrangement of semiconductor $p n$ junctions, an intense and prolonged irradiation over a junction is responsible of the phenomenon known as "dopant type-inversion" where the n-type and p-type silicon forming the junction experience the effect of turning into p-type and n-type, respectively. This inversion is reflected in the evolu- 
tion of the depletion voltage in the form of a curve with a minimum. Extrapolations based on those curves are useful to estimate the sensor's lifespan. The estimation of the lifespan of the sensors has received particular interest since the CDF microstrip detectors have received substantially more irradiation than it was designed for. The large scale of the sensors irradiated $\left(\approx 7 \mathrm{~m}^{2}\right.$ of silicon) and the use in the innermost layers of materials developed for the next generation of LHC silicon sensors, widen the interest range of this study to the High Energy Physics detector community.

The document starts with a general introduction to semiconductor detectors followed by two chapters describing the CDF experiment and the CDF Silicon Detectors. Chapters 5 and 6 are devoted to the study of the two fundamental parameters of the sensors under irradiation: the bias current and the bias voltage. Chapter 7 contains a collection of evidences of radiation aging and also some ideas for future reserch, and the conclusions are shown in chapter 8. 


\section{Chapter 2}

\section{Semiconductor Detectors Under Radiation}

\subsection{Radiation Damage in Semiconductors}

The radiation itself that we want to detect may also cause damage to the detectors. Nuclear radiation interacts with the electron cloud, but also with the nuclei in the lattice. While the interaction with the electron cloud in silicon is a transient effect (that is in fact used for the detection of the radiation), the interaction with the lattice may lead to permanent material changes of detrimental nature. The following processes are of importance for the lattice:

$\star$ Displacement of lattice atoms, leading to interstitials (atoms between regular lattices) and vacancies (empty lattice sites);

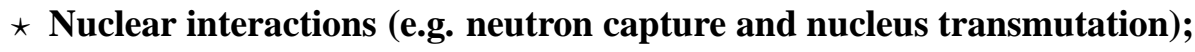

$\star$ Secondary processes from energetic displaced lattice atoms, defect clusters from cascade processes...

Most of these primary defects are not stable. Interstitials and vacancies are mobile at room temperature and will therefore partially anneal if by chance an interstitial fills the place of vacancy. There are also chances for the formation of other room temperature stable defects. Examples are the well known A-center, a combination of vacancy and oxygen (a certain concentration of oxygen interstitials is present in the crystal after crystal growing), the divacancy (two missing silicon atoms right next to each other), and the E-center shown in Fig.2.1, a vacancy-phosphorus complex. These stable defects may then change the electrical properties of the semiconductor, generally degrading them. 

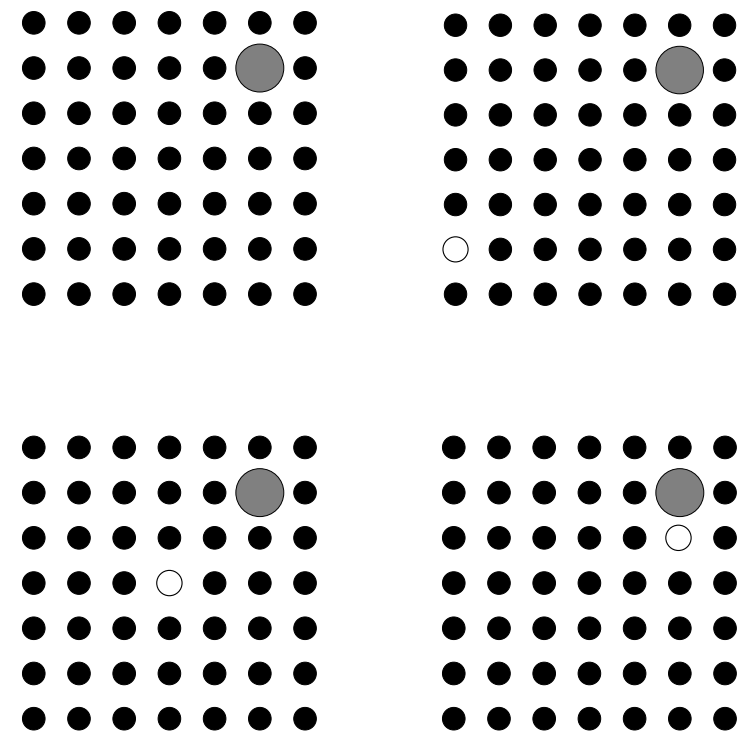

Figure 2.1: A vacancy-donor (Phosphorus atom) complex. A mobile vacancy drifts in the n-type material. It can reach stability besides a donor nucleus, the defect becoming permanent, and it may change the electrical properties of the semiconductor.

\subsubsection{The Formation of Primary Lattice Defects}

A minimum recoil energy of $15 \mathrm{eV}$ displaces a silicon atom from its lattice site. This energy can be provided by elastic scattering of a high-energy charged or neutral particle. For the recoiling atom it is defined the displacement energy $E_{d}$, the energy at which the displacement probability is roughly one half. This recoiling energy is $E_{d}=\mathbf{2 5} \mathrm{eV}$ for silicon (van Lint et al. 1980, Huhtinen 2001) and, depending on values relative to it, the following effects may be created:

$*$ recoil energies below $E_{d}=\mathbf{2 5} \mathrm{eV}$ will predominately lead to lattice vibrations only;

* for recoil energies below roughly 1-2 keV (close above $E_{d}$ ), only isolated point defects will be created;

* between $2 \mathrm{keV}$ and $12 \mathrm{keV}$ the energy is high enough to crate one defect cluster and additional point deffects;

* above $12 \mathrm{keV}$ several clusters and additional point defects will be produced. 
A cluster is a dense agglomeration of point defects that appear at the end of a recoil silicon track where the atom loses its last 5-10 keV of energy and the elastic scattering crosssection increases by several orders of magnitude. A typical size for a cluster is $5 \mathrm{~nm}$ diameter with 100 lattice displacements ${ }^{1}$.

\subsubsection{Dependence on Radiation Type}

The probability for creation of a primary knock-on atom (a silicon atom displaced from its lattice location by the incident radiation) as well as its energy distribution depend on the type and energy of the incident radiation, for the following reasons:

* the elastic cross-section for scattering on silicon atoms depends on the type of radiation. Charged particles such as protons scatter by electrostatic interaction with the partially screened nucleus; neutral particles such as neutrons scatter elastically with the nucleus only.

* the energy transferred to the silicon atom is dependent on the mass of the incident radiation.

\subsubsection{Scaling of Radiation Damage}

Although the primary interaction of radiation with silicon is strongly dependent on the type and energy of the radiation, this dependence is to a large extend smoothed out by the secondary interaction of primary knock-on silicon atoms. It is therefore customary to scale measurements of radiation damage from one type of radiation and energy to another. As the interaction of radiation with electrons produces ionization but no crystal defects, the quantity used for scaling is the non-ionizing energy loss (NIEL). The dependence of this scaling variable on energy and type of irradiation (normalized to $1 \mathrm{MeV}$ neutrons) is shown in Fig. 2.2.

\subsubsection{Formation and Properties of Stable Defects}

The primary defects, Si interstitials and vacancies, are at room temperature still mobile and cannot be considered stable. Part of these defects will anneal either by an interstitial filling

\footnotetext{
${ }^{1}$ Such irradiated silicon surfaces may have catalyst or ionic conductivity properties (useful for example as proton exchange membrane of a FC). At the current fluency, the depth of the bulk silicon layer is too much wide to be percolated by the radiation-made channels and clusters but a test could be done with thinner silicon layers. In the current situation of a wide depth silicon layer with relatively shallow clusters, the dielectric breakdown limit may be reduced with the cluster penetration depth $(\approx 2500 \mathrm{~V}$, not a concern with the current bias voltages $)$ and hence generating a different kind of physical defects (i.e. Linchtenberg figures) also susceptible of having similar utilities.
} 


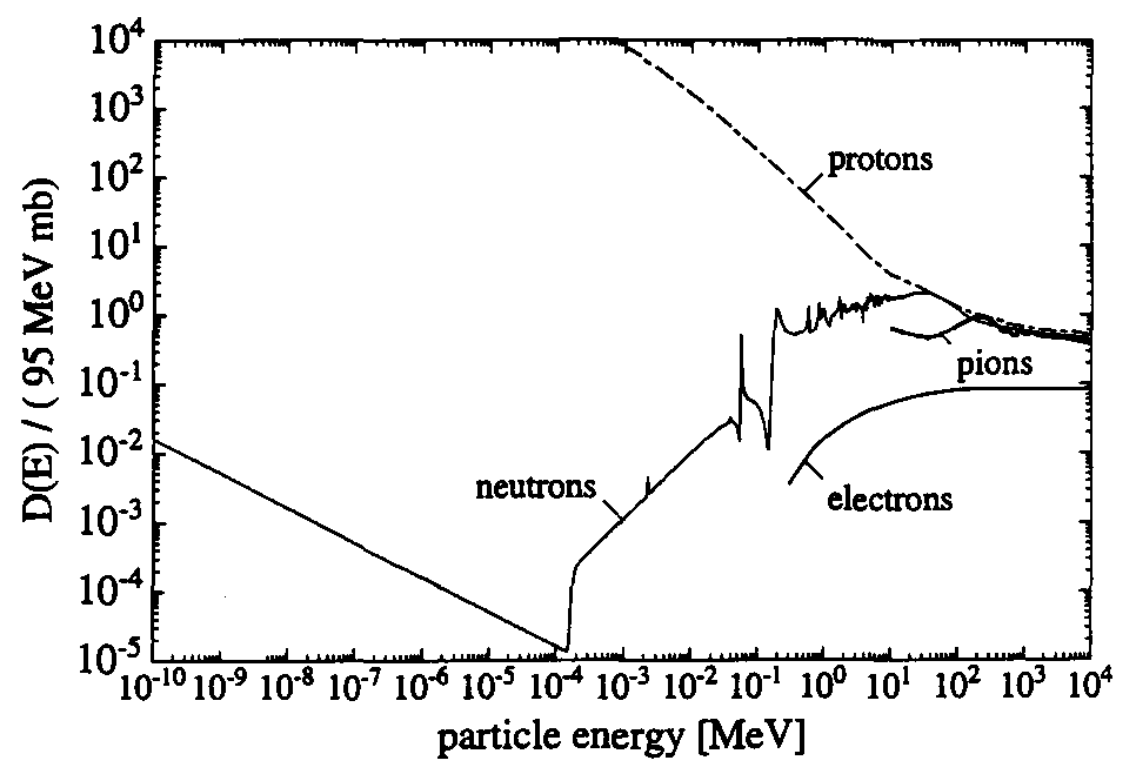

Figure 2.2: Energy dependence of non-ionizing energy loss (NIEL) in silicon for various types of radiation [92]

a vacancy or by diffusing out of the surface. They may, however, also interact with another defect and form a new type of defect complex that becomes stable at room temperature. The other defect may have been already present in the crystal or can also have been produced by the radiation. An example of the first kind of defect is the formation of a vacancyphosphorus complex (E-center). The standard dopant for $n$-type silicon is phosphorus. A vacancy right next to the phosphorus dopant (on a regular lattice site) forms a stable complex with new electrical properties. The phosphorus atom does not fulfill its original role of donor any more, and the process is therefore also called donor removal (Fig. 2.1).

A second example is the formation of vacancy-oxygen complexes (A-center). Oxygen is always present within silicon to a certain degree as a remnant from the crystal-growing process. Oxygen as an interstitial (between regular lattice sites) is electrically inactive. The stable oxygen-vacancy complex becomes electrically active, i.e., it forms an acceptor state in the upper half of the band gap that, although electrically neutral in the space-charge region, acting as a trapping center for electrons.

An example of a stable defect complex that can be produced from radiation-generated primary defects alone is the divacancy, namely two missing silicon atoms right next to each other. The formation of defect complexes in semiconductors is complicated and only par- 
tially understood. Defect complexes are not only produced in two-step processes, as was the case in the examples given above, but processes involving several steps also occur. Important roles in these processes are played not only by the radiation-generated primary defects but also by defects already abundantly introduced during the crystal-growing process. Well known examples in silicon are oxygen and carbon interstitials. The formation processes are also dependent on the temperature. Defect complexes that are stable at room temperatures may become mobile or may even break up into their constituents at elevated temperatures. This opens up the possibility of reducing the apparent radiation damage (annealing) and the formation of defects of a different nature.

The defect complexes change the macroscopic properties of the semiconductor. A large effort has been invested to correlate the macroscopic property changes with specific microscopic defects[24]. Several experimental methods have been invented to recognize the presence of defects and to measure their properties. Most of them are based on creating a thermal disequilibrium and observation of the transition to thermal equilibrium as a function of temperature.

\subsubsection{Electrical Properties of Defect Complexes}

Contrary to simple flat donors and acceptors, which are usually intentionally introduced into regular lattice sites in order to change the properties of the semiconductor, radiationgenerated defect complexes have much more complicated electrical properties. It is the purpose of this section to review the most important of their properties and to discuss the consequences to be expected for detector operation. This includes, in particular, the behavior of defects in the space-charge region. The radiation-generated defects will have the following main consequences:

* They act as a recombinantion-generation centers. They are able to capture and emit electrons and holes. In the space-charge region of a detector, alternate emission of electrons and holes leads to an increase of the reverse-bias current;

* They act as trapping centers. Electrons or holes are captured and re-emitted with some time delay. In the space-charge region of the detector, signal charge is trapped and may be released too late for efficient detection, thus causing a reduction in the signal; and

* They can change the charge density in the space-charge region, thus requiring an increased bias voltage to make the detector fully sensitive.

A list of well known defects and defect complexes is compiled in Table 2.3. It is remarkably that some defects can exist not only in two but frequently in more than two charge states. 


\begin{tabular}{|l|l|l|l|l|}
\hline Impurity & $\begin{array}{l}\text { Charge } \\
\text { state }\end{array}$ & $\begin{array}{l}\text { Energy } \\
\text { level }\end{array}$ & $\begin{array}{l}\text { Charge } \\
\text { in s.c. } \\
\text { region }\end{array}$ & \\
\hline phosphorus & $\begin{array}{l}\mathrm{P}^{0} \\
\mathrm{P}^{+}\end{array}$ & $E_{\mathrm{C}}-0.045$ & + & \\
\hline boron & $\begin{array}{l}\mathrm{B}^{-} \\
\mathrm{B}^{0}\end{array}$ & $E_{\mathrm{V}}+0.045$ & - & \\
\hline
\end{tabular}

\begin{tabular}{|c|c|c|c|c|}
\hline Defect & $\begin{array}{l}\text { Charge } \\
\text { state }\end{array}$ & $\begin{array}{l}\text { Energy } \\
\text { level }\end{array}$ & $\begin{array}{l}\text { Charge } \\
\text { in s.c. } \\
\text { region }\end{array}$ & $\begin{array}{l}\text { Annealing } \\
\text { temp. } \\
T_{\text {ann }}[\mathrm{K}]\end{array}$ \\
\hline interstitial & $\begin{array}{l}\mathrm{I}^{-} \\
\mathrm{I}^{0} \\
\mathrm{I}^{+}\end{array}$ & $\begin{array}{l}E_{\mathrm{C}}-0.39 \\
E_{\mathrm{V}}+0.4\end{array}$ & 0 & $\begin{array}{l}140-180 \\
540-600 \\
370-420\end{array}$ \\
\hline vacancy & $\begin{array}{l}V^{-} \\
V^{-} \\
V^{0} \\
V^{+} \\
V^{++}\end{array}$ & $\begin{array}{l}E_{\mathrm{C}}-0.09 \\
E_{\mathrm{C}}-0.4 \\
E_{\mathrm{V}}+0.05 \\
E_{\mathrm{V}}+0.13\end{array}$ & 0 & $\begin{array}{l}\approx 90 \\
150\end{array}$ \\
\hline divacancy & $\begin{array}{l}V_{2}^{-} \\
V_{2}^{-} \\
V_{2}^{0} \\
V_{2}^{+}\end{array}$ & $\begin{array}{l}E_{\mathrm{C}}-0.23 \\
E_{\mathrm{C}}-0.39 \\
E_{\mathrm{V}}+0.21\end{array}$ & 0 & $\begin{array}{l}\approx 570 \\
\approx 570 \\
\approx 140 \\
\approx 570\end{array}$ \\
\hline A-center & $\begin{array}{l}(\mathrm{V}-\mathrm{O})^{-} \\
(\mathrm{V}-\mathrm{O})^{0}\end{array}$ & $E_{\mathrm{C}}-0.18$ & 0 & $\approx 600$ \\
\hline E-center & $\begin{array}{l}(\mathrm{V}-\mathrm{P})^{-} \\
(\mathrm{V}-\mathrm{P})^{0}\end{array}$ & $E_{\mathrm{C}}-0.44$ & 0 & $\approx 420$ \\
\hline $\begin{array}{l}\text { boron } \\
\text { interstitial }\end{array}$ & $\begin{array}{l}\mathrm{B}_{I}^{-} \\
\mathrm{B}_{I}^{0} \\
\mathrm{~B}_{I}^{+}\end{array}$ & $\begin{array}{l}E_{\mathrm{C}}-0.45 \\
E_{\mathrm{C}}-0.12\end{array}$ & 0 & 420 \\
\hline $\begin{array}{l}\text { vacancy } \\
\text { boron }\end{array}$ & $\begin{array}{l}(\mathrm{V}-\mathrm{B})^{0} \\
(\mathrm{~V}-\mathrm{B})^{+}\end{array}$ & $E_{\mathrm{V}}+0.45$ & 0 & $\approx 300$ \\
\hline $\begin{array}{l}\text { vacancy } \\
\text { arsenic }\end{array}$ & $\begin{array}{l}(\mathrm{V}-\mathrm{As})^{-} \\
(\mathrm{V}-\mathrm{As})^{0}\end{array}$ & $?$ & $?$ & 440 \\
\hline
\end{tabular}

Figure 2.3: Characteristics of some important defects in silicon [71]. 


\subsubsection{Defects with a Single Entry Level}

Let us start with a simple donor, a defect that can be positively charged or neutral depending (in thermal equilibrium) on the position of the Fermi level. The simplest example for a donor is the replacement of a silicon atom by phosphorus with one more electron in the outer shell. The additional electron may either be bound to the phosphorus atom (occupied or neutral defect state) or emitted to the conduction band (empty or positively charged defect state). The charge state of the defect is changed not only by electron emission to the conduction band and electron capture, but it may also be changed from positive to neutral by hole emission to the valence band or from neutral to positive by hole capture. The charge state of the defect $(\mathrm{P}$ in $\mathrm{Si})$ changes via the four charge-changing processes:

$* e^{-}$emission to the conduction band: from NEUTRAL to POSITIVE, probability of $e^{-}$emission: $\varepsilon_{n}$.

$* e^{-}$capture from the conduction band: from POSITIVE to NEUTRAL, $e^{-}$capture rate: $R_{n}$.

$* h^{+}$emission to the valence band: from POSITIVE to NEUTRAL, probability of $h^{+}$ emission: $\varepsilon_{p}$.

$* h^{+}$capture from the valence band: from NEUTRAL to POSITIVE, $h^{+}$capture rate: $R_{p}$.

The average electron occupation probability for the donor will be determined by the probability of the four charge-changing processes. Although we have a priori no knowledge on the individual probabilities - they should be determined experimentally- it is possible to find relationships between them from thermal equilibrium considerations. In thermal equilibrium the occupation probability $F$ is given by temperature and Fermi level as

$$
F\left(E_{d}\right)=\frac{1}{1+e^{\frac{E_{d}-E_{F}}{k T}}}
$$

with $E_{d}$ the defect energy level, $E_{F}$ the Fermi level, $k$ the Boltzmann constant and $T$ the temperature. For reasons of simplicity we do not consider here and in the following the degeneration of charge states, an aspect taken into account correctly when generalizing to defects with several energy states. In thermal equilibrium the rates of electron emission and electron capture have to be equal as there is no net flow of electrons to or from the conduction band. The same is true for hole capture and emission. For electrons, the capture rate $R_{n}$ will be proportional to the number of unoccupied donors $\left(1-F\left(E_{d}\right)\right) N_{d}$ and to the electron concentration $n=n_{i} e^{\frac{E_{F}-E_{i}}{k T}}$. Being, for electrons, $\sigma_{n}$ the capture cross section and 
$v_{t h n}$ the thermal velocity, we have

$$
R_{n}=n N_{d}(1-F) \sigma_{n} v_{t h n}=\sigma_{n} v_{t h n} n_{i} N_{d}(1-F) e^{\frac{E_{F}-E_{i}}{k T}} .
$$

The electron generation rate $G_{n}$ will be given by the product of density of occupied defects $N_{d} F$ and the electron emission probability $\varepsilon_{n}$. Thus

$$
G_{n}=N_{d} F \varepsilon_{n}
$$

Setting the two rates equal to one another, in thermal equilibrium, we have

$$
R_{n}=G_{n} \Rightarrow \varepsilon_{n}=\sigma_{n} v_{t h n} \frac{1-F}{F} n_{i} e^{\frac{E_{F}-E_{i}}{k T}}
$$

where

$$
\frac{1-F}{F}=e^{\frac{E_{d}-E_{F}}{k T}}
$$

so obtaining

$$
\varepsilon_{n}=\sigma_{n} v_{t h} n_{i} e^{\frac{E_{d}-E_{i}}{k T}}
$$

a relationship not containing the Fermi level and valid also under nonequilibrium conditions. Similarly for the hole capture rate and probability of hole emission, we have

$$
\begin{gathered}
R_{p}=p N_{d} F \sigma_{p} v_{t h p}=\sigma_{p} v_{t h p} n_{i} N_{d} F e^{\frac{E_{i}-E_{F}}{k T}} \\
\varepsilon_{p}=\sigma_{p} v_{t h p} n_{i} e^{\frac{E_{i}-E_{d}}{k T}} .
\end{gathered}
$$

These electron and hole capture and emission probabilities can be used to find the interesting physical quantities as average charge state, current generation and trapping probability also in nonequilibrium situations such as in the space-charge region of a detector.

The phosphorus donor has an energy level $0.045 \mathrm{eV}$ below the conduction band (Table in Fig 2.3). Assuming it to be first in the neutral state, it may become ionized (positively) by emitting an electron into the conduction band. A minimum energy of $0.045 \mathrm{eV}$ is necessary for this process. The donor state can come back to neutral again by either capturing an electron from the conduction band or by emitting a hole of minimum energy $E_{g}-\mathbf{0 . 0 4 5} \mathrm{eV}$ into the valence band.

In thermal equilibrium a donor state will be on average half the time in the charged state and half the time in the neutral state when the Fermi level $E_{F}$ coincides with the donor energy level $E_{D}$. If the Fermi level is only a few times the thermal energy $(k T=0.025 \mathrm{eV}$ at room temperature) above the donor level, it will be almost permanently neutral; if it is a few times $k T$ below the donor level, the state will be almost permanently positively charged.

Considering now the situation in the space-charge region of a depleted detector, the Fermi level loses its significance as we are not dealing with equilibrium any more. Instead, 
we have to use the physically significant quantities of electron and hole capture and emission probabilities. For low reverse-bias currents we may assume negligible electron and hole densities within the space-charge region ${ }^{2}$ and ignore electron and hole capture, so that we have to consider emission process only. The ratio of emission and hole probabilities

$$
\frac{\varepsilon_{n}}{\varepsilon_{p}}=\frac{\sigma_{n} v_{t h n}}{\sigma_{p} v_{t h p}} e^{\frac{2\left(E_{d}-E_{i}\right)}{k T}}
$$

is strongly dependent on the energy level of the defect, while the absorption cross-sections and thermal velocities of electrons and holes will be of similar magnitude. We can therefore conclude that defects with a single energy level located above the band gap center $E_{i}$ (i.e. donors) have much higher emission probability for electrons than for holes. The defect will therefore be predominately in the more positive state. Analogously, defects with energy levels below $E_{i}$ (i.e. acceptors) will be in the more negative state when located in the spacecharge region.

Emission Processes. In order to find the average charge state of a single energy level $\left(E_{d}\right)$ defect in the space-charge region, let us consider the donors and acceptors above and below the intrinsic level $E_{i}$. Let us assume that the product of capture cross-section and thermal velocity is the same for electrons and holes $\left(\sigma_{n}=\sigma_{p}, v_{t h n}=v_{t h p}\right)$. We call $E_{d}$ the energy level, $N_{d}$ the density, $f_{1}$ the fraction of defects being in the more negative state, and $f_{0}=1-f_{1}$ the fraction in the more positive state. In the space-charge region the density of electrons and holes is close to zero so that capture processes can be neglected and only electron and hole emission have to be considered. The number of electrons and holes emitted per unit volume and unit time has to be equal, and we have therefore:

$$
N_{d} f_{1} \varepsilon_{n}=N_{d} f_{0} \varepsilon_{p}
$$

which, with Eq.(2.8) and the short notation

$$
c_{n}=v_{t h n} \sigma_{n} \quad \text { and } \quad c_{p}=v_{t h} \sigma_{p}
$$

yields to

$$
\frac{f_{1}}{1-f_{1}}=\frac{\varepsilon_{p}}{\varepsilon_{n}}=\frac{c_{p}}{c_{n}} e^{\frac{-2\left(E_{d}-E_{i}\right)}{k T}}
$$

and so

$$
\begin{gathered}
f_{1}=\frac{1}{1+\frac{c_{n}}{c_{p}} e^{\frac{2\left(E_{d}-E_{i}\right)}{k T}}} \\
f_{0}=1-f_{1}=\frac{1}{1+\frac{c_{p}}{c_{n}} e^{\frac{-2\left(E_{d}-E_{i}\right)}{k T}}} .
\end{gathered}
$$

\footnotetext{
${ }^{2}$ This is not the case for a heavily radiation-damaged silicon detector, a situation with appreciable reverse-bias current.
} 
Measuring charges in units of elementary charge, the average charge that the defect state will assume is the charge of the more positive state $q_{0}$ minus the occupation probability of the more negative state $f_{1}$, thus:

$$
\left\langle q_{d}\right\rangle=q_{0}-f_{1}=q_{1}+f_{0},
$$

and $f_{0}$ will change from one to zero, $f_{1}$ from zero to one within a few multiples of $k T$ around $E_{i}$ when the defect level is moved from the lower to the upper half of the band gap. Therefore only defects with energy levels close to $E_{i}$ will be, on average, fractionally charged. The average charge states of donors (which can only be positively charged or neutral) and acceptors are with the assumptions of equal products of cross-section and thermal velocity of electrons and holes $\left(c_{n}=c_{p}\right)$, thus:

$$
\begin{aligned}
& \left\langle q_{D}\right\rangle=f_{0}=\frac{1}{1+e^{\frac{-2\left(E_{D}-E_{i}\right)}{k T}}} \\
& \left\langle q_{A}\right\rangle=-f_{1}=\frac{-1}{1+e^{\frac{2\left(E_{A}-E_{i}\right)}{k T}}}
\end{aligned}
$$

Capture Processes. Let us consider a reversely biased silicon detector at room temperature $(300 K)$ with a high density of bulk defects, such that an appreciable reverse-bias current is present. In this case, capture processes cannot be ignored and the electron and hole concentrations will be a function of position inside the active region of the detector.

Supposing that for a particular position the electron and hole current densities $J_{n}$ and $J_{p}$ as well as the electric field $\varepsilon$ and single-level $\left(E_{d}\right)$ defect density $N_{d}$ are given, it would be interesting to find the average charge state of the defect at that position and see the conditions under which the presence of leakage currents influences the charge state of the defects. The electron and hole densities can be found from the current densities and the electric field as

$$
n=\frac{J_{n}}{q \mu_{n} \varepsilon} \quad p=\frac{J_{p}}{q \mu_{p} \varepsilon},
$$

and setting, for instance, the values $J_{n}=J_{p}=10 \mu \mathrm{A} / \mathrm{cm}^{2}$ and $\varepsilon=1000 \mathrm{~V} / \mathrm{cm}$, leads to

$$
n=4.6 \times 10^{7} \mathrm{~cm}^{-3} \quad p=1.3 \times 10^{8} \mathrm{~cm}^{-3} \quad n_{i}=1.45 \times 10^{10} \mathrm{~cm}^{-3}
$$

Changing from the more positive to the more negative state is accomplished by hole emission and electron capture, so that we can write with the same notation used before:

$$
N_{d} f_{1}\left(\varepsilon_{n}+p c_{p}\right)=N_{d} f_{0}\left(\varepsilon_{p}+n c_{n}\right),
$$

and using (2.1.3.3) and (2.1.3.3), yields

$$
\frac{f_{1}}{f_{0}}=\frac{f_{1}}{1-f_{1}}=\frac{\varepsilon_{p}+n c_{n}}{\varepsilon_{n}+p c_{p}}=\frac{c_{p} n_{i} e^{-\frac{E_{d}-E_{i}}{k T}}+n c_{n}}{c_{n} n_{i} e^{\frac{E_{d}-E_{i}}{k T}}+p c_{p}},
$$


and so

$$
\begin{aligned}
& f_{1}=\frac{c_{p} n_{i} e^{-\frac{E_{d}-E_{i}}{k T}}+n c_{n}}{c_{n} n_{i} e^{\frac{E_{d}-E_{i}}{k T}}+p c_{p}+c_{p} n_{i} e^{-\frac{E_{d}-E_{i}}{k T}}+n c_{n}} \\
& f_{0}=\frac{c_{n} n_{i} e^{\frac{E_{d}-E_{i}}{k T}}+p c_{p}}{c_{n} n_{i} e^{\frac{E_{d}-E_{i}}{k T}}+p c_{p}+c_{p} n_{i} e^{-\frac{E_{d}-E_{i}}{k T}}+n c_{n}}
\end{aligned}
$$

The average charge is again given by Eq.(2.11):

$$
\left\langle q_{d}\right\rangle=q_{0}-f_{1}=q_{1}+f_{0}
$$

Assuming the energy levels above midgap, we can approximate these expressions for the case with $n<<n_{i}, p<<n_{i}, E_{d}>E_{i}, c_{n} \approx c_{p}$, we obtain:

$$
f_{1}=\frac{1}{1+\frac{c_{n}}{c_{p}} e^{\frac{2\left(E_{d}-E_{i}\right)}{k T}}}\left(1+\frac{c_{n}}{c_{p}} \frac{n}{n_{i}} e^{\frac{\left(E_{d}-E_{i}\right)}{k T}}\right) .
$$

For defect energy levels close to the midgap, the average charge state of the defect will be uncharged from that in a true space-charge region. For larger distances the limiting value $f_{1} \rightarrow 0$ is reached with a factor 2 flater exponential slope:

$$
f_{1}=\frac{c_{n}}{c_{p}} e^{\frac{-2\left(E_{d}-E_{i}\right)}{k T}}+\frac{n}{n_{i}} e^{-\frac{E_{d}-E_{i}}{k T}} .
$$

\subsubsection{Defects with several Energy Levels}

To study the case of defects with several possible charge states we select the divacancy (Table in Fig. 2.3) as an example. The divacancy has four possible charge states, ranging from double-negative to single-positive, and three energy levels, two above and one below midgap. Assuming this level to be initially in a neutral state, it may may change to the positive state by capturing a hole, thereby gaining $0.21 \mathrm{eV}$ of thermal energy, or by emitting an electron of energy $E_{G} \mathbf{- 0 . 2 1} \mathrm{eV}$. Alternatively, it may change to a single negatively charged state by capturing an electron or emitting a hole of energy $E_{G}-0.39 \mathrm{eV}$. Another hole emission of energy $E_{G}-\mathbf{0 . 2 3} \mathrm{eV}$ or electron capture brings it to double-negative state. It may turn back to the neutral state by two electron emissions of energies $0.23 \mathrm{eV}$ and $0.39 \mathrm{eV}$ respectively. Each of the charge states corresponds to a particular lattice distortion and corresponding chemical binding. 
The electrical characteristics of a general type $\operatorname{defect}^{3}$ can be described by the following quantities:

$* k$ energy levels $E_{l}$ describing the energy involved in the change between charge states $l-1$ and $l$;

$* k+1$ charge states with charge $q_{0}-l$ (units of elementary charge) and degeneration factors $g_{l}, l=\mathbf{0}, \mathbf{1}, \ldots, k$;

* $k$ electron capture cross-sections $\sigma_{n, l}$ describing the change from charge state $l-1$ to $l$; and

$* k$ hole capture cross-sections $\sigma_{p, l}$ describing the change from charge state $l$ to $l$-1.

As was the case before for simple defects, the electron and hole emission probabilities $\varepsilon_{n, l}$ and $\varepsilon_{p, l}$ (with $l=1, \ldots, k$ ) can be inferred from the capture cross-sections by considering thermal equilibrium conditions as shown below.

\subsubsection{Thermal equilibrium relations}

Fermi statistics will give the probability for finding the defect in a particular charge state. One has for the ratio of probabilities for finding the defect in two neighboring charge states

$$
\frac{P_{t, l} / g_{l}}{P_{t, l-1} / g_{l-1}}=e^{-\frac{E_{t, l}-E_{F}}{k T}}
$$

The sum of the probabilities for finding the defect in any charge state has to be the unity:

$$
\sum_{l=0}^{k} P_{t, l}=1 .
$$

combining the $k$ equations with, it is possible to find the thermal equilibrium probabilities of the $k+1$ charge states.

Thermal equilibrium is kept by continuous change between neighboring charge states due to electron and hole emission and capture. Changing from charge state $l-1$ to $l$ is accomplished by electron capture (capture cross-section $\sigma_{n, l}$ ) or hole emission (hole emission probability $\left.\varepsilon_{p, l}\right)$. Changing in the opposite direction, from $l$ to $l$-1, involves a hole capture cross-section $\sigma_{p, l}$ and an electron emission probability $\varepsilon_{n, l}$. With this definition, the index for capture and emission constants has the range 1 to $k$.

\footnotetext{
${ }^{3}$ We leave aside the possibility that a defect is able to exist in more than one configuration of the same charge but different energy. The situation that several configurations with the same charge and energy exist can be taken into account by the introduction of a degeneration factor $g$.
} 
As was the case for simple defects, thermal equilibrium considerations will give a relationship between emission and absorption. In order to retain constant average probabilities of the defect charge states and to have zero net flow of electrons and holes towards conduction and valence bands, the electron capture rate of charge state $l-1$ has to equal the electron emission rate of charge state $l$. A similar relation holds for holes:

$$
\begin{aligned}
& N_{t} P_{t, l-1} n c_{n, l}=N_{t} P_{t, l} \varepsilon_{n, l} \\
& N_{t} P_{t, l} p c_{p, l}=N_{t} P_{t, l-1} \varepsilon_{p, l}
\end{aligned}
$$

from this expressions and using we can derive the emission probabilities:

$$
\begin{gathered}
\varepsilon_{n, l}=\frac{P_{t, l-1} n c_{n, l}}{P_{t, l}}=\frac{g_{l-1}}{g_{l}} e^{\frac{E_{t, l}-E_{F}}{k T}} n c_{n, l}=\frac{g_{l-1}}{g_{l}} e^{\frac{E_{t, l}-E_{i}}{k T}} n_{i} c_{n, l} \\
\varepsilon_{p, l}=\frac{P_{t, l} p c_{p, l}}{P_{t, l-1}}=\frac{g_{l}}{g_{l-1}} e^{-\frac{E_{t, l}-E_{F}}{k T}} p c_{p, l}=\frac{g_{l}}{g_{l-1}} e^{-\frac{E_{t, l}-E_{i}}{k T}} n_{i} c_{p, l}
\end{gathered}
$$

These expressions are, with the exception of the additionally introduced degeneration factor $g$, identical to and which were derived for simple donors and acceptors.

\subsubsection{Effects of Deffects on Detector Properties}

The presence of radiation-induced crystal defects will change the detector properties in several ways. The main changes are:

* The increase of reverse-bias current;

* The change of space-charge density; and

* The trapping of signal charges.

For a diode strip detector used for position measurement, trapping is of less importance than reverse-bias current and operating voltage of the detector, while for a CCD (ChargeCouple Device) trapping is extremely dangerous due to the signal-transfer mechanism.

In the following sections, a qualitative explanation of these effects will lead to a parametrization of the effects as a function of the equivalent fluence of neutrons with an energy of $1 \mathrm{MeV}$ scaled according to the non-ionizing energy loss (NIEL). The discussion follows closely the first extended systematic study of radiation damage of silicon detectors [93] and concentrates on radiation damage of neutrons to $n$-type silicon. 


\subsubsection{Operating Voltage of Detectors}

There are several radiation-damage mechanisms that lead to a change in space charge and consequently to a charge in the necessary operational voltage of detectors. As it has been shown, the original dopants such as phosphorus or boron may be captured into new defect complexes, thereby losing their original function as flat donors or acceptors. The new defect complexes may assume a charge state within the space-charge region different from the original dopants. Phosphorus, for example, may transform into vacancy-phosphorus (Ecenter), thereby changing from positive to neutral space charge. In addition to complexes involving the original dopants, complexes with other impurities such as oxygen and carbon, as well as with other radiation-generated primary defects such as divacancies, can be formed (Fig. 2.5). Some known defects of these types are included in Table 2.3. This table, however, is significantly incomplete, and much has to be learned from experimental research.

Operationally, the charge in space charge appears as a change in doping level, so the net space charge is commonly referred to as an effective doping level $N_{\text {eff }}$. The detector functions as before and no change in bias polarity is needed, but to transport charge through the full detector thickness $d$ the voltage must be raised proportionally to the increase in space charge:

$$
V=\frac{e}{2 \varepsilon}\left|N_{e f f}\right| d^{2} .
$$

In analogy to conventional diode operation this is often referred to as the "depletion voltage".

The effective doping of an initially $n$-type silicon wafer is shown as a function of the irradiation fluence in Fig 2.4. The effective doping decreases with irradiation and the material becomes intrinsic ${ }^{4}$ at an irradiation fluence of few times $10^{12} \mathrm{n} / \mathrm{m}^{2}$. Above this value the doping becomes effectively $p$-type (type inversion ${ }^{5}$ ) and eventually rises linearly with the fluence ([93]. This behavior matches perfectly the expectation that the following processes are responsible for the effective doping changes:

$\star$ removal of donors due to the formation of defect complexes containing donors (e.g. vacancy-phosphorus complexes)

* removal of acceptors due to the formation of defect complexes containing acceptors (e.g. vacancy-boron complexes)

$\star$ creation of defect complexes assuming positive charge states in the space-charge region (effective 'donors'); and

\footnotetext{
${ }^{4}$ i.e. undoped. The fluence $\Phi$ at which type inversion occurs depends on the original doping concentration.

${ }^{5}$ Note that the phenomenon of type inversion is not associated with the creation of mobile holes, so altough the material appears like as $p$-type, it is not the same as conventional $p$-doped material.
} 
$\star$ creation of defect complexes assuming negative charge states in the space-charge region (effective 'acceptors').

The effect of electrons is much smaller than neutrons even after scaling by NIEL, as shown in Fig. 2.4 (right). The non-holding of NIEL scaling, particularly for electrons, makes extremely difficult to estimate the irradiation without previous knowledge of the fluxes of the different types of particle. Fortunately in hadron colliders as Tevatron the dose is dominated by collision-generated particles (hadrons) that break NIEL scaling in a less dramatic way.
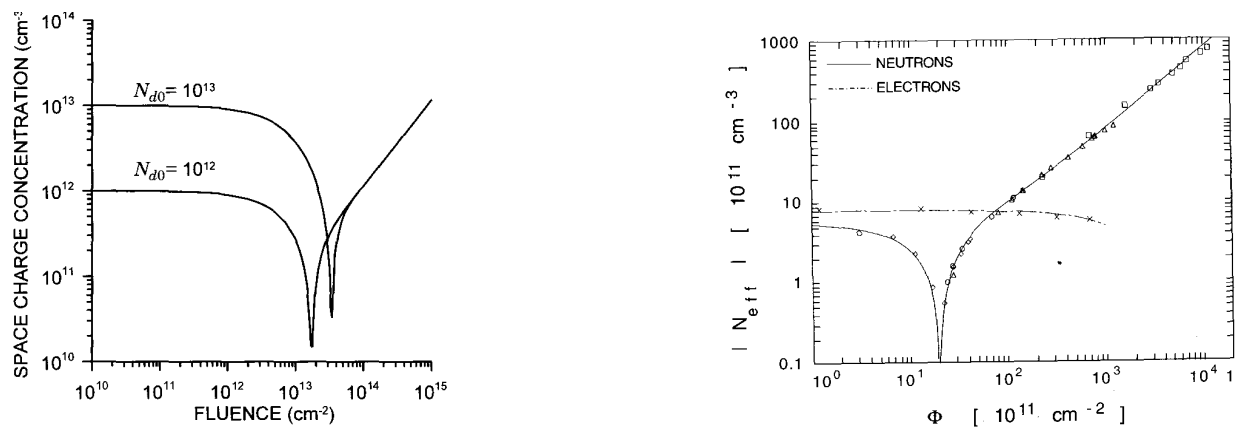

Figure 2.4: Calculated (left) space-charge concentration $v s$. high-energy proton fluence for silicon with initial donor concentrations $N_{d 0}$ of $10^{12}$ and $10^{13} \mathrm{~cm}^{-3}$. With an infinitely fine calculation grid both distributions would dip to zero[70]). Fluence dependence (right) of the magnitude of the effective doping for an $n$-type silicon wafer irradiated with $1 \mathrm{MeV}$ neutrons equivalent. The data have been corrected for self-annealing occurring already during the extended irradiation period. Also shown is the much smaller effect of irradiation with $1.8 \mathrm{MeV}$ electrons also scaled to $1 \mathrm{MeV}$ neutron equivalent NIEL ([71]).

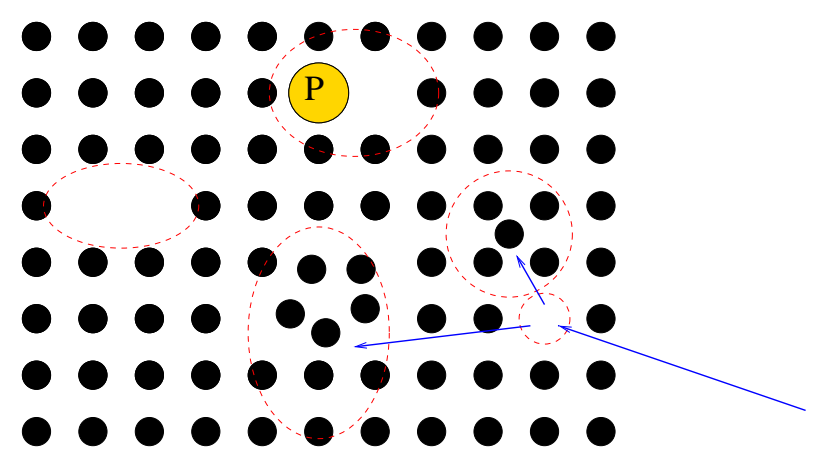

Figure 2.5: Bulk n-type damage. Divacancy, disordered region, interstitial atom and E-center. 
Parametrization of Space Charge Change. The effective doping concentration in the space charge will be the result of an independent occurrence of these processes:

$$
N_{\text {eff }}=N_{\text {donor removal }}+N_{\text {acceptor removal }}+N_{\text {donor creation }}+N_{\text {acceptor creation }}
$$

Modelling every member with the observed fluence dependence (Fig. 2.4) we write Eq. 2.27, with $N_{D, 0}, N_{A, 0}$ donor and acceptor concentration before irradiation and $c_{D}, c_{A}, b_{D}, b_{A}$ constants to be determined experimentally ${ }^{6}$. Assuming an absence of acceptor removal and donor creation, the parametrization for effective doping simplifies to 2.29, as exposed:

$$
\begin{gathered}
N_{e f f}(\Phi)=N_{D, 0} e^{-c_{D} \Phi}-N_{A, 0} e^{-c_{A} \Phi}+b_{D} \Phi-b_{A} \Phi \\
N_{e f f}(\Phi)=N_{D, 0} e^{-c_{D} \Phi}-N_{A, 0} \quad+0 \quad-b_{A} \Phi \\
\Rightarrow \quad N_{e f f}(\Phi)=N_{D, 0} e^{-c_{D} \Phi}-N_{A, 0}-b \Phi .
\end{gathered}
$$

It is worth mentioning at this point that the original detector material contains acceptors and donors simultaneously and only the difference is usually known to some degree of precision. Furthermore, a variety of partially unknown defects will act as effective donors and acceptors. These defect complexes will not be completely stable and will partially selfanneal with different time constants. The vacancy-boron complex, for example, is unstable at room temperature. A simple measurement of the detector depletion voltage can only provide information on the difference of effective donors and acceptors, but not on the separate contributions.

\subsubsection{Reverse-Bias Current}

In the space-charge region a crystal defect will assume predominately a single charge state unless a defect energy level is very close (within a few times $k T$ ) to the intrinsic level. Nevertheless, the defect can change for short times the charge state by emission of electrons and holes -we assume a small leakage current so that capture processes can be ignored. The alternative emission of electron and holes is responsible for volume-generated reverse-bias currents. As the emission probability is exponentially dependent on the position of the defect level in the band gap (see App. F), and emission of both electrons and holes are required, defects with energy levels close to the band-gap center will be most effective in generating leakage currents. As expected from a defect generation proportional to the fluence, a linear relationship between current and fluence is found:

$$
\frac{\Delta I_{v o l}}{V}=\alpha \Phi
$$

${ }^{6} c=3.54 \times 10^{-13} \mathrm{~cm}^{2} \pm 4.5 \%$ and $b=7.94 \times 10^{-2} \mathrm{~cm}^{-1} \pm 8.0 \%([93])$ 
This linearity is seen for electrons and also for neutrons at fluences below type inversion. For fluences above inversion, a stronger rise is observed [25]. See for example Figure 2.6. There the volume-generated current can be parametrized ${ }^{7}$ as:

$$
\frac{\Delta I_{v o l}}{V}=\alpha \Phi+\alpha^{*}\left(\Phi-\Phi_{\text {conv }}\right)
$$

It is interesting to note that after approximately one week of room temperature annealing the damage constants both before and after type inversion converge to the same value of $\alpha=4 \times 10^{-17} \mathrm{Acm}^{-1}$. The damage constant depends not only on the type and energy of irradiation but also shows a strong trivial temperature dependence due to the variation of the instrinsec charge density $n_{i}$ with temperature. We can factor this temperature dependence by expressing the volume-generated current by the generation lifetime $\tau_{g}$ (Ref. [71]) and parametrizing the change of $\tau_{g}$ as:

$$
\frac{1}{\tau_{g}}=\frac{1}{\tau_{g, 0}}+k_{\tau} \Phi
$$

where $\tau_{g, 0}$ is the generation lifetime before irradiation. The two damage constants are related to each other as

$$
\alpha=q n_{i} k_{\tau}
$$

After irradiation, defects are not completely stable. Part of the damage disappears with time even at room temperature. This effect of annealing makes the damage constant timedependent. For comparison of different measurements, it is therefore advisable to quote damage constants right after short irradiations and to treat annealing separately.

The reverse bias current is strongly dependent on temperature. Even after rather low fluences the generation current dominates (appendix $\mathbf{C}$ ), and the reverse bias current has a dependency

$$
I_{R}(T) \propto T^{2} e^{-E / 2 k T}
$$

Unirradiated samples usually exhibit an energy of activation of $E=1.12 \mathrm{eV}$, the gap energy, while for irradiated samples $E=1.2 \mathrm{eV}$. The ratio of reverse bias currents (appendix C) at two temperatures $T_{1}$ and $T_{2}$ is

$$
\frac{I_{R}\left(T_{2}\right)}{I_{R}\left(T_{1}\right)}=\left(\frac{T_{2}}{T_{1}}\right) \exp \left[-\frac{E}{2 k}\left(\frac{T_{1}-T_{2}}{T_{1} T_{2}}\right)\right]
$$

In practice, the variation of leakage current with temperature is very reproducible from device to device, even after substantial doping changes due to radiation damage. The leakage current can be used for dosimetry and diodes are offered commercially specifically for this purpose.

\footnotetext{
${ }^{7}$ With numerical values ([93]) for $n$-type silicon at $20^{\circ} \mathrm{C}$ of: $\alpha=8.0 \times 10^{-17} \mathrm{Acm}^{-1}, \alpha^{*}=9.8 \times 10^{-17} \mathrm{~cm}^{-1}$, $\Phi_{c o n v}=4 \times 10^{12} \mathrm{~cm}^{-2}$
} 


\subsection{Annealing of Radiation Damage}

\subsubsection{Beneficial Annealing}

Considering a radiation-damaged detector after the end of the irradiation process, it is observed that the damage to the detector (i.e. effective dopping change and increase of leakage current) diminuishes with time. The rate of damage decrease is strongly dependent on the temperature at which the detector is kept during the observation (no irradiation) period. As this observation can be interpreted as a partial disappearance of radiation-generated crystal defects, the effect has been called "annealing". Defects and defect complexes are in general stable only up to a characteristic temperature $T_{a n n}$, the "annealing temperature" (Table 2.3). An exponential behavior of the annealing is assumed of the form:

$$
N_{d}(t)=N_{d}(0) e^{-\frac{t}{\tau}} \quad \text { with } \quad \tau(T) \propto e^{\frac{E_{a}}{k T}}
$$

where $E_{a}$ is the activation energy (values displayed in Table 2.3) and the order of magnitude of $\tau\left(T_{a n n}\right)$ is $\mathbf{1 0}$ minutes. The annealing phenomenon is a complicated process involving many different and only partially understood processes between defects and defects complexes. As new defect complexes are produced, the effect of annealing may not always be beneficial for detector performance. An example of such a detrimental effect is the increase of the effective doping concentration in the space charge after initial annealing of intensely irradiated detectors, an effect called "reverse annealing".

Parametrization of Beneficial Annealing. Since a quantitative description of the radiation damage with a physical model is not possible, the observed effects have to be parametrized. This is done separately for the effective doping, the volume-generated current, and the trapping. The effective doping is just a simple way to describe the space-charge density in a fully depleted semiconductor region including the (partially) ionized defects. In all cases one assumes that there exist several defect types that decay with their characteristic times. The rate of introduction of radiation-induced volume-generated current has been found to be roughly a factor two higher after type inversion than for low irradiation fluences (eqs. 2.30 and 2.31, where $\alpha \sim \alpha^{*}$ ). The annealing behavior shown (from experimental data see [71], Figs. 11.15, 11.16) is also drastically different, so that after a period of roughly one week both show similar remaining damage. Parametrizing this behavior, we write for the effective doping concentration $N_{e f f}$ and for the volume-generated (leakage) current:

$$
\frac{\Delta I(t)}{\Delta I(0)}=\sum_{i=1}^{n} A_{i} e^{-\frac{t}{\tau_{i}}}
$$

\footnotetext{
${ }^{8}$ The annealing temperature is not accurately defined as even below this temperature some annealing occurs.
} 


$$
\frac{\Delta N_{e f f}(t)}{\Delta N_{e f f}(0)}=\sum_{i=1}^{n} A_{i} e^{-\frac{t}{\tau_{i}}}
$$

\subsubsection{Reverse Annealing.}

When performing systematic studies of radiation damage of silicon detectors, a surprising new effect was found. Measuring the effective doping of irradiated silicon as afunction of time, it was observed that the initial decrease of doping change during the first few weeks of room temperature annealing was followed by a new increase of the effective doping change, a process proceeding on the time scale of many months ([93]). As the effective doping change increases, this efect was called "reverse annealing".

Parametrization of Reverse Annealing. A natural explanation for such behavior is the transformation of radiation-induced electrically inactive defect complexes into electrically active defects. Two different mechanisms have been proposed:

$\star$ A first-order process where the slow decay of inactive complexes of type $\mathbf{X}$ into electrically active type $Y$ defects;

$\star$ A second-order process where a reaction between two kinds of inactive defects $X_{1}$ and $X_{2}$ creates an electrically active defect complex $Y$.

For the first-order process the decay rate is proportional to the concentration:

$$
\begin{gathered}
\frac{d N_{Y}}{d t}=-\frac{d N_{X}}{d t}=\hat{k}(T) N_{X} \\
\Rightarrow \quad N_{Y}(t, T)=N_{X_{0}}\left(1-e^{-t / \tau}\right) \quad \text { with } \quad \tau=1 / \hat{k}(T) .
\end{gathered}
$$

For the second-order effect the interaction rate is proportional to the product of defect concentrations:

$$
\frac{d N_{Y}}{d t}=-\frac{d N_{X_{1}}}{d t}=-\frac{d N_{X_{2}}}{d t}=\tilde{k}(T) N_{X_{1}} N_{X_{2}}
$$

Solving the system and assuming nearly equal concentrations $N_{X_{0}}(\Phi)$ of the two types of radiation-induced primary defects [94], the new type of defect being responsible for the increase in effective doping has a time dependence of the form

$$
N_{Y}(\Phi, t, T)=N_{X_{0}}(\Phi)\left(1-\frac{1}{1+N_{X_{0}}(\Phi) \tilde{k}(T) t}\right) .
$$


And both models have the same saturation value when time $t$ tends to infinity: $N_{Y_{\infty}}=N_{X_{0}}$. However, for the second-order process the rise time is expected to depend on the defectconcentration, thus resulting in a different form of the time dependence compared to a first order process.

Reverse annealing can be accelerated by raising the temperature and slowed down by cooling. Elevated temperatures are used for investigating reverse annealing in a reasonable time scale. Cooling is applied to reduce or completely suppress (below approximately $0^{\circ} \mathrm{C}$ ) reverse annealing in the operation of detectors in high radiation environments.

\subsection{Parametrization of Radiation Damage.}

In the previous sections we have parametrized the change that radiation damage creates to the effective doping concentration of the sensor's space charge. The three different contributions were:

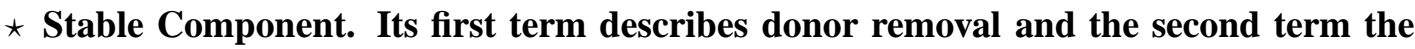
linear formation of acceptors. This expression is called stable because it does not depend on time or temperature, only fluence:

$$
\Delta N_{C}(\Phi)=N_{C 0}\left(1-e^{-c \Phi}\right)+g_{C} \Phi
$$

where $g_{C}$ is the introduction rate of stable acceptors.

$\star$ Beneficial Annealing. Term that describes a recovery from the change in space charge as an exponential decay of acceptors ${ }^{9}$ :

$$
\Delta N_{A}(\Phi, t, T)=\Phi \sum_{i} g_{a_{i}} e^{-t / \tau_{a_{i}}(T)}
$$

where $g_{a_{i}}$ are the introduction rates and $\tau_{a_{i}}$ the time constants (which can be approximated by one single decay with average values $g_{a}$ and $\tau_{a}$ ).

* Reverse Annealing. A long-term effect that increases the number of acceptor-like sites $^{10}$ :

$$
\Delta N_{Y}(\Phi, t, T)=g_{Y} \Phi\left(1-\frac{1}{1+t / \tau_{Y}(T)}\right)
$$

where $g_{Y}$ is the introduction rate and $\tau_{Y}$ the time constant.

\footnotetext{
${ }^{9}$ Beneficial annaeling has a time constant of $55 \mathrm{~h}$ at $20^{\circ} \mathrm{C}$, or $19 \mathrm{~min}$ at $60^{\circ} \mathrm{C} \quad$ [23].

${ }^{10}$ Reverse Annealing has a time constant of 475 days at $20^{\circ} \mathrm{C}$ or $1260 \mathrm{~min}$ at $60^{\circ} \mathrm{C}$ [23].
} 
As a cumulative effect of these processes, the change in space charge is ${ }^{11}$ [77]

$$
\Delta N_{e f f}(\Phi, t, T)=N_{e f f, 0}-N_{e f f}(\Phi, t, T)=\Delta N_{C}(\Phi)+\Delta N_{A}(\Phi, t, T)+\Delta N_{Y}(\Phi, t, T)
$$

where a positive or negative sign of $N_{e f f}$ (effective doping concentration of $n$-type starting material) denotes whether the effective doping is $n$ - or $p$ - like [70].

Figure 2.7 shows the evolution of the beneficial annealing and reverse annealing $v s$. time at a temperature $20^{\circ} \mathrm{C}$ and $-5^{\circ} \mathrm{C}$ after a fluence burst of $10^{14} \mathrm{~cm}^{-2}$

At room temperature beneficial annealing reduces the change in space charge at times $<10^{6} \mathrm{~s}(\sim 12 \mathrm{~d})$. Then anti-annealing dominates, increasing $N_{\text {eff }}$ to about $6 \times 10^{12} \mathrm{~cm}^{-3}$ over the course of a year. If the starting donor concentration is $1 \times 10^{12} \mathrm{~cm}^{-3}$ this requires a bias voltage of $360 \mathrm{~V}$. Operating the detector at $-5^{\circ} \mathrm{C}$ delays anti-annealing. In fact, the increase in fluence and the annealing proceed concurrently, so Fig. 2.7 does not apply directly, but it illustrates that operating the detector at low temperature and only allowing warm-up during annual maintenance periods is critical. This low temperature will reduce the leakage current (reverse bias current) but it should not be too low to suppress the desiderable effects of beneficial annealing. Operation can be extended by utilizing detector configurations that do not require that charge drift over the whole detector thickness. The induced signal in highly segmented detectors peaks near the electrode. Since charge motion near the opposite electrode does not contribute much to the total integrated charge signal, a useful signal can still be obtained for carriers that only partially traverse the detector. In LHC pixel systems this is obtained by implementing the detector with $n^{+}$electrodes in an $n$-substrate. After inversion this behaves like $n^{+}$electrodes in an $p$-substrate. When operated at less than the "depletion" voltage electrons are still collected at the $n$-electrodes, but not from the full detector thickness, so the signal is reduced. The detector ceases to be usable when the signal-to-noise ratio becomes too small (but the limits can be extended). Small electrode areas reduce both the reverse bias current and the capacitance, so reduced noise extends the operation of pixel detectors with respect to strip devices ${ }^{12}$.

\subsection{Mitigation Techniques}

Many techniques can be applied to reduce the effects of radiation damage to an overall system. The goal of radiation-hard design is not so much to obtain a system whose characteristics do not change under irradiation, rather than to maintain the required performance characteristics over the lifetime of the system. The former approach tends to utilize

\footnotetext{
${ }^{11}$ Expression of the so-called Hamburg Model.

${ }^{12}$ The ATLAS pixel system has noise levels $\sim 200 e$ whereas the strip systems operate at $\sim 1500 e$.
} 
mediocre to poor technologies that remain so over the course of operation. The latter starts out with superior characteristics, which gradually deteriorate under irradiation. Depending on the specific system, these designs may die gradually, although at some fluence or dose a specific circuit, typically digital, may cease to function at all.

Increased detector leakage current has several undesirable consequences:

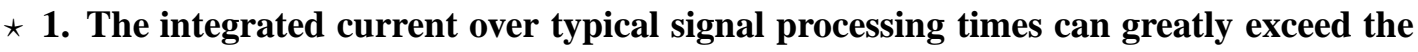
signal.

$\star 2$. Increase of shot noise.

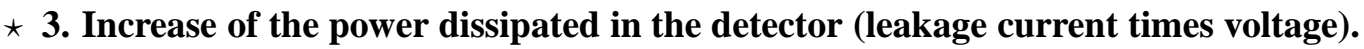

Since the leakage current decreases exponentially with temperature, cooling is the simplest technique to reduce diode leakage current. For example, reducing the detector temperature from room temperature to $0 \mathrm{C}$ reduces the leakage current to about $1 / 6$ of its original value.

Detector power dissipation is a concern in large-area silicon detectors (e.g. CMS at LHC) where the power dissipation in the detector diode itself can be of order $1-10 \mathrm{~mW} / \mathrm{cm}^{2}$. Since the leakage current is an exponential function of temperature, local heating will increase the leakage current, which will increase the local heating, and so on, ultimately taking the device into thermal runaway. To avoid this potentially catastrophic failure mode, the cooling system must be designed to provide sufficient cooling of the detector.

Reducing the integration time reduces both baseline changes due to integrated detector current and shot noise. Clearly, this is limited by the duration of the signal to be measured. To some degree, circuitry can be designed to accomodate large baseline shifts due to detector current, but at the expense of power. AC coupled detectors eliminate this problem. An instrumentation systems that require DC coupling, correlated double sampling techniques can be used to sample the baseline before the signal occurs and then subtract from the signal measurement.

One of the most powerful measures against detector leakage current is segmentation. For a given damage level, the detector leakage current per signal channel can be reduced by segmentation. If a diode with a leakage current of $10 \mu \mathrm{A}$ is subdivided into 100 subelectrodes each with its own signal processing channel, the leakage current in each channel will be $100 \mathrm{nA}$ and shot noise reduced by a factor of 10. This is why large area silicon tracking detectors can survive in a hard radiation environment (e.g. LHC). Fortuitously, increased segmentation is also required to deal with the high event rate. Pixel detectors with small electrode areas offer great advantages in this regard. 
The most severe restriction on radiation resistance is imposed by type inversion in the sensor, when the net space charge becomes so large that the detector will no longer sustain the required voltage for full charge collection. This is especially critical for position-sensing detectors with electrodes on both sides (double-sided detectors), for which full collection is essential.

Type-inversion limit can be eluded by using back-to-back single-sided detectors. The initial configuration uses $n$-type segmented strip electrodes on $n$ bulk, with a contiguous $p$ electrode on the backside. Initially, the $p n$-junction is at the backside. This does require full depletion in initial operation; this is not a problem for the nonirradiated device and becomes easier to maintain as increasing fluence moves the bulk towards type inversion. After type-inversion the charge collection region extends from the $n$ electrodes. Since most of the signal charge is induced when the carriers are near the strip or pixel electrodes, this provides good efficiency even when operating at less than the nominal depletion voltage.

Since highly damaged detectors are largely devoid of mobile charge, they appear approximately ohmic. This means that reverse bias is not essential to obtain low acceptable leakage currents and the detectors also function under forward bias. Chilingarov and Sloan ([95]) demonstrated good charge collection efficiency with forward bias voltages much smaller than the reverse bias required for the same signal. Although the bias current is larger than for reverse bias, this results in less dissipation in the detector and also simplifies the detector design. However, the smaller fields also lead to longer collection times, so the effect of trapping is exacerbated. To some degree this is ameliorated by the fact that electron traps are more readily filled by the larger standing current.

A innovative approach to avoiding large operating voltages while reducing collection times is the "3D detector". Rather than forming the diode between opposite faces of a wafer, the electrodes are alternating columns of $n^{+}$and $p^{+}$material that are normal to the surface of the $p$ bulk. Columns have been implemented with $300 \mu \mathrm{m}$ depth and a diameter about $5 \%$ of the depth. In one set of test devices the pitch within a row is $200 \mu \mathrm{m}$ and adjacent $n$ - and $p$ - rows are offset by $100 \mu \mathrm{m}$ so that the distance that must be depleted is $\approx 140 \mu \mathrm{m}$. By appropriate choice of geometry the distance between $n$ - and $p$-columns can be much smaller than the wafer thickness, so that both the required voltage for full collection and the collection times are reduced. For example a spacing of $50 \mu \mathrm{m}$ between $n$ - and $p$-columns would yield full charge collection at order of magnitude smaller voltage than conventional devices. Devices have been irradiated to fluences $>5 \cdot 10^{14} \mathrm{~cm}^{-2}$ of $24 \mathrm{GeV} / \mathrm{c}$ pions with good results. The same technology can be applied as trenches at the edges of the detector to provide "active" edges to avoid the dead area at the edges of conventional devices. This is very useful in tiling detectors to form large area arrays for tracking or x-ray imaging. A similar principle has been applied to planar devices by placing alternating $n^{+}$and $p^{+}$ 
electrodes on opposite faces of a double-sided detector.

Another path that is being pursued is operation at cryogenic temperatures. Since the build-up of space charge results from the population of acceptor-like states through thermal excitation, heavily damaged detectors can be resuscitated by cooling to liquid nitrogen temperature $^{13}$. Charge trapping is not suppressed, so roughly half of the charge is recovered when operating a $300 \mu \mathrm{m}$ thick detector at $250 \mathrm{~V}$ after irradiation to a neutron fluence of

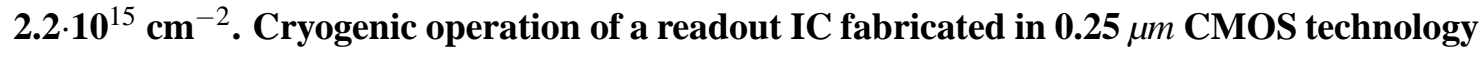
has also been demonstrated. Silicon detectors operating at $130 \mathrm{~K}$ are in use as high-intensity radiation monitors.

Finally, another way to avoid the limits of silicon is to use different materials. In partricaulr, Diamond has been shown to be quite radiation resistant, with a $15 \%$ degradation in signal-to-noise after exposure to a $24 \mathrm{GeV}$ proton fluence of $2.2 \cdot 10^{15} \mathrm{~cm}^{-2}$. Diamond is an insulator, so bias currents are very low without resorting to $p n$-junctions and their inherent doping effects. However, the large bandgap also reduces the charge yield. Minimum ionizing particles on average produce 36 electron-hole pairs per micron, about half the charge obtained with silicon. This is partially mitigated by a smaller dielectric constant, relative to silicon ( $\varepsilon \approx 5.5$ vs. 12.9). Apart from cost, a major limitation is the obtainable drift length, which depends greatly on the growth techniques. In polycrystalline material drift lengths of $\sim 200 \mu \mathrm{m}$ have been achieved. Diamond pixel sensors have been operated successfully with readout ICs designed for ATLAS silicon pixel system. Single crystal synthetic diamond is now available and has been applied in beam monitoring.

\subsection{Semiconductors in Particle Physics}

Silicon and other semiconductor detectors were used extensively in nuclear physics experiments long before the widespread development of semiconductor devices in electronics. During the late 50s and early 60s, semiconductor detectors found various applications in nuclear physics. While they could not be used for large area coverage as could scintillating materials, their advantages in some specific applications prompted their development.

Since the observation by McKay [26] in 1951 that $\alpha$ particles produced a measurable signal when they impinged on a reverse-biased n-p junction in germanium, all semiconductor detectors have been based on the collection of charge carriers from a depletion junction. Such structure combines some convenient properties of insulators (no free charges carriers and therefore no current in the presence of the imposed electric field) and some from conductors (rapid transport out of the depletion region of any charge carriers generated, giving

\footnotetext{
${ }^{13}$ Lazarus effect
} 
a current which can be detected with external electronics).

The energy required for creation of an electron-hole pair in silicon is about $3.6 \mathrm{eV}$, in contrast to about $30 \mathrm{eV}$ in a gaseous detector and $300 \mathrm{eV}$ for a scintillator to generate one photoelectron (at the photocatode of a photomultiplier tube). Combined with its high stopping power, this allows the construction of silicon detectors of excellent energy resolution (e.g. a $10 \mathrm{MeV}$ proton stops in $1 \mathrm{~mm}$ of silicon, releasing about $2.8 \times 10^{6}$ electron-hole pairs, yielding an intrinsic energy resolution of $\left.6 \times 10^{-4}[30]\right)$.

In high energy physics, the discovery of the charmed [27] and beauty [28, 29] particles and the fact that they proved to be relatively long-lived $\left(>10^{-13} \mathrm{~s}\right.$, so that they may travel $\sim 1 \mathrm{~mm}$ from their production point in a high energy collision before they decay) provided a major stimulus for the developement of vertex and tracking detectors with high spatial precision $(<\mathbf{1 0} \mu \mathrm{m})$.

\subsubsection{Silicon Particle Detectors}

Most silicon particle detectors work by doping narrow (usually around $\mathbf{1 0 0}$ micrometers wide) strips of silicon to make them into diodes, which are then reverse biased. As charged particles pass through these strips, they cause small ionization currents which can be detected and measured. Arranging thousands of these detectors around the collision point of a particle accelerator can give an accurate picture of the particles' path. By drawing each path back to where it meets with one or more other paths, it is possible to find the position where any given charged particle was created: the vertex (since charged particles are never created alone but always in pairs of equal and opposite charges). A vertex created outside the collision region indicates that a very short-lived particle was formed in the collision and then decayed at the vertex location to produce the particles whose tracks are seen emerging from the vertex itself. In this way it is possible to detect the production of unstable particles containing massive charm and bottom (b) quarks (b-tagging). Silicon detectors have a much higher resolution in tracking charged particles than older technologies such as cloud chambers or wire chambers. The drawback is that silicon detectors are much more expensive than these older technologies and require sophisticated cooling to reduce leakage currents (noise source) as well as suffer degradation over time from radiation.

In a silicon particle detector, radiation is measured by means of the number of charge carriers set free in the detector, which is arranged between two electrodes. Ionizing radiation produces free electrons and holes when traversing the bulk material of the sensor and the number of electron-hole pairs is proportional to the energy transmitted by the radiation to the semiconductor. As a result, a number of electrons are transferred from the valence band to the conduction band, and an equal number of holes are created in the valence band. 
Under the influence of an electric field, electrons and holes travel to the electrodes, where they result in a pulse that can be measured in an outer circuit. The holes travel into the opposite direction and can also be measured. As the amount of energy required to create an electron-hole pair is known, and is independent of the energy of the incident radiation, measuring the number of electron-hole pairs allows the energy of the incident radiation to be found.

\subsubsection{Vertex and Tracking Detectors at Hadron Colliders}

Experiments at hadron colliders must deal with high interaction rates. However, most of the interactions are background so pattern recognition is crucial, which increases the number of layers required for efficient track reconstruction. Furthermore, the interaction region tends to be spread in length (about $50 \mathrm{~cm}$ in Tevatron) so the detectors must be designed suitably long.

\subsubsection{CDF and DO at the Tevatron}

CDF at the Fermi National Laboratory installed a silicon vertex detector early in its operation in 1987 and since then has operated a succesion of upgrades detectors. The original SVX had four concentric barrel layers read out at both ends, for a total channel count of 37000 . Since only a small fraction of the strips would be struck in a given event, SVX pioneered onchip sparsification which selects only struck channels for readout. With this technology the required readout bandwidth is independent of segmentation, a crucial consideration in the very large detectors already envisaged for the next generation of high luminosity colliders. For the upgraded Tevatron CDF substantially expanded coverage of the vertex detector and added silicon layers at radii beyond $10 \mathrm{~cm}$ to enhance particle tracking. A "Layer 00" was also mounted at the smallest possible radius just outside the beam pipe.

As coverage is extended to forward angles, a barrel geometry requires inordinate silicon area and resolution suffers for grazing incidence tracks. A novel layout used at Tevatron by DO was implemented, consisting on interspersed barrels and disks in the interaction region and additional disks to provide forward coverage to $\eta^{14} \approx 3$.

\subsubsection{ATLAS and CMS at the LHC}

The LHC poses unprecedented challenges to detector design. Work on suitable detector concepts began in th 1980s, culminating in final assembly in 2005-2007. A worldwide R\&D

\footnotetext{
${ }^{14}$ The $\eta$ coordinate (pseudo-rapidity) is a transformation of the polar angle $\theta$ under the formula $\eta=-\ln (\tan (\theta / 2)$.
} 
program was neccessary to develop the concepts and technologies, especially in the areas of sensors, microelectronics and radiation effects. A general purpose detector includes vertexing for b-tagging, precision tracking in a magnetic field (2 $\mathrm{T}$ in ATLAS, $4 \mathrm{~T}$ in CMS), calorimetry and muon detection. ATLAS and CMS use barrels in the central region and disks in the forward regions to provide the required coverage and tracking performance with minimum silicon area. The ATLAS SemiConductor Tracker (SCT) has about $60 \mathrm{~m}^{2}$ of silicon with $60 \times 10^{6}$ strip detector channels, augmented by a gaseous outer tracking detector. CMS has an all-silicon tracker with about $230 \mathrm{~m}^{2}$ of silicon and $10^{7}$ strip detector channels. Both ATLAS and CMS use CMOS pixel devices covering 1-2 $\mathrm{m}^{2}$ with 50-100 million channels at the inner radii $(<15 \mathrm{~cm})$ because of their superior pattern recognition at high track densities and radiation resistance. Microstrip technology takes over at larger radii to minimize material and cost.

In ATLAS the pixels are $50 \mu \mathrm{m} \times 400 \mu \mathrm{m}$, with the long dimension along the beam axis to accomodate inclined tracks. Fluence after 10 years of operation is estimated to be $10^{15} \mathrm{~cm}^{-2}$ (1MeV neutron equivalent) with a total dose of $50 \mathrm{Mrad}$ at the innermost pixel layer and a fluence of $2 \cdot 10^{14} \mathrm{~cm}^{-2}$ at the inner strip layer. The strip silicon orientation is $<111>$, because of easier availability.

CMS uses an all-silicon tracker with $2.4 \mathrm{~m}$ diameter and $5.4 \mathrm{~m}$ length in a $4 \mathrm{~T}$ solenoidal magnetic field. Strip detectors are used in all layers except at the smallest radii, where the interaction region is surrounded by two barrel layers of pixel detectors of size $100 \mu \mathrm{m} \times$ $150 \mu \mathrm{m}$. In the strip detector portion double-sided detectors are used in layers 1, 2, 5, and 6 of the barrel and in rings 1, 2, and 5 of the disks. As in ATLAS the double-sided modules use two single-sided sensors, glued back-to-back to form a small stereo angle. Sensors use the $<\mathbf{1 0 0}>$ orientation to minimize surface damage. 


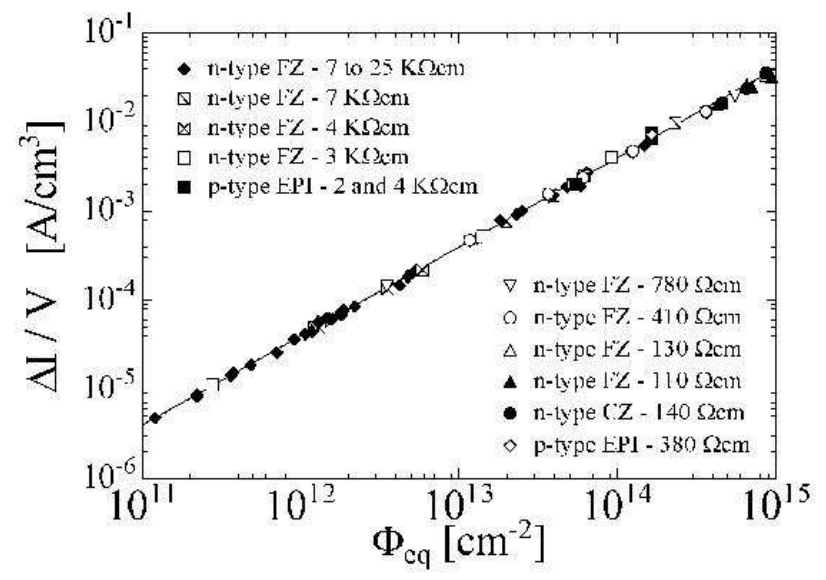

Figure 2.6: Fluence dependence of leakage current for silicon detectors produced by various process technology from different silicon materials. The current was treated after a heat treatment for $80 \mathrm{~min}$ at $60^{\circ} \mathrm{C}$ (see Ref. [25]). 

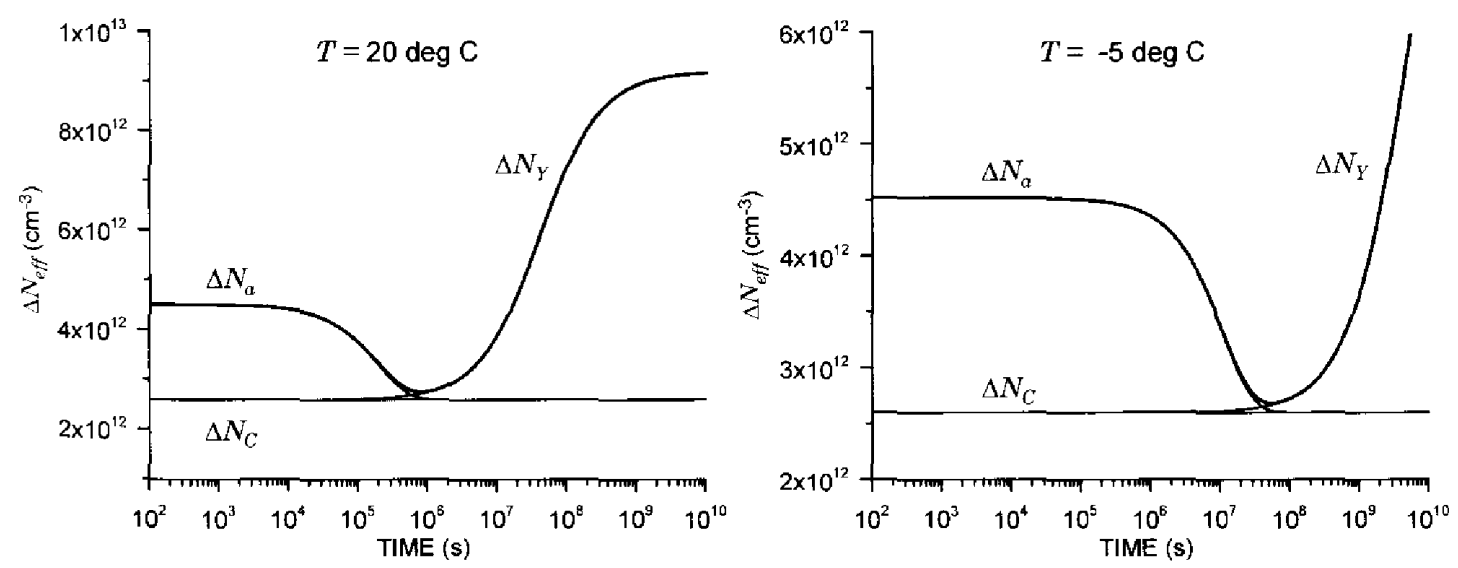

Figure 2.7: Evolution of beneficial annealing $\Delta N_{a}$ and anti-annealing $\Delta N_{Y} v s$. time at $20^{\circ} \mathrm{C}$ and $-5{ }^{\circ} \mathrm{C}$ after proton fluence burst of $10^{14} \mathrm{~cm}^{-2}$. 



\section{Chapter 3}

\section{CDF at Fermilab}

The data for the studies described in this thesis was taken with the Collider Detector at Fermilab (CDF) located at the Fermi National Accelerator Laboratory. In the following sections, a brief introduction to the Tevatron Collider and a description of the CDF detector are given, with a particular attention to the detector components that have been more relevant for the studies.

\subsection{The Tevatron Collider}

The Tevatron Collider [1] located at the Fermi National Accelerator Laboratory (Fermilab) in Batavia (Illinois, USA) is a proton-antiproton $(p \bar{p})$ collider with a center-of-mass energy of 1.96 TeV. As shown in figure 3.1, this complex has five major accelerators and storage rings used in successive steps, as is explained in detail below, to produce, store and accelerate the particles up to $980 \mathrm{GeV}$.

The acceleration cycle starts with the production of protons from ionized hydrogen atoms $H^{-}$, which are accelerated to $750 \mathrm{KeV}$ by a Cockroft-Walton electrostatic accelerator. Preaccelerated hydrogen ions are then injected into the Linac where they are accelerated up to $400 \mathrm{MeV}$ by passing through a $150 \mathrm{~m}$ long chain of radio-frequency (RF) accelerator cavities. To obtain protons, the $H^{-}$ions are passed through a carbon foil which strips their electrons off. Inside the Booster the protons are merged into bunches and accelerated up to an energy of $8 \mathrm{GeV}$ prior to entering the Main Injector. In the Main Injector, a synchrotron with a circumference of $3 \mathrm{~km}$, the proton bunches are accelerated further to an energy of $150 \mathrm{GeV}$ and coalesced ${ }^{1}$ together before injection into the Tevatron.

\footnotetext{
${ }^{1}$ coalescing is the process of merging proton bunches into one dense, high density, bunch
} 
The production of the antiproton beam is significantly more complicated. The cycle starts with extracting a $120 \mathrm{GeV}$ proton beam from the Main Injector onto a stainless steel target. This process produces a variety of different particles, among which are antiprotons ${ }^{2}$. The particles come off the target at many different angles and they are focused into a beam line with a Lithium lens. In order to select only the antiprotons, the beam is sent through a pulsed magnet which acts as a charge-mass spectrometer. The produced antiprotons are then injected into the Debuncher, an $8 \mathrm{GeV}$ synchrotron, which reduces the spread in the energy distribution of the antiprotons. After that, the antiproton beam is directed into the Accumulator, a storage ring in the Antiproton Source, where the antiprotons are stored at an energy of $8 \mathrm{GeV}$ and stacked to $10^{12}$ particles per bunch. The antiproton bunches are then injected into the Main Injector and accelerated to $150 \mathrm{GeV}$.

\section{FERMILAB'S ACCELERATOR CHAIN}

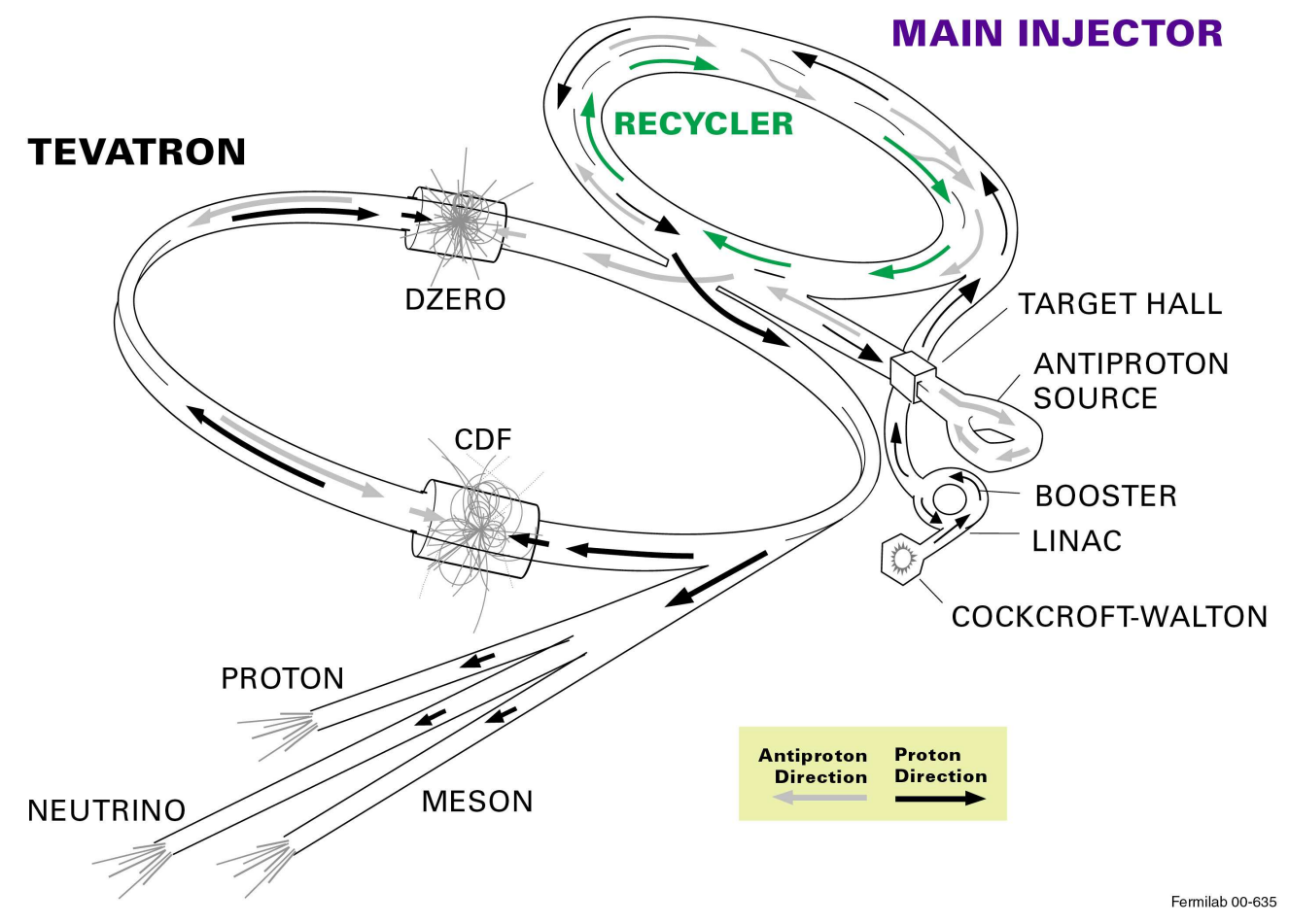

Figure 3.1: The Tevatron Collider Chain at Fermilab.

Finally, 36 proton and antiproton bunches are inserted into the Tevatron, a double acceleration ring of $1 \mathrm{~km}$ of radius, where their energy is increased up to $980 \mathrm{GeV}$. Proton and antiproton bunches circulate around the Tevatron in opposite directions guided by super-

\footnotetext{
${ }^{2}$ The production rate, for $8 \mathrm{GeV}$ antiprotons, is about $18 \bar{p} / 10^{6} p$
} 
conducting magnets and where their orbits cross at the two collision points, B0 and D0. These interactions are observed by the CDF and DO detectors, respectively.

In the absence of a crossing angle or position offset, the luminosity at the CDF or DO interaction point is given by the expression:

$$
L=\frac{f_{b c} N_{b} N_{p} N_{\bar{p}}}{2 \pi\left(\sigma_{p}^{2}+\sigma_{\bar{p}}^{2}\right)} F\left(\frac{\sigma_{l}}{\beta^{*}}\right),
$$

where $f_{b c}$ is the revolution frequency, $N_{b}$ is the number of bunches, $N_{p(\bar{p})}$ is the number of protons (antiprotons) per bunch, and $\sigma_{p(\bar{p})}$ is the transverse and longitudinal rms proton (antiproton) beam size at the interaction point. $F$ is a form factor with a complicated dependence on beta function, $\beta^{*}$, and the bunch length, $\sigma_{l}$. The beta function is a measure of the beam width, and it is proportional to the beam's $x$ and $y$ extent in phase space. Table 3.1 shows the design Run II accelerator parameters [2].

\begin{tabular}{|l|c|}
\hline Parameter & Run II \\
\hline number of bunches $\left(N_{b}\right)$ & 36 \\
revolution frequency $[\mathrm{MHz}]\left(f_{b c}\right)$ & 1.7 \\
bunch rms $[\mathrm{m}] \sigma_{l}$ & 0.37 \\
bunch spacing $[\mathrm{ns}]$ & 396 \\
protons/bunch $\left(N_{p}\right)$ & $2.7 \times 10^{11}$ \\
antiprotons/bunch $\left(N_{\bar{p}}\right)$ & $3.0 \times 10^{10}$ \\
total antiprotons & $1.1 \times 10^{12}$ \\
$\beta^{*}[\mathrm{~cm}]$ & 35 \\
\hline
\end{tabular}

Table 3.1: Accelerator parameters for Run II configuration.

Figures 3.2 and 3.3 show, respectively, the evolution in the integrated luminosity, defined as $\mathcal{L}=\int L d t$, and the instantaneous luminosity delivered by Tevatron since the machine was turned on up to February 2006. The progressive increase in the integrated luminosity and the continuous records in the instantaneous luminosity ${ }^{3}$ prove the good performance of the accelerator.

\footnotetext{
${ }^{3}$ At February 2009, the record in the instantaneous luminosity was close to $2.8 \times 10^{32} \mathrm{~cm}^{-2} \mathrm{~s}^{-1}$.
} 
Collider Run II Integrated Luminosity

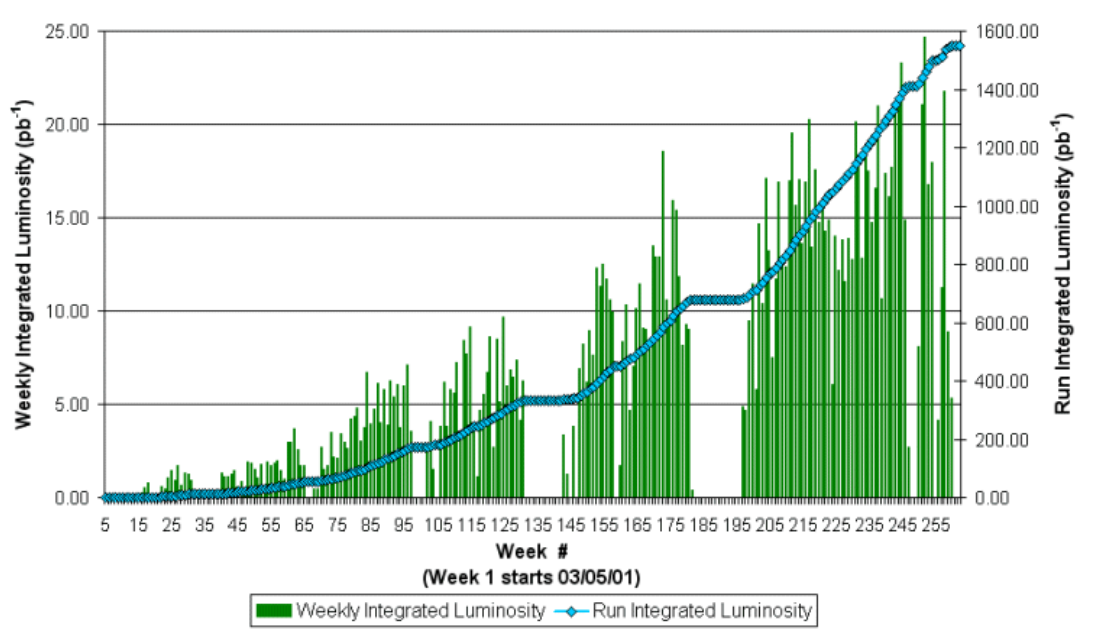

Figure 3.2: Tevatron Collider Run II Integrated Luminosity. The vertical green bar shows each week's total luminosity as measured in $\mathrm{pb}^{-1}$. The diamond connected line displays the integrated luminosity.

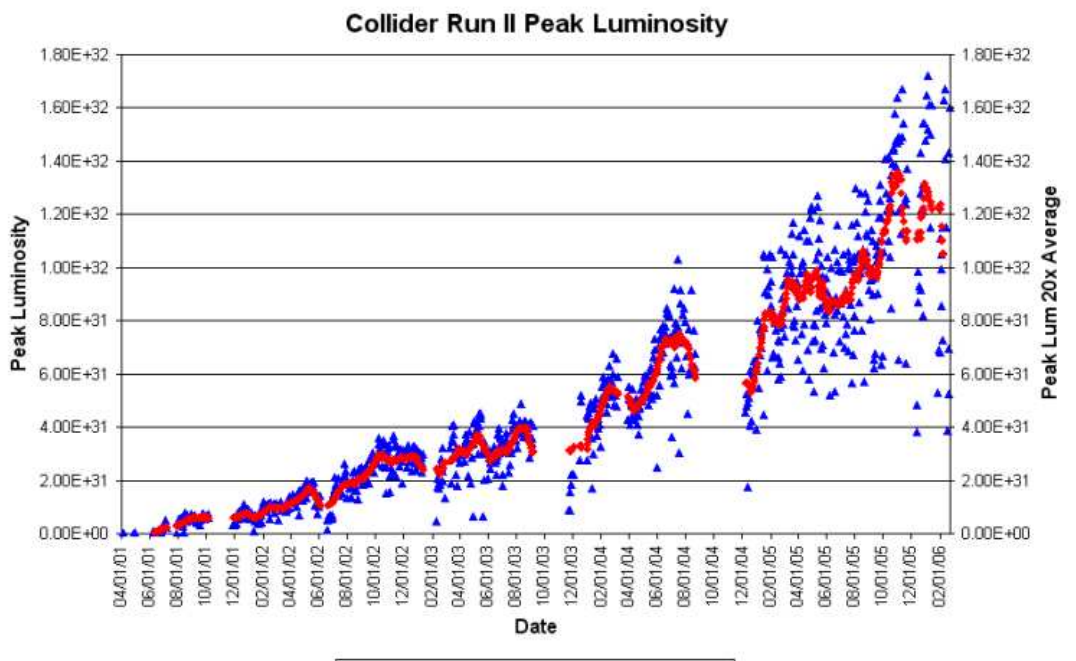

$\triangle$ Peak Luminosity $\bullet$ Peak Lum 20x Average

Figure 3.3: Tevatron Collider Run II Peak Luminosity. The blue squares show the peak luminosity at the beginning of each store and the red triangle displays a point representing the last 20 peak values averaged together. 


\subsection{CDF Run II detector}

The CDF Run II detector [3], in operation since 2001, is an azimuthally and forwardbackward symmetric apparatus designed to study $p \bar{p}$ collisions at the Tevatron. It is a general purpose, cylindrical-shaped detector which combines:

- A tracking system, that provides a measurement of the charged particle momenta, event $\mathrm{z}$ vertex position and detects secondary vertices.

- A Time-of-Flight system, to identify charged particles.

- A non-compensated calorimeter system, with the purpose of measuring the energy of charged and neutral particles produced in the interaction.

- Drift chambers and scintillators to muon detection.

The detector is shown in figures 3.4 and 3.5. CDF uses a coordinate system with the positive $z$-axis lies along the direction of the incident proton beam, $\phi$ is the azimuthal angle, $\theta$ is the polar angle (measured from the detector center), and $p_{T}$ is the component of momentum in the transverse plane. A description of all the systems starting from the devices closest to the beam and moving outward is presented in the next sections, where the detectors most relevant in the analysis are explained in more detail.

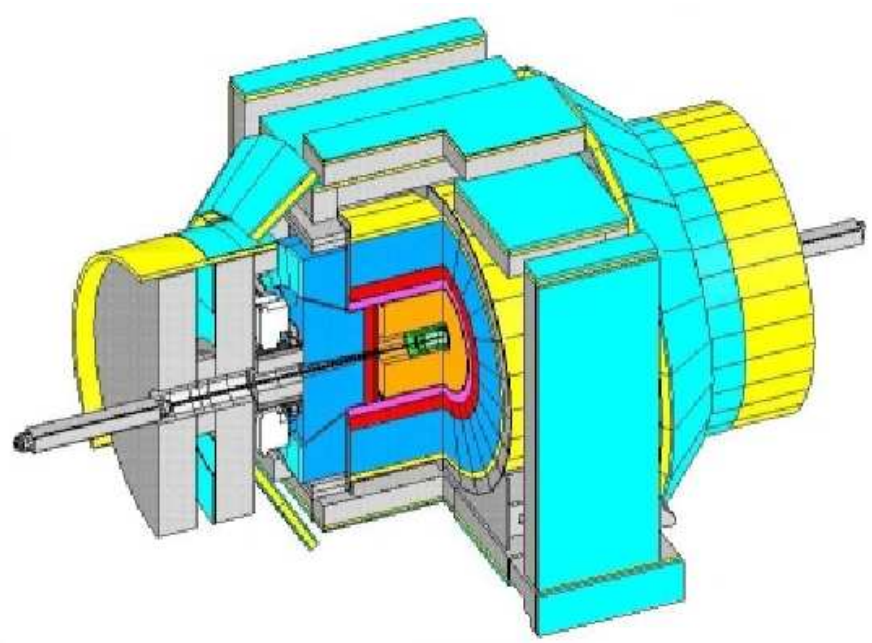

Figure 3.4: Isometric view of the CDF Run II detector. 


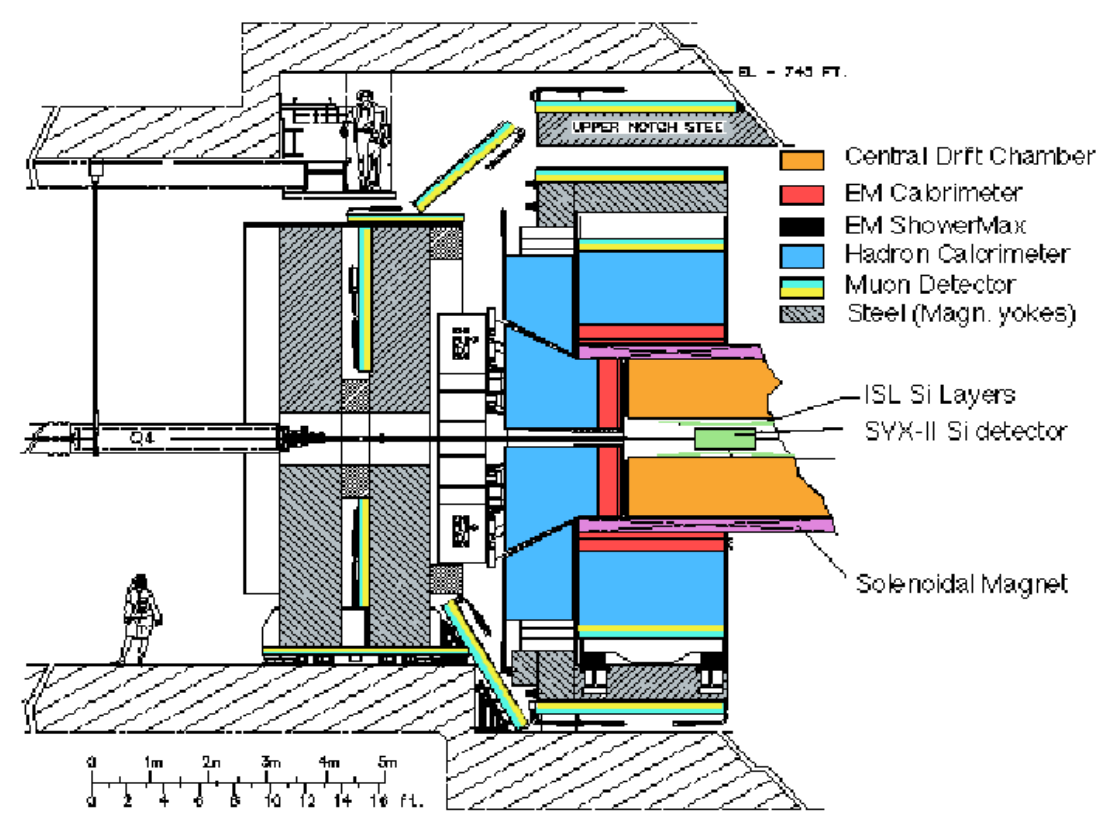

Figure 3.5: $r \times \eta$ side view of the CDF Run II detector.

\subsubsection{Tracking and Time of Flight systems}

The tracking and time of flight systems are contained in a superconducting solenoid, $1.5 \mathrm{~m}$ in radius and $4.8 \mathrm{~m}$ in length, which generates a $1.4 \mathrm{~T}$ magnetic field parallel to the beam axis.

The part of the tracking system closest to the beam pipe is a silicon microstrip detector [4], which must be radiation-hard due its proximity to the beam. It extends from a radius of $r$ $=1.5 \mathrm{~cm}$ from the beam line to $\mathbf{r}=28 \mathrm{~cm}$, covering $|\eta|<2$ and has eight layers in a barrel geometry. The innermost layer is Layer 00 , a single-sided silicon microstrip detector which provides a $r \times \phi$ position measurement. The first five layers after the Layer 00 constitute the Silicon Vertex Detector (SVXII) and the two outer layers comprise the Intermediate Silicon Layers system (ISL). These seven layers are made of double-sided silicon sensors, giving $r \times \phi$ and $z$ position information. The best position resolution achieved is $9 \mu \mathrm{m}$ in SVXII and the impact parameter resolution, including Layer 00 , arrives to $40 \mu \mathrm{m}$ at $p_{T}>3 \mathrm{GeV} / \mathrm{c}$.

Surrounding the silicon detector is the Central Outer Tracker (COT) [5], the anchor of the CDF Run II tracking system. It is a $3.1 \mathrm{~m}$ long cylindrical drift chamber that covers the radial range from 40 to $137 \mathrm{~cm}(|\eta|<1)$. The COT contains 96 sense wire layers, which are radially grouped into eight "superlayers", as inferred from the end plate section shown in figure 3.6. 


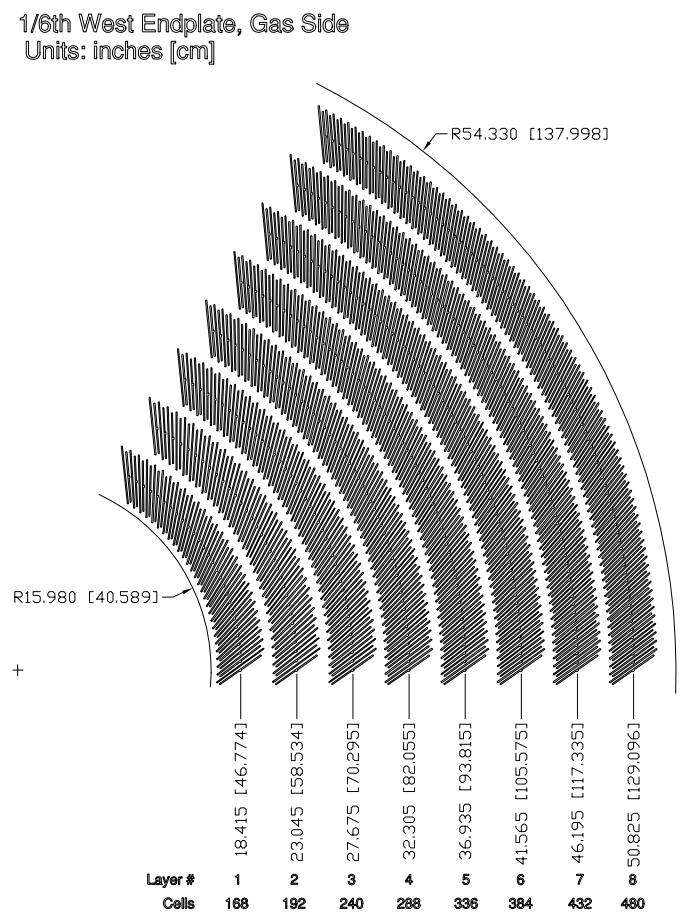

Figure 3.6: Layout of wire planes on a COT endplate.

Each superlayer is divided in $\phi$ into "supercells", and each supercell has 12 sense wires and a maximum drift distance that is approximately the same for all superlayers. Therefore, the number of supercells in a given superlayer scales approximately with the radius of the superlayer. The entire COT contains 30,240 sense wires. Approximately half the wires run along the $z$ direction ("axial"). The other half are strung at a small angle $\left(2^{\circ}\right)$ with respect to the $z$ direction ("stereo"). The combination of the axial and stereo information allows us to measure the $z$ positions. Particles originated from the interaction point, which have $|\eta|<1$, pass through all 8 superlayers of the COT.

The supercell layout, shown in figure 3.7 for superlayer 2, consists of a wire plane containing sense and potential wires, for field shaping and a field (or cathode) sheet on either side. Both the sense and potential wires are $40 \mu \mathrm{m}$ diameter gold plated tungsten. The field sheet is $6.35 \mu \mathrm{m}$ thick Mylar with vapor-deposited gold on both sides. Each field sheet is shared with the neighboring supercell.

The COT is filled with an Argon-Ethane gas mixture and Isopropyl alcohol (49.5:49.5:1). The mixture is chosen to have a constant drift velocity, approximately $50 \mu \mathrm{m} / \mathrm{ns}$ across the cell width and the small content of isopropyl alcohol is intended to reduce the aging and 


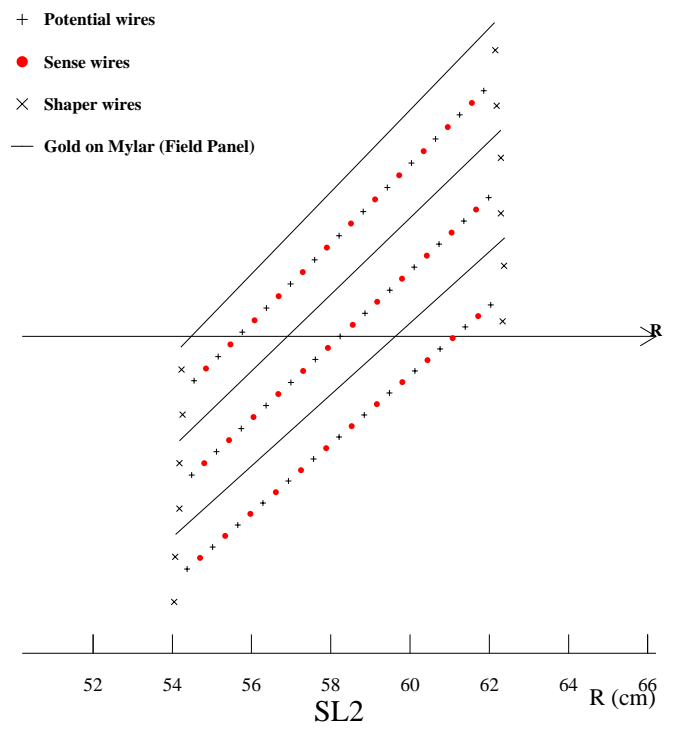

Figure 3.7: Layout of wires in a COT supercell.

build up on the wires. When a charged particle passes through, the gas is ionized. Electrons drift toward the sense wires. Due to the magnetic field that the COT is immersed in, electrons drift at a Lorentz angle of $35^{\circ}$. The supercell is tilted by $35^{\circ}$ with respect to the radial direction to compensate for this effect. The momentum resolution of the tracks in the COT chamber depends on the $p_{T}$ and is measured to be approximately $0.15 \% \mathrm{GeV} / \mathrm{c}^{-1}$, with corresponding hit resolution of about $140 \mu \mathrm{m}$ [6]. In addition to the measurement of the charged particle momenta, the COT is used to identify particles, with $p_{T}>2 \mathrm{GeV}$, based on $\mathrm{dE} / \mathrm{dx}$ measurements.

Just outside the tracking system, CDF II has a Time of Flight (TOF) detector [7]. It is a barrel of scintillator almost $3 \mathrm{~m}$ long located at $140 \mathrm{~cm}$ from the beam line with a total of 216 bars, each covering $1.7^{\circ}$ in $\phi$ and pseudorapidity range $|\eta|<1$. Particle identification is achieved by measuring the time of arrival of a particle at the scintillators with respect to the collision time. Thus, combining the measured time-of-flight and the momentum and path length, measured by the tracking system, the mass of the particle can then determined. The resolution in the time-of-flight measurement is $\approx 100 \mathrm{ps}$ and it provides at least two standard deviation separation between $K^{ \pm}$and $\pi^{ \pm}$for momenta $p<1.6 \mathrm{GeV} / \mathrm{c}$.

As a summary, figure 3.8 illustrates the Tracking and Time of Flight systems. 


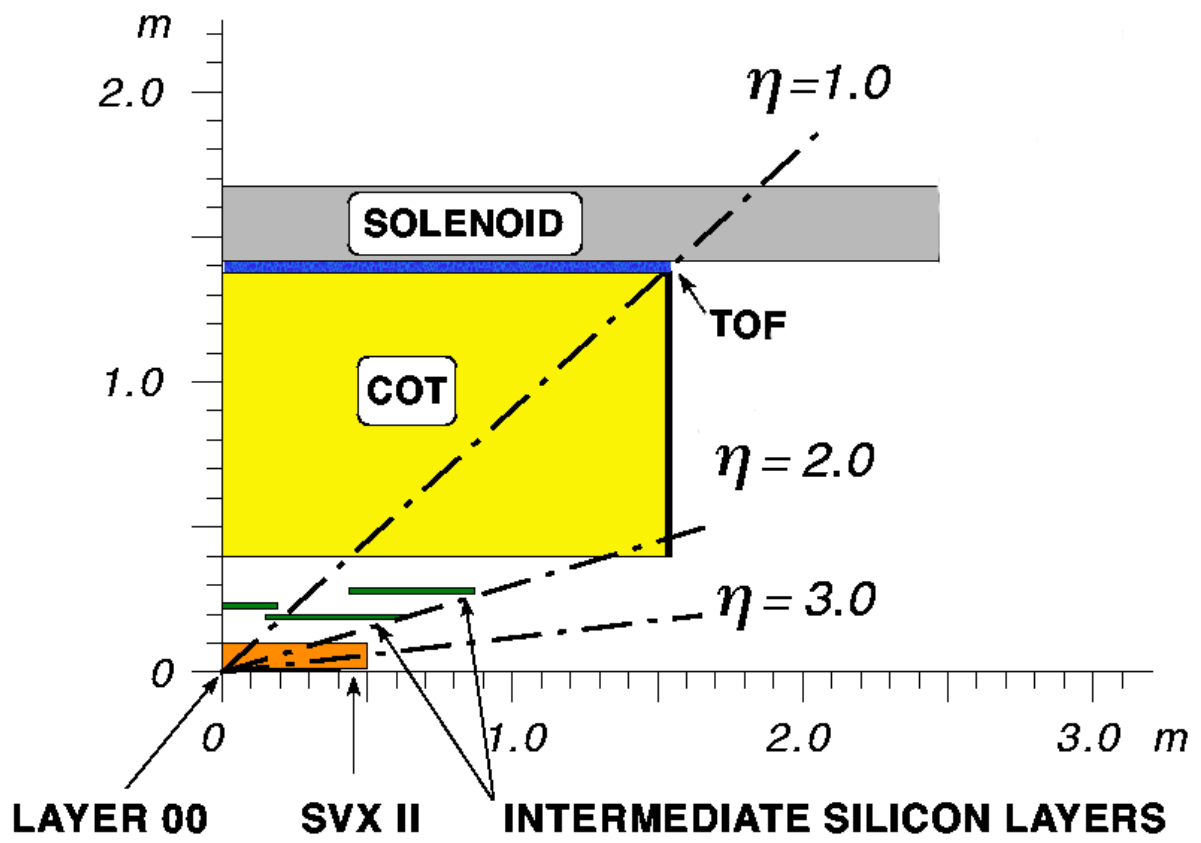

Figure 3.8: The CDF II tracker layout showing the different subdetector systems.

\subsubsection{Calorimeter system}

Surrounding the CDF tracking volume, outside of the solenoid coil, there is the calorimeter system. The different calorimeters that compose the system are scintillator-based detectors and segmented in projective towers (or wedges), in $\eta \times \phi$ space, that point to the interaction region. The total coverage of the system is $2 \pi$ in $\phi$ and about $|\eta|<3.64$ units in pseudorapidity.

The calorimeter system is divided in two regions: central and plug. The central calorimeter covers the region $|\eta|<1.1$ and is split into two halves at $|\eta|=0$. The forward plug calorimeters cover the angular range corresponding to $1.1<|\eta|<3.64$, as it is shown in figure 3.9. Due to this structure two "gap" regions are found at $|\eta|=0$ and $|\eta| \sim 1$.1.

\section{Central Calorimeters}

The central calorimeters consist of 478 towers, each one is $15^{\circ}$ in azimuth by about 0.11 in pseudorapidity. Each wedge consists of an electromagnetic component backed by a hadronic section. In the central electromagnetic calorimeter (CEM) [8], the scintillators are interleaved with lead layers. The total material has a depth of 18 radiation lengths $\left(X_{0}\right)^{4}$. The

\footnotetext{
${ }^{4}$ The radiation length $X_{0}$ describes the characteristic amount of matter transversed, for high-energy electrons to lose all but $1 / e$ of its energy by bremsstrahlung, which is equivalent to $\frac{7}{9}$ of the length of the mean free path for pair
} 


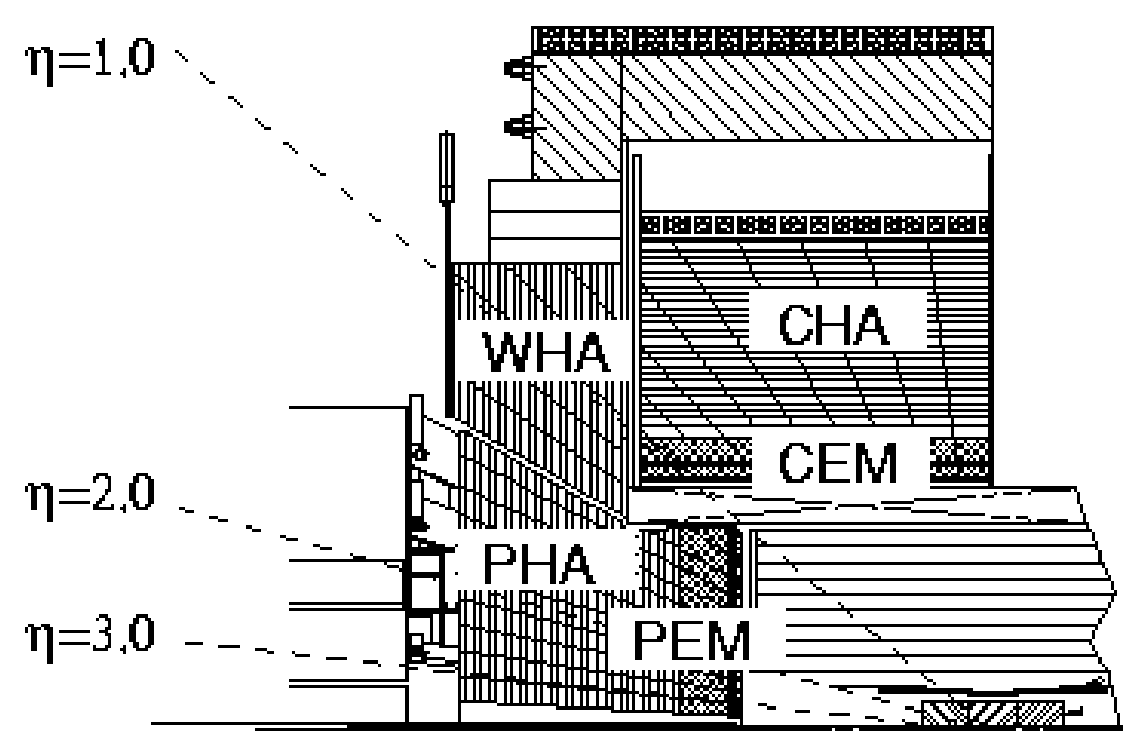

Figure 3.9: Elevation view of $1 / 4$ of the CDF detector showering the components of the CDF calorimeter: CEM, CHA, WHA, PEM and PHA.

central hadronic section (CHA) [9] has alternative layers of steel and scintillator and is 4.7 interaction lengths deep $\left(\lambda_{0}\right)^{5}$. The endwall hadron calorimeter (WHA), with similar construction to CHA, is located with half of the detector behind the CEM/CHA and the other half behind the plug calorimeter. The function of the WHA detector is to provide a hadronic coverage in the region $0.9<|\eta|<\mathbf{1 . 3}$. In the central calorimeter the light from the scintillator is redirected by two wavelength shifting (WLS) fibers, which are located on the $\phi$ surface between wedges covering the same pseudorapidity region, up through the lightguides into two phototubes (PMTs) per tower.

The energy resolution for each section was measured in the testbeam and, for a perpendicular incident beam, it can be parameterized as:

$$
(\sigma / E)^{2}=\left(\sigma_{1} / \sqrt{E}\right)^{2}+\left(\sigma_{2}\right)^{2}
$$

where the first term comes from sampling fluctuations and the photostatistics of PMTs, and the second term comes from the non-uniform response of the calorimeter. In the CEM, the energy resolution for high energy electrons and photons is $\frac{\sigma\left(E_{T}\right)}{E_{T}}=\frac{13.5 \%}{\sqrt{E_{T}}} \oplus 1.5 \%$, where $E_{T}=E \sin \theta$ being $\theta$ the beam incident angle. Charge pions were used to obtain the energy

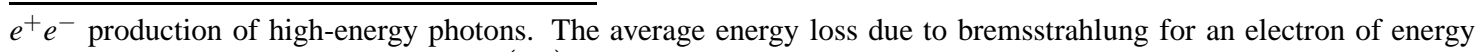
$\mathrm{E}$ is related to the radiation length by $\left(\frac{d E}{d x}\right)_{b r e m s}=-\frac{E}{X_{0}}$ and the probability for an electron pair to be created by a high-energy photon is $\frac{7}{9} X_{0}$.

${ }^{5} \mathrm{An}$ interaction length is the average distance a particle will travel before interacting with a nucleus: $\lambda=\frac{A}{\rho \sigma N_{A}}$, where $A$ is the atomic weight, $\rho$ is the material density, $\sigma$ is the cross section and $N_{A}$ is the Avogadro's number. 
resolution in the CHA and WHA detectors that are $\frac{\sigma\left(E_{T}\right)}{E_{T}}=\frac{50 \%}{\sqrt{E_{T}}} \oplus 3 \%$ and $\frac{\sigma\left(E_{T}\right)}{E_{T}}=\frac{75 \%}{\sqrt{E_{T}}} \oplus 4 \%$, respectively.

Plug Calorimeters

One of the major components upgraded for the Run II was the plug calorimeter [10]. The new plug calorimeters are built with the same technology as the central components and replace the Run I gas calorimeters in the forward region. The $\eta \times \phi$ segmentation depends on the tower pseudorapidity coverage. For towers in the region $|\eta|<2.1$, the segmentation is $7.5^{\circ}$ in $\phi$ and from 0.1 to 0.16 in the pseudorapidity direction. For more forward wedges, the segmentation changes to $15^{\circ}$ in $\phi$ and about 0.2 to 0.6 in $\eta$.

As in the central calorimeters, each wedge consists of an electromagnetic (PEM) and a hadronic section (PHA). The PEM, with 23 layers composed of lead and scintillator, has a total thickness of about $21 X_{0}$. The PHA is a steel/scintillator device with a depth of about $7 \lambda_{0}$. In both sections the scintillator tiles are read out by WLS fibers embedded in the scintillator. The WLS fibers carry the light out to PMTs tubes located on the back plane of each endplug. Unlike the central calorimeters, each tower is only read out by one PMT.

Testbeam measurements determined that the energy resolution of the PEM for electrons and photons is $\frac{\sigma}{E}=\frac{16 \%}{\sqrt{E}} \oplus 1 \%$. The PHA energy resolution is $\frac{\sigma}{E}=\frac{80 \%}{\sqrt{E}} \oplus 5 \%$ for charged pions that do not interact in the electromagnetic component. Table 3.2 summarizes the calorimeter subsystems and their characteristics.

\begin{tabular}{|l|c|c|c|}
\hline Calorimeter & Coverage & Thickness & Energy resolution (E expressed in GeV) \\
\hline CEM & $|\eta|<1.1$ & $18 X_{0}$ & $\frac{13.5 \%}{\sqrt{E_{T}}} \oplus 2 \%$ \\
CHA & $|\eta|<0.9$ & $4.7 \lambda_{0}$ & $\frac{50 \%}{\sqrt{E_{T}}} \oplus 3 \%$ \\
WHA & $0.9<|\eta|<1.3$ & $4.7 \lambda_{0}$ & $\frac{75 \%}{\sqrt{E_{T}}} \oplus 4 \%$ \\
\hline PEM & $1.1<|\eta|<3.6$ & $21 X_{0}, 1 \lambda_{0}$ & $\frac{16 \%}{\sqrt{E}} \oplus 1 \%$ \\
PHA & $1.2<|\eta|<3.6$ & $7 \lambda_{0}$ & $\frac{80 \%}{\sqrt{E}} \oplus 5 \%$ \\
\hline
\end{tabular}

Table 3.2: CDF II Calorimeter subsystems and characteristics. The energy resolution for the EM calorimeter is given for a single incident electron and that for the hadronic calorimeter for a single incident pion.

The central and forward parts of the calorimeter have their own shower profile detectors: shower maximum and preshower detectors. The Central Shower Maximum (CES) and the Plug Shower Maximum (PES) are positioned at about $6 X_{0}$, while the Central Preradiator (CPR) and the Plug Preradiator (PPR) are located at the inner face of the calorimeters. These detectors help on particle identification, separating $e^{ \pm}, \gamma_{S}$ and $\pi^{0} s$. 


\subsubsection{Muons system}

The muon system, which consists of sets of drift chambers and scintillators, is installed beyond the calorimetry system as the radially outermost component of CDF Run II detector $(\mathbf{r} \sim 3.5 \mathrm{~m})$. The muon system $[11,12]$ is divided into different subsystems, that cover the pseudorapidity range $|\eta|<2.0$ : the Central Muon Detector (CMU), the Central Muon Upgrade Detector (CMP/CSP), the Central Muon Extension Detector (CMX/CSX) and the Intermediate Muon Detector (IMU). The $z$ and $\phi$ coordinates of the muon candidate are often provided by the chambers while the scintillator detectors are used for triggering and spurious signal rejection.

\subsection{Luminosity Measurement}

\subsubsection{CLC detector}

In CDF, the beam luminosity is determined using gas Cherenkov counters (CLC) [13] located in the pseudorapidity region $3.7<|\eta|<4.7$, which measure the average number of inelastic interaction per bunch crossing. Each module consists of 48 thin, gas-filled, Cherenkov counters. The counters are arranged around the beam pipe in three concentric layers, with 16 counters each, and pointing to the center of the interaction region. The cones in the two outer layers are about $180 \mathrm{~cm}$ long and the inner layer counters, closer to the beam pipe, have a length of $110 \mathrm{~cm}$. The Cherenkov light is detected with photomultiplier tubes.

\subsubsection{Measurement of the luminosity}

The average number of primary interactions, $\mu$, is related to the instantaneous luminosity, $\mathcal{L}$, by the expression:

$$
\mu \cdot f_{b c}=\sigma_{t o t} \cdot \mathcal{L}
$$

where $f_{b c}$ is the bunch crossings frequency at Tevatron, on average $1.7 \mathrm{MHz}$ for $36 \times 36$ bunch operations, and $\sigma_{t o t}$ is the total $p \bar{p}$ cross section.

Since the CLC is not sensitive at all to the elastic component of the $p \bar{p}$ scattering, the equation 3.3 can be rewritten using the inelastic cross section, $\sigma_{i n}$, as: 


$$
\mathcal{L}=\frac{\mu \cdot f_{b c}}{\sigma_{i n}}
$$

where now $\mu$ is the average number of inelastic $p \bar{p}$ interactions. The method used in CDF for the luminosity measurement is based on the counting of empty crossings [14]. This method determines $\mu$ by measuring the first bin of the distribution which corresponds to the probability of having zero inelastic interactions, $P_{0}$, through the relation:

$$
P_{0}(\mu)=e^{-\mu}
$$

which is correct if the acceptance of the detector and its efficiency were $100 \%$. In practice, there are some selection criteria, $\alpha$, to define an "interaction". An "interaction" is defined as a $p \bar{p}$ crossing with hits above a fixed threshold on both sides of the CLC detector. Therefore, an empty crossing is a $p \bar{p}$ crossing with no interactions. Given these selection criteria, the experimental quantity $P_{0}$, called $P_{0}^{\exp }\{\alpha\}$, is related to $\mu$ as:

$$
P_{0}^{e x p}\{\mu ; \alpha\}=\left(e^{\varepsilon_{\omega} \cdot \mu}+e^{-\varepsilon_{e} \cdot \mu}-1\right) \cdot e^{-\left(1-\varepsilon_{0}\right) \cdot \mu},
$$

where the acceptances $\varepsilon_{0}$ and $\varepsilon_{\omega / e}$ are, respectively, the probability to have no hits in the combined east and west CLC modules and the probability to have at least one hit exclusively in west/east CLC module. The evaluation of these parameters is based on Monte Carlo simulations, and typical values are $\varepsilon_{0}=0.07$ and $\varepsilon_{\omega / e}=\mathbf{0 . 1 2}$.

To obtain the luminosity measurement using the equation 3.4, the value of $\sigma_{\text {in }}$ is still needed. At the beginning of Run II, an extrapolation to $2 \mathrm{TeV}$ of the value measured at $\sqrt{s}=1.8$ TeV by CDF [15] was used. The cross section would be $\sigma_{i n}=60.4 \mathrm{mb}$. To facilitate the comparison of CDF and D0 cross section measurements in Run II, the collaborations agreed to use a common inelastic cross section [16], $\sigma_{i n}=59.3 \mathrm{mb}$ that is about $1.9 \%$ smaller than previous value. Since CDF never modified the value used online and offline, the CDF quoted luminosity is multiplied offline by a factor of 1.019 .

Different sources of uncertainties have been taken into account to evaluate the systematic uncertainties on the luminosity measurement [17]. The dominated contributions are related to the detector simulation and the event generator used, and have been evaluated to be about 3\%. The total systematic uncertainty in the CLC luminosity measurements is $5.8 \%$, which includes uncertainties on the measurement (4.2\%) and on the inelastic cross section value $(4 \%)$. 


\subsection{Trigger and Data Acquisition}

The average interaction rate at the Tevatron is $1.7 \mathrm{MHz}$ for $36 \times 36$ bunches. In fact, the actual interaction rate is higher because the bunches circulate in three trains of 12 bunches in each group spaced $396 \mathrm{~ns}$ which leads to a crossing rate of $2.53 \mathrm{MHz}$. The interaction rate is orders of magnitude higher than the maximum rate that the data acquisition system can handle. Furthermore, the majority of collisions are not of interest. This leads to implementation of a trigger system that preselects events online and decides if the corresponding event information is written to tape or discarded.

The CDF trigger system consists of three trigger levels, see figures 3.10 and 3.11, where the first two levels are hardware based and the third one is a processor farm. The decisions taken by the system are based on increasingly more complex event information. The two hardware levels are monitored and controlled by the Trigger Supervisor Interface (TSI), which distributes signals from the different sections of the trigger and DAQ system, a global clock and bunch crossing signal.

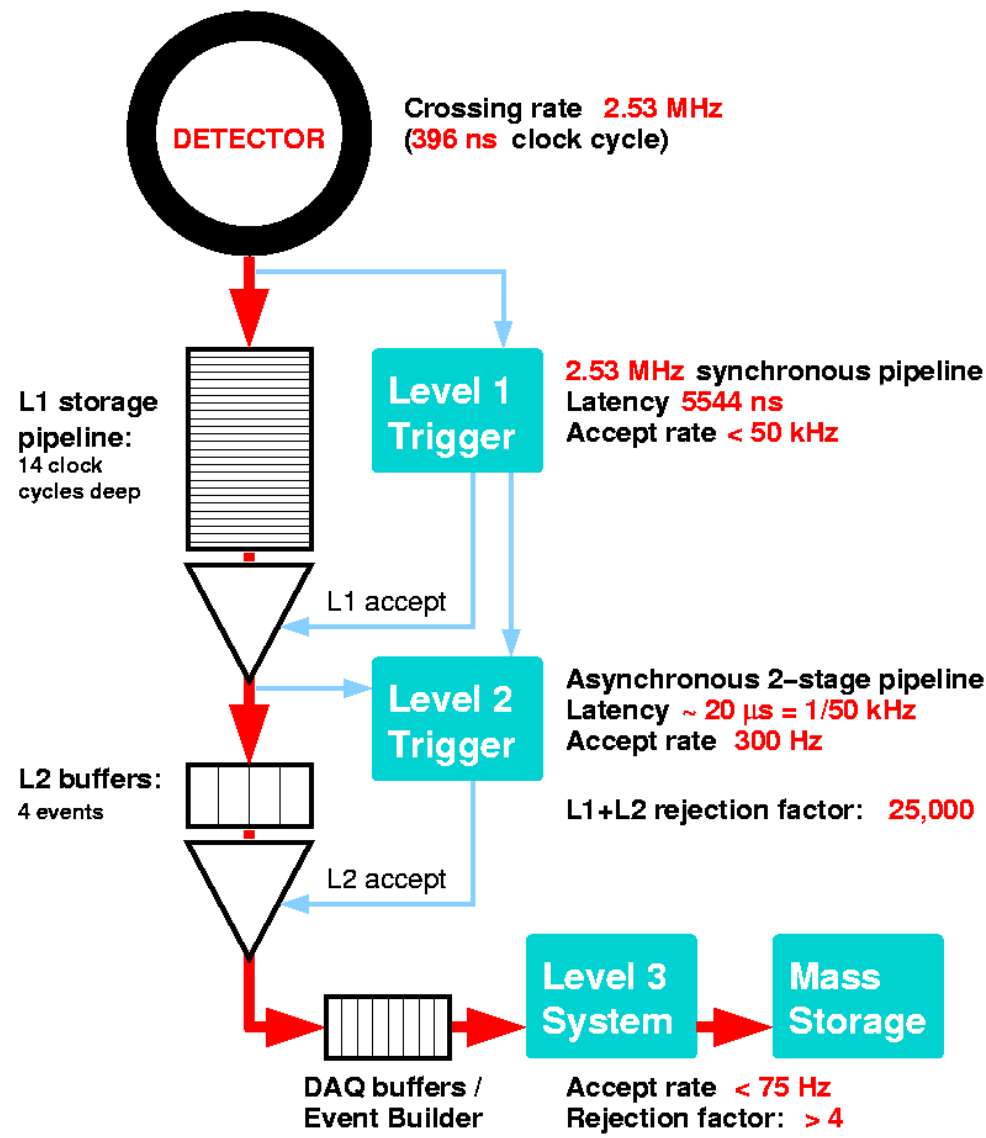

Figure 3.10: Block diagram showing the global trigger and DAQ systems at CDF II. 


\section{RUN II TRIGGER SYSTEM}

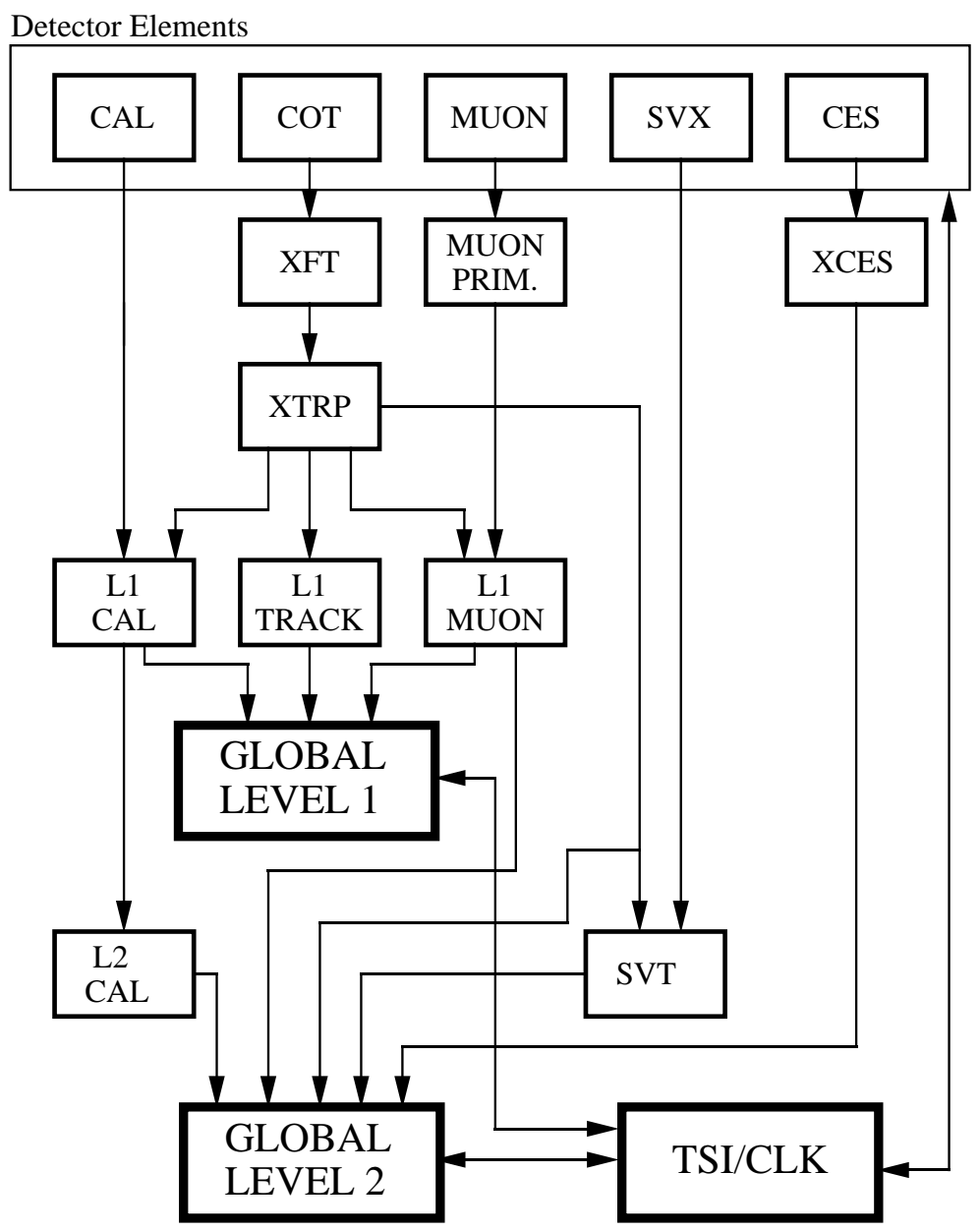

PJW 9/23/96

Figure 3.11: Block diagram showing the Level 1 and Level 2 trigger systems. 


\subsubsection{Level 1 trigger}

The Level 1 trigger is a synchronous system with an event read and a decision made every beam crossing. The depth of the L1 decision pipeline is approximately $4 \mu \mathrm{s}$ (L1 latency). The $\mathrm{L} 1$ buffer must be at least as deep as this processing pipeline or the data associated with a particular $\mathrm{L} 1$ decision would be lost before the decision is made. The L1 buffer is 14 crossings deep (5544 $\mathbf{n s}$ at $396 \mathrm{~ns}$ bunch spacing) to provide a margin for unanticipated increases in $\mathrm{L1}$ latency. The Level 1 reduces the event rates from $2.53 \mathrm{MHz}$ to less than $50 \mathrm{kHz}$.

The Level 1 hardware consists of three parallel processing streams which feed inputs of the Global Level 1 decision unit. One stream finds calorimeter based objects (L1 CAL), another finds muons (L1 MUON), while the third one finds tracks in the COT (L1 TRACK). Since the muons and the calorimeter based objects require the presence of a track pointing at the corresponding outer detector element, the tracks must be sent to the calorimeter and muon streams as well as the track only stream.

- The L1 CAL calorimeter trigger is employed to detect electrons, photons, jets, total transverse energy and missing transverse energy, $E_{T}^{\text {miss }}$. The calorimeter triggers are divided into two types: object triggers (electron, photons and jets) and global triggers $\left(\sum E_{T}\right.$ and $E_{T}^{m i s s}$ ). The calorimeter towers are summed into trigger towers of $15^{\circ}$ in $\phi$ and by approximately 0.2 in $\eta$. Therefore, the calorimeter is divided in $24 \times 24$ towers in $\eta \times \phi$ space [18]. The object triggers are formed by applying thresholds to individual calorimeter trigger towers, while thresholds for the global triggers are applied after summing energies from all towers.

- The L1 TRACK trigger is designed to detect tracks on the COT. An eXtremely Fast Tracker (XFT) [19] uses hits from 4 axial layers of the COT to find tracks with a $p_{T}$ greater than some threshold $(\sim 2 \mathrm{GeV} / \mathrm{c})$. The resulting track list is sent to the extrapolation box (XTRP)[20] that distributes the tracks to the Level 1 and Level 2 trigger subsystems.

- L1 MUON system uses muon primitives, generated from various muon detector elements, and XFT tracks extrapolated to the muon chambers by the XTRP to form muon trigger objects. For the scintillators of the muon system, the primitives are derived from single hits or coincidences of hits. In the case of the wire chambers, the primitives are obtained from patterns of hits on projective wire with the requirement that the difference in the arrival times of signals be less than a present threshold. This maximum allowed time difference imposes a minimum $p_{T}$ requirement for hits from a single tracks. 
Finally, the Global Level 1 makes the L1 trigger decision based on the quantity of each trigger object passed to it.

\subsubsection{Level 2 trigger}

The Level 2 trigger is an asynchronous system which processes events that have received a L1 accept in FIFO (First In, First Out) manner. It is structured as a two stage pipeline with data buffering at the input of each stage. The first stage is based on dedicated hardware processor which assembles information from a particular section of the detector. The second stage consists of a programmable processors operating on lists of objects generated by the first stage. Each of the $\mathbf{L} 2$ stages is expected to take approximately $10 \mu$ s giving a latency of approximately $20 \mu \mathrm{s}$. The $\mathbf{L} 2$ buffers provide a storage of four events. After the Level 2, the event rate is reduced to about $300 \mathrm{~Hz}$.

In addition of the trigger primitives generated for $\mathrm{L} 1$, data for the $\mathrm{L} 2$ come from the shower maximum strip chambers in the central calorimeter and the $r \times \phi$ strips of the SVX II. There are three hardware systems generating primitives at Level 2: Level 2 cluster finder (L2CAL), shower maximum strip chambers in the central calorimeter (XCES) and the Silicon Vertex Tracker (SVT).

- The L2CAL hardware carries out the hardware cluster finder functions. It receives trigger tower energies from the L1 CAL and applies seed and 'shoulder" thresholds for cluster finding. It is basically designed for jet triggers.

- The shower maximum detector provides a much better spacial resolution than a calorimeter wedge. The XCES boards perform sum of the energy on groups of four adjacent CES wires and compare them to a threshold (around $4 \mathrm{GeV}$ ). This information is matched to XFT tracks to generate a Level 2 trigger. This trigger hardware provides a significant reduction in combinatorial background for electrons and photons.

- Silicon Vertex Tracker [21] uses hits from the $r \times \phi$ strips of the SVX II and tracks from the XFT to find tracks in SVX II. SVT improves on the XFT resolution for $\phi$ and $p_{T}$ and adds a measurement of the track impact parameter $d_{0}$. Hereby the efficiency and resolution are comparable to those of the offline track reconstruction. The SVT enables triggering on displaced tracks, that have a large impact parameter $d_{0}$.

\subsubsection{Level 3 trigger}

When an event is accepted by the Level 2 trigger, its data become available for readout distributed over a couple of hundred of VME Readout Buffers (VRBs). The event has to be 
assembled from pieces of data from the $\mathrm{L} 2$ system into complete events, this is the purpose of the Event Builder. It is divided into 16 sub-farms, each consisting of 12-16 processor nodes. Once the event is built, it is sent to one place in the Level 3 farm. The Level 3 trigger reconstructs the event following given algorithms. These algorithms take advantage of the full detector information and improved resolution not available to the lower trigger levels. This includes a full 3-dimensional track reconstruction and tight matching of tracks to calorimeter and muon-system information. Events that satisfy the Level 3 trigger requirements are then transfered onward to the Consumer Server/Data Logger (CSL) system for storage first on disk and later on tape. The average processing time per event in Level 3 is on the order of one second. The Level 3 leads to a further reduction in the output rate, a roughly $50 \mathrm{~Hz}$.

A set of requirements that an event has to fulfill at Level 1, Level 2 and Level 3 constitutes a trigger path. The CDF II trigger system implements about 150 trigger paths. An event will be accepted if it passes the requirements of any one of these paths and, depending of the trigger path, it will be stored in a trigger dataset. A complete description of the different datasets at CDF Run II can be found in [22].

In addition to impose the trigger requirements to select out interesting physics events, trigger can be prescaled in the different levels. To prescale means to accept only a predetermined fraction of events selected by a given trigger path.

\subsection{B Physics with Silicon Detectors}

The physics program of CDF is rich and wide, including the study of strong and electroweak interactions, as well as being at the high energy frontier, where searches for failures of the Standard Model would indicate the presence of new Physics. One particular field that beneficts particularly from silicon detectors and will be discussed below is $B$-physics. Traditionally, $B$ physics has been the domain of $e^{+} e^{-}$machines operating on the $\Upsilon(4 S)$ resonance or the $Z^{0}$ pole. However, the UA1 collaboration at CERN has shown that $B$ physics is feasible at a hadron collider (see for example Ref. [31]). The first signal of fully reconstructed $B$ mesons at a hadron collider was published by the CDF Collaboration in 1992 [34]. CDF reconstructed $B^{+} \rightarrow \mathrm{J} / \psi K^{+}$events in a data sample of $2.6 \mathrm{pb}^{-1}$ taken during the Tevatron Run 0 at the end of the 1980s. Since then, the experimental techniques improved significantly, especially with the development of high precision silicon vertex detectors and trigger.

\subsubsection{Features of $B$ Physics at a Hadron Collider}

In the Tevatron, the production mode of $B$ hadrons is $p \bar{p} \rightarrow b \bar{b} X$. The main motivation for studying $B$ physics at a hadron collider is the large $b$ quark production cross section 
$\delta_{b} \sim 50 \mu \mathbf{b}$ within the central detector regions. In a typical $B$ event in CDF, no well-defined jet structure is visible and the average multiplicity is about 50 charged tracks including tracks from the 'underlying event' particles. It might appear challenging to find the $B$ decay products in this quite complex environment of a hadronic collision. One way to extract $B$ decays in a $p \bar{p}$ collision is to take advantage of the relatively long life of $B$ hadrons resulting in a $B$ decay vertex which is clearly separated from the primary $p \bar{p}$ interaction vertex by hundred of microns.

An important feature for $B$ physics at a hadron collider is a good tracking capability which is usually achieved with a central tracking chamber. Together with a silicon detector assisting in tracking, an excellent track momentum resolution translates into an excellent invariant mass resolution. In addition to excellent tracking, superb vertexing is the other essential feature of successful $B$ physics studies at a hadron collider For example for a typical $\mathbf{B}$ hadron decay to $\mathrm{J} / \psi \mathbf{X}$, the two muons of the $\mathrm{J} / \psi$ signal candidates are vertexed using tracking information from the silicon detector. The distribution of the two-dimensional distance between the primary $p \bar{p}$ interaction vertex and the reconstructed dimuon vertex shows several features: a prominent peak at zero decay length results from prompt $\mathrm{J} / \psi$ candidates which are produced at the primary interaction vertex and constitute about the $80 \%$ of all $\mathrm{J} / \psi$ candidates. The width of this peak reveals information about the vertexing resolution. At positive decay lengths, $\mathrm{J} / \psi$ mesons from $B$ hadron decays are described by an exponential slope. At a distance of about $100 \mu \mathrm{m}$ from the primary interaction vertex, mainly $\mathrm{J} / \psi$ candidates from $B$ decays remain.

\subsubsection{Triggering on $B$ Decay Products}

The total inelastic $p \bar{p}$ cross section at the Tevatron is about three orders of magnitude larger than the $b$ production cross section. The CDF trigger system is therefore the most important tool for finding $B$ decay products. In addition, the cross section for $b$ quark production is steeply falling. It drops by almost two orders of magnitude between a $b$ quark of transverse momentum $\left(p_{T}\right)$ of about $8 \mathrm{GeV} / c$ and $25 \mathrm{GeV} / c$. To find $B$ decay products in hadronic collisions, it is desirable to go as low as possible in the decay products transverse momentum, exploiting as much as possible of the steeply falling $b$ cross section. Of course, the limiting factor is the bandwidth of the experiments' data acquisition system.

In Run I, all the $B$ physics triggers at CDF and DO were based on leptons including single and dilepton triggers. In Run II, both experiments still exploit heavy flavour decays which leave leptons in the final state. Identification of dimuon events down to very low momentum is possible, allowing for efficient $\mathrm{J} / \psi \rightarrow \mu^{+} \mu^{-}$triggers, and as a consequence both experiments are able to fully reconstruct $B$ decay modes involving $\mathrm{J} / \psi$. Both experiments 
also use inclusive lepton triggers designed to accept semileptonic $B \rightarrow l v_{l} X$ decays. DO has an inclusive muon trigger with excellent acceptance, allowing them to accumulate very large samples of semileptonic decays.

New to the CDF detector is the ability to select events based upon track impact parameter. The Silicon Vertex Trigger (SVT) gives CDF access to purely hadronic $B$ decays and makes CDF's $B$ program fully competitive with the one at the $e^{+} e^{-} B$ factories. The hadronic track trigger is the first of its kind operating successfully at a hadron collider. It works as follows: with a fast track trigger at Level 1, CDF finds track pairs in the COT with $p_{T}>1.5$ $\mathrm{GeV} / c$. At trigger Level 2, these tracks are linked into the silicon vertex detector and cuts on the track impact parameter (e.g. $d>100 \mu \mathrm{m})$ are applied. The SVT track impact parameter resolution is about $50 \mu \mathrm{m}$ including a $33 \mu \mathrm{m}$ contribution from the transverse beam spreading. The original motivation for CDF's hadronic track trigger was to select $B^{0} \rightarrow \pi \pi$ decays to be used for $C P$ violation studies. The $\mathrm{CDF}$ semileptonic triggers require an additional displaced track associated with the lepton, providing cleaner samples with smaller yields.

\subsubsection{Selected $B$ Physics Results from the Tevatron}

With the different $B$ trigger strategies above, the Collider experiments are able to trigger and reconstruct large samples of heavy flavour hadrons. To give an idea about the sample sizes available for heavy flavour analyses, the approximate yield for $D^{0} \rightarrow K^{-} \pi^{+}$is $\sim 6000$ events per $\mathrm{pb}^{-1}$, for $B^{-} \rightarrow D^{0} \pi^{-}$it is $\sim 16$ events, for $\mathrm{J} / \psi \rightarrow \mu^{+} \mu^{-}$it is $\sim 7000$ events, for $B^{-} \rightarrow \mathrm{J} / \psi K^{-}$it is $\sim 16$ events or for $B \rightarrow D l v$ it is $\sim 400$ events per $\mathrm{pb}^{-1}$ at the Tevatron. In the following, some selected $B$ physics results from CDF are discussed. As already stressed, those results were possible due to a reliable performance of the silicon detectors.

\subsubsection{1 $B$ Hadron Masses and Lifetimes}

Measurements of $B$ hadron masses and lifetimes are basic calibration measures to demonstrate the understanding of heavy flavour reconstruction. For example, CDF uses exclusive $B$ decay modes into $\mathrm{J} / \psi$ mesons for precision measurements of $B$ hadron masses reconstruction decay modes $B^{0} \rightarrow \mathbf{J} / \psi K^{* 0}, B^{+} \rightarrow \mathbf{J} / \psi K^{+}, B_{s}^{0} \rightarrow \mathbf{J} / \psi \phi$, and $\Lambda_{b} \rightarrow \mathbf{J} / \psi \Lambda$. These modes combine good signal statistics with little background.

The proper time of a $B$ decay is determined from the distance between the primary vertex of the $p \bar{p}$ collision and the $B$ meson decay vertex measured in the plane transverse to the beam axis:

$$
L_{x y}^{B}=\left(\vec{x}_{B}-\vec{x}_{p r i m}\right) \cdot \vec{p}_{T} /\left|\vec{p}_{T}\right|,
$$


where $\vec{p}_{T}$ is the measured transverse momentum vector. The typical resolution of $L_{x y}^{B}$ is $40 \mu \mathrm{m}$. CDF uses the run-averaged beam position whose contribution to the $L_{x y}^{B}$ uncertainty is $\sim 30 \mu \mathrm{m}$. In the case of fully reconstructed $B$ hadron decays, the proper lifetime $\tau$ is obtained by $c \tau=L_{x y}^{B} \cdot M_{B} / p_{T}$, where $M_{B}$ is the $B$ hadron mass. In the case of inclusive decays where the $B$ hadron is not fully reconstructed, a boost correction obtained from a Monte Carlo simulation is usually applied. CDF has measured the lifetimes of the $B^{+}, B^{0}$ and $\Lambda_{b}$ hadrons from channels $B^{+} \rightarrow J / \Psi K^{+}, B^{0} \rightarrow J / \Psi K^{* 0}, J / \Psi K_{s}^{0}$ and $\Lambda_{b} \rightarrow J / \Psi \Lambda$ [32] .

\subsubsection{Prompt Charm Cross Section}

Previous Run I measurements of the $b$ production cross section at the Tevatron have consistenly been higher than the Next-to-Leading-Order (NLO) QCD predictions. Although the level of discrepancy has been reduced with recent theoretical activity, it is not yet clear that the entire scope of the problem is understood. Both experiments CDF and DO measured again the $b$ and $b \bar{b}$ cross sections in run II. To further shed light on this problem, CDF presented a measurement of the charm production cross section [36]. Using the secondary vertex trigger, CDF has been able to reconstruct very large samples of charm decays.

Since the events are accepted based upon daughter tracks with large impact parameter, the sample of reconstructed charm decays contains charm from direct $c \bar{c}$ production, as well as charm from $B$ hadron decays $b \rightarrow c$. To extract the charm meson cross section, it is necessary to extract the fraction of $D$ mesons that are coming from prompt charm production and remove the fraction from $b \rightarrow c$ decays. This is done by measuring the impact parameter of the charm meson. If it arises from direct $c \bar{c}$ production, the charm meson will have a small impact parameter pointing back to the primary $p \bar{p}$ interaction vertex. If the charm meson originates from $B$ decays, it will typically not extrapolate back to the primary vertex. Using this technique, along with a sample of $K_{S}^{0} \rightarrow \pi^{+} \pi^{-}$decays for calibration, CDF found that $\mathbf{8 0 - 9 0} \%$ of the charm mesons originate from direct charm production. The shorter charm lifetime is compensated enough by the copious charm production in hadronic collisions.

\subsubsection{Hadronic Branching Ratios}

Two-body Charmless $B$ Decay. With the SVT trigger, CDF measured $B$ decays with nonleptonic final states. One set of modes of particular interest are rare charmless two-body decays as they are potential modes for $C P$ violation mesurements. Requiring the final state to consist of two charged hadrons $\left(B \rightarrow h h\right.$ ), the following modes can be accessed: $B^{0} \rightarrow \pi^{+} \pi^{-}$, $B^{0} \rightarrow K^{ \pm} \pi^{\mp}, B_{S}^{0} \rightarrow K^{ \pm} \pi^{\mp}$, and $B_{S}^{0} \rightarrow K^{+} K^{-}$. The $B^{0}$ states are also reconstructed at the $e^{+} e^{-}$ $B$ factories but the $B_{S}^{0}$ modes are exclusive to the Tevatron. 
$\Lambda_{b} \rightarrow \Lambda_{c} \pi$ Branching Ratio. Using the SVT trigger, CDF has also measured purely hadronic $b$-baryon states [33]. In the decay $\Lambda_{b} \rightarrow \Lambda_{c} \pi^{-}$, with $\Lambda_{c} \rightarrow p K^{-} \pi^{+}$, the reconstructed invariant mass displays an interesting background structure, with almost no background above the $\Lambda_{b}$ peak and a background that rises steeply going to lower mass. This structure is somewhat unique to baryon modes, which are the most massive weakly decaying $B$ hadron states. Because the SVT trigger specifically selects long-lived states, most of the backgrounds are coming from other heavy flavour ( $b$ and $c$ ) decays. Since there are no weakly decaying $B$ hadrons more massive than the $\Lambda_{b}$, there is very little background above the peak. On the other hand, going to masses below the peak, lighter $B$ mesosn begin to contribute. The background in this mode is growing at lower masses because there is more phase space for $B^{+}$, $B^{0}$, and $B_{S}^{0}$ decay modes to contribute.

\subsubsection{Rare Decays}

Flavour changing neutral currents (FCNC) are prohibited on tree-level in the Standard Model (SM). Contributions from sources beyond the SM might measurably enhance the low SM branching fractions of these rare decays. This explains the considerable theoretical interest in $B_{S}^{0} \rightarrow \mu^{+} \mu^{-}$. In some models, non-SM contributions are large enough to allow an observation of this decay mode in Run II.

Experimentally there is considerable background of direct muon pairs in the spectrum of reconstructed muons. This is reduced by requiring the two muon tracks to form a displaced vertex and selecting candidates with a minimum transverse momentum of $p_{T}>\mathbf{4 . 0}$ $\mathrm{GeV} / c$, in addition to the requirement that each muon is isolated. After applying these cuts to the DO analysis, three $B_{S}^{0}$ candidates remained, a result consistent with a background expectation of 3.4 events. DO obtained a limit on the branching ratio of $B R\left(B_{S}^{0} \rightarrow \mu^{+} \mu^{-}\right)<$ $1.6 \cdot 10^{-6}$ at the $90 \%$ confidence limit, this result being competitive with the CDF Run I limit.

Using data selected with the dimuon trigger, CDF has also searched for the flavourchanging neutral current decay $B_{S}^{0} \rightarrow \mu^{+} \mu^{-}$[37]. After applying optimized selection criteria, one event remained in the $B_{S}^{0}$ search window. This yielded an improved upper limit on the branching fraction of $4.3 \times 10^{-8}$ at the $95 \%$ confidence level. This is more than a factor of two improvement over the previous limit produced by CDF in Run I. In addition, an upper limit on the branching fraction of $B^{0} \rightarrow \mu^{+} \mu^{-}$was derived simultaneously yielding values of $7.6 \times 10^{-9}$ at the $95 \%$ confidence level.

Data selected with the displaced track trigger were used to improve the limit on the branching fraction of the FCNC decay $D^{0} \rightarrow \mu^{+} \mu^{-}$[38]. This search begins by reconstructing a clean sample of the kinematically similar $D^{0} \rightarrow \pi^{+} \pi^{-}$decays using a $D^{*}$ tag, followed by muon identification to select $D^{0} \rightarrow \mu^{+} \mu^{-}$candidates. The $D^{0} \rightarrow \pi^{+} \pi^{-}$decays serve also 
as normalization mode. A new upper limit of $5.3 \times 10^{-7}$ at the $95 \%$ confidence level was derived from zero candidates in the search window, almost a factor of two better than the previous best limit.

\subsubsection{5 $B_{s}$ Lifetime Difference and Mixing Phase}

In the standard model (SM), the light (L) and heavy (H) eigenstates of the $B_{s}$ system are expected to mix in such a way that the mass and decay width differences between them, $\Delta m_{s}=m_{H}-m_{L}$ and $\Delta \Gamma_{s}=\Gamma_{L}-\Gamma_{H}$, are sizeable. The mixing phase $\phi_{s}^{S M}$ is within the SM predicted to be small, and thus to a good approximation the two mass eigenstates are expected to be $C P$ eigenstates. New phenomena may introduce a non-vanishing mixing phase $\phi_{s}^{N P}$, leading to a reduction of the observed $\Delta \Gamma_{s}$ compared to the SM prediction: $\Delta \Gamma_{s}=\Delta \Gamma_{s}^{S M} \times\left|\cos \left(\phi_{s}^{S M}-\phi_{s}^{N P}\right)\right|$. While the mass difference $\Delta m_{s}$ in the $B_{s}$ system has been recently measured with a high precision, the mixing phase has remained unknown sofar.

Several analysis have been performed at the Tevatron, to access $\Delta \Gamma_{s}$ and $\phi_{S}: B_{S} \rightarrow K^{+} K^{-}$ is a pure $C P$ even state. Assuming a small $C P$ violating phase, the measurement of the lifetime in this final state directly corresponds to the measurement of the lifetime of the $B_{s}($ light), which can then be compared to measurements of lifetimes in flavour specific eigenstates.

The untagged decay rate asymmetry in semileptonic $B_{S}$ decays $\left(A_{S L}^{S}\right)$ is another handle on the mixing parameters of the $B_{s}$ system:

$$
A_{S L}^{s}=\frac{\Delta \Gamma_{s}}{\Delta m_{S}} \tan \left(\phi_{s}\right)
$$

A third approach is the measure of the branching ratio of $B_{s} \rightarrow D_{s}^{(*)} D_{s}^{(*)}$. This decay is predominantly $C P$ even and gives the largest contribution in the lifetime difference between $B_{s}\left(\right.$ heavy) and $B_{s}($ light $)$. The following relation can be obtained:

$$
2 * B R\left(B_{s} \rightarrow D_{s}^{(*)} D_{s}^{(*)}\right) \approx \frac{\Delta \Gamma_{s}}{\cos \left(\phi_{s}\right) \Gamma_{s}}\left[1+O\left(\frac{\Delta \Gamma}{\Gamma_{s}}\right)\right]
$$

where $\Gamma_{s}$ is the average $B_{s}$ decay width.

The decay $B_{s} \rightarrow \mathbf{J} / \psi \phi$, through the quark process $b \rightarrow c \bar{c} s$, gives rise to both $C P$ even and $C P$ odd final states. It is possible to separate the two $C P$ components of this decay, and thus to measure the lifetime difference, through a simultaneous study of the time evolution and the angular distributions of the decay products of the $J / \psi$ and the $\phi$ mesons. Moreover, with a sizeable lifetime difference, there is a sensitivity to the mixing phase through the interference terms between the $C P$ even and the $C P$ odd waves. 


\subsubsection{6 $\quad B_{s}$ Mixing}

The precise determination of the $B_{S}-\bar{B}_{S}$ oscillation frequency $\Delta m_{s}$ from a time-dependent analysis of the $B_{s}-\bar{B}_{s}$ system has been one of the most important goals for heavy flavour physics at Tevatron. This frequency can be used to strongly improve the knowledge of the Cabbibo-Kobayashi-Maskawa (CKM) matrix, and to constraint contributions from new physics.

The probability $P$ for a $B_{s}$ meson produced at time $t=0$ to decay as a $B_{s}\left(\bar{B}_{s}\right)$ at proper time $t>0$, neglecting effects from $C P$ violation as well as possible lifetime difference between the heavy and light $B_{s}^{0}$ mass eigenstates, is given by

$$
P_{ \pm}(t)=\frac{\Gamma_{s}}{2} e^{-\Gamma_{s} t}\left[1 \pm \cos \left(\Delta m_{s} t\right)\right]
$$

where the subscript "*”"(“"-") indicates that the meson decays as $B_{s}\left(\bar{B}_{s}\right)$. Oscillation have been observed and well established in the $B_{d}$ system. The mass difference $\Delta m_{d}$ is measured to be

$$
\Delta m_{d}=0.505 \pm 0.005 \mathrm{ps}^{-1}
$$

In the $B_{s}$ system oscillation have also been established but till winter 2006 all attempts to measure $\Delta m_{s}$ have only yielded a combined lower limit on the mixing frequency of $\Delta m_{s}>$ $14.5 \mathrm{ps}^{-1}$ at $95 \%$ confidence level. Shortly afterwards CDF presented the first precision measurement on $\Delta m_{s}$, with a significance of the signal of about $3 \sigma$ at that time [39]. Some months later the CDF collaboration updated their result using the very same data, but by using improved analysis techniques, it was able to announce the observation of the $B_{s}-\bar{B}_{s}$ mixing frequency $[40]$.

The canonical $B$ mixing analysis proceeds as follows. The $b$ flavour $(b$ or $\bar{b})$ of the $B$ meson at the time of decay) is determined from the charges of the reconstructed decay products in the final state. The proper time at which the decay occurred is determined from the transverse displacement of the $B_{s}$ decay vertex with respect to the primary vertex, and the $B_{s}$ transverse momentum with respect to the proton beam. Finally, the production $b$ flavour must be known in order to classify the $B$ meson as being mixed (production and decay $b$ flavour are different) or unmixed (production and decay $b$ flavour are equal) at the time of its decay. Then the asymmetry can be measured and thus $\Delta m_{s}$ be determined:

$$
A(t) \equiv \frac{N(t)_{\text {unmixed }}-N(t)_{\text {mixed }}}{N(t)_{\text {unmixed }}+N(t)_{\text {mixed }}}=D \cos \left(\Delta m_{s} t\right)
$$

where $N(t)$ are the time-dependent rates for mixed and unmixed $B_{s}$ decays. $D$ is the so-called dilution, a damping term which is related to the imperfect tagging. It is defined as 
$D=1-P_{w}$, where $P_{w}$ is the probability for a wrong tag. The significance $S$ of a mixing signal is given by:

$$
S=\sqrt{\frac{\varepsilon D^{2}}{2}} \sqrt{\frac{S}{S+B}} e^{-\frac{\left(\Delta m_{5} \sigma_{c t}\right)^{2}}{2}}
$$

$S$ and $B$ are the rates of signal and background events respectively. $\varepsilon D^{2}$ is the figure of merit for the flavour tagging, where $\varepsilon$ is the efficiency to actually apply a tag to a given $B_{S}$ candidate. $\sigma_{c t}$ is the proper decay time resolution. Especially at large $\Delta m_{s}$ values, a small $\sigma_{c t}$ resolution is crucial for this analysis. Further information about the mixing phenomenon can be found in Ref. [41]. 



\section{Chapter 4}

\section{The CDF Silicon Detectors}

The Collider Detector at Fermilab (CDF) completed a major detector upgrade for the start of Run IIa of the Tevatron in March, 2001. The upgraded detector (CDFII) is described in detail elsewhere [72]. The baseline CDFII silicon system consists of 6-7 layers, depending on pseudo-rapidty, of double-sided silicon divided into two sub-systems, SVXII and the Intermediate Silicon Layer (ISL). In 1999, an innovative detector consisting of an additional single-sided layer of silicon built onto the beam-pipe was added to the upgrade. Since this detector is located radially inside layer 0 of SVXII, the sub-system is called layer 00 (L00). The Run II silicon detector allows CDF (Figures 4.1 and 4.2) to make precision tracking and displaced vertices measurement (Fig. 4.3). It is one of the largest operating silicon tracking detectors in high energy physics. The detector has 7-8 layers with 722,432 channels spread over approximately $6 \mathrm{~m}^{2}$ of silicon. Each one of 722,432 channels is being constituted by a reverse-biased strip-shaped diode, where a p-type silicon strip $(n+$ in the $z$ side) is implanted over a $300 \mu \mathrm{m}$ thick n-type silicon bulk. The electronic readout and mechanical unity in which those strips are grouped is called module or ladder. In total, there are 5644 readout chips and 704 modules. The CDF silicon sub-detectors: SVX-II, , and Layer Zero-Zero (L00) as shown in Fig. 4.4. The core of the CDF Run II silicon detector is SVX-II. It is approximately 1 meter long with 5 layers of silicon at radii from $2.5 \mathrm{~cm}$ to $10.6 \mathrm{~cm}$. It is divided into three mechanical barrels along its length with electrical readouts at either end of the mechanical barrels. Three of the five silicon layers have axial and $90^{\circ}$ strips while the remaining two have axial and small angle stereo strips at 1.2 degrees. All the sensor layers in SVX have double sided strips. The strip pitch varies from $60 \mu \mathrm{m}$ to $140 \mu \mathrm{m}$. The five different silicon layers are arranged as twelve wedges covering each one $30^{\circ}$ in the $r-\phi$ plane. The silicon readout is based upon this wedge wide symmetry where each wedge is read out in parallel.

The Intermediate Silicon Layer (ISL) was added to extend silicon tracking to high pseu- 


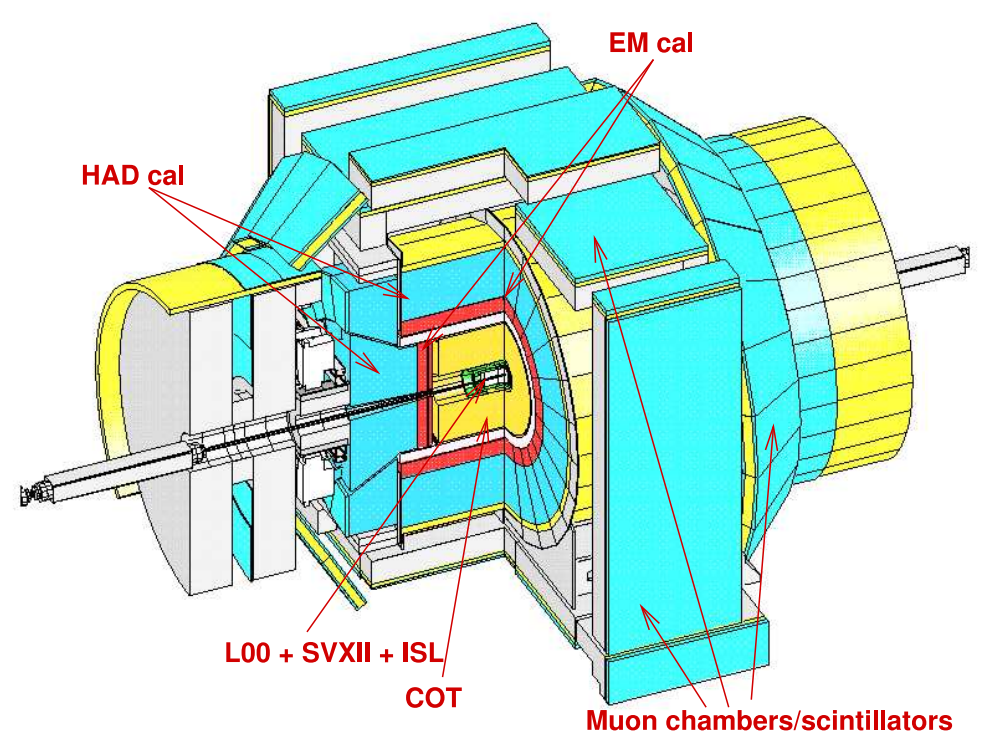

Figure 4.1: The CDF detector. The direction of advance of the protons determines the positive cartesian axis $z$. Its innermost system is the silicon detector (L00+SVXII+ISL). Outside are the Central Outer Tracker (COT), the electromagnetic and hadronic calorimeters (EM cal, HAD cal) and the muon chambers.

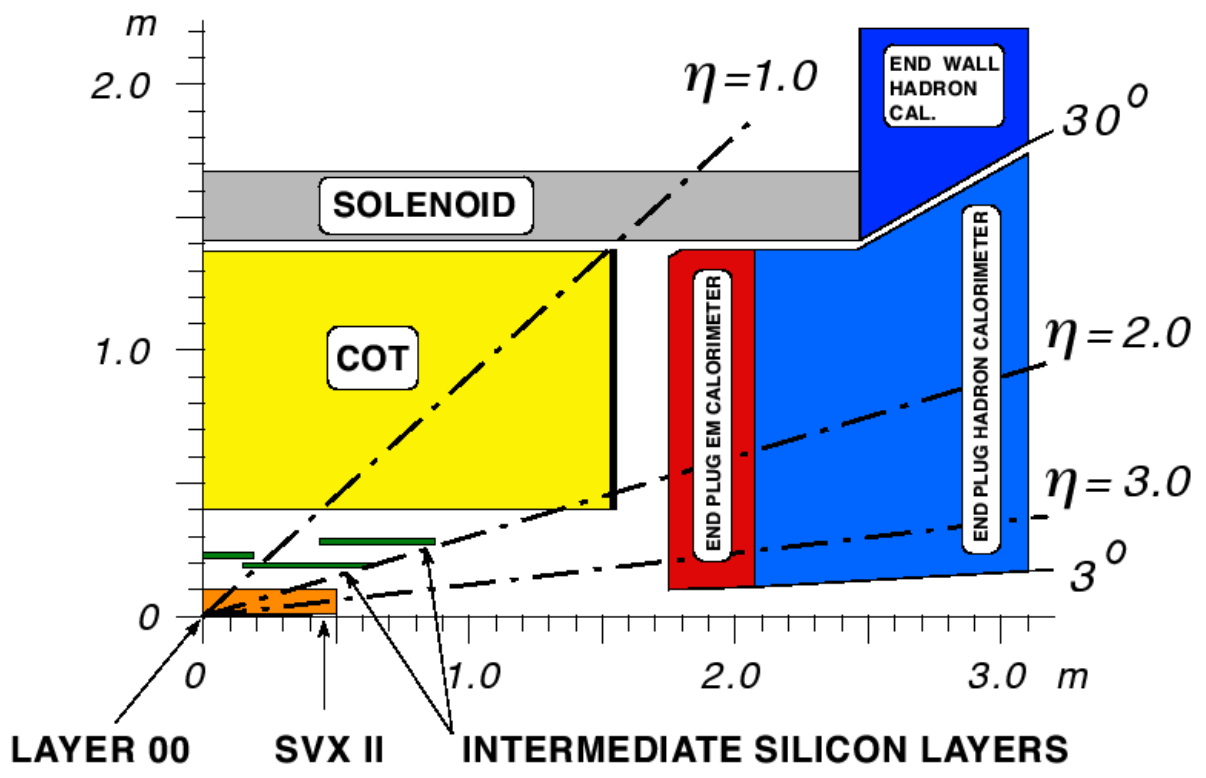

Figure 4.2: Longitudinal section of $\mathrm{CDF}$ and detection ranges for the several subdetectors. The figure shows the first quadrant of the cartesian $Y Z$ plane. The $\eta$ coordinate (pseudo-rapidity) is a transformation of the polar angle $\theta$ under the formula $\eta=-\ln (\tan (\theta / 2))$ 


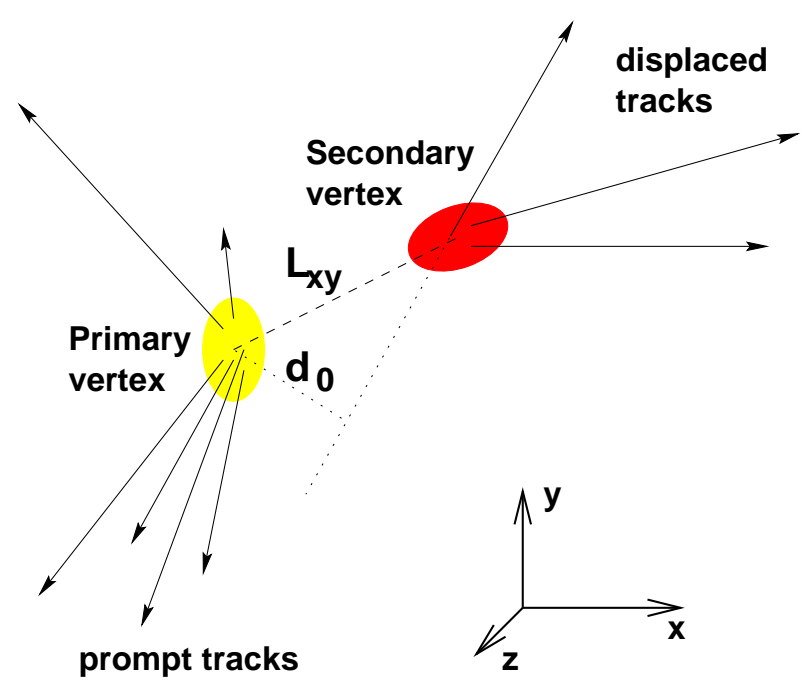

Figure 4.3: Typical lenght between collision vertex and $B$ hadron decay is $L_{x y} \sim 200 \mu \mathrm{m}$. CDF is able to reconstruct vertexes with resolution of about $30 \mu \mathrm{m}$ for high energy particles where multiple scattering can be neglected.

dorapidity and to link tracks between the outer wire chamber and SVX-II as part of an integrated tracking system, as shown in Fig. 4.2. The ISL is composed of one central layer and two outer and forward layers. The ISL layers use double sided small angle $\left(1.2^{\circ}\right)$ stereo strips. The strip pitch for both sides is $112 \mu \mathrm{m}$. The Layer-Zero-Zero (L00) is a single layer of single-sided silicon strips mounted on top of the beam-pipe. This allows precision hit position measurements before scattering by the detector material. Thus the material budget was kept low; 0.6\%-1.0\% of a radiation length. The strip pitch is $25 \mu \mathrm{m}$ but only alternate strips are read out maintaining resolution via charge division while dividing by 2 the number of channels.

During Run II the CDF silicon detectors have been operational for an integrated luminosity of more that $7 \mathrm{fb}^{-1}$. The silicon detectors were originally designed to tolerate $2-3 \mathrm{fb}^{-1}$ before being replaced by a "Run IIb" upgraded detector. This upgrade was cancelled, thus the silicon detectors must now remain operative until the end of Tevatron Run II in 2009 with an expected integrated luminosity of 10-12 $\mathrm{fb}^{-1}$.

\subsection{Description of LOO and SVX-LO}

The detector properties for Layer 00 and the five layers of SVX II are displayed in table 4.1. 


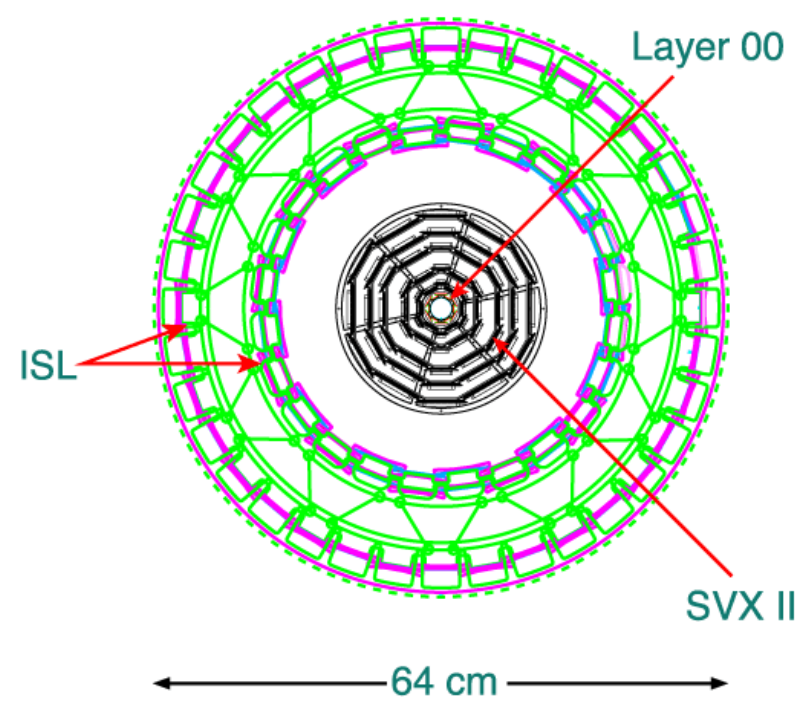

Figure 4.4: Transverse section of the silicon detector showing the three subsystems: L00, SVX-II and ISL.

\subsubsection{L00}

L00 was added to the RunIIa upgrade for two reasons. Firstly, to improve the impact resolution of the CDFII detector. SVXII readout electronics and associated cooling tubes are inside the tracking volume. Multiple scattering in this material degrades impact parameter resolution, especially for low-momentum particles. Placement of a minimal material silicon layer at a smaller radius provides a precise measurement which recovers this lost resolution. Secondly, LOO was installed to extend the useful lifetime of the silicon system, the inner layers of SVXII having a limited lifetime due to radiation damage. Eventually, their doublesided sensors will no longer be depleted at the maximum applicable bias voltage. L00 using single-sided radiation hard silicon can have signicantly higher bias voltage applied and thus can compensate for radiation damaged layers of SVXII.

LO0 is a single castellated [62] layer providing full azimuthal coverage, made up of two concentrical hexagon-shaped sections (see Figs. 4.6, 4.7) located at radii of $1.35 \mathrm{~cm}$ and 1.62 cm (beam-pipe radius is $1.2 \mathrm{~cm}$ ). The silicon sensors are single-sided with a strip pitch of $25 \mu \mathrm{m}$. Alternating strips are read out resulting in an effective pitch of $50 \mu \mathrm{m}$ (a detailed map is shown in Fig. 4.5). The sensors of the inner hexagon are of different size than the sensors of the outer hexagon. The inner hexagon holds 12 sensors (128 strip), two of which are special oxygenated sensors for radiation-hardening (manufactured by Micron), and the rest 10 being standard sensors (manufactured by SGS Thomson). The outer hexagon holds 36 wider sensors (256 strip, manufactured by Hamamatsu). All the 48 sensor modules of L00 are made of crystallographic $<\mathbf{1 0 0}>$ silicon: 
- Two oxygenated narrow sensor modules. (Manufacturer: Micron)

- Ten standard narrow sensor modules. (Manufacturer: SGS Thomson)

- Thirty-six wide sensor modules. (Manufacturer: Hamamatsu)

The sensors are mounted on a carbon fiber support structure with integrated cooling. In order to assure radiation hardness, the silicon sensors need to be actively cooled. This is achieved by embedded cooling tubes in the carbon fiber structure. The tubes run underneath the entire length of the sensors, cooling them to a temperature below $-5^{\circ} \mathrm{C}$. The 12 inner sensor modules are placed along the beam-line for a total length of $94 \mathrm{~cm}$. The adjacent sensors are ganged together and readout over low-mass, fine-pitched cables. These cables allow the readout electronics to be located outside the tracking volume minimizing multiple-scattering from inactive material. The total number of channels readout in L00 is 13,824. Commissioning was completed May 2002. Greater than $95 \%$ of L00 has been consistently yielding physics quality data since that date. The motivation, design, and construction of $\mathrm{LOO}$ are described in greater detail elsewhere [73].

\subsubsection{SVX-LO}

SVX-II is the core of the silicon detector and it is the only component used in the hardware trigger for events with displaced vertexes [64]. The SVX-II detector has 5 silicon layers (Fig. 4.6) of double-sided sensors located at radii from $2.5 \mathrm{~cm}$ to $10.6 \mathrm{~cm}$. The strip pitch varies between $60 \mu \mathrm{m}$ and $140 \mu \mathrm{m}$, depending on the layer radius.

Optimization of both vertex resolution and pattern recognition considerations lead to layers SVX-LO, SVX-L1 and SVX-L3 to have a "90" stereo" design. The sensors of this three layers were manufactured by Hamamatsu of crystallographic $<111>$ silicon. The $90^{\circ}$ stereo sensors have strips running lengthwise on the sensor's $p-n$ junction side to measure the $r-\phi$ position of the particle, and strips running laterally on the ohmic contact side ( $n$-side) to measure the $r-z$ position. Both sets of strips are read out from the end of the sensors. Hamamatsu sensors can be biased up to $170 \mathrm{~V}$. The remaining two layers, SVX-L2 and SVX-L4, are Micron sensors with $1.2^{\circ}$ angle between the $p$ and $n$ stripes and a maximum bias voltage of $70 \mathrm{~V}$.

From the radiation damage viewpoint, the most critical part of the design of SVX-II is its innermost layer, SVX-LO. SVX-LO is located at a radius of $2.5 \mathrm{~cm}$ and is the closest layer to the interaction region, after L00. SVX-LO is made up of 72 electronic and mechanical independent double-sided modules. The design and characteristics of SVX-LO are described in greater detail elsewhere [72]. 


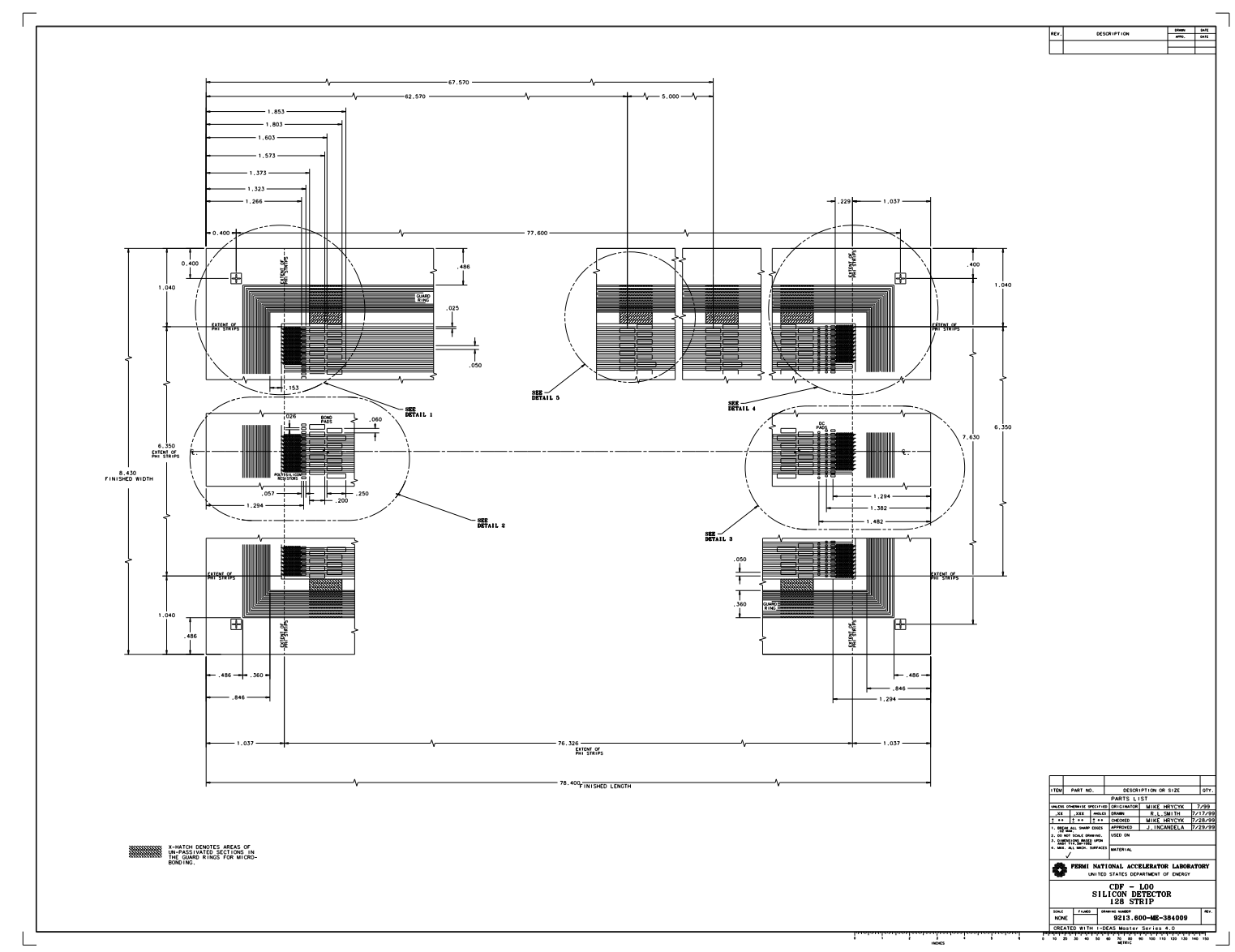

Figure 4.5: Detail of strips and biasing polysilicon resistors (black blocks) for a L00 SGS Thomson sensor.

Table 4.1: Naming convention and detector properties for Layer 00 and the five layers of SVX II

\begin{tabular}{lllll}
\hline Layer & Sensor manufacturer & Strip orientation & Radius $(\mathrm{cm})$ & Max. bias (V) \\
\hline Layer 00 & SGS-Thompson & axial & 1.35 & 500 \\
Layer 00 & Hamamatsu & axial & 1.62 & 500 \\
SVX L0 & Hamamatsu & axial/90 & 2.54 & 160 \\
SVX L1 & Hamamatsu & axial/90 & 4.12 & 160 \\
SVX L2 & Micron & axial/1.2 & 6.52 & 60 \\
SVX L3 & Hamamatsu & axial/90 & 8.22 & 160 \\
SVX L4 & Micron & axial/-1.2 & 10.10 & 60 \\
\hline
\end{tabular}



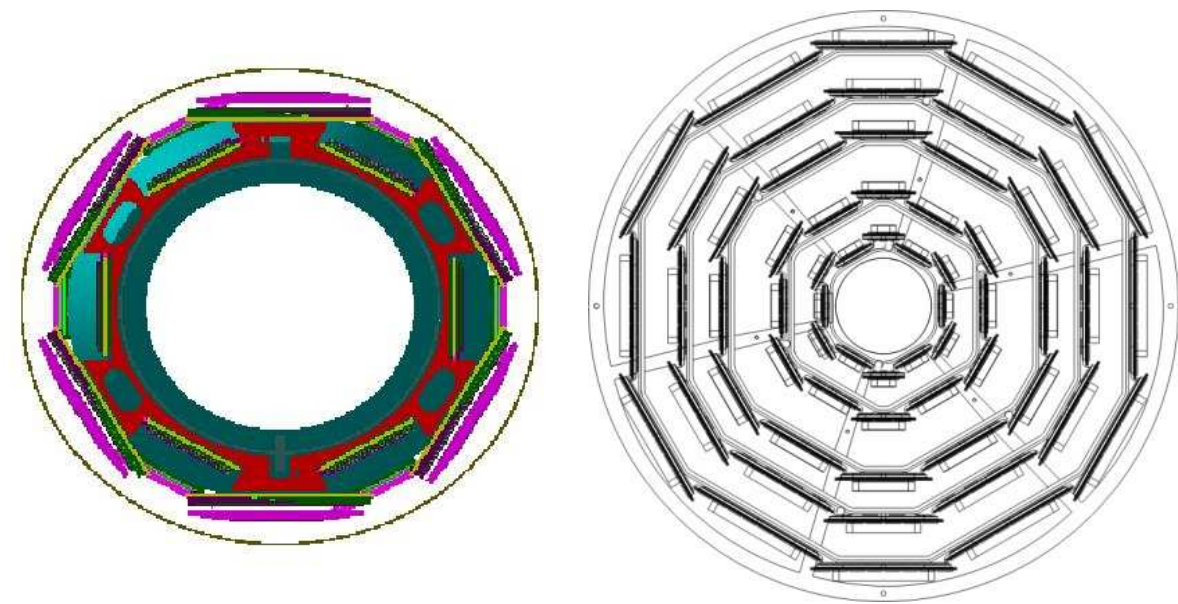

Figure 4.6: Transverse section of L00 (left), and SVX-II (right) whose innermost layer is SVXLO.
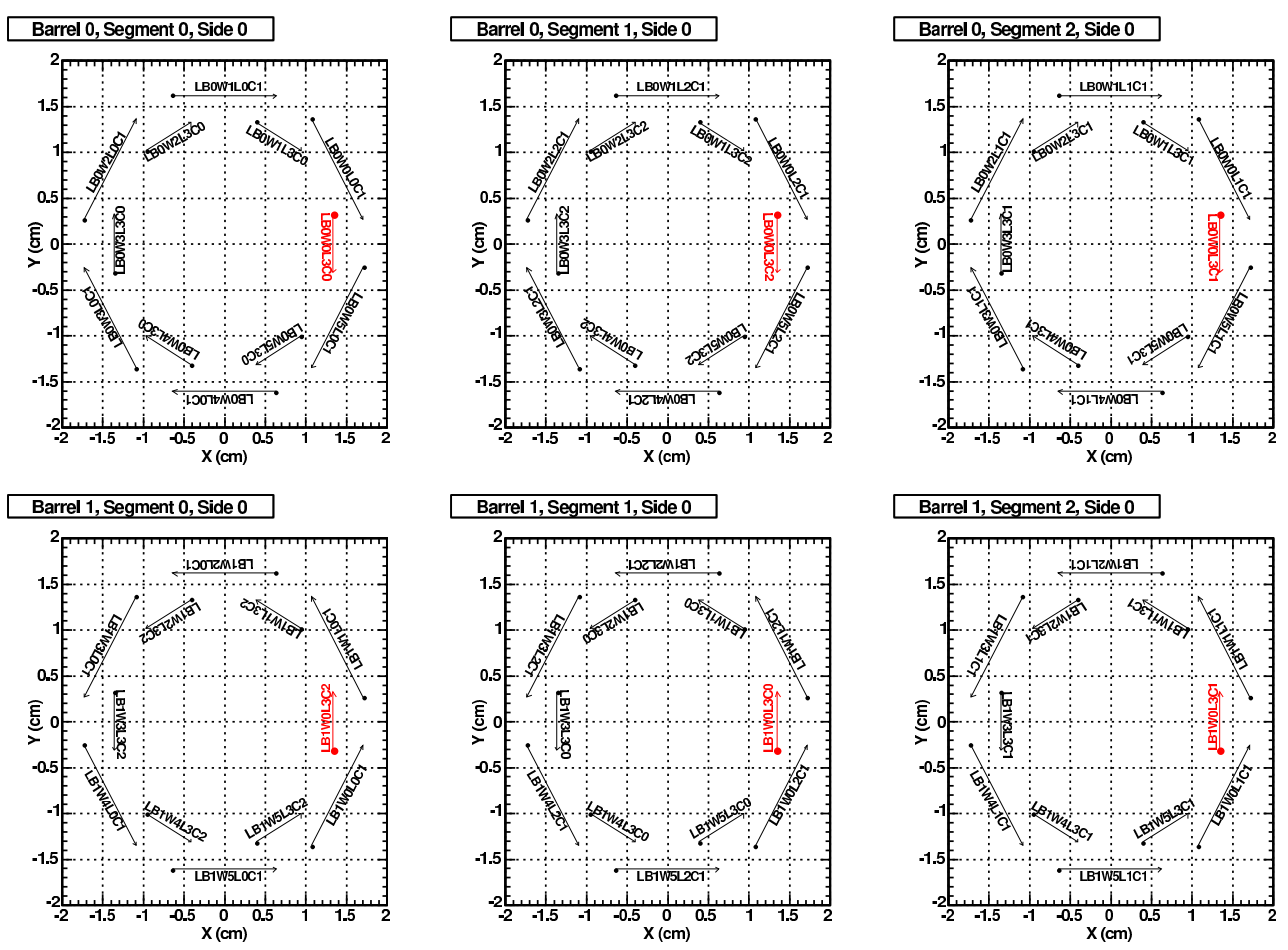

Figure 4.7: Transverse sections of L00 and coordinates of its sensors. 


\subsection{Radiation enviroment}

The measure of the spatial distribution of ionizing radiation and low energy neutrons $\left(E_{n}<\right.$ $200 \mathrm{keV}$ ) inside the tracking volume of the collider detector at Fermilab (CDF) was made using approximately 1000 thermal luminiscent dosimeters (TLD) ([96]). By using data from multiple exposures, the radiation field can be separated into components from beam losses and collisions. The dependence of the radiation field along the dominating component (i.e. collisions) over the CDF volume is radial and proportional to radius $r$ with the power law $r^{-\alpha}$, with $\alpha=1.5$ (Ref. [78], Fig. 4.8).

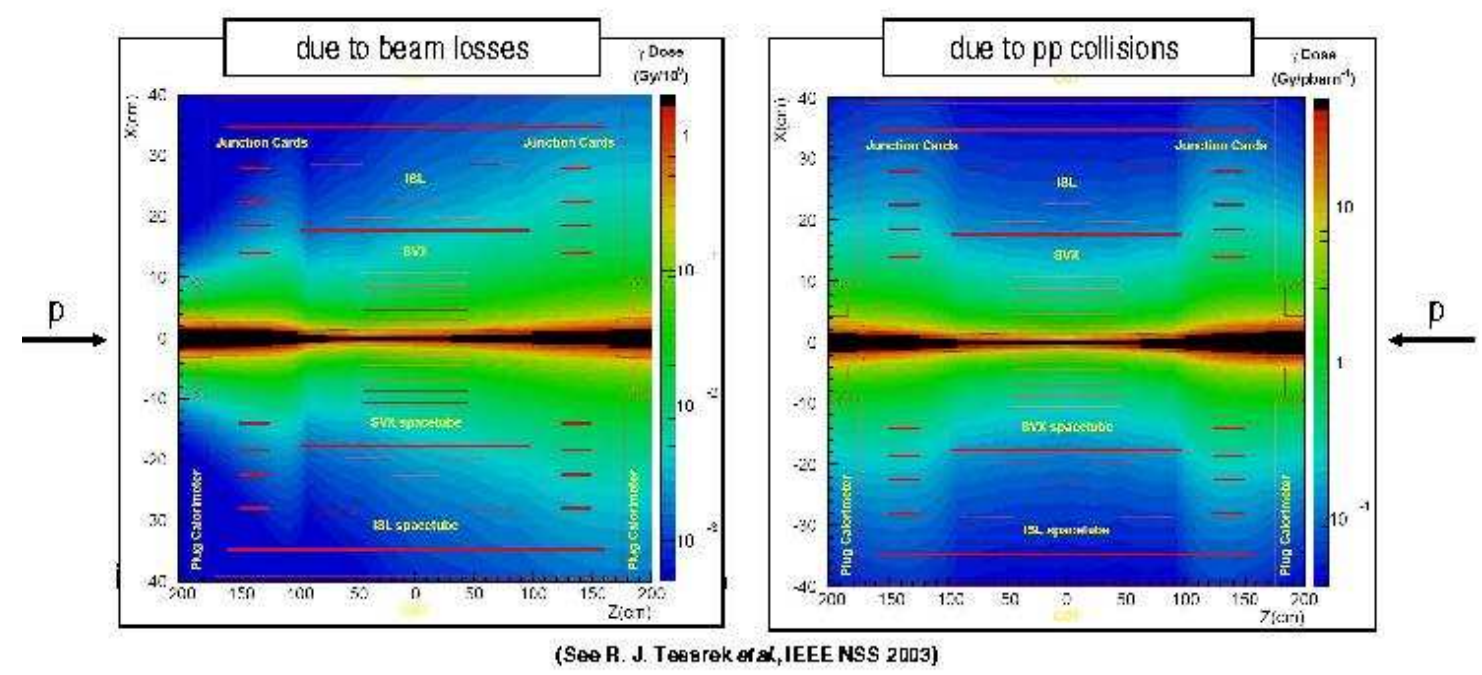

Figure 4.8: Radiation field of CDF. Component of beam losses (left) and collisions.

\subsection{Mitigation of the Radiation Effects}

The efforts to enhance the longevity of the CDF silicon detectors include measures to mitigate the effects of radiation damage and close monitoring of changes of the detector parameters under irradiation. Radiation damage is mitigated by reducing the number of thermo-cycles of the system to a minimum and operating the silicon detectors at a lower temperature. The detector volume was thermally isolated in October 2004, and the detector temperature was lowered from -6 to $-10{ }^{\circ} \mathrm{C}$ in early 2005 .

A measure of the usable lifetime of the CDF silicon detectors is the lowest possible signalto-noise ratio $(S / N)$ acceptable for the SVT and for the ability to perform efficient tagging 
of b quark jets. Based on Monte Carlo simulations from CDF Run I, the lowest possible value is $S / N \approx 6$ [66]. Under irradiation, the noise level increases, and the charge collection efficiency decreases. The signal-to-noise ratio is determined by three factors: the noise level of readout electronics, the noise level of sensors and the charge collection efficiency of the sensors. Therefore, in agreement with detailed studies [75] [76] the noise level of readout electronics is not expected to be the limiting factor for the silicon lifespan.

The sensistivity of the readout electronics to radiation has been measured [90] to be less than that of the sensors not limiting the detector ultimate performance.

With regard to the second of the factors, the dominant source of noise is the shot noise of the silicon sensors. The shot noise is increased by crystal defects in the sensors due to radiation damage. For SVX II, the shot noise can be parametrized as

$$
Q=900 e \times \sqrt{I_{\text {leak }}},
$$

where $e$ is the electron charge and $I_{\text {leak }}$ is the leakage current in $\mu \mathrm{A}$ [66]. Changes in the leakage current can be approximated by changes in the bias current $I_{\text {bias }}$, assuming that all other contributions to the bias current are constant:

$$
\Delta I_{\text {leak }} \approx \Delta I_{\text {bias }}
$$

The change in bias current is related to the fluence $\Phi$ by $\Delta I_{\text {bias }}=\alpha \Phi V$, with the damage constant $\alpha$ and the known detector volume $V$. An effective damage constant of $\alpha_{e f f}=(3.98 \pm 0.15) \times 10^{-17}$ $\mathrm{A} / \mathrm{cm}$ is derived by comparing the radial dependence of $\Delta I_{\text {bias }}$ with the radial dependence of the radiation field. The TLD measurements agree with measurements of $\Delta I_{\text {bias }}$ within $10 \%$ [69]. Hence the fluence can be inferred from bias current measurement. The main systematic uncertainty of this method $(13 \%)$ is due to the imprecise knowledge of the SVXII sensor temperatures since the SVXII modules are only cooled from the modules' end, where the readout electronics are located.

Regarding the third of the factors affecting the signal-to-noise ratio, sufficient charge collection efficiency is achieved by applying a bias voltage between the two sides of a silicon sensor. Under irradiation and annealing, the effective number of charge carriers changes, and the bulk of the sensor undergoes "type inversion" from net $n$-type to $p$-type silicon. The bias voltage required to fully deplete the sensor decreases until type inversion occurs and increases afterwards, eventualy surpassing the maximum safe bias voltage. Breakdown of the coupling capacitors on the SVX II sensors limits the maximum safe bias voltage to approximately $160 \mathrm{~V}$ for the Micron sensors. The LHC-style sensors in Layer 00 can be biased up to $500 \mathrm{~V}$. In Ref. [66], a model of the depletion voltage is presented, and the lifetime of the silicon detectors is predicted, albeit with large uncertainties. 
The harsh radiation environment in which the detectors are operated is responsible of the progressive increment on the bias voltage needed to fully deplete the sensors. Since the accuracy of the whole instrument depends on a correct depletion status of the diodes, a test to monitor such status is needed: the signal-to-bias scan, which will be discussed in chapter 6. The signal-to-bias scan test lies essentially on making use of the detector to collect a set of data each one of them at a different bias voltage, ranging from zero to a nominal depletion voltage. The result of this process is able to show if the sensors are correctly depleted. The scans are performed periodically every 4-6 weeks and are useful both for detecting underdepleted sensors and to derive information about the silicon detector life span. 


\section{Chapter 5}

\section{Leakage Current Analysis}

In order to properly characterize the detector, this chapter will first describe in detail the system that brings the voltage to the sensor and is responsible for the reverse polarization of the strips. This naturaly will bring us the analysis of the bias currents that cross the reverse-biased diodes. Some care will be devoted to the discussion of all series resistances in the circuit since it is necessary to know the actual voltage drop at the sensor, which will become relevant in the study of the depletion voltage in chapter 6 .

\subsection{Bias scheme}

The computation of the series resistance has to take account of the internal resistances of the elements of the circuit (see Fig. 5.1): the CAEN power supply, cabling, junction cards, port cards, hybrids, and bias resistors of the sensors. The following sections show the detailed description of everyone of those circuit elements.

The three basic requirements for the SVX II Power Supply system are to provide all the power for the silicon modules, the SVX3 chip set and the Port Cards (PC); to control and monitor all the relevant output voltages and currents; and to provide failure mode protection for safe power supply and system operation.

The module consists of double-sided AC coupled silicon microstrip detectors, the analog and digital frontend electronics (SVX3 chip set), and a hybrid device. DC high voltage ( 200 $\mathrm{V})$ is needed to bias the silicon detectors, and DC low voltage $(5 \mathrm{~V})$ is needed to power the analog, digital, and I/O electronics. Thus, a Power Supply Module must deliver to the module a number of voltages with different current, stability, and monitoring requirements. The 5 modules in one of the 12 sectors of a barrel share a PortCard (PC) that contains the optical transmitters and distributes power to the modules. 


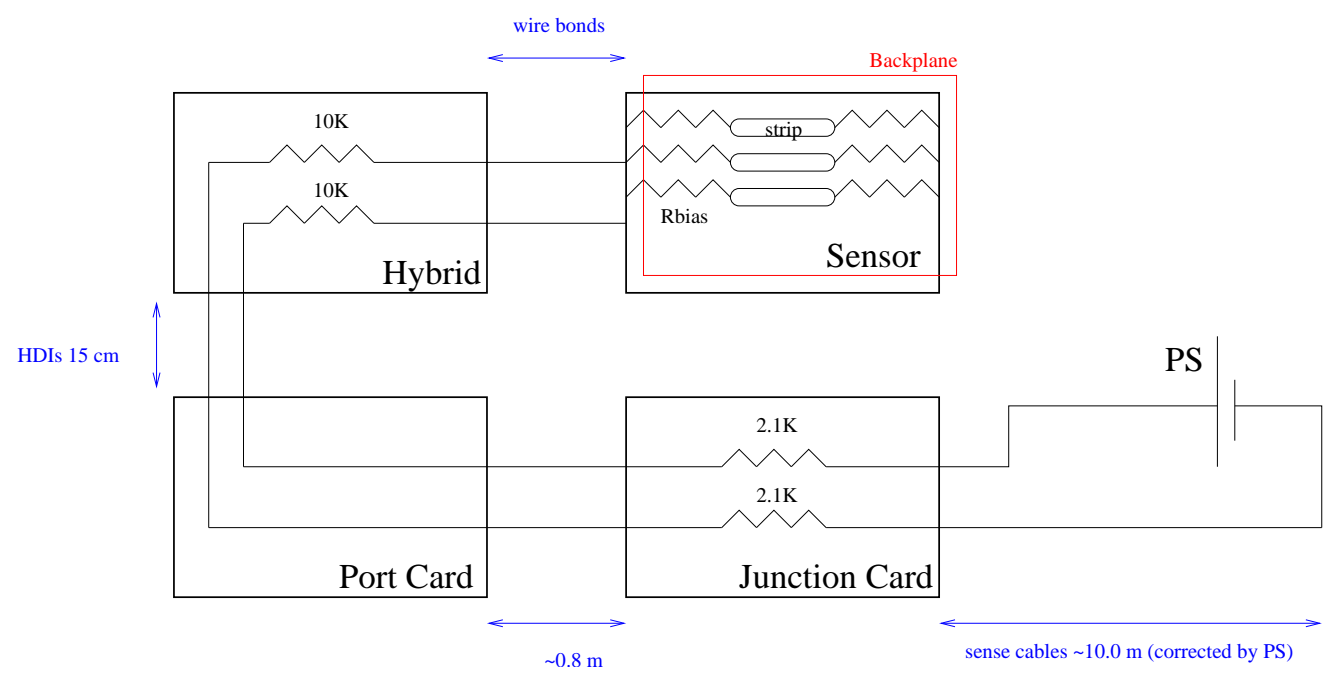

Figure 5.1: Silicon detector bias circuit.

\subsubsection{CAEN PS}

The Model A509H CDF-L00 Power Supply Board has been specially designed for the CDF Silicon Vertex Detector at Fermilab. The board is housed in an 8 TE-wide, 6U-high mechanics to be inserted in the SY527 system mainframe. Its basic function is to deliver a set of floating voltages to drive a detector's sector which consists of a Port Card and four silicon ladders (Layer 0 through Layer 3). Moreover, the board has a monitoring circuit of output voltages, currents and status and associated control and protection circuits. For more details see Ref. [89].

\subsubsection{Cables}

The connection from the PS to the $\mathrm{PC}$ is made through cables that are assembled in bundles consisting of one voltage cable, one sense, one command, one bias voltage cable and 5 optical cables. Power Supplies connect with the detector's Junction Cards, port devices that are located inside the Collision Hall about $10 \mathrm{~m}$ away from the detector volume. The nominal voltage supplied is compensated by the Power Supply with the voltage drop corresponding to the length of the cables.

Data from the detector will travel over optical fibers while control signals, power and bias (high) voltage will travel over conventional copper lines. There are 11 differential control signals per PC, one of which is a $53 \mathrm{MHz}$ clock while the other is a beam crossing clock. Each module will have an independent bias voltage and three power voltages. In addition, the $\mathrm{PC}$ requires 4 separate voltages. 


\subsubsection{Junction cards}

The Junction Card is an interface block located between the Port Card and the Power Supply. The Junction Card collects power, bias voltages and control lines for an entire wedge together in one 90-pin connector. Junction Cards are the last human-reachable points along the power and control cable supply lines. The internal resistance of the Junction Card block is $2.1 \mathrm{~K} \Omega$ (Fig. 5.2).

\subsubsection{Port cards}

The Port Card (PC) is a beryllia multichip module located on the top of the wedge, 14 $\mathrm{cm}$ from the accelerator beam inside the tracking volume. The PC communicates with the SVX3 chips through high density interconnect cables (HDIs). The main functions of the PC are control, configuration and readout of the SVX3 chips, and to locally regulate the analog power supplies [88]. The analog section of the PC (Figs. 5.3, 5.4) is formed by a voltage regulator and high voltage bias for the sensors. The voltage regulators are assembled on the PC itself to keep them close to the SVX3 hybrids. The bias is delivered directly to the hybrids where they are low-pass filtered and connected to the sensor. The internal resistance of the Port Card for the power line is zero.

\subsubsection{Hybrids}

The microstrip sensors are read out through Application Specific Integrated Circuits (ASIC) readout chips, the SVX3, assembled in hybrids bonded to both sides of the sensors. The pre-amplifier inputs of the SVX3 chips are wire bonded directly to the detector strips. The SVXII readout hybrid processes the detector signals into a format suitable for the port card. Associated with the readout hybrid is a cable that connects the power/signal functions from the hybrid to the port card. The hybrid consists of 6 interconnected gold ink layers, separated by a dielectric, on top of a ceramic substrate. This is a standard ink for applications that require wire bonding. Additionally, there are palladium-gold ink pads on the top layer to permit soldering of surface mount components. The internal resistance of the hybrid block is $10 \mathrm{~K} \Omega$.

\subsubsection{Bias resistors on the detector}

The bias voltage is applied to the strips through polysilicon bias resistors. Tables 5.1 and 5.2 show the bias resistors values corresponding to the different sensors of LOO and SVXII, respectively. 


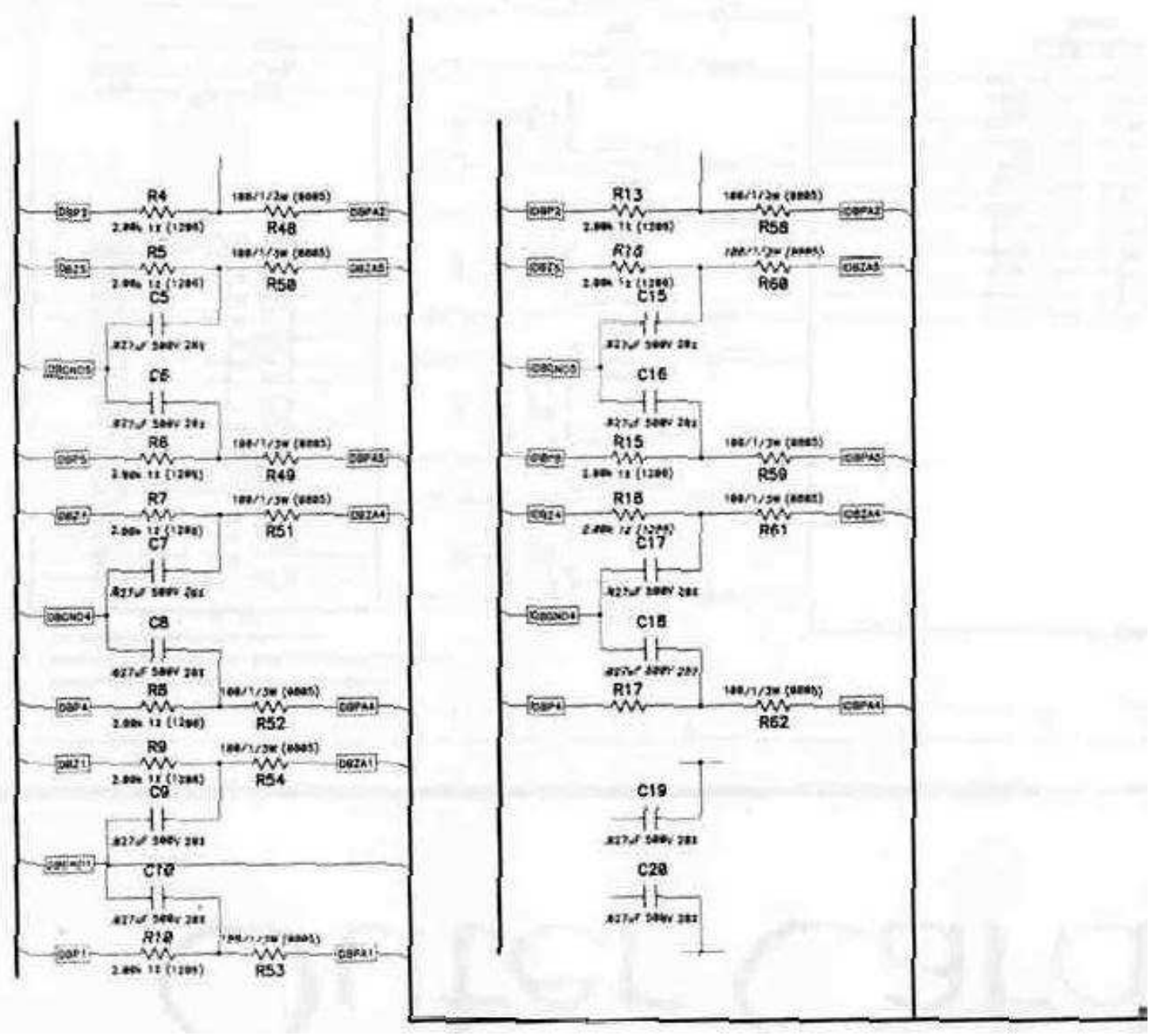

Figure 5.2: Outer Junction Card circuit. 


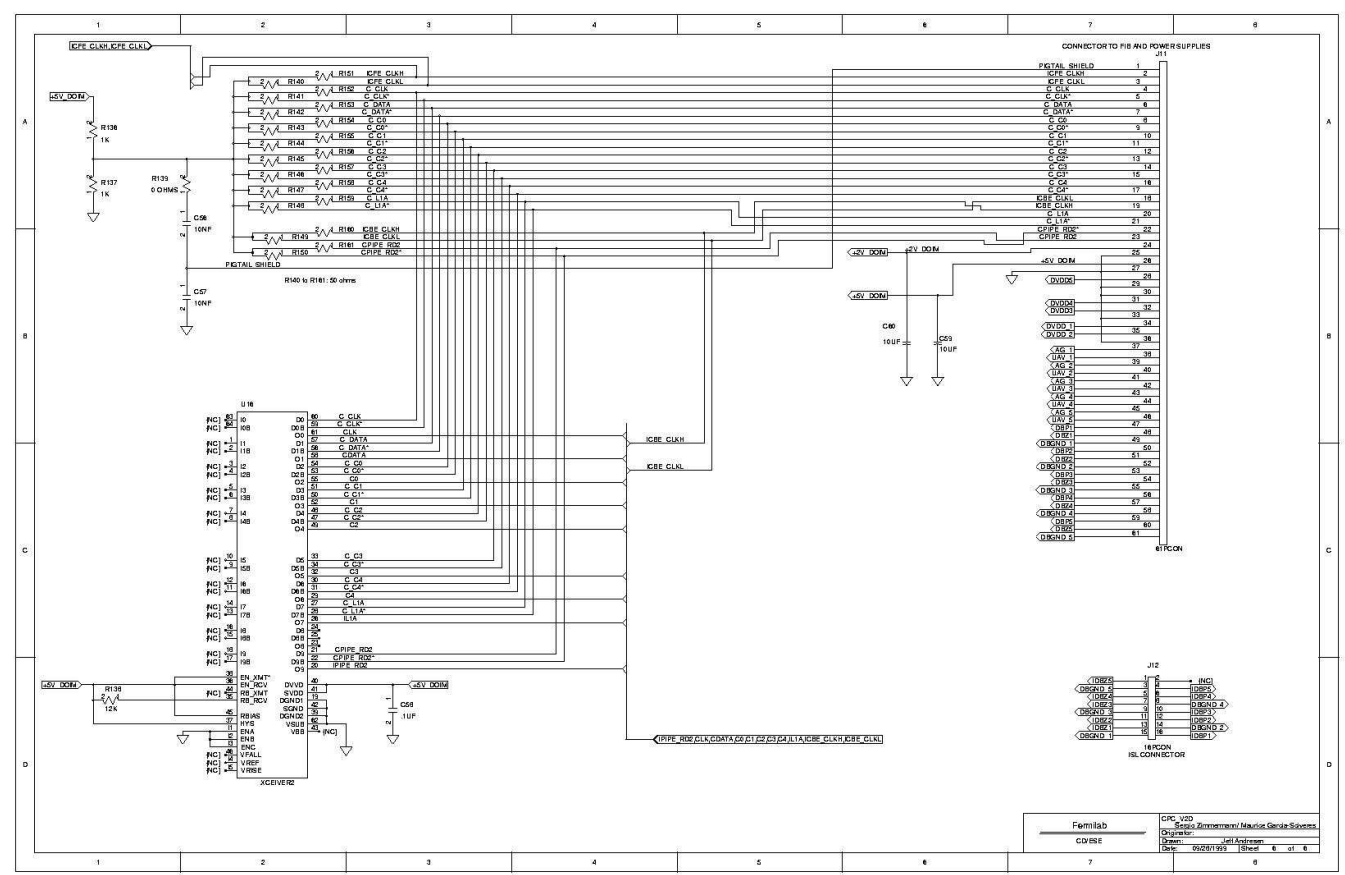

Figure 5.3: Port Card circuit.

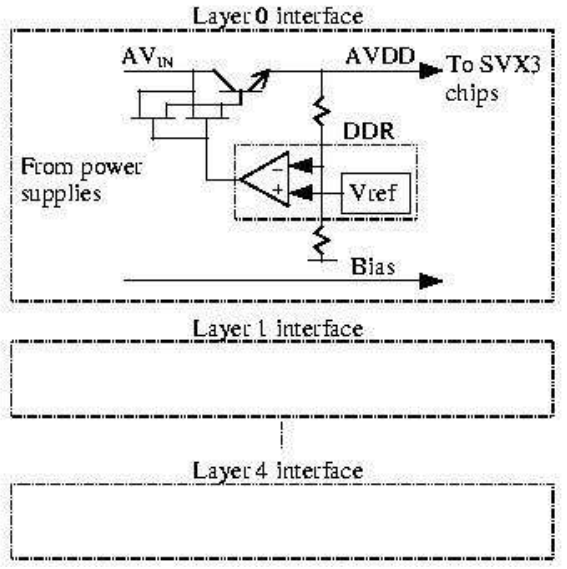

Figure 5.4: Port Card analog section. 
Table 5.1: Technical parameters of L00 sensors. Last row contains the polysilicon biasing resistor values needed for the total resistance computation.

\begin{tabular}{|c|c|c|}
\hline L00 & $\begin{array}{l}\text { Hamamatsu } \\
\text { (wides) }\end{array}$ & $\begin{array}{l}\text { SGS-Thomson } \\
\text { (narrows) }\end{array}$ \\
\hline Type & $\begin{array}{l}\text { single-sided, } \\
\mathrm{p} \text { in } \mathrm{n}, \mathrm{AC} \\
\text { coupled, } \\
\text { polysilicon } \\
\text { bias }\end{array}$ & $\begin{array}{l}\text { single-sided, } \\
\mathrm{p} \text { in } \mathrm{n}, \mathrm{AC} \\
\text { coupled, } \\
\text { polysilicon } \\
\text { bias }\end{array}$ \\
\hline Thickness & $\begin{array}{l}300 \pm 20 \mathrm{mi}^{-} \\
\text {crons }\end{array}$ & $\begin{array}{l}300 \pm 20 \text { mi- } \\
\text { crons }\end{array}$ \\
\hline Warp & $<80$ microns & $<50$ microns \\
\hline Readout Pitch & 50 microns & 50 microns \\
\hline Implant Pitch & 25 microns & 25 microns \\
\hline Implant Width & 8 microns & 8 microns \\
\hline Al Strip Width & $7-8$ microns & $7-8$ microns \\
\hline $\begin{array}{l}\text { AC Coupling } \\
\text { Capacitance }\end{array}$ & $>10 \mathrm{pf} / \mathrm{cm}$ & $>100 \mathrm{pf} / \mathrm{cm}$ \\
\hline $\begin{array}{l}\text { Interstrip } \mathrm{Ca}- \\
\text { pacitance }\end{array}$ & $<1.2 \mathrm{pf} / \mathrm{cm}$ & $<1.2 \mathrm{pf} / \mathrm{cm}$ \\
\hline $\begin{array}{l}\text { Interstrip Resis- } \\
\text { tance }\end{array}$ & $>2 \mathrm{G} \Omega$ & $>2 \mathrm{G} \Omega$ \\
\hline $\begin{array}{l}\text { Breakdown/Micr } \\
\text { discharge Volt- } \\
\text { age }\end{array}$ & $>350 \mathrm{~V}$ & $>600 \mathrm{~V}$ \\
\hline $\begin{array}{l}\text { Polysilicon re- } \\
\text { sistor }\end{array}$ & $4.5 \pm 0.5 \mathrm{M} \Omega$ & $4.5 \pm 0.5 \mathrm{M} \Omega$ \\
\hline
\end{tabular}


Table 5.2: Technical parameters of SVXII sensors. Eighth row contains the polysilicon biasing resistor values needed for the total resistance computation.

\begin{tabular}{|c|c|c|}
\hline SVXII & Hamamatsu & Micron \\
\hline $\begin{array}{lr}\mathrm{I}_{\text {leakage }} & \text { current } \\
\text { from } & \text { bulk, } \\
\text { surface, } & \text { and } \\
\text { edge } & \end{array}$ & $\begin{array}{l}<50 \mathrm{nA} / \mathrm{cm} 2 \\
100 \mathrm{~V}, 20 \mathrm{C}\end{array}$ & $\begin{array}{l}<100 \mathrm{nA} / \mathrm{cm} 2 \text { at } \\
80 \mathrm{~V}, 20 \mathrm{C}\end{array}$ \\
\hline $\begin{array}{l}\mathrm{dI}_{\text {leakage/dV }} \\
\text { change in } \\
\text { leakage current }\end{array}$ & $<0.12 \mathrm{nA} / \mathrm{V} / \mathrm{cm} 2$ & - \\
\hline $\begin{array}{l}\mathrm{V}_{\text {depletion }} \text { deple- } \\
\text { tion voltage }\end{array}$ & $\begin{array}{l}45 \mathrm{~V}< \\
V_{d e p}<80 \mathrm{~V}\end{array}$ & - \\
\hline $\begin{array}{l}\rho \text { bulk resistiv- } \\
\text { ity }\end{array}$ & - & $\mathrm{Apx}=3 \mathrm{~K} \Omega \mathrm{cm}$ \\
\hline $\begin{array}{l}\mathrm{V}_{\text {junction }_{b} \text { reak }} \\
\text { junction break- } \\
\text { down with open } \\
\text { readout }\end{array}$ & $>200 \mathrm{~V}$ & $>150 \mathrm{~V}$ \\
\hline $\begin{array}{l}\mathrm{V}_{\text {microdischarge }} \\
\text { onset voltage of } \\
\text { micro-discharge } \\
\text { ( } \mathrm{n} \text { and } \mathrm{p} \text { side) }\end{array}$ & $>150 \mathrm{~V}$ & $>150 \mathrm{~V}$ \\
\hline $\begin{array}{l}\mathrm{R}_{\text {interstrip }} \text { inter- } \\
\text { strip resistance }\end{array}$ & $>2 \mathrm{G} \Omega$ & $>2 \mathrm{G} \Omega$ \\
\hline $\begin{array}{ll}\mathrm{R}_{\text {bias }} & \text { polysil- } \\
\text { icon } & \text { resistor } \\
\text { value, variation } \\
\text { p- and } \mathrm{n} \text {-side }\end{array}$ & $\begin{array}{l}2.5+1.0 \quad \mathrm{M} \Omega \\
3.5+1.0 \mathrm{M} \Omega\end{array}$ & $\begin{array}{l}2.5+0.5 \mathrm{M} \Omega, \\
2.5+0.5 \mathrm{M} \Omega\end{array}$ \\
\hline $\begin{array}{l}\mathrm{R}_{\text {implant }} \text { resistiv- } \\
\text { ity of implant } \\
\text { strip }\end{array}$ & $<100 \mathrm{~K} \Omega / \mathrm{cm}$ & - \\
\hline $\begin{array}{l}\mathrm{R}_{A l} \text { resistivity } \\
\text { of } \mathrm{Al} \text { layers }\end{array}$ & $<25 \Omega / \mathrm{cm}$ & $<30 \Omega / \mathrm{cm}$ \\
\hline $\begin{array}{l}\mathrm{C}_{C} \text { capacitance } \\
\text { of coupling ca- } \\
\text { pacitor }\end{array}$ & $>10 \mathrm{pF} / \mathrm{cm}$ & $>12 \mathrm{pF} / \mathrm{cm}$ \\
\hline
\end{tabular}




\subsubsection{Total resistance computation}

The purpose of this section is to evaluate the series resistance of the loop of the bias circuit (Fig. 5.1). The desired total resistance $R_{\text {total }}$ would be the sum (series) of the internal resistances of the loop: $R_{\text {loop }}$ (corresponding to blocks "Junction Card", "Hybrid", and "Port Card"), plus the internal resistance of the sensor: $R_{\text {sensor }}$ (made up of $n$ parallel biasing resistances $R_{\text {bias }}$ ).

The general formula for $R_{\text {sensor }}$ (Eq. 5.3) includes the value of the biasing resistance $\left(R_{\text {bias }}\right)$, the multiplicity of biasing resistance for each strip (nbias; whose value is $\mathbf{2}$ for $\mathbf{L O 0}$ since each strip is biased in its two extrems, and 1 for SVX with a single biasing resistance per strip), the number of sensors per channel (nchannels) and the number of strips per sensor (nstrips). This values are summarized in Tables 5.3, 5.1 and 5.2. In the case of the double-sided sensors of SVX the $R_{\text {sensor }}$ is the sum in series of both the phi and $z$ sides (Eq. 5.5).

$$
\begin{gathered}
R_{\text {total }}=R_{\text {loop }}+R_{\text {sensor }} \\
R_{\text {loop }}=24.2 \mathrm{~K} \Omega \\
R_{\text {sensor }}=\left(R_{\text {bias }} /(\text { nbias }) *(\text { nchannels }) *(\text { nstrips })\right) \\
R_{\text {sensorL } 00}=\left(R_{\text {bias }} /(2) *(\text { nchannels }) *(\text { nstrips })\right) \\
R_{\text {sensorSVX }}=R_{\text {sensor }, \phi}+R_{\text {sensor }, z}= \\
=\left(R_{\text {bias }, \phi} /(1) *(\text { nchannels }, \phi) *(\text { nstrips }, \phi)\right)+\left(R_{\text {bias }, z} /(1) *(\text { nchannels }, z) *(\text { nstrips }, z)\right)
\end{gathered}
$$

Table 5.3 summarizes the electronic parameters and the computation of the total resistance of the circuit for the different sensors of LOO and SVX. Relating for example the LOO internal resistance results with the corresponding LOO bias current data (Fig. 5.5) it is possible to obtain the voltage drop due to the bias voltage circuit. This drop ranges between 0.5 and 7.5 volts, depending on the sensor type.

\subsection{Radiation Damage Quantification}

Bias currents in a silicon detector can be used to quantify the radiation damage in the detector itself [77]. The bias current is expected to increase with received radiation dose because of the radiation-created defects in the silicon lattice. An increase in bias current eventually causes an increase in noise, so reducing the signal-to-noise ratio. Thus, measuring the bias current is an important way to estimate the lifetime of a silicon detector. 
Table 5.3: Summary of technical parameters of L00 and SVXII sensors needed for the total resistance computation.

\begin{tabular}{|c|c|c|c|c|c|c|c|}
\hline Layer & Sensor & $\begin{array}{l}\text { Bias resis- } \\
\text { tor }\end{array}$ & Num. strips & $\begin{array}{l}\text { PS } \\
\text { chan- } \\
\text { nels }\end{array}$ & $\begin{array}{l}\mathbf{R}_{\text {loop }} \\
{[\mathbf{K} \Omega]}\end{array}$ & $\begin{array}{l}\mathbf{R}_{\text {sensor }} \\
{[\mathrm{K} \Omega]}\end{array}$ & $\begin{array}{l}\mathbf{R}_{\text {total }} \\
{[\mathrm{K} \Omega]}\end{array}$ \\
\hline L00 & Hamamatsu & $\begin{array}{l}4.5 \mathrm{M} \Omega \text { (at } \\
\text { both sides of } \\
\text { strip) }\end{array}$ & 128 & 3 & 24.2 & 5.86 & 30.06 \\
\hline LO0 & $\begin{array}{l}\text { SGS- } \\
\text { Thomson }\end{array}$ & $\begin{array}{l}4.5 \mathrm{M} \Omega \text { (at } \\
\text { both sides of } \\
\text { strip) }\end{array}$ & 128 & 2 & 24.2 & 8.8 & 33.0 \\
\hline $\mathrm{LO0}$ & Micron & $\begin{array}{l}4.5 \mathrm{M} \Omega \text { (at } \\
\text { both sides of } \\
\text { strip) }\end{array}$ & 128 & 1 & 24.2 & 17.57 & 41.77 \\
\hline
\end{tabular}

\begin{tabular}{||l||l|l|l|l|l|l|l||}
\hline \hline & $\begin{array}{l}\text { phi-side/z- } \\
\text { side }\end{array}$ & $\begin{array}{l}\text { phi-side/z- } \\
\text { side }\end{array}$ & & & & \\
\hline SVX-L0 & Hamamatsu & $\begin{array}{l}2.5 / 3.5 \mathrm{M} \Omega \\
\text { (at one side } \\
\text { of strip) }\end{array}$ & $256 / 512$ & 2 & 24.2 & 8.3 & $\mathbf{3 2 . 5}$ \\
\hline SVX-L1 & Hamamatsu & $\begin{array}{l}2.5 / 3.5 \mathrm{M} \Omega \\
\text { (at one side } \\
\text { of strip) }\end{array}$ & $384 / 576$ & 2 & 24.2 & 6.25 & $\mathbf{3 0 . 4 5}$ \\
\hline SVX-L2 & Micron & $\begin{array}{l}2.5 / 2.5 \mathrm{M} \Omega \\
\text { (at one side } \\
\text { of strip) }\end{array}$ & $640 / 640$ & 2 & 24.2 & 3.85 & $\mathbf{2 8 . 0 5}$ \\
\hline SVX-L3 & Hamamatsu & $\begin{array}{l}2.5 / 3.5 \mathrm{M} \Omega \\
\text { (at one side } \\
\text { of strip) }\end{array}$ & $768 / 512$ & 2 & 24.2 & 5 & $\mathbf{2 9 . 2}$ \\
\hline SVX-L4 & $\begin{array}{l}2.5 / 2.5 \mathrm{M} \Omega \\
\text { (at one side } \\
\text { of strip) }\end{array}$ & $896 / 896$ & 2 & 24.2 & 2.8 & $\mathbf{2 7 . 0}$ \\
\hline
\end{tabular}


An analysis of bias currents was made by P. Dong et al. [78] for the SVX II layers. With the idea of completing these studies to the rest of the CDF silicon detectors, this section is dedicated to the analysis of the bias currents in $\mathrm{LOO}$.

The bias current in a silicon detector can be expressed as the sum of four components: a bulk current, due to processes in the volume of the silicon detector; a surface current, due to processes at the interface with the oxide layer; an edge current, due to processes resulting from the cutting of the crystal; and a stray current, which accounts for any leakage in cables and electronics:

$$
I_{\text {bias }}=I_{\text {bulk }}+I_{\text {surface }}+I_{\text {edge }}+I_{\text {stray }}
$$

Similarly to the SVX II layers case [78], $I_{\text {stray }}$ should remain constant for L00, since the cables and electronics do not change. Edge currents are expected to be small and roughly constant, since the region in which they occur is very small; and surface currents are expected to saturate early in the detector's life. Thus, once the sensors have been sufficiently irradiated, the increase in bias current should be dominated by bulk current. Bulk current increases linearly with the delivered radiation:

$$
\Delta I_{\text {bulk }}=\alpha_{\text {damage }} \Phi V
$$

where $V$ is the volume, $\Phi$ is the particle fluence, and $\alpha_{\text {damage }}$ is a constant. The effective fluence $\Phi$ can be obtained from equation 5.8. However, two more variables are required to be known: the temperature of the silicon and the damage coefficient $\alpha_{\text {damage }}$. Since reverse bias currents are the result of electrons moving from the valence band to the conduction band, higher temperatures will result in more thermal excitations of electrons yielding that the leakage current scales exponentially with temperature. Fortunately for LO0 we can have an accurate measure of the temperature of its sensors since they are actively cooled at an operation value of $-10^{\circ} \mathrm{C}$.

\subsection{Bias current data}

The bias currents for each power supply cannel (usually one half-module) of silicon are measured by the Caen power supplies and written to a text file every nine seconds if they differ from the previous measurement. For LO0 there are recorded data up to $7000 \mathrm{pb}^{-1}$. An example of the general linear relation between LOO bias current and luminosity -an outcome of the luminosity fluence proportionality- is shown in Fig 5.5. The current does 
not increase linearly with time. This original data of bias current versus luminosity were corrected back to a temperature of $20 \mathrm{C}$.
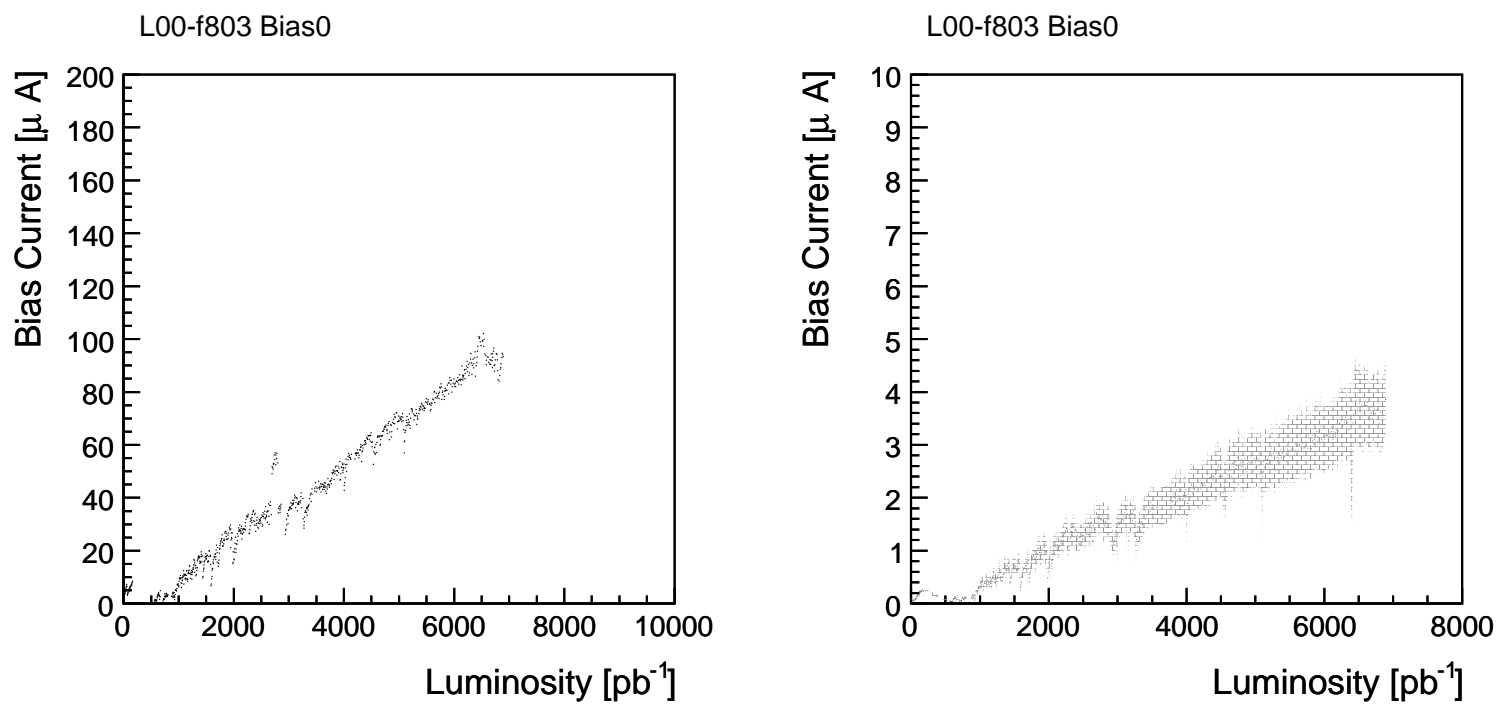

Figure 5.5: Bias current data of L00 sensor f803. Original (left) and temperature corrected at $20^{\circ} \mathrm{C}$ (right).

Apart from the bias current, the other set of original data needed for this analysis is the distribution of the beam position with luminosity, shown in Fig. 5.6. Since the beam position changes with time, this set of data is required to compute the real distance of the beam vertex to each one of the LOO sensors along the luminosity values. This calculation is critical since $\mathrm{LOO}$ is the closest sensor device to the beam and the radiation field falls off radially according to a power law [79] [80].

\subsection{Analysis of the bias currents}

The analysis of Dong et al. [78] used bias currents data which covered the period from May 22 to July 14, 2004, which corresponds to $95 \mathrm{pb}^{-1}$ (Tevatron stores from 3528 to 3653). Original LOO bias current data (Fig. 5.8) were corrected by temperature in the following way. Since the refrigeration channels are in thermal contact with the mechanical framework of the sensors, the temperature of the sensors (i. e. the closest ones to the channel) is considered to be the same temperature measured in the refrigerant. Each refrigeration line is omnitored bt two temperature sensors, one at the entrance and another at the exit of the 

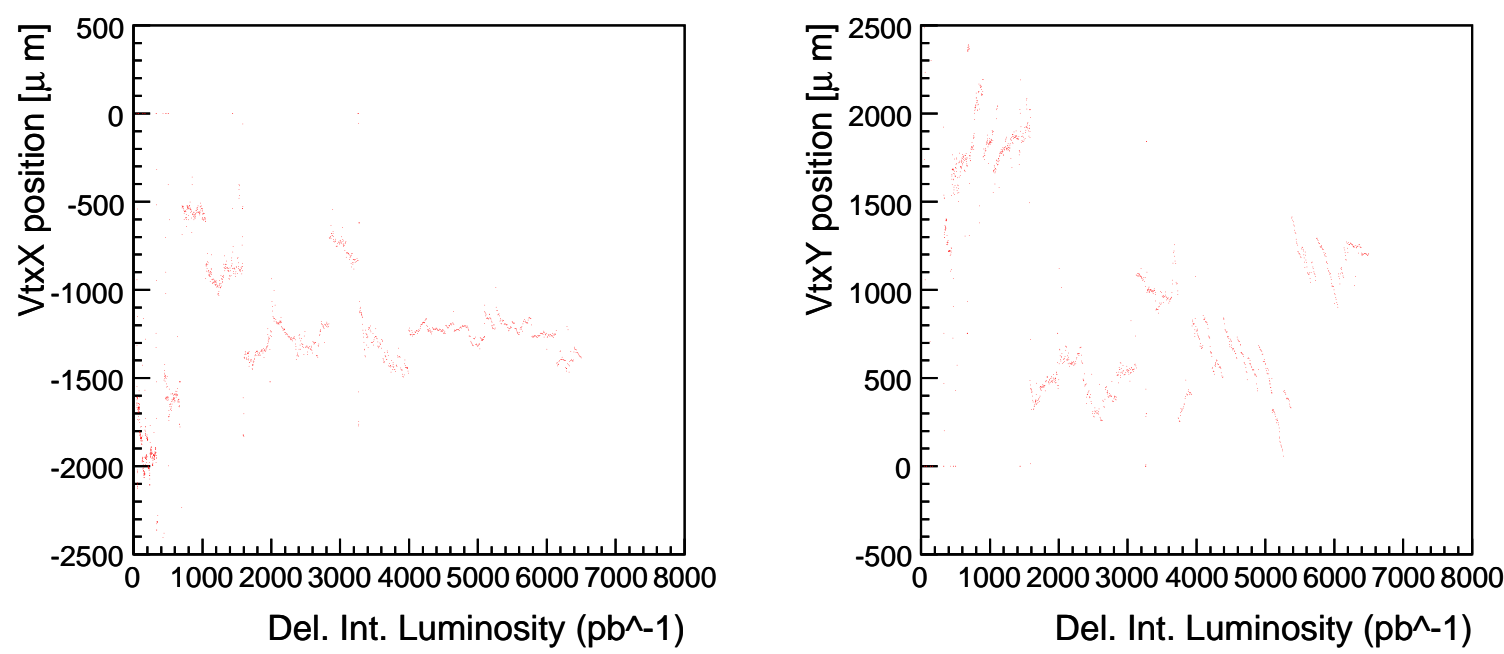

Figure 5.6: Vertex position (x-coordinate, on the left; y-coordinate, on the right) vs. luminosity.

refrigerant flux. The temperature (in a particular instant, being recorded daily) is defined as the average of the temperatures of those two sensors. Entering this temperature in Eq. C.26 (Appendix C) together with a reference temperature $\left(20^{\circ} \mathrm{C}\right)$ it is possible to extract a correction factor applicable to the original bias current data.

In order to make comparable our LO0 analysis to Dong's SVX II analysis we defined fitting-windows of width $95 \mathrm{pb}^{-1}$ along the $\mathrm{x}$-axis of luminosity. Then, the slopes were superimposed over their corresponding windows, so having a plot of the slopes versus luminosity as shown in the plot on the left in Fig. 5.7. Finally, the $x$-axis of luminosity was replaced by the sensor's corresponding distance to the beam (Fig. 5.7, right plot) and the points fitted with a linear function. The slope of the linear fit in the right plot of Figure 5.7 seems to be high. However, the computation appears to be distorted by the point located at $1.3 \mathrm{~cm}$. This slope point corresponds to the earliest luminosities where the bias currents had a pattern of high-rising slopes and followed by sudden decreases to zero when the sensor was turned off. This behavior can be observed in a zoom of the bias currents plot in the range from 0 to $1000 \mathrm{pb}^{-1}$ (Fig. 5.9). After this considerations, if the points of lowest luminosities were removed from the computation of the slopes, the result would be an almost flat distribution of slopes versus distance. This means that the slope of the bias current of any L00 sensor would not be affected by the beam position (in the measured range) and hence this kind of correction is neglected. 
L00-f820 Bias0 Linear fit slope $=0.000861$

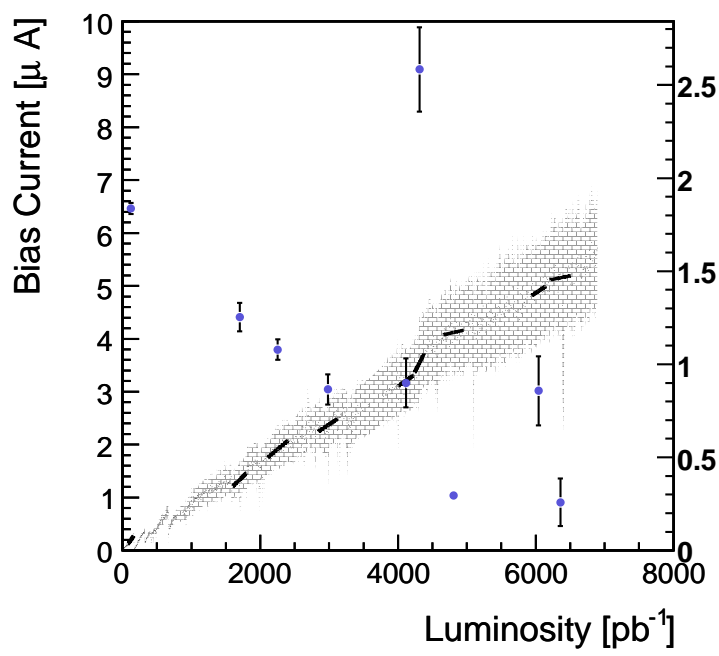

Sensor: 1820

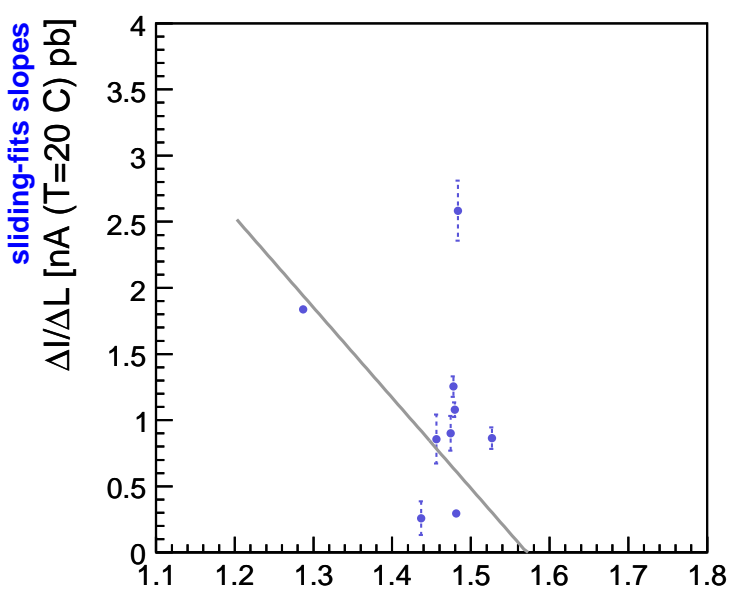

Distance from sensor f820 to vertex [cm]

Figure 5.7: Example of sliding-fits slopes for sensor f820 (left) and sliding-fits slopes versus distance from the vertex to the sensor (right).

\subsection{Total Bias Current}

Figure 5.8 shows a general picture of the total bias current measured for the two classes of L00 sensors: narrows and wides. The vertical axis is the sum of the currents over the number of sensors. The plot on the left (narrows) covers the sum of currents read by the 12 narrow sensors of $\mathrm{LOO}$ while the plot on the right covers the 36 remaining wide sensors. This is the reason behind the difference on Y-axis scales, displaying total values of $1000 \mu \mathrm{A}$ for the narrow sensors and $5000 \mu \mathrm{A}$ for the wides since, independently of its class, each sensor contributes with approximately $100 \mu \mathrm{A}$.

In the general picture the behavior of the current versus luminosity is roughly linear and for all the $\mathbf{L O O}$ modules there is a clear jump in the bias current data around $3000 \mathrm{pb}-1$. The increment on the bias current is also similar for each sensor and close to $20 \mu \mathrm{A}$. This anomaly has to be due to a global effect such as temperature rising in the detector. The CDF electronic logs show that at the luminosity corresponding to that time ( $3000 \mathrm{pb} \mathrm{b}^{-1}$, April 2007) the SVX was running warmer, from -10C to -6C. This temperature shift, even if small, could have affected the $\mathrm{LOO}$ since the reverse bias current is strongly dependent on temperature $\left(\mathbf{I}(\mathbf{T}) \propto \mathbf{T}^{2} e^{-E / 2 k T}\right)$. Besides, some time later ( $4500 p b^{-1}$, June 2008), the bias current data shows another jump also related to a cooling problem. The log revealed the possibility of an error in the termocouple or a temperature reading drifting in SVX that, 

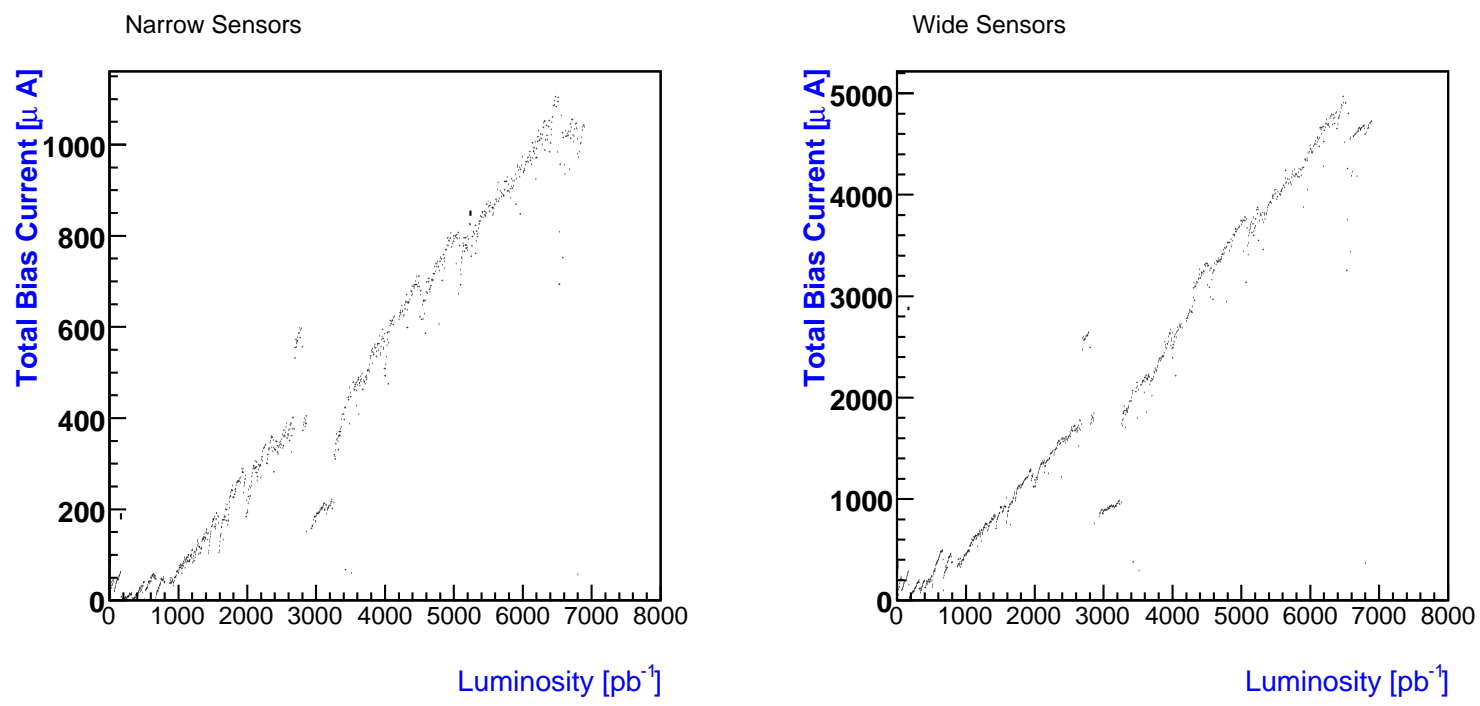

Figure 5.8: Total bias current measured on narrow sensors (left) and wide sensors (right).

again, affected L00. This incident may be useful as input to estimate the temperatures on SVX as read by LOO (indirectly through its bias current).

Another global effect on LOO is the presence of discontinuities in the bias current data plot. These discontinuities are long periods where the detector is turned off, for example annual shutdowns for maintenance work (scale of months, $100 \mathrm{pb}^{-1}$ ) or technical accesses (scale of days, $10 p b^{-1}$ ).

In a smaller scale we can observe different patterns and to notice them we have selected four interesting regions of Fig. 5.8 to zoom in:

- Region 1: from 0 to $1000 p b^{-1}$

- Region 2: from 1000 to $2500 p b^{-1}$

- Region 3: from 4000 to $5000 p b^{-1}$

- Region 4: from 5500 to $7000 p b^{-1}$

In Region 1 we can see short and periodically linear readings of increasing current. When the $\mathrm{LOO}$ is on and receiving radiation fluence the slope of the bias current is linear. When the detector is turned off the sensor material anneals and the bias current steps down to start rising again with similar slope after turning the sensors on. 

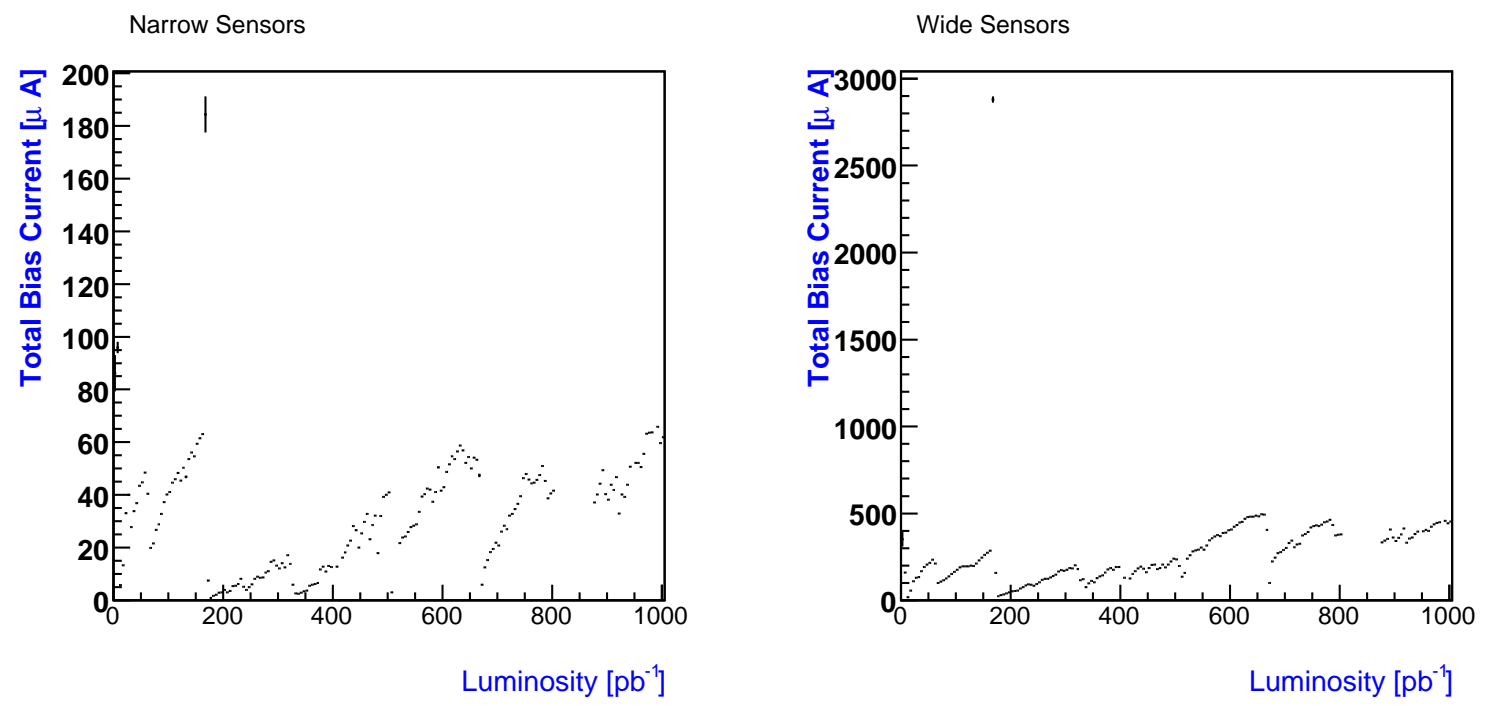

Figure 5.9: Region 1: zoom of Fig.5.8 in the range from 0 to $1000 \mathrm{pb}^{-1}$.

In Region 2 we can see upward periodical waves, not linear periods as in Region 1. This trend is typical before the discontinuity of $3000 \mathrm{pb}^{-1}$, that separates Region 2 from region 3 and may be related with high-starting luminosity and long Tevatron stores recordings.

Region 3 would be the equivalent figure to Region 2 after the discontinuity of $3000 \mathrm{pb}^{-1}$, although Region 3 does not seem to show such clear waves. The reason could be shorter quiet time between Tevatron stores and also high luminosity ones.

Region 4 shows the latest read data. After a long linear rising slope, the current steps down at $6500 \mathrm{pb}^{-1}$ (April 2009) and starts again what seems a new linear pattern, caused by a short-term stop in the data taking due to a technical access in the CDF vault. 

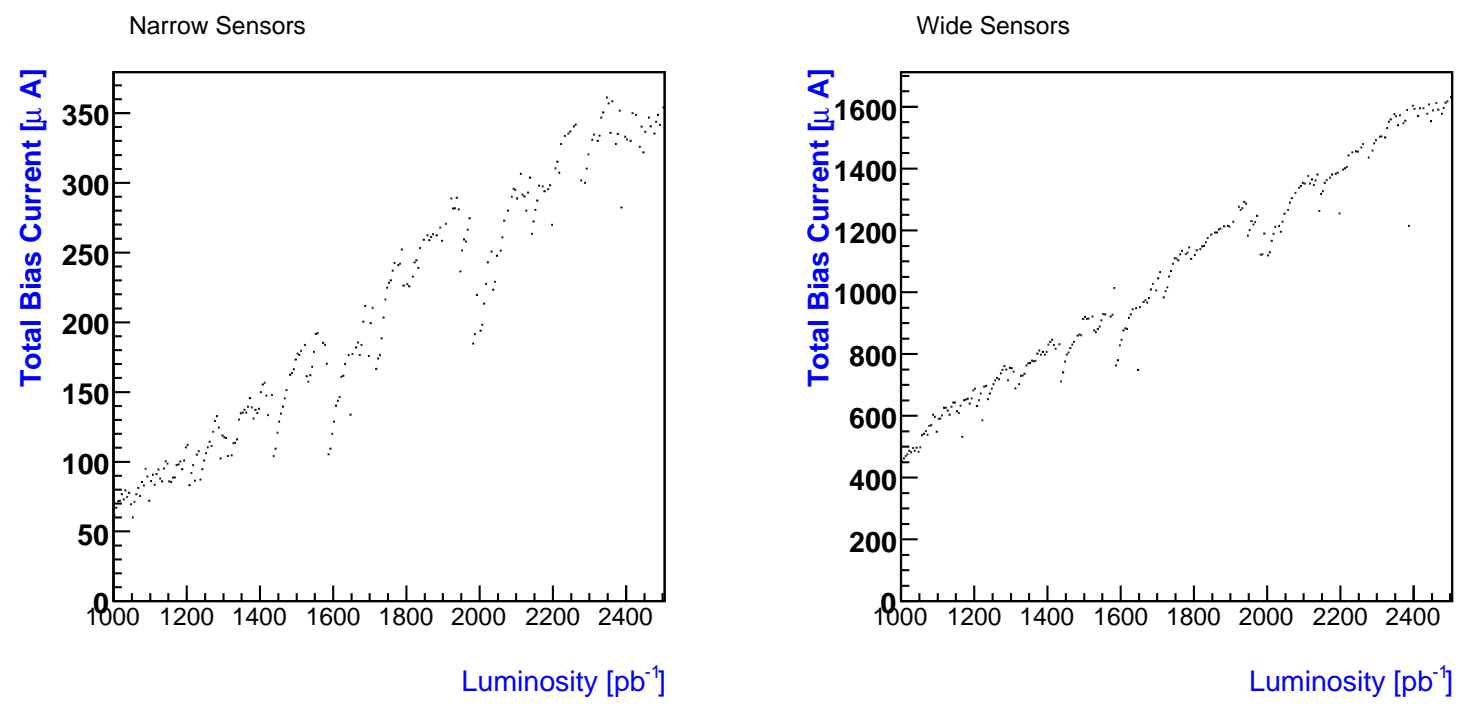

Figure 5.10: Region 2: zoom of Fig.5.8 in the range from 1000 to $2500 p b^{-1}$.
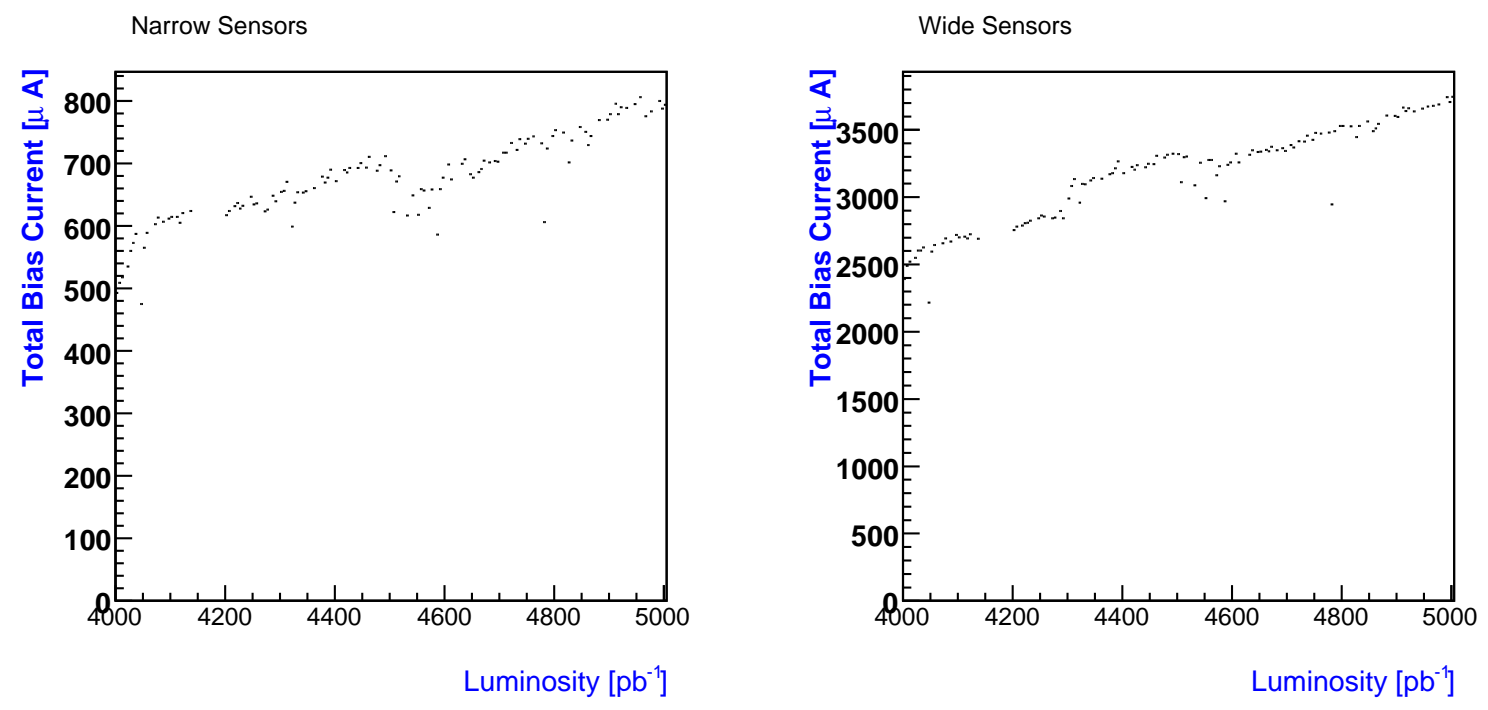

Figure 5.11: Region 3: zoom of Fig.5.8 in the range from 4000 to $5000 \mathrm{pb}^{-1}$. 

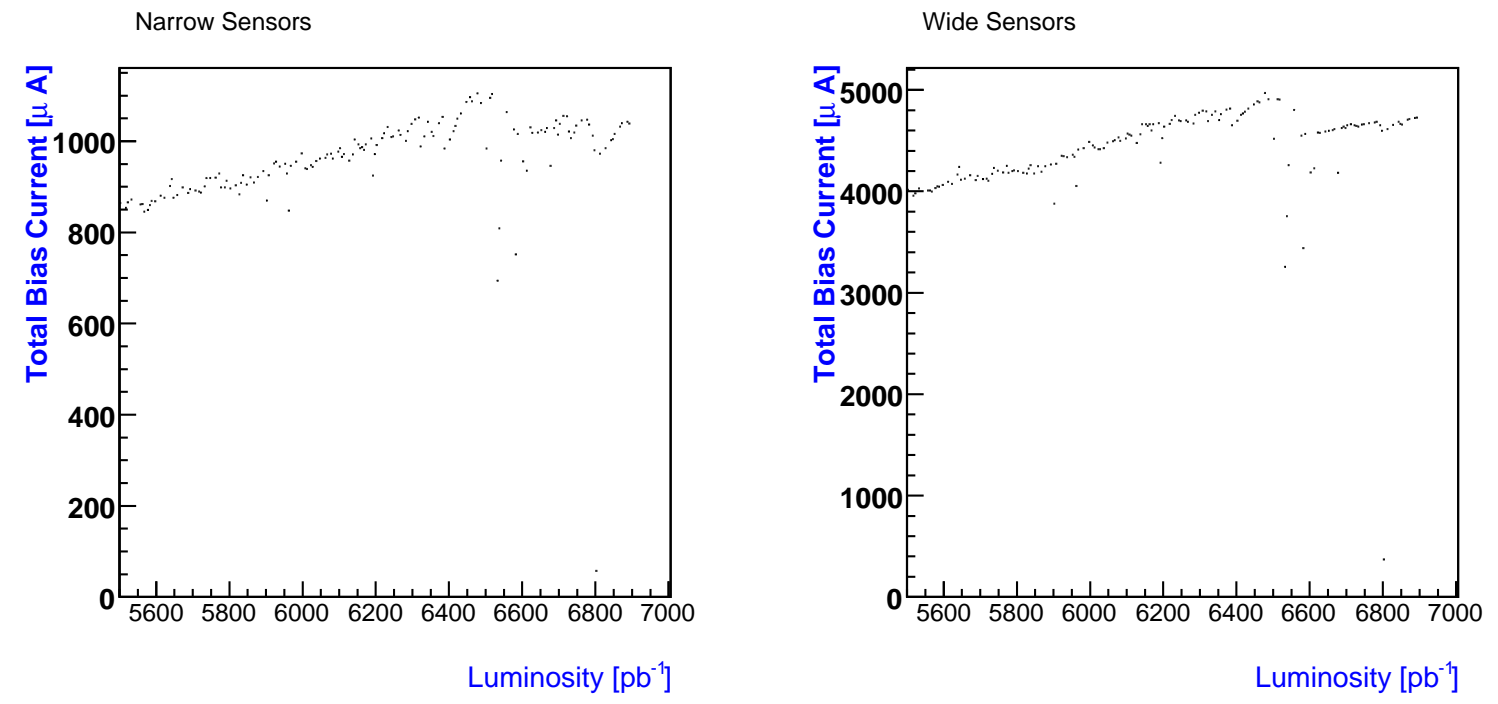

Figure 5.12: Region 4: zoom of Fig.5.8 in the range from 5500 to $7000 p b^{-1}$. 


\subsection{Luminosity to Fluence Conversion}

Data characterizing the degradation of the detector performance are typically displayed in luminosity unities in the $\mathrm{x}$-axis. Luminosity is a parameter of each particular collider. It is then a convenient expression if used in a local environment but not so useful in a global one. In the case of the Fermilab, for example, data expressed along luminosity would only be compared between the CDF and DO experiments, and the accelerator itself. If we were to compare data of type-inversion from the CDF with data from other experiment performed in a beam different from the Tevatron, we would need to convert the luminosity axe into fluence. Fluence is the radiative flux integrated over time. It is defined as the number of particles that intersect a unit area and hence a quantity independent of the origin of the source.

To proceed with this conversion ${ }^{1}$, data and parameters from Dong et al. [78] were shared. The equation used for the conversion is

$$
\Phi=L \cdot A \cdot r^{-\alpha}
$$

with constants $A=5 \cdot 10^{10}$ and $\alpha=1.65$. Variable $r$ is the radial distance from each sensor to the beam. To compute this distance it is needed to consider the position of the beam at each luminosity (Fig. 5.6).

\footnotetext{
${ }^{1}$ In this document the error in the measurement of the conversion to fluence has not been computed. One way to evaluate it would be by setting an $\alpha$ of 1.5 in the power law, as the TLD measurement suggests.
} 


\section{Chapter 6}

\section{Signal vs. Bias Scans and Detector Lifespan Extrapolations}

This chapter is dedicated to show the results obtained from LOO and SVX-LO. Those results (collected charge, delpetion voltage, evolution of depletion voltage, extrapolation for future behavior...) are based on data output by a periodical test performed to the detectors: the signal vs. bias scan.

\subsection{Bias voltage scans. The signal vs. bias scan implementation}

CDF regularly conducts scans to measure the depletion voltage of the sensors. Two different approaches are used for this purpose: the noise vs. bias scan and the signal vs. bias scan. The noise vs. bias scan takes advantage of the dependence of the $n$-side noise on the bias voltage. This method requires the use of double-sided sensors and hence it can be applied only to SVX-II or ISL. It will be discussed in section 6.3.

For the signal vs. bias scan the charge of hits on tracks is measured as a function of the bias voltage. For each bias voltage point, the peak of the charge distribution is derived fitting a Landau convoluted with a Gaussian resolution function. Then, all that set of points is fitted using a sigmoid function. The actual depletion voltage of the sensor is then defined as the $95 \%$ amplitude of the fitted sigmoid function (Fig. 6.1). This approach works for all sensors: L00, SVX-II and ISL. However, the disadvantage of this method is that it requires the consumption of valuable beam time.

Altough farther than L00 from the beam, CDF assumes that the Layer 0 of SVX-II is the most critical for the detector life span as LOO enjoys a more radiation-tolerant design due to its actively cooled sensors and its ability to work with higher bias voltages. 

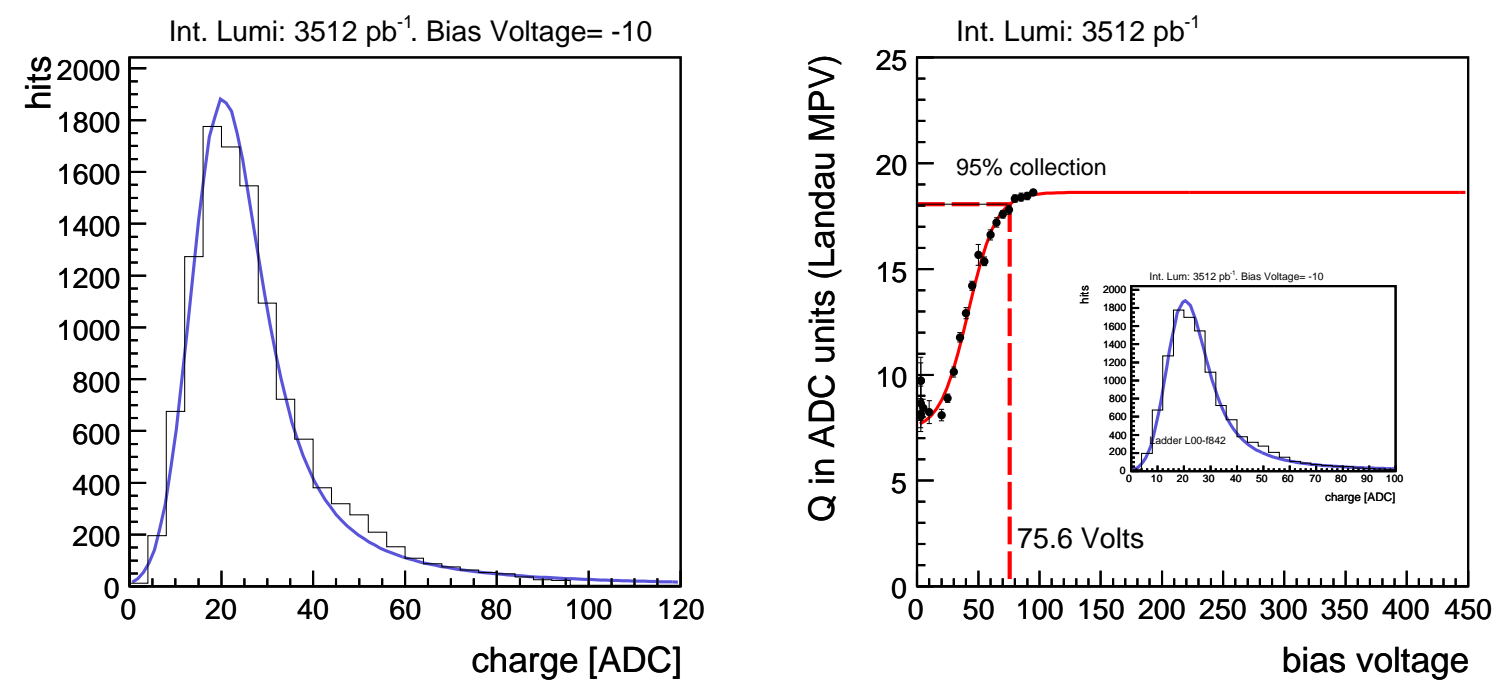

Figure 6.1: Example of the signal vs. bias scan method. This scan was performed on L00 at an integrated luminosity of $3512 \mathrm{pb}^{-1}$. The figure on the left shows the charge collection distribution (at the polarization voltage of 10 Volts below the nominal operating voltage) corresponding to one of the 48 modules of L00, and the Landau fit, characterized by its peak or Most Probable Value (MPV). The figure on the right displays the $25 \mathrm{MPVs}$ corresponding to the 25 different polarization voltages. This set of points is fitted to a sigmoid whose $95 \%$ of its height, 75.6 volts, is defined as the depletion voltage for this particular sensor and luminosity. The depletion voltage is the output point of the signal vs. bias scan method. 

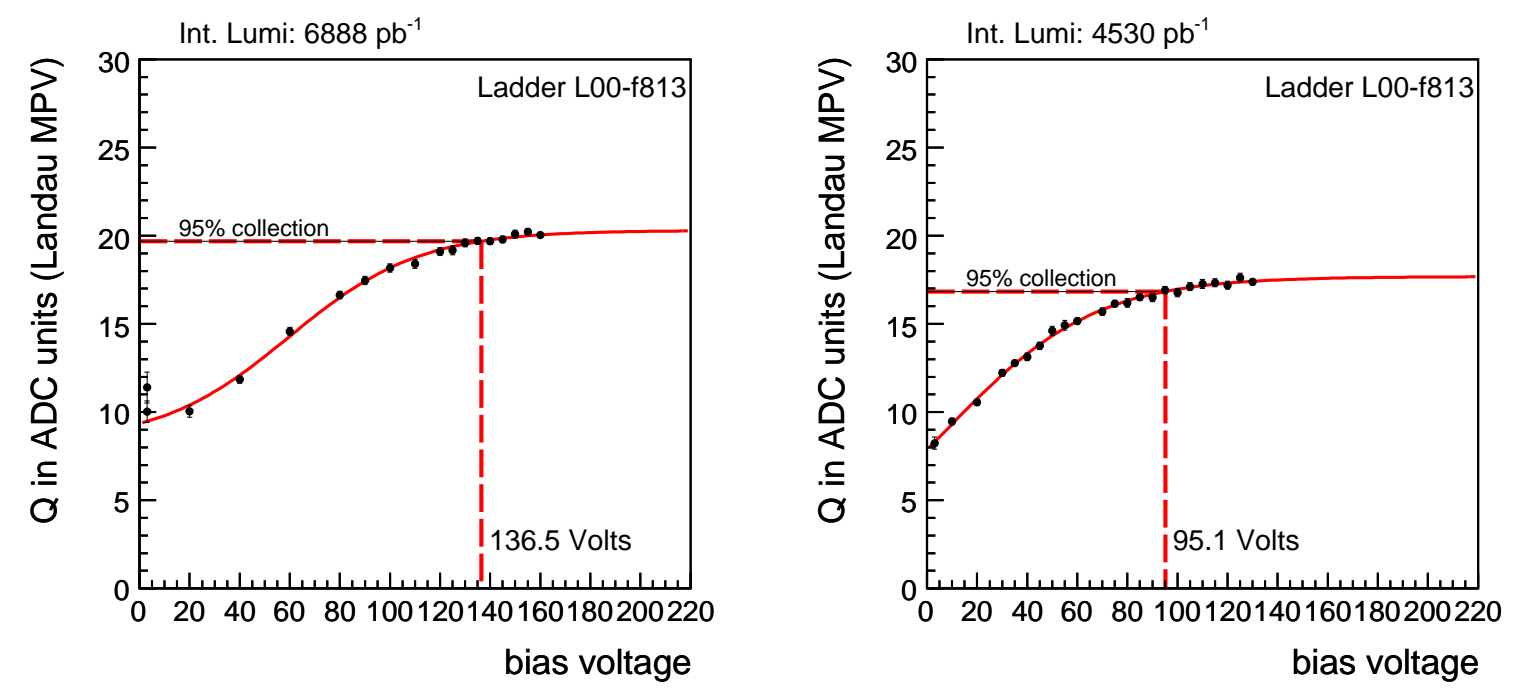

Figure 6.2: Depletion Bias plots for Micron class sensor f813.

\subsection{Results of the LO0 Signal vs. Bias scans}

\subsubsection{Study of the Depletion Voltage}

The Most Probable Values (MPV) of the Landau convoluted to Gaussian fits of the collected charge distributions that were recorded at different bias voltages, as discussed in the previous section, are displayed in figures 6.2, 6.3, 6.4, and 6.5 for 4 LO0 modules. The signal increases with bias voltage until reaching a plateau that we idetify with full bulk depletion. This set of points was fitted subsequently to a sigmoid curve. The $95 \%$ of the height of the saturation value of the sigmoid is defined as the depletion voltage of the particular sensor, and at the luminosity when the signal vs. bias scan test was performed.

Representative ladders for every sensor class were chosen. Their depletion voltages were compared at different luminosities of $7285 \mathrm{pb}^{-1}, 6888 \mathrm{pb}^{-1}$ and $4530 \mathrm{pb}^{-1}$. The differences between depletion voltages for those luminosities $\left(6888 \mathrm{pb}^{-1}\right.$ and $\left.4530 \mathrm{pb}^{-1}\right)$ was of 80.4 $\mathrm{V}$ for f843, $41.4 \mathrm{~V}$ for f813, and 36.9 and $41.3 \mathrm{~V}$ in the case of f821 and f841, respectively. Sensor f813 is a representative of the oxygenated radiation-hardened Micron sensors, and hence a small difference of depletion voltages between tests should be expected. A general view of the idea of different depletion voltages at different test luminosities is fully displayed with the help of the historical evolution plots. 

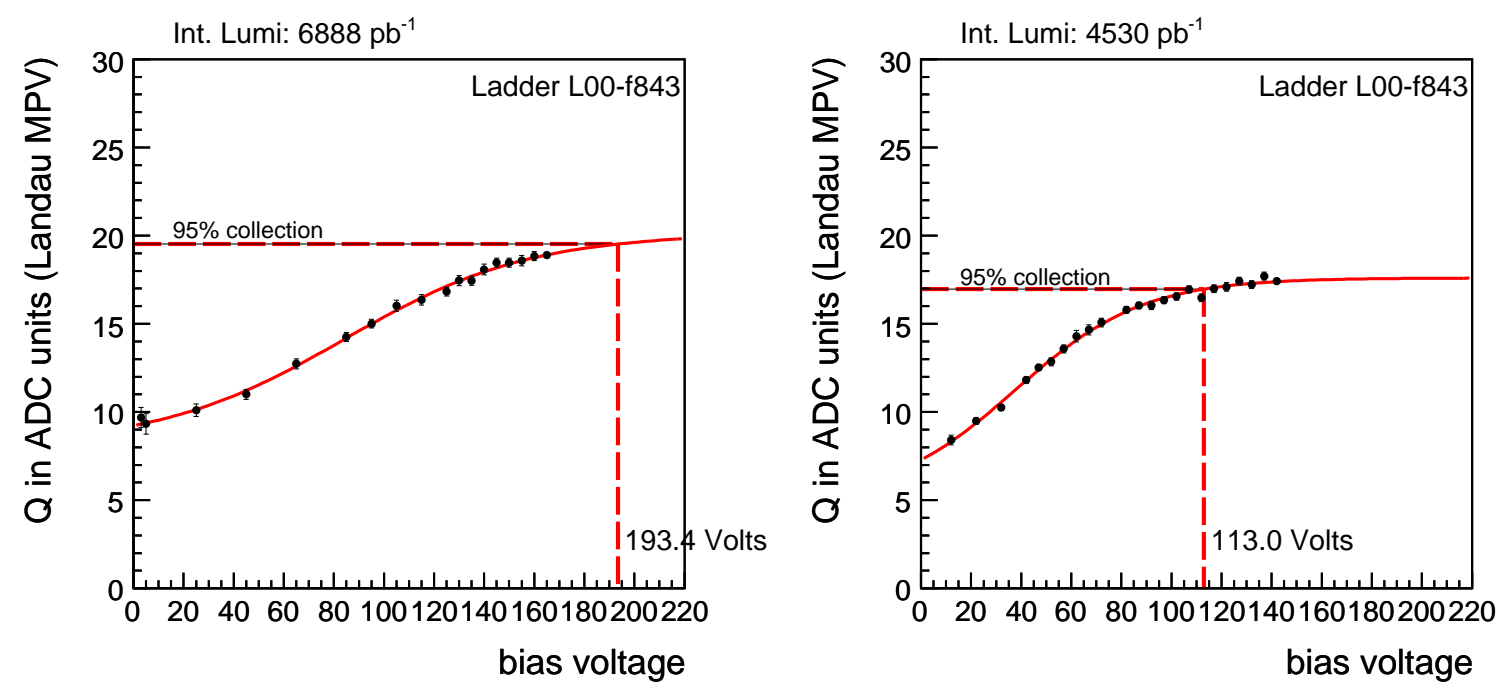

Figure 6.3: Depletion Bias plots for SGS Thomson sensor f843. 

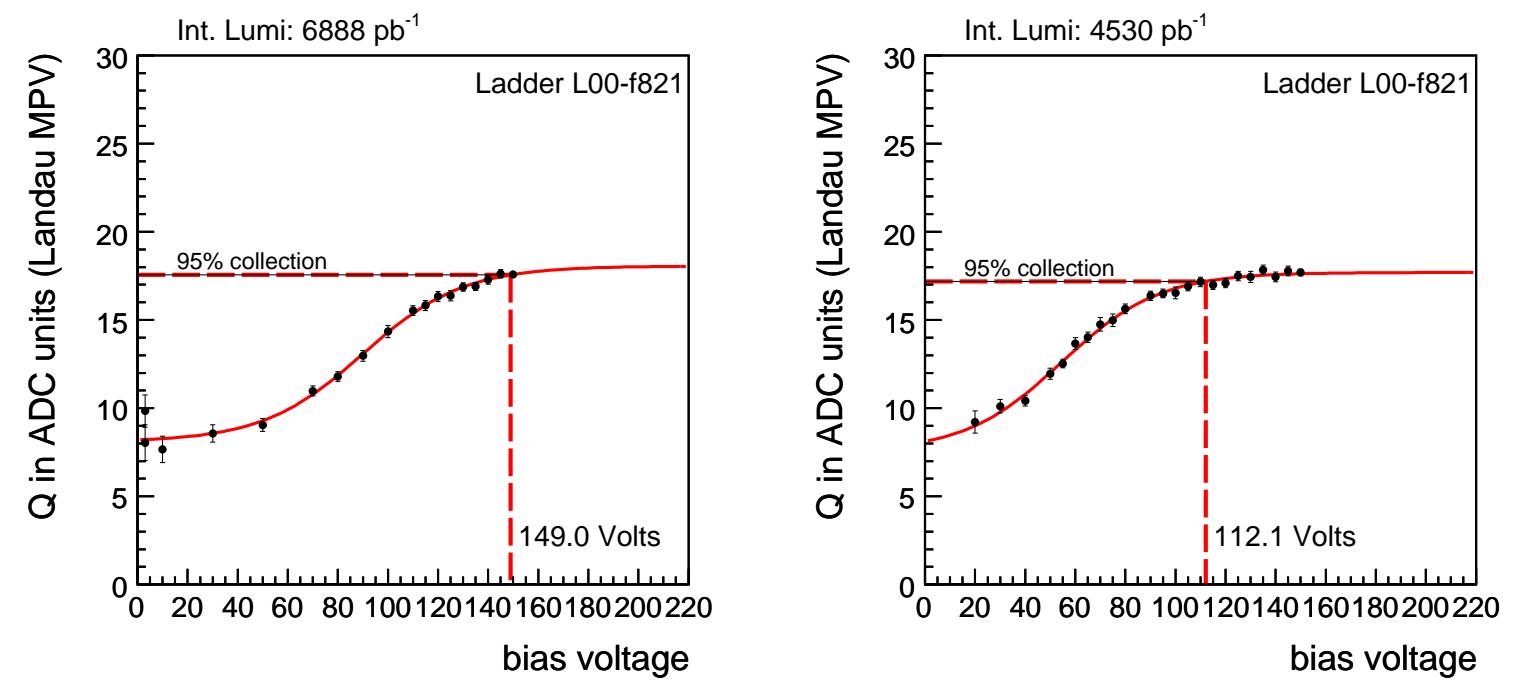

Figure 6.4: Depletion Bias plots for Hamamatsu sensor f821.
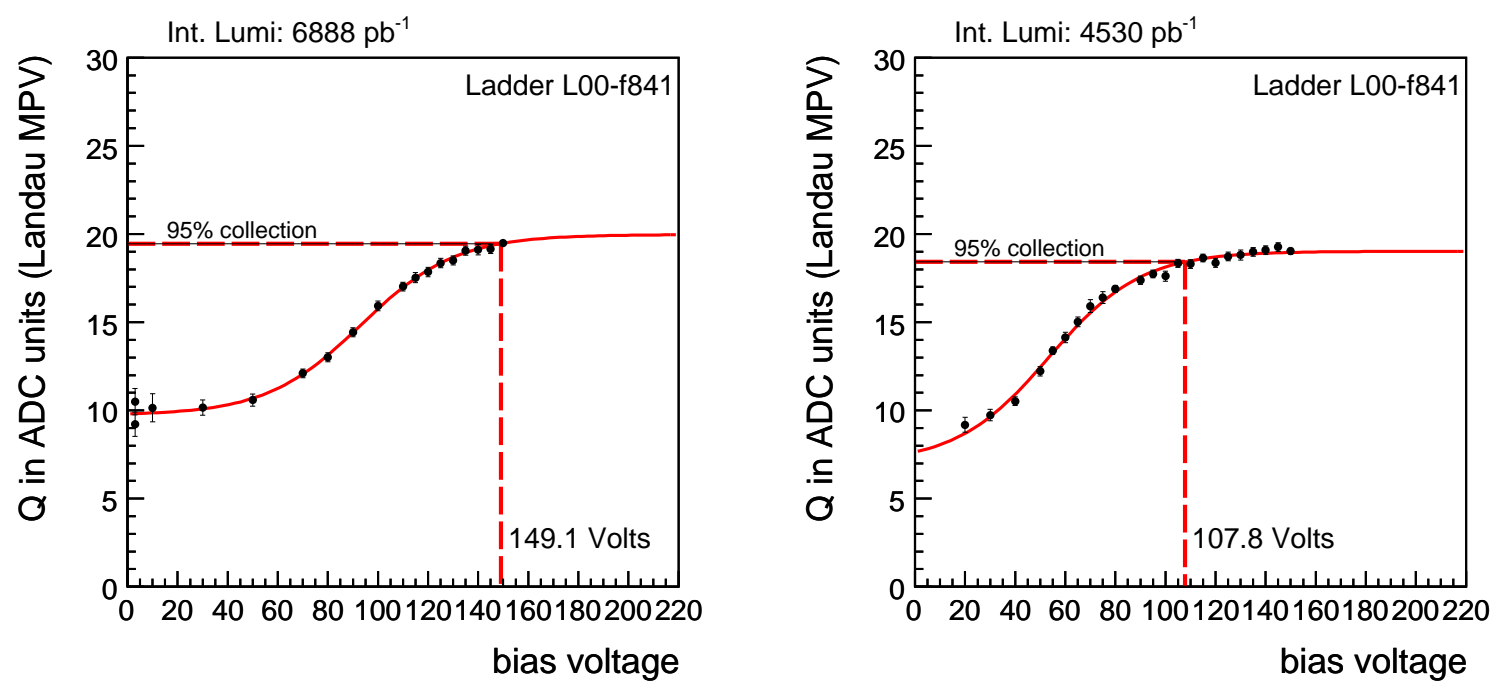

Figure 6.5: Depletion Bias plot for Hamamatsu sensor f841. 


\subsubsection{Evolution of the Depletion Voltage}

The monitoring of the behavior along time of a sensor is made by means of the observation of its depletion voltage evolution.

Modules for every one of the three representative sensor type have been selected: oxygenated Micron (Fig. 6.6), SGS Thomson (Fig. 6.8), and Hamamatsu (Figs. 6.10 and 6.12). In all these four figures the $x$-axis represents the integrated luminosity at which the signal vs. bias scans were taken. In the y-axis is represented the computed depletion voltage at the corresponding luminosity. Two fits have been performed: a third grade polinomial fit and a linear fit. The polinomic curve fits the set of points of low luminosity. For every sensor, we have defined the inversion point as the minimum of the polinomic curve. The $(x, y)$ coordinates of the inversion point are respectively the inversion luminosity and the inversion depletion voltage. The linear fit has been performed for the subset of points beyond the inversion point as an extrapolation of the behavior of the ladder. Figures 6.7, 6.9, 6.11, and 6.13 are the equivalent plots expressed as a function of the fluence. Figures 6.16 and 6.17 are distributions of the inversion point in terms of luminosity and fluence, respectively. Figure 6.16 shows the $x$ and $y$ coordinates of the inversion point while 6.17 shows the $x$ coordinate and the linear fit slope. It is interesting to compare the distributions of x-coordinate for luminosity and its equivalent for fluence (histograms MinXLum and MinXFlu), since the fluence transformation can perfectly resolves the three peaks corresponding to the three classes of sensors of $\mathrm{LOO}$.

Figure 6.18 shows that oxygenated Micron sensors delayed the type-inversion phenomenon, whose inversion luminosities are located around $2500 \mathrm{pb}^{-1}$ (corresponding to a fluence of $\left.75 \times 10^{12} \mathrm{~cm}^{-2}\right)$. Standard SGS Thomson and Hamamatsu sensors display inversion luminosities around $1500 \mathrm{pb}^{-1}\left(50 \times 10^{12} \mathrm{~cm}^{-2}\right)$ and $1000 \mathrm{pb}^{-1}\left(25 \times 10^{12} \mathrm{~cm}^{-2}\right)$, respectively. The subset of points beyond the inversion point seems to display a linear behavior for the three classes of sensors of $\mathrm{LOO}$. 

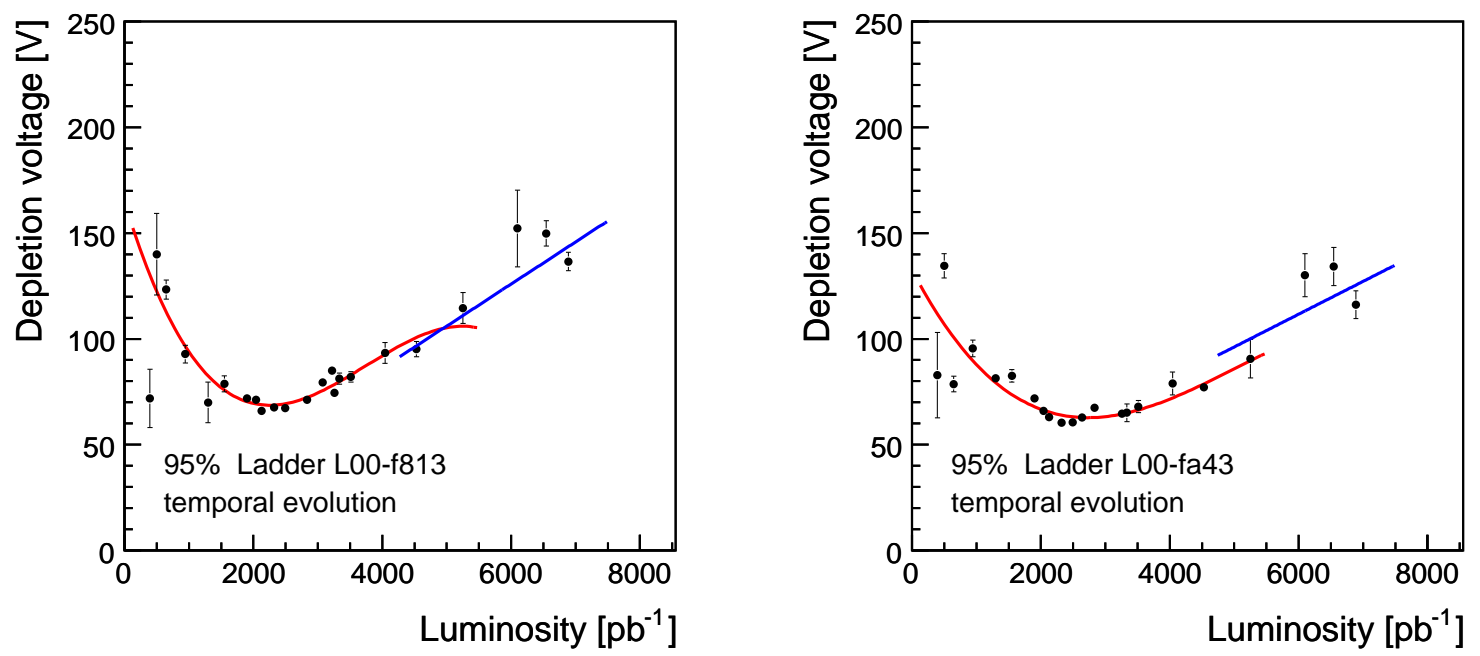

Figure 6.6: Example of inversion of the Micron class sensors f813 and fa43 (luminosity data).
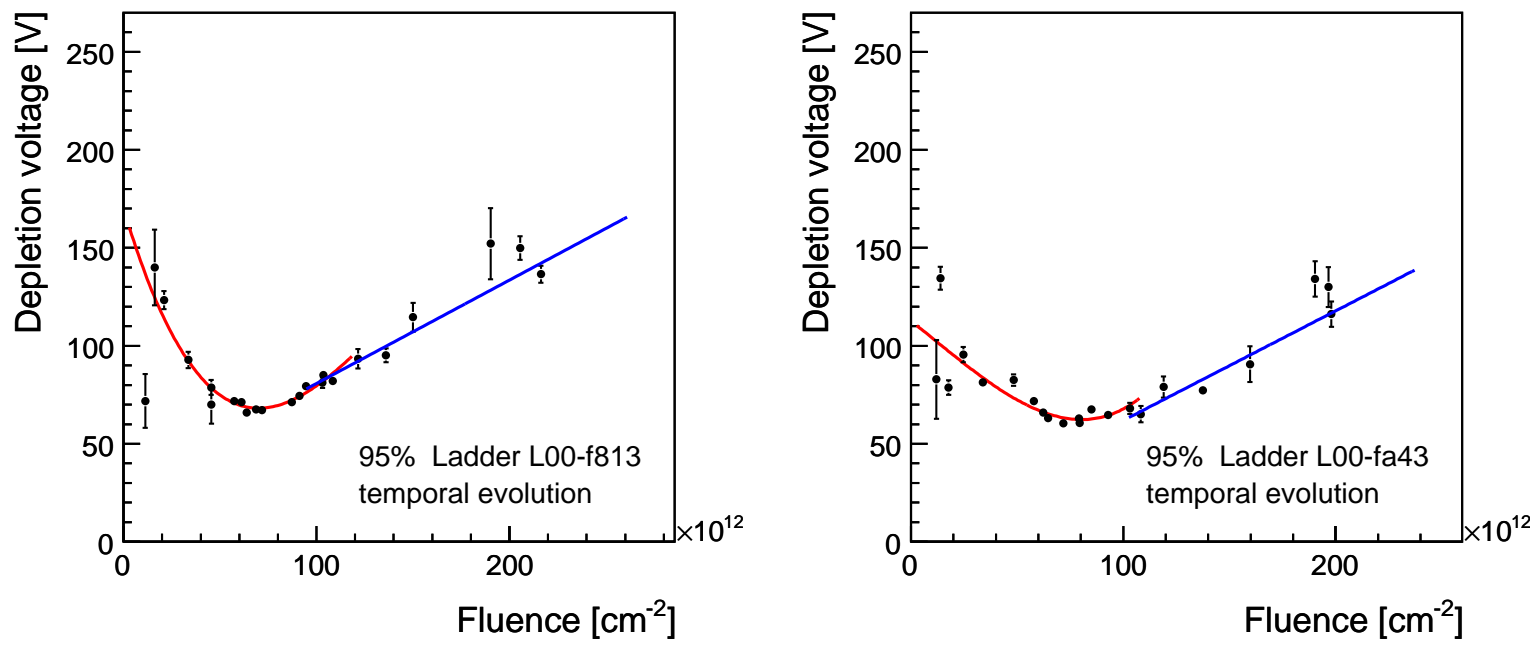

Figure 6.7: Example of inversion of the Micron class sensors f813 and fa43 of L00 (fluence data). 

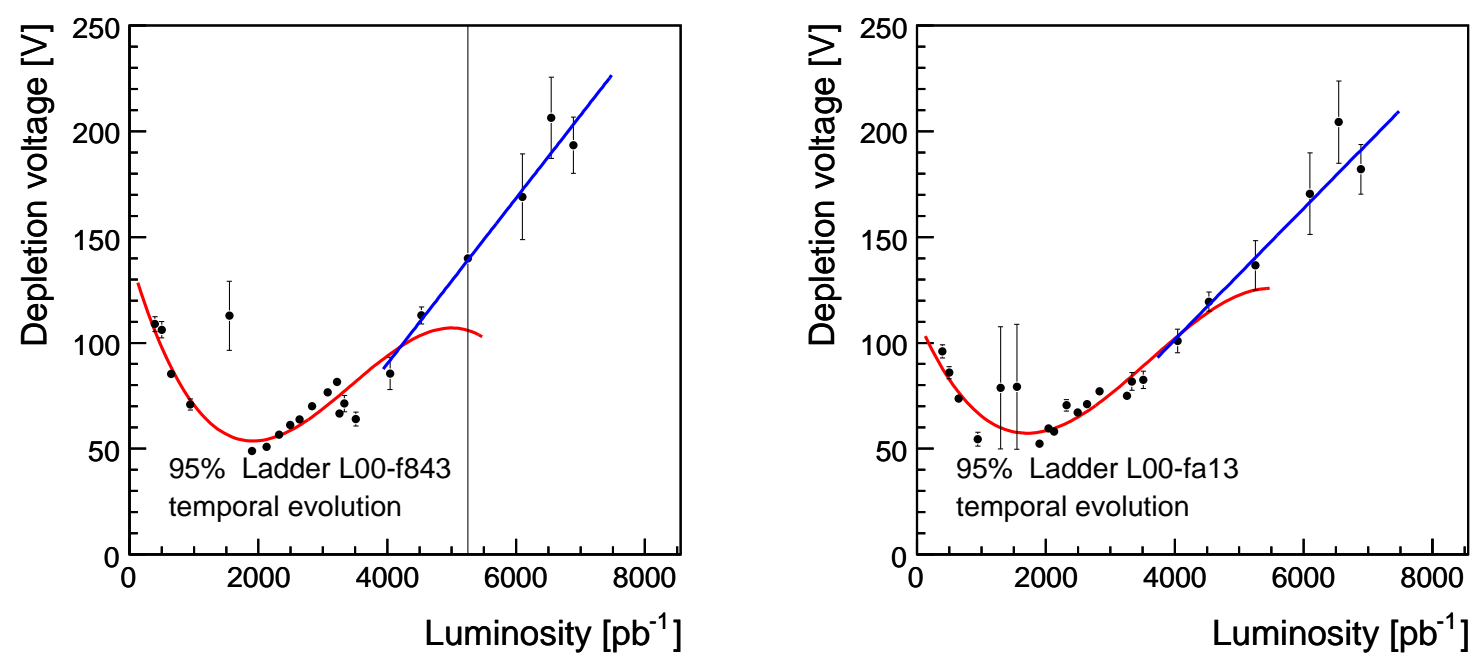

Figure 6.8: Example of inversion for SGS Thomson sensors f843 and fa13 (luminosity data).
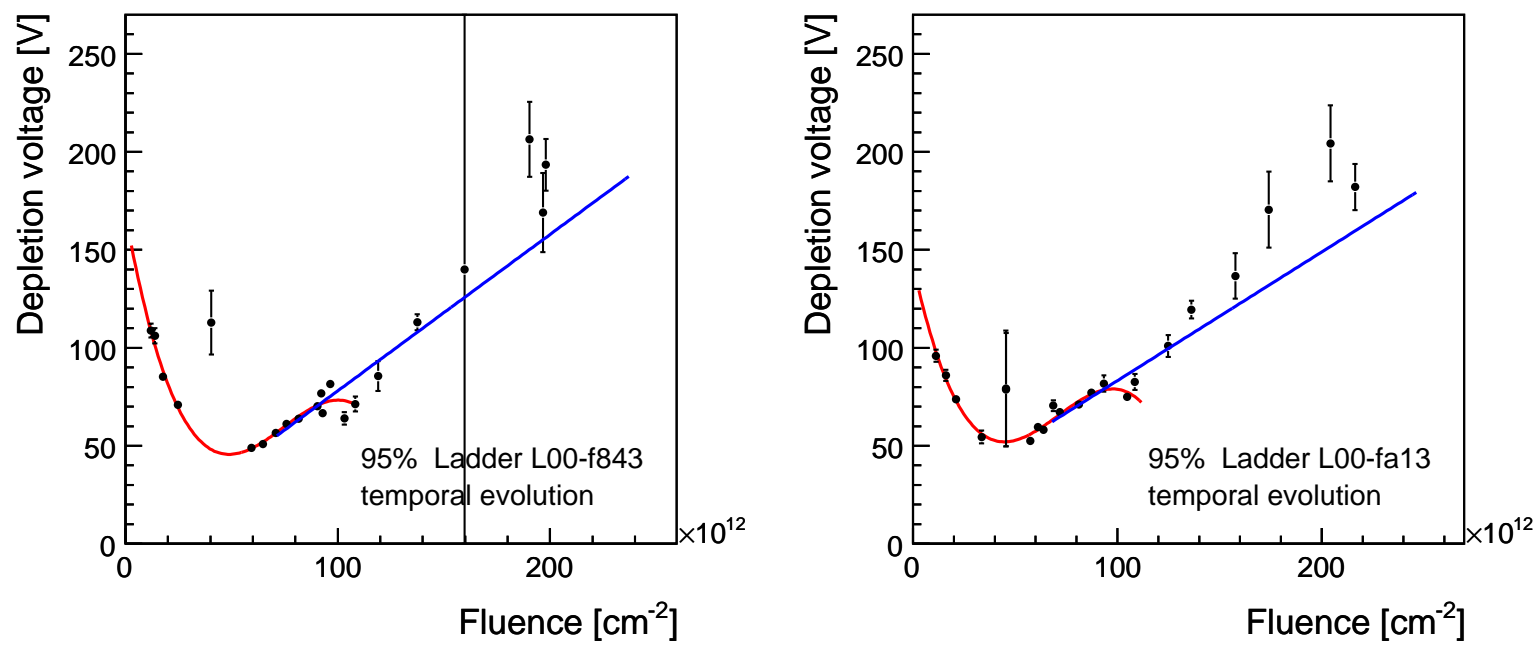

Figure 6.9: Example of inversion for SGS Thomson sensors f843 and fa13 (fluence data). 

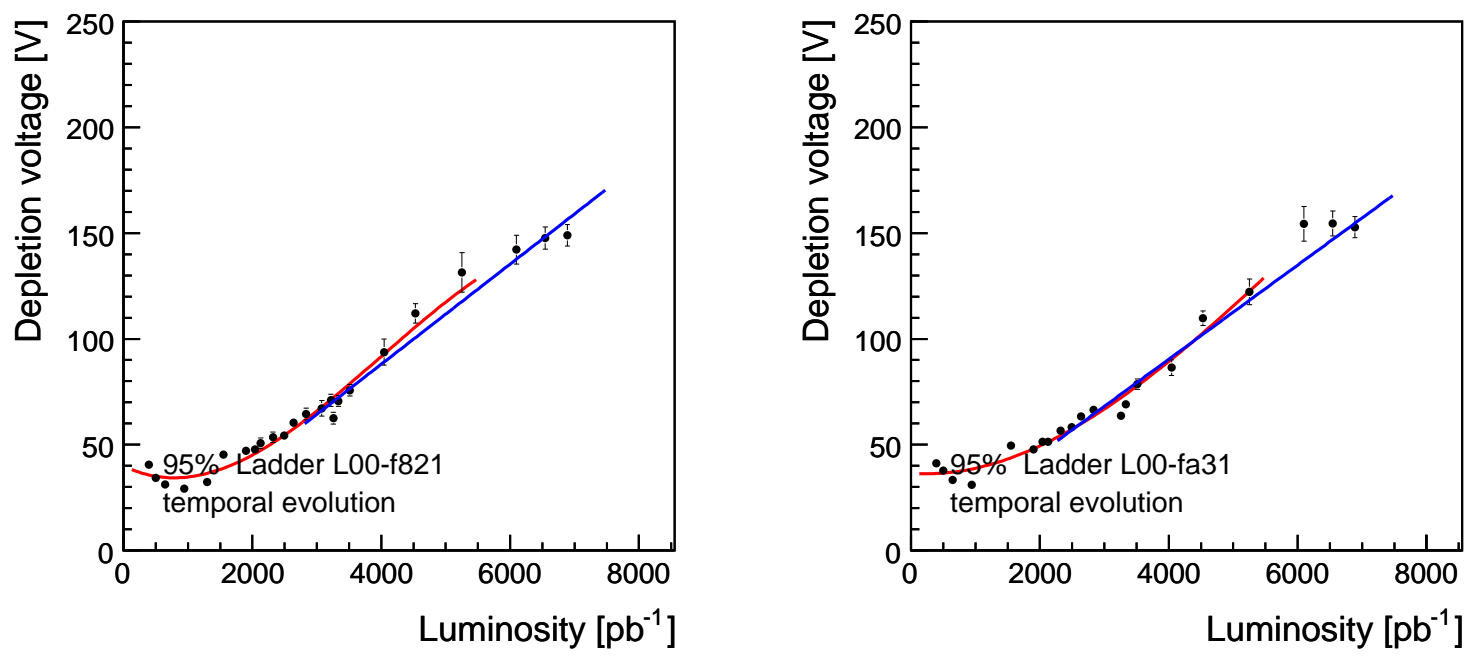

Figure 6.10: Example of inversion for Hamamatsu sensors f821 and fa31 (luminosity data).
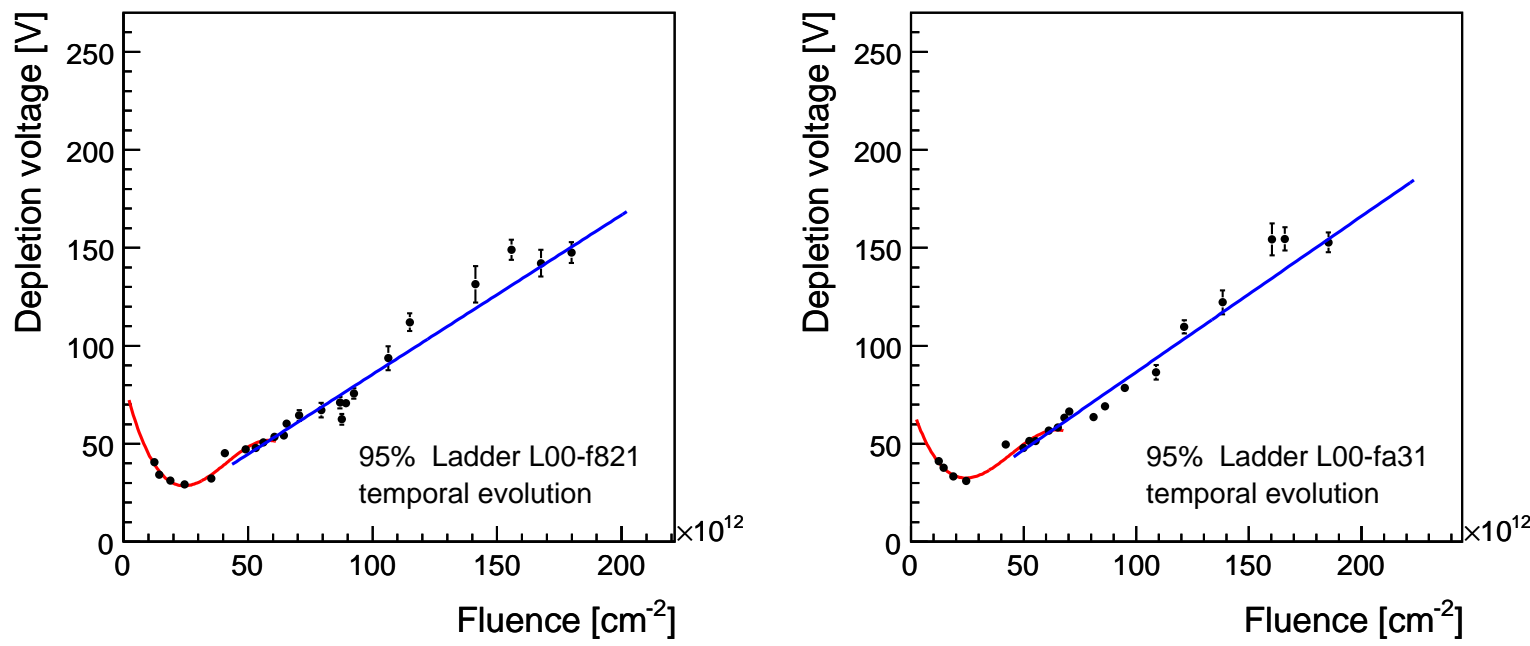

Figure 6.11: Example of inversion for Hamamatsu sensors f821 and fa31 (fluence data). 

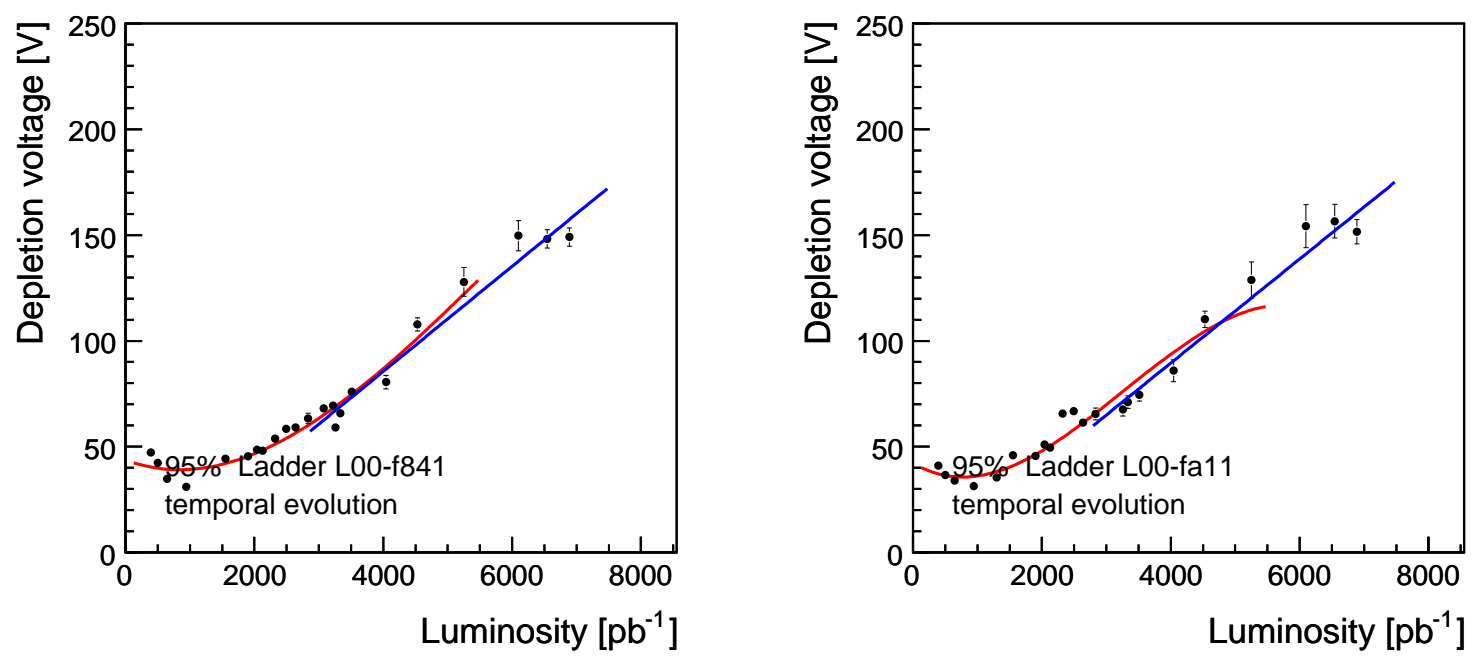

Figure 6.12: Example of inversion for Hamamatsu sensors f841 and fa11 (luminosity data).
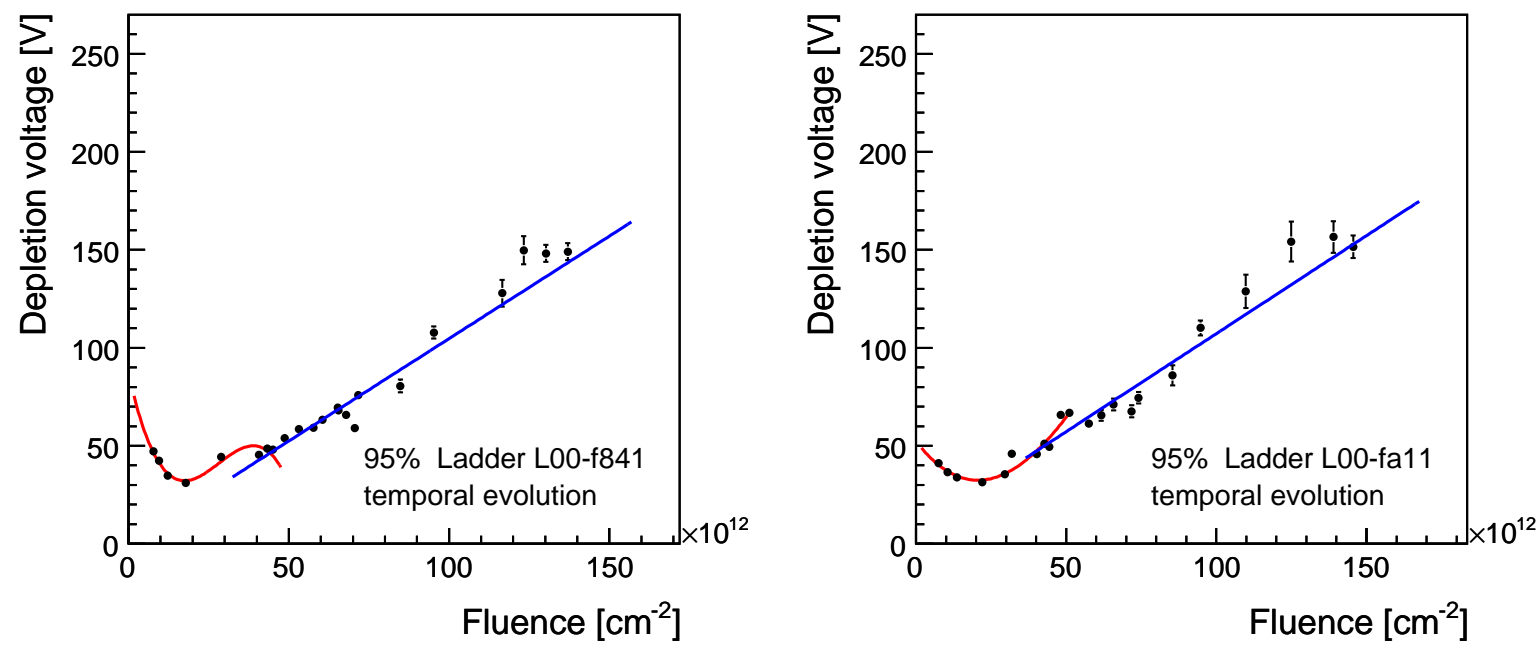

Figure 6.13: Example of inversion for Hamamatsu sensors f841 and fa11 (fluence data). 

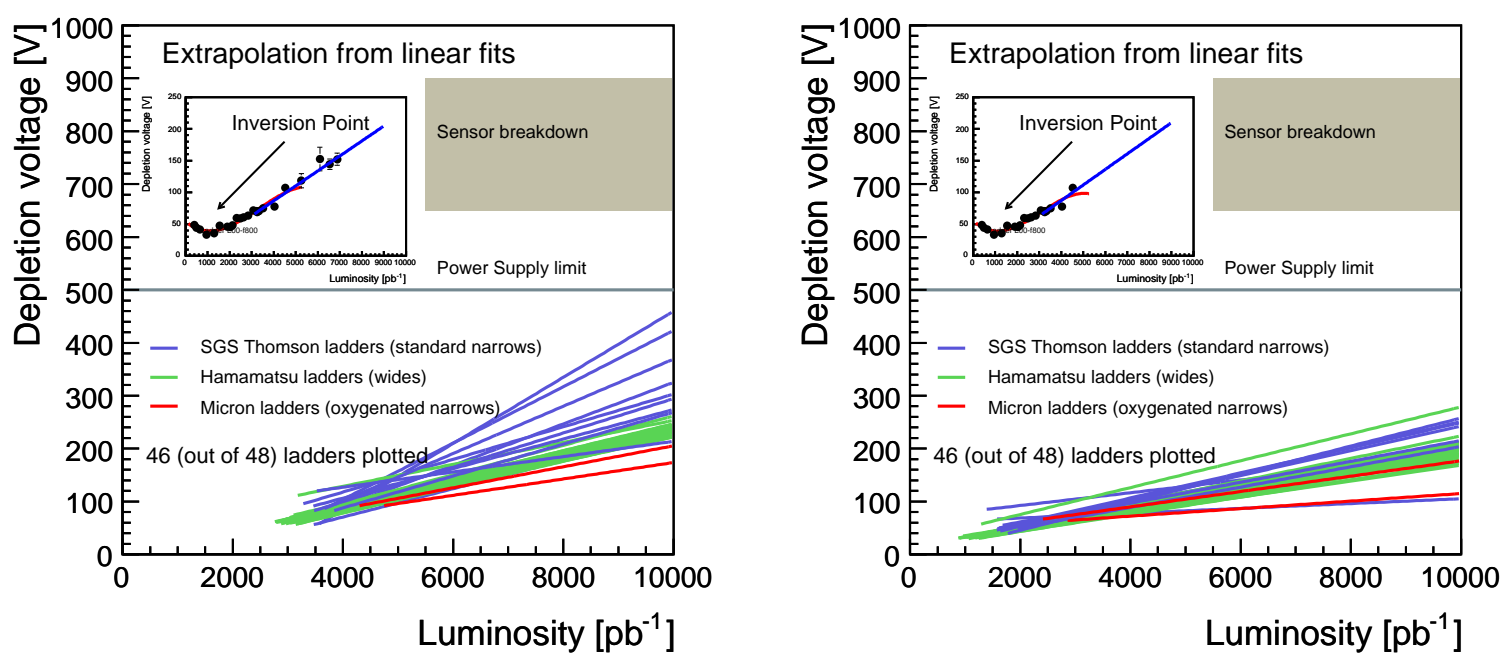

Figure 6.14: Global results for L00 luminosity plots. Summary of extrapolation fits of L00 at latest luminosity of $6888 \mathrm{pb}^{-1}$ (left), and at previous luminosity of $4530 \mathrm{pb}^{-1}$ (right).

\subsubsection{L00 Extrapolations Summary}

The extrapolation summary plot for LO0 (Fig. 6.14) displays together all the extrapolated linear fits corresponding to the 48 readout devices of L00. The plot also shows the technical limits of LO0 sensors: the power supply delivery limit and the sensor breakdown region. Figure 6.14 shows the extrapolated fits after data collection up to two different luminosities, $6888 \mathrm{pb}^{-1}$ and $4530 \mathrm{pb}^{-1}$, to compare the evolution of the extrapolation's slopes. If the slopes at two different luminosities show significative differences, the assumption of a linear extrapolation after type-inversion should be revised.

We observe that all of the extrapolated fits lie below the power supply limit of $500 \mathrm{~V}$ and the breakdown region. With the actual results we conclude that the sensors will be able to be depleted at least to a luminosity of $10000 \mathrm{pb}^{-1}$.

Figure 6.15 is a global display of L00 in terms of fluence. The geometrical arrangement is slightly altered in both plots of Fig. 6.15 to include the Micron and SGS Thomson sensors $(f 8 * 3)$. Coordinates of this sensors are located in the columns correspondidng to $z=1$ and $z=8$. The inversion point for this kind of sensors is located around a fluence of $40 \cdot 10^{12}$ $\mathbf{c m}^{-2}$ and for the Hamamatsu devices this value lies about $20 \cdot 10^{12} \mathbf{c m}^{-2}$. The slopes of the linear fits of the LOO Micron sensors (Fig. 6.15, right) seem to be higher in the western region altough to affirm this, more statistics or a diffrerent set of data would be needed. 
LO0

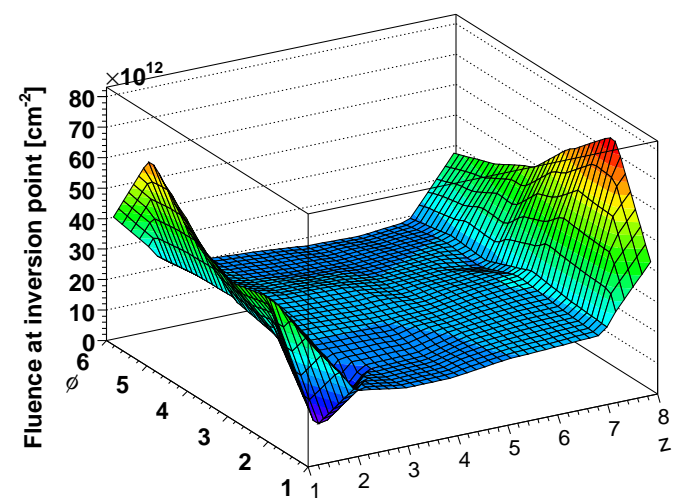

LOO

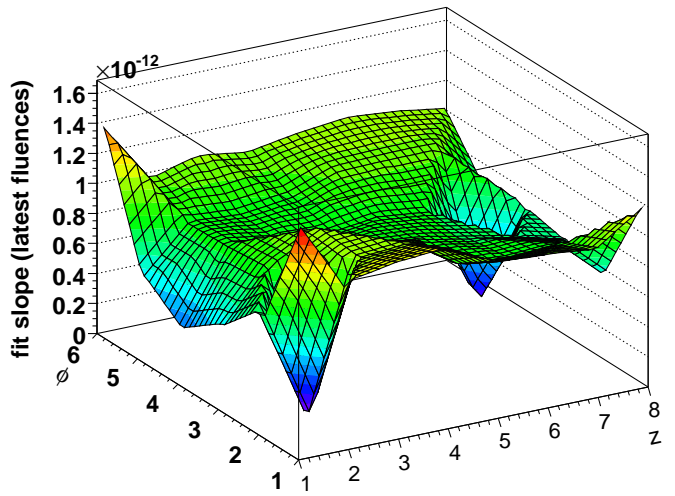

Figure 6.15: Global results for L00 fluence plots. Summary for L00 sensors of abscises of the inversion point (left) and linear fits slopes (right). Geometrical interpretation is altered to include the Micron sensors ( $f 8 * 3$ ). Coordinates of this sensors lie in the plane corresponding to columns of $z=1$ and $z=8$.

\section{MinXLum}

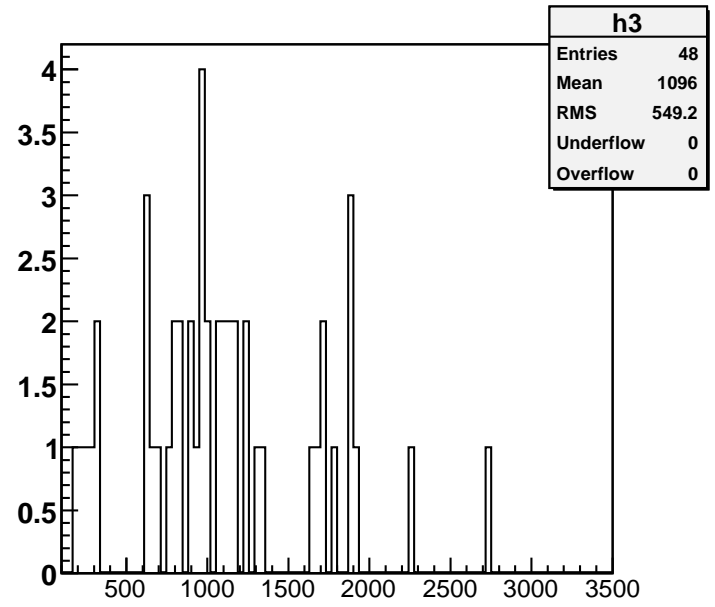

\section{MinYLum}

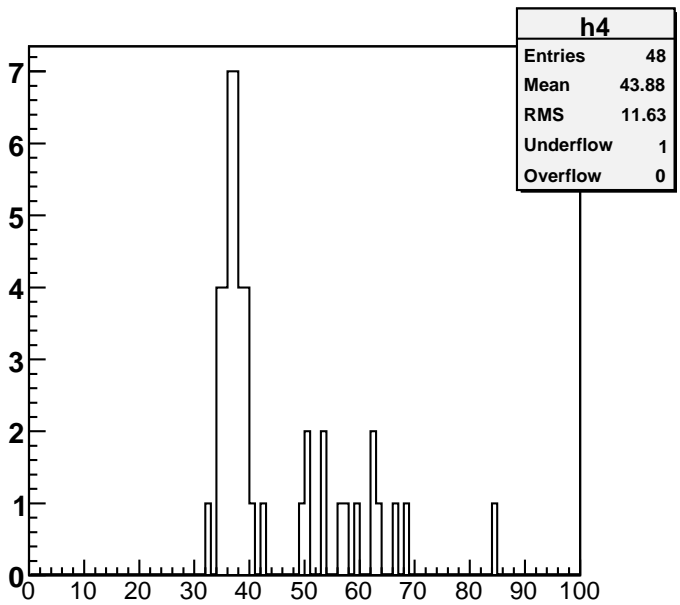

Figure 6.16: Distribution of the coordinates of the inversion point for L00 sensors (luminosity version). Left, $x$-coordinate; right, y-coordinate. The existence of two peaks of populations is expected since different classes of L00sensors invert at different points. 
MinXFlu

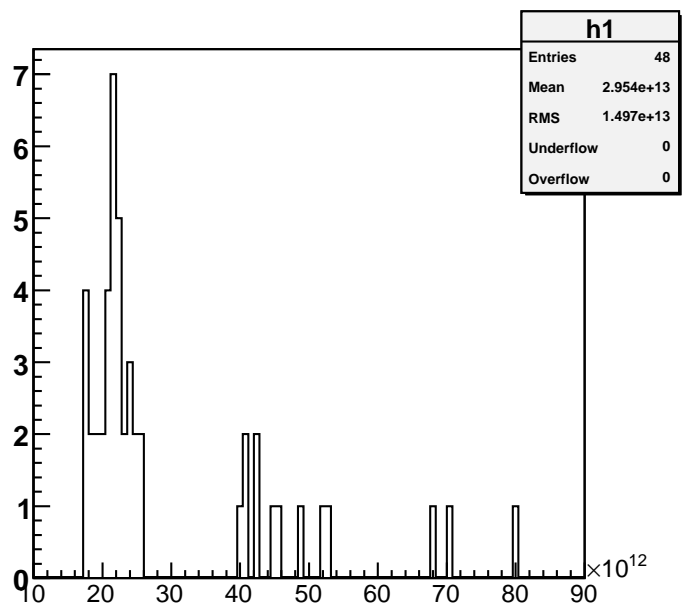

SloFlu

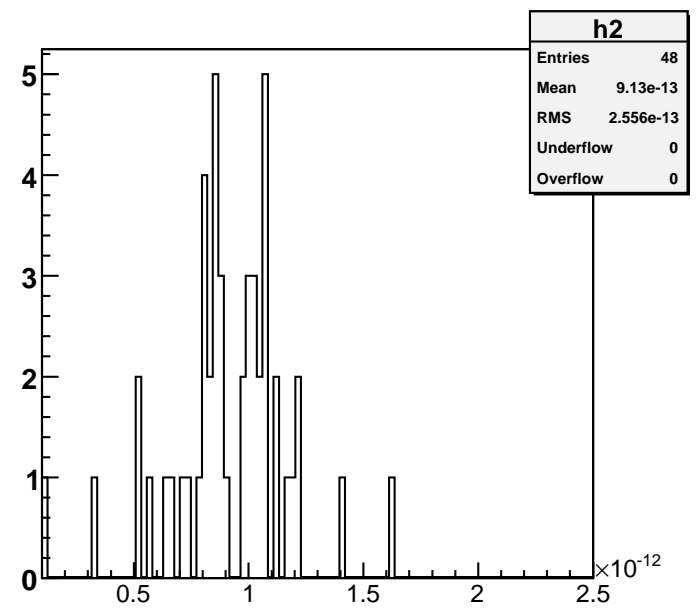

Figure 6.17: Distribution of the x-coordinate of the inversion point (left) and linear fit slope for points after inversion (right) (fluence version) for L00 sensors (fluence version).

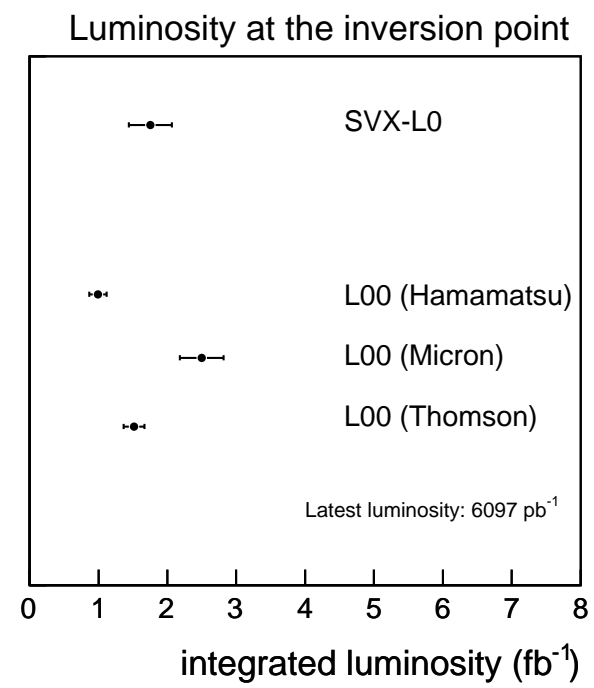

Figure 6.18: Global results for L00 and SVX-L0 luminosity plots. Comparison of inversion luminosities for the different classes of sensors of L00 and SVX-L0. Results were computed after data taken at a latest luminosity of $6097 \mathrm{pb}^{-1}$. 


\subsection{Results of the SVX-LO Signal vs. Bias scan}

\subsubsection{Study of the Depletion Voltage}

Readout devices e160 and e460 were chosen as representatives of SVX-LO. The depletion voltage plots of these devices (Fig. 6.19 and 6.20) are shown after being computed from latest data at $6888 \mathrm{pb}^{-1}$. The plots are made up of a single set of points and its fit. The set of points are the Most Probable Values (MPV) of the Landau convoluted to Gaussian fits of the charge collection distributions that were recorded at different bias voltages. The set of points was fitted to a sigmoid curve. similarly to the previous case of $\mathrm{LO0}$, the $95 \%$ of the height of the saturation value of this sigmoid is defined as the depletion voltage of the particular sensor and at the luminosity when the signal vs. bias scan test was performed.

In the depletion voltage plots it can be observed that the charge collection for $z$ sides is lower in approximately 10 ADCs than for the $\phi$ sides. This difference is fully displayed in the plots of historical evolution of charge (Fig. 6.25). This is a result ofthe induced capacitance due to the double metal layer required for the $90^{\circ}$ double-sided sensors.
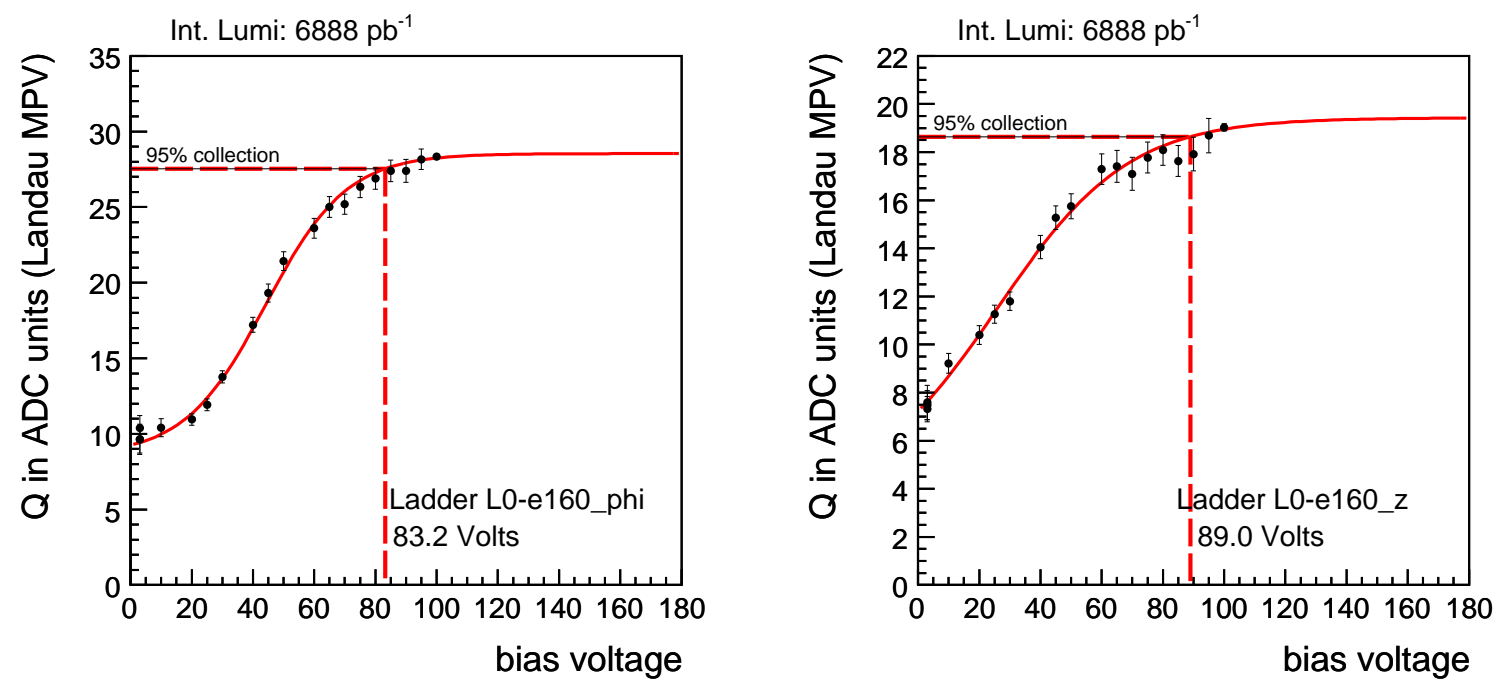

Figure 6.19: Depletion voltage for $\phi$ (left) and $z$ (right) sides of e160 ladder at $6888 \mathrm{pb}^{-1}$. 

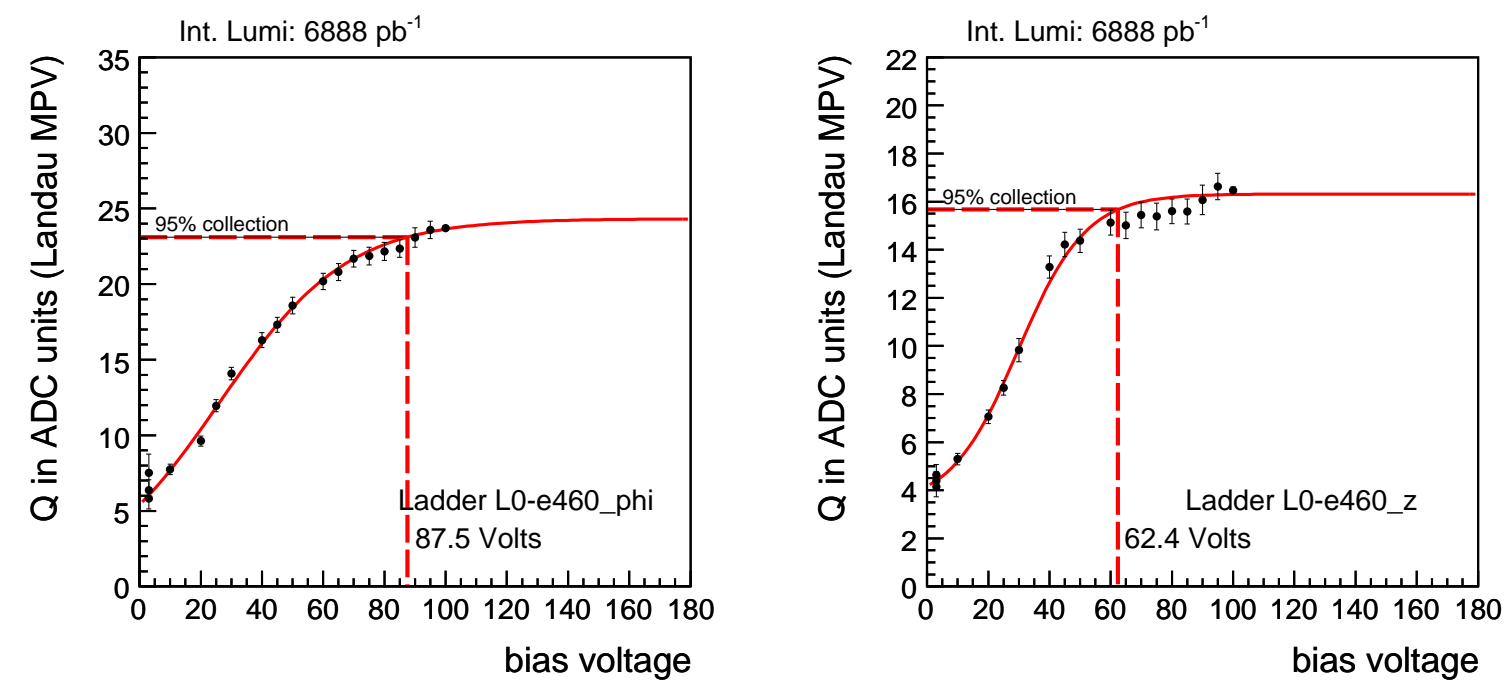

Figure 6.20: Depletion voltage for $\phi$ (left) and $z$ (right) sides of e460 ladder at $6888 \mathrm{pb}^{-1}$.

\subsubsection{Evolution of the Depletion Voltage}

Sensor e160 has been selected to illustrate the time evolution of the depletion voltage of SVX-L0. Its historical evolution of depletion voltage (Figs. 6.23, 6.24) and collected charge in full-depletion mode (Fig. 6.25) are shown in Figures 6.23 and 6.25, respectively. In Figure 6.25 it is possible to observe that for both sides of the sensor, collected charge has decreased about $10 \%$ of its original value at luminosity of $2000 \mathrm{pb}^{-1}$.

Signal vs. bias scans before luminosity of $3000 \mathrm{pb}^{-1}$ were performed only to three (e140, e2a0, e090) of the 72 sensors of SVX-L0. In spite of this lack of statistics, the evolution of the depletion voltages of those three sensors is in qualitative agreement with the prediction model (Fig. 6.26) as described in Ref. [66].

Observing that the subset of points of more recent luminosities (i.e. after $3500 \mathrm{pb}^{-1}$ ) display increasing values, we can assume that all the SVX-LO sensors have undergone type inversion. A linear fit was performed on that subset of points (Fig. 6.23) and its extrapolation is used to extract conclusions about the SVX-LO future behavior.

The noise bias scan makes use of the $n+$ noise drop achieved when the detector is fully depleted (Fig. 6.21). The bias voltage is set to different values and the depletion voltage is defined as the point where the noise reaches a minimum. The $n+$ strips of an undepleted double-sided microstrip detector have a large noise level since, in the absence of polariza- 


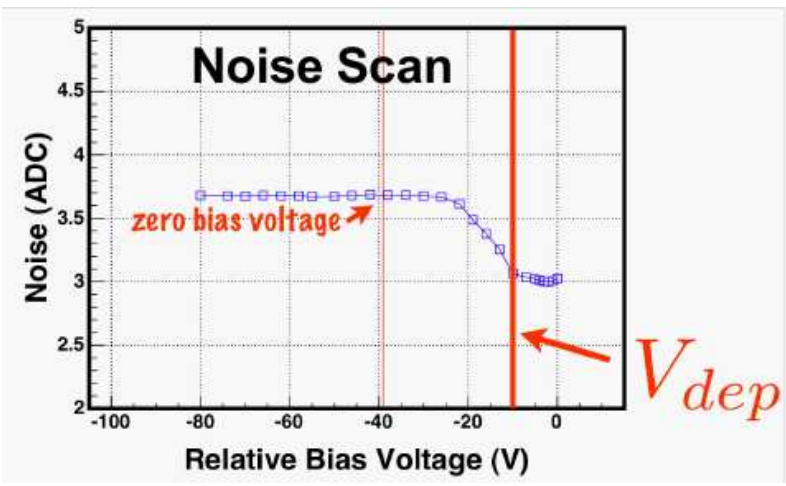

Figure 6.21: Method of noise vs. bias scan. This method is valid only for double-sided sensors.

tion, all backplane strips are connected. The strips are isolated through $p$ doped strips deposited within the $n+$ strips, the so-called $p$-stops. When the charge depleted region reaches the backplane, the $p$-stop are reverse-biased introducing a large resistance between neighbouring $n+$ strips and thus the noise drops. However, after inversion the assumption that the sensor starts to deplete from the $p$-strips is no longer valid making thus this method unreliable, as can be seen from Fig. 6.22. 

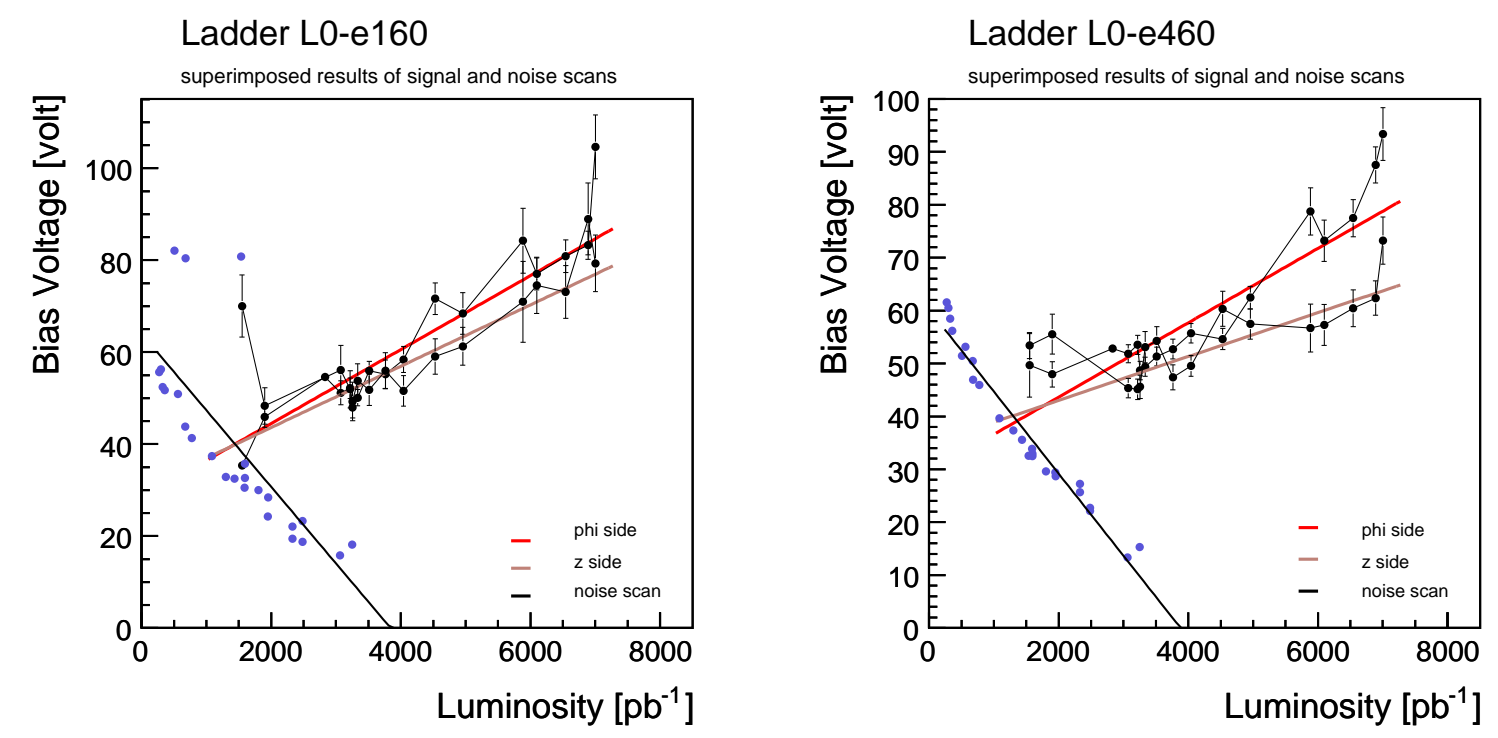

Figure 6.22: Signal vs. bias and noise vs. bias combined results for sensors e160 and e460. The noise scan method is only reliable for the region before type-inversion. 

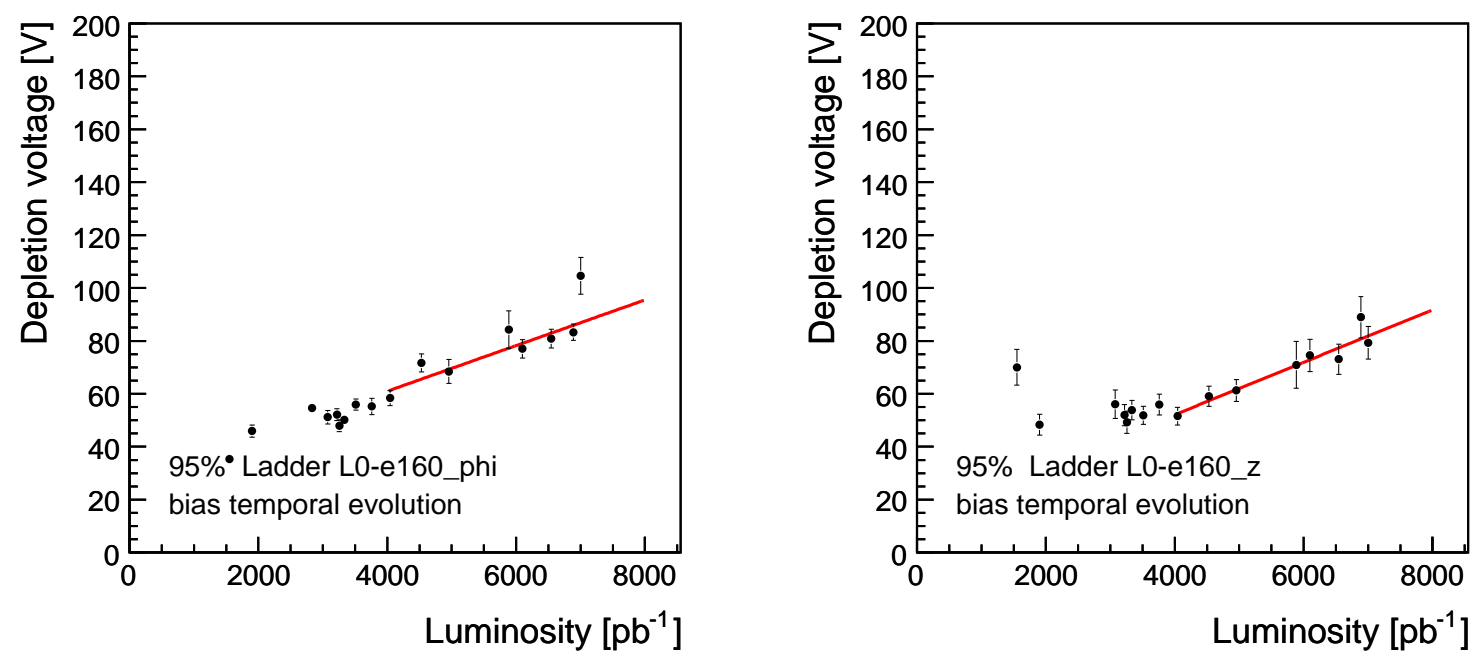

Figure 6.23: Historical evolution of depletion voltage for sensor e160 (luminosity).
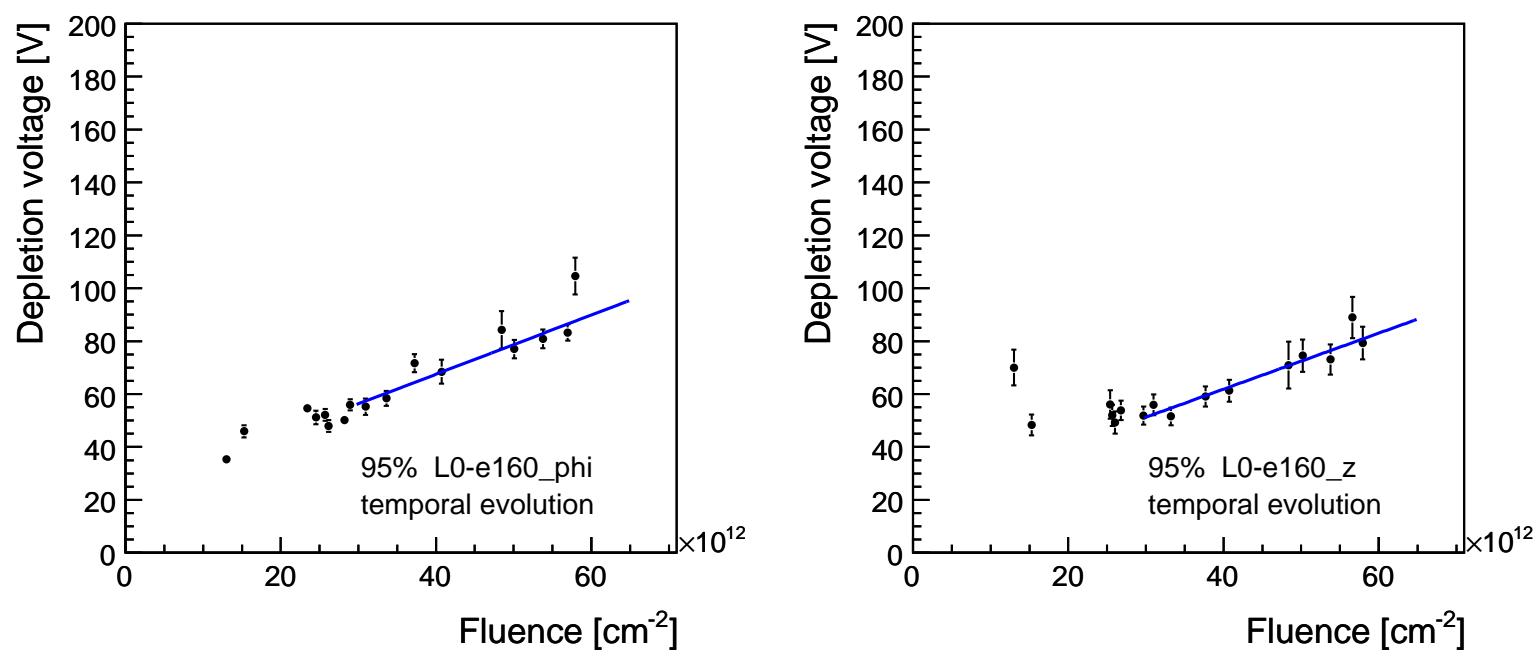

Figure 6.24: Historical evolution of depletion voltage for sensor e160 (fluence). 

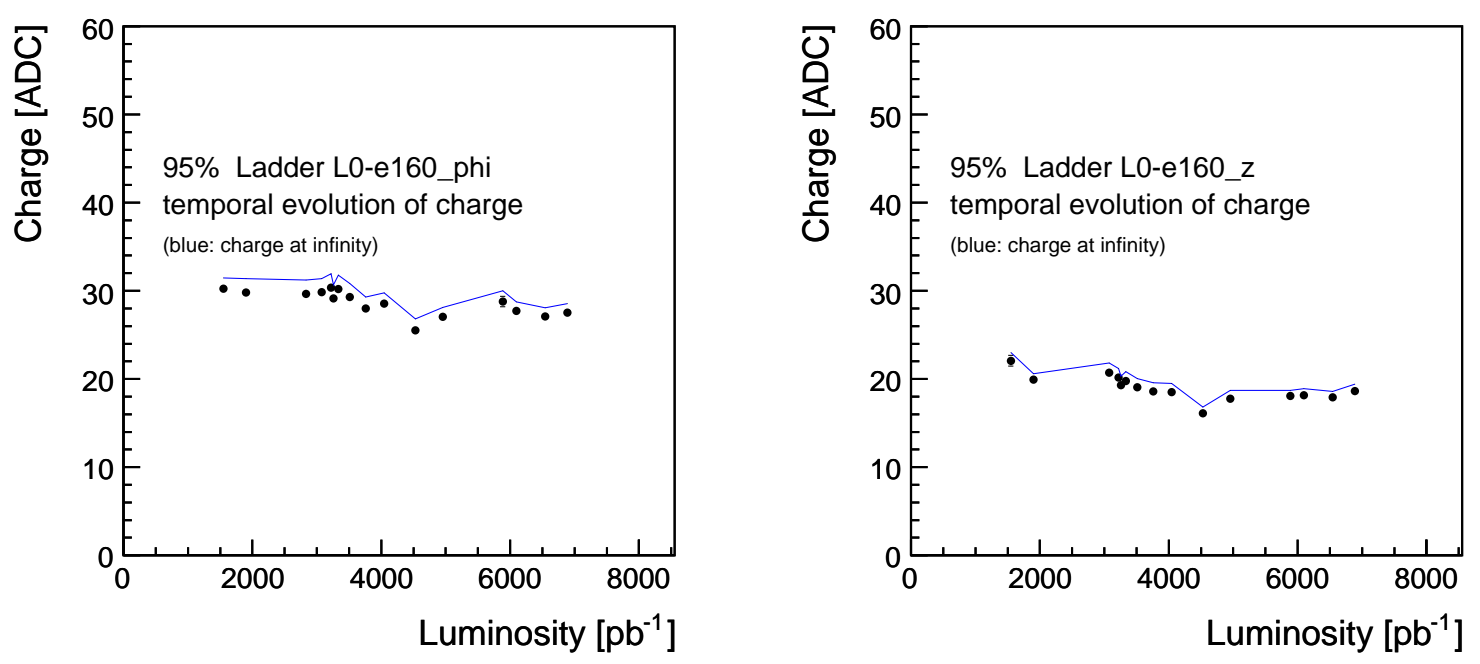

Figure 6.25: Historical evolution of collected charge in full-depletion mode for ladder e160, $\phi$ (left) and $z$ (right) sides.

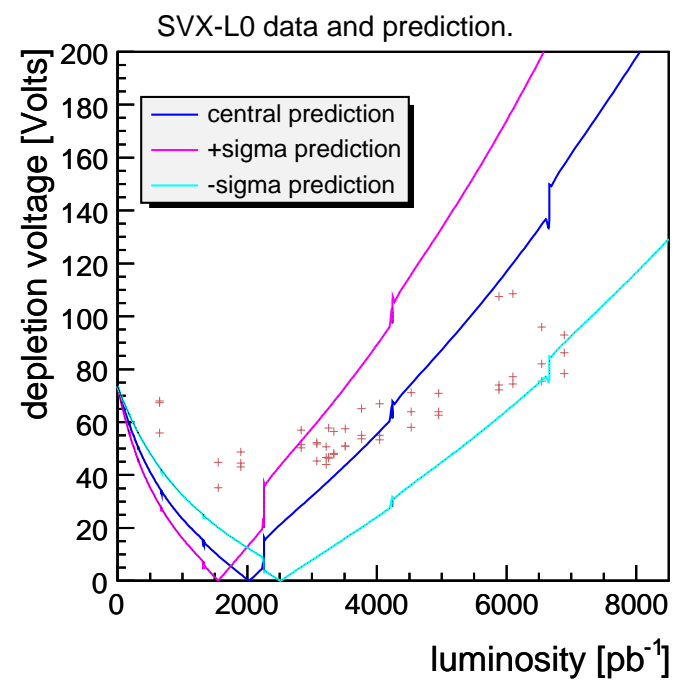

Figure 6.26: SVX-L0 time evolution data over type-inversion prediction model. Dots are the 3 modules for which early signal vs. bias scan data is available. 


\subsubsection{SVX-L0 Extrapolations Summary}

Figure 6.27 compares the extrapolation of the $\phi$ sides of all the 72 sensors of $\mathrm{LO}$ at luminosities of 6888 and $4530 \mathrm{pb}^{-1}$. Figure 6.28 is the equivalent comparison for the $z$ sides. The linear fit performed for the points of more recent luminosity is extrapolated to explore the future behavior and longevity of SVX-LO. The slopes at $4550 \mathrm{pb}^{-1}$ are spread out compared to those at $6888 \mathrm{pb}^{-1}$. This means that at $6888 \mathrm{pb}^{-1}$ the behavior of the sensors is globally more uniform than at $4550 \mathrm{pb}^{-1}$. The general trend in the phi case has been compressed into a depletion voltage interval ranging from 100 to $170 \mathrm{~V}$ at the limit of the extrapolation of $10000 \mathrm{pb}^{-1}$. In the $z$ case we observe a similar evolution with the particularity that at $4550 \mathrm{pb}^{-1}$ there were still sensors with flat and even negative slopes (i.e. sensors not yet inverted) and at latest luminosity of $6888 \mathrm{pb}^{-1}$ the $z$ side has clearly undergone type-inversion.

Figure 6.29 is displayed to compare the global results of SVX-L0 with those of SVX-L1. Both layers are very similar in design, the only difference being the different radial location of each layer. The flat slopes of SVX-L1 evidence a general non-inversion state altough phi side seems to display a general increasing trend.

A global display of data for SVX-LO in terms of fluence is given by Fig. 6.30. This figure shows, for both sides of SVX-LO, the value of the slope of the linear fit made to the fluence points located after the type inversion point. In the figure it can be observed that the slopes are higher for sensors with higher $\phi$ and $z$ cylindrical coordinates (north-east location). 

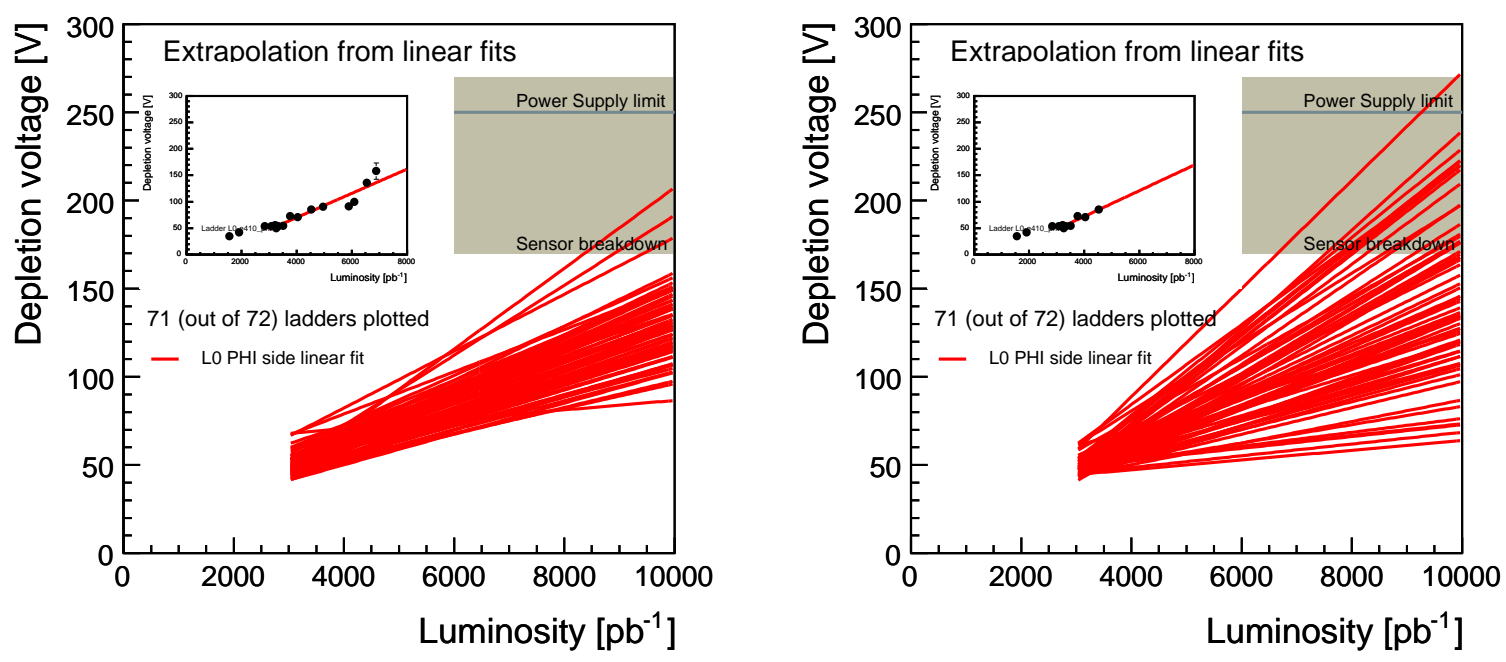

Figure 6.27: Extrapolation fits for all the SVX-L0 sensors' $\phi$ sides at 6888 (left) and $4550 \mathrm{pb}^{-1}$.
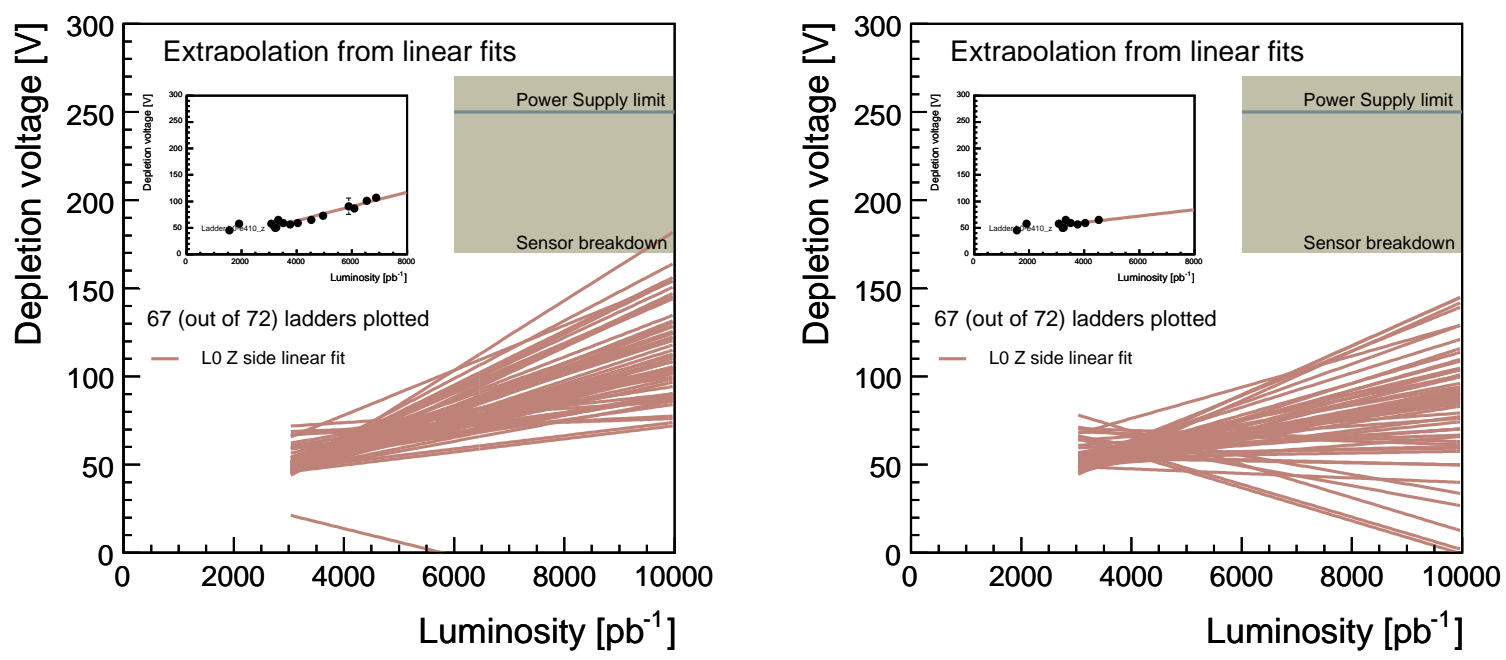

Figure 6.28: Extrapolation fits for all the SVX-L0 sensors' $z$ sides at 6888 (left) and $4550 \mathrm{pb}^{-1}$. 

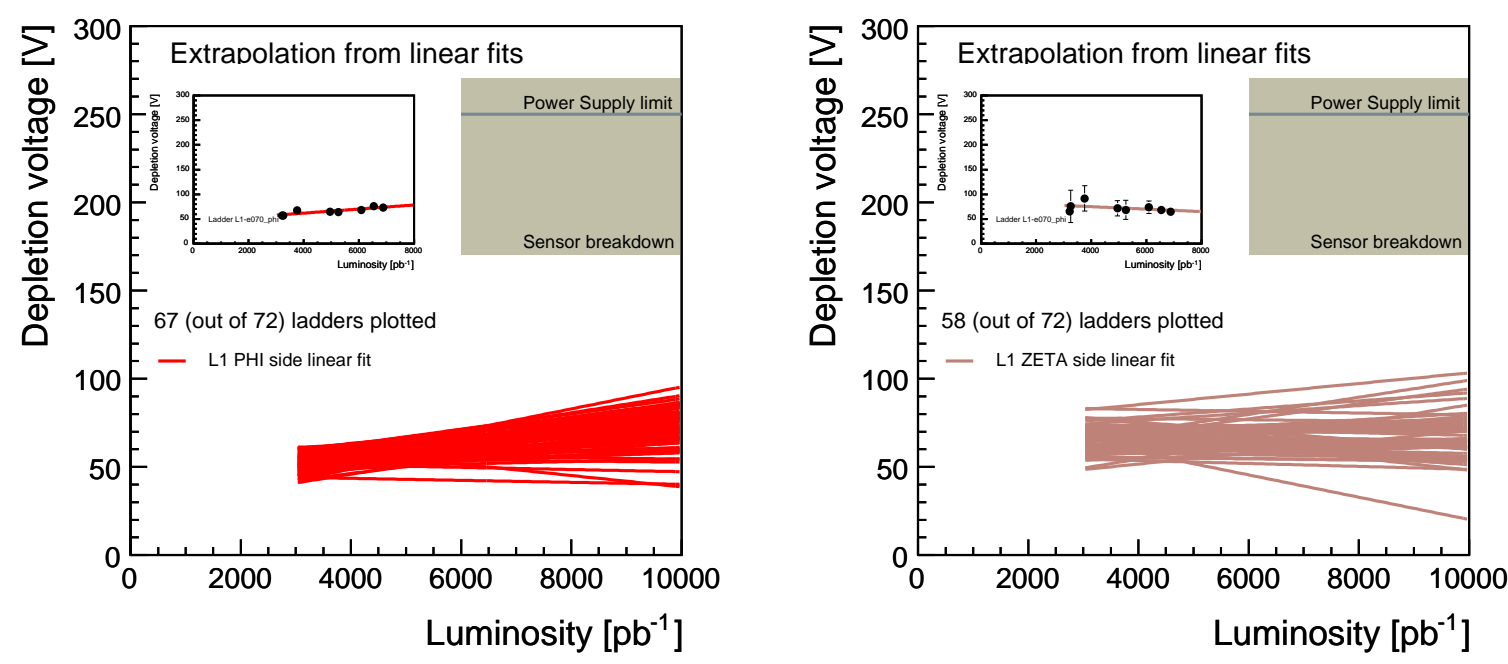

Figure 6.29: Global results for SVX-L1 luminosity plots. Summary of extrapolation fits for all the SVX-L1 sensors' phi sides (left) and $z$ sides (right), at $6888 \mathrm{pb}^{-1}$. Almost flat slopes in SVX-L1 evidence that this layer has not clearly suffered type inversion.

\section{SVX-LO phi}

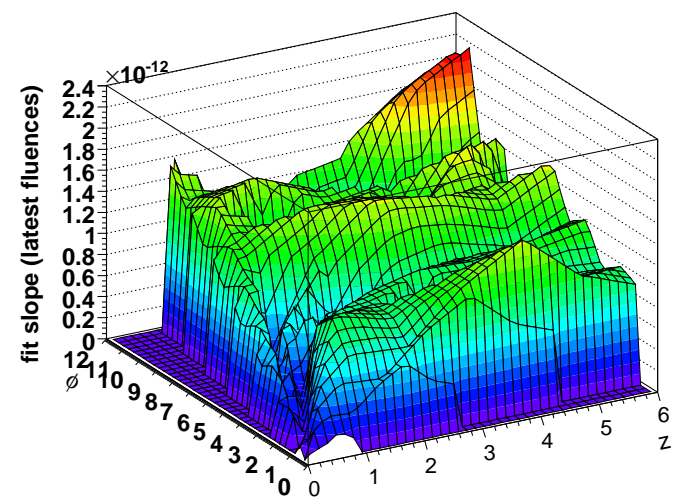

\section{SVX-LO z}

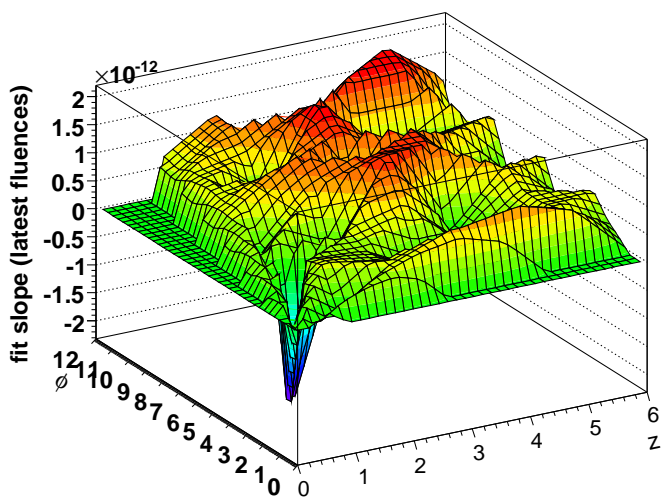

Figure 6.30: Global results for SVX-L0 fluence plots. Summary for SVX-L0 sensors of the linear fits slopes for $p h i$ sides (left) and $z$ sides (right). 


\subsection{Discussion}

This chapter has presented the results of the signal vs. bias scans performed to LOO and SVX-LO at latest integrated luminosity of $6888 \mathrm{pb}^{-1}$.

The signal vs. bias scans show that sensors of both L00 and SVX-LO have suffered typeinversion as a consequence of irradiation (Fig. 6.18). Oxygenated (radiation-hardened) Micron sensors of LO0 delayed this type-inversion phenomenon, whose inversion luminosities are located around $2500 \mathrm{pb}^{-1}$. Standard SGS Thomson and Hamamatsu sensors of L00 displayed inversion luminosities around $1500 \mathrm{pb}^{-1}$ and $1000 \mathrm{pb}^{-1}$, respectively. Hamamatsu sensors of SVX-LO have shown inversion luminosities around $1700 \mathrm{pb}^{-1}$. The difference in inversion luminosities for Hamamatsu sensors of LOO and SVX-LO can be explained by means of the radial dependence of the radiation field [68], [66]. In L00, the fact that the highest luminosity of inversion corresponds to the Micron class of sensors is of importance for the CMS experiment at CERN whose silicon detectors use the same radiation-hardened technology in its sensors.

From the radiation damage viewpoint, the SVX-L0 is a more critical device than L00 since SVX-LO was not specially designed for radiation hardening and its power supply delivery is limited to $250 \mathrm{~V}$. However, the extraplations of the fits of the SVX-LO sensors lie below the safe operation region up to a luminosity of $10000 \mathrm{pb}^{-1}$. This is also true for layer SVX-L1. For this layer in particular the fits are rather flat, a fact that reveals that its sensors have not suffered the inversion process.

The extrapolations of both LOO and SVX-LO have been assumed to be constant but only a further measurement of the rate of change of the depletion voltages after type-inversion will lead to definitive conclusions about the lifetime of the CDF silicon detectors.

For the three classes of sensors of $\mathrm{LOO}$, the depletion voltages after the inversion point display an increasingly linear behavior, meaning that after inversion the creation of new negative centers in the space charge is also linear. The extrapolations of the linear fits made to those points are useful to predict the global future performance of LO0. All those fit extrapolations lie below the power supply limit of $500 \mathrm{~V}$ and the sensor breakdown region. With the actual results we can conclude that the sensors will be able to be depleted at least up to a luminosity of $10000 \mathrm{pb}^{-1}$.

The silicon detectors are the most critical subsystem of the CDF experiment. In particular, the functionality of its innermost layers, LOO and SVX-LO, is essential for the completion of the physics program of CDF. The political discussion concerning the extension of the Tevatron Run II to 2010-2011 is being based on the L00 and SVX-LO extrapolations plots and studies shown in this document. 
One of the main questions derived from the CDF type inversion data is the realtive mismatch between the modelization and the experimental data, as shown in Fig. 6.26 and in 6.31.
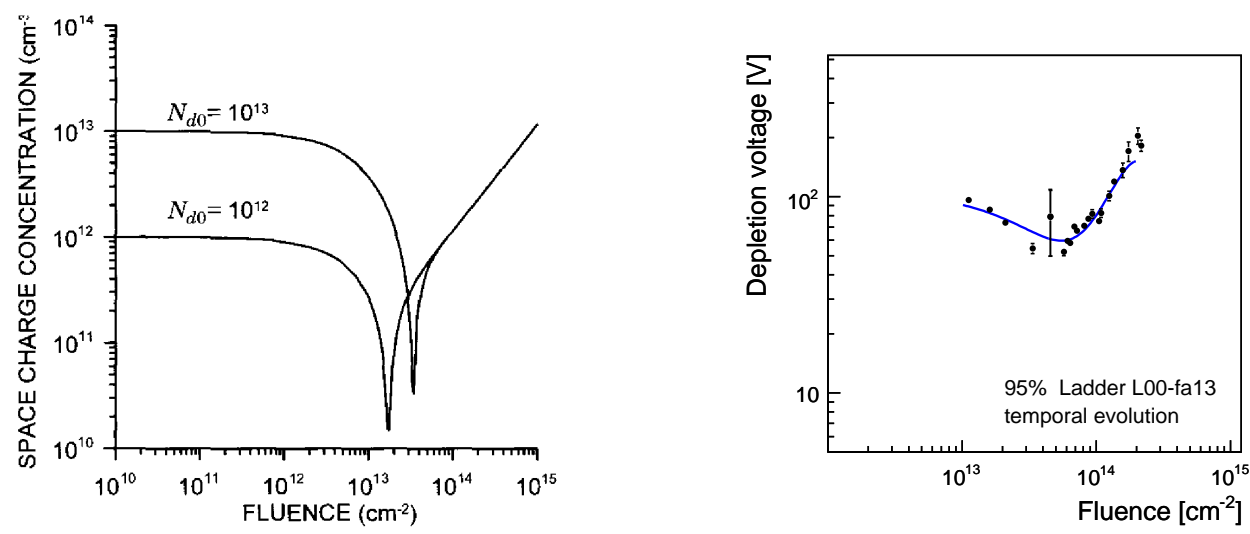

Figure 6.31: Left: calculated space charge vs. high-energy proton fluence for silicon with initial donor concentrations $N_{d 0}$ of $10^{12}$ and $10^{13} \mathrm{~cm}^{-3}$. Whith an infinitely fine calculation grid both distributions would dip to zero (Ref. [70]). Right: experimental data of the inversion curve from CDF (L00 module fa13). The fact that the minimum of this curve does not reach to zero volts is in conflict with the model.

While the depletion voltage drops to zero in the model, the experimental data show a curve with a minimum located in the range of 35 to 50 volts. Assuming in principle the validity of the model we should explain the voltage shift displayed by the data. Explanations could range from technical voltage drops to unexpected effects in the interaction of radiation with matter. However this was studied in detail in chaper 5 limiting this effect to less than $7.5 \mathrm{~V}$, too small to explain the mismatch displayed by 6.31 , so the hypothesis of a voltage drop is then discarded. Another possible explanation would be the nature of the doping process inside the sensor, a topic that would be dealt in Chapter 7. 


\section{Chapter 7}

\section{Description of radiation aging effects in LOO and SVX-II detectors}

\subsection{Monitoring of detector parameters}

To illustrate the radiation-aging in the L00 and SVX systems we focus on the behavior and evolution of four important parameters of the detector:

- Efficiency

- Collected Charge or Signal

- Resolution

- Cluster Size

These parameters were measured using the hits on track already discussed in the depletion voltage measurement. Parameter efficiency is a relative efficiency of the sensor under test with respect of the rest of the silicon detector. The collected charge is the quantity of electrical charge released after ionization by radiation of the semiconductor bulk and collected by the readout electrodes. The RMS of the distribution of residuals (Fig. 7.1, left) between the extrapolated track and the meassured hit position is taken as a measure of the position resolution. Cluster size (Fig. 7.1, right) is the number of strips activated (i.e. reading signal above noise) by a track hit. In section 7.3 we also investigate the behaviour of the derivative of the signal with respect to the bias voltage as it provides insight on the doping profile in the bulk as discussed in appendix $D$. 

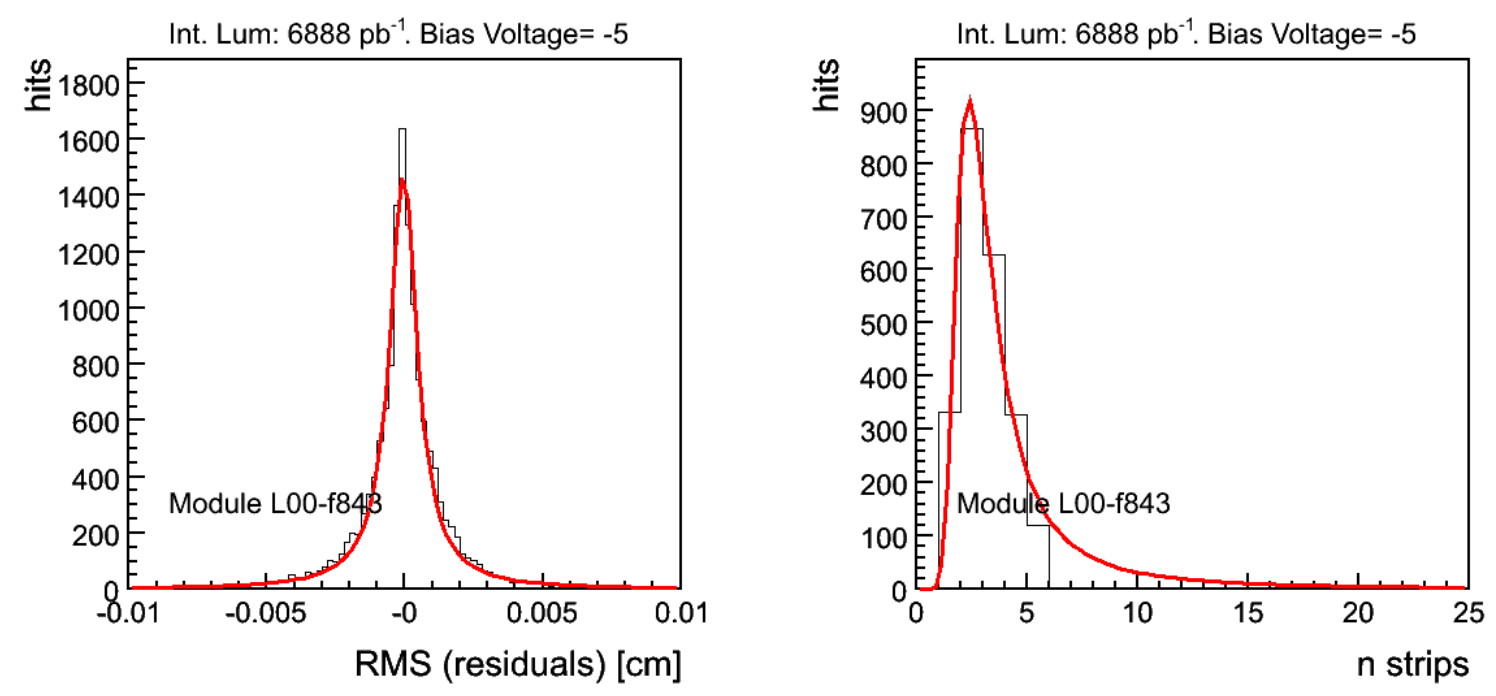

Figure 7.1: Example of RMS and cluster size (number of strips) distributions and fitting functions at bias voltage of -5 volts with respect to the nominal for L00 sensor f843. The Lorentzian (on the the residuals distributions) and Landau (on the cluster fits distribution) fits are plotted to guide the eye.
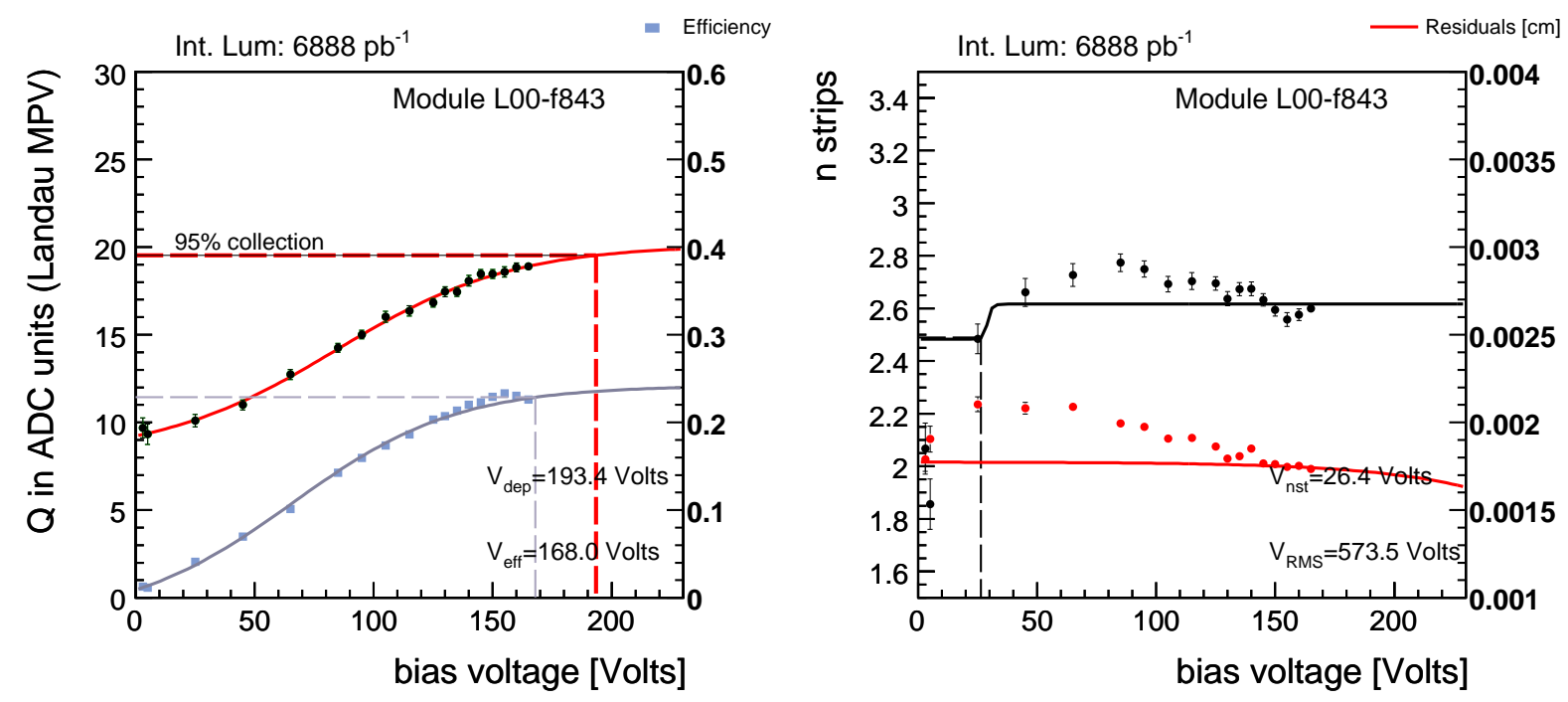

Figure 7.2: Four detector variables as a function of bias voltage for L00 sensor f843. The fits on the right plot did not converge for this particular detector. 


\subsubsection{Aging of $\mathrm{LOO}$}

For all the LO0 sensors, the efficiency shows a general decreasing pattern along luminosity (Fig. 7.3, left plot, red graph). The values of the efficiency plot are systematically higher for the wide sensors. At later luminoisities the collected charge and efficiency recover mildly as the nominal voltage was systematically increased as a mitigation policy against aging.

The charge collected by the sensors (signal) seems constant along luminosity for all the L00 sensors (Fig. 7.3, left plot, blue and black graphs). There are two different measurements of the collected charge displayed: the first one, in black dots, shows the charge corresponding to the depletion voltage value ( $95 \%$ of collection charge) of the fitting sigmoid. The second one, in a continuous blue line, corresponds to the saturation of the sigmoid (100 $\%$ of the collection charge, or the value of the charge at infinite voltage). To check the quality of the sigmoid fitting-function both data plots should not be far away from each other.

The resolution and cluster size plots display very similar curves, the cluster size for L00 narrow sensors being slightly lower than for wides (Fig. 7.3, right plot). In the case of the cluster size, it can vary from 2.8 to 3.3 stripes along luminosity. Hence we would assume the cluster size to be constant and with a practical value of 3 stripes. There is an overall trend of wider clusters and degraded position resolution as luminosity increases.

\subsubsection{Aging of SVX-LO}

Graph of charge vs luminosity plot is slightly decreasing for both LO-phi and L0-z (Fig. 7.4). Efficiency vs luminosity plot has an interesting evolution along z-axis sensors (for both phi and $z$ sides): the curve maintains its shape but rises upward with $\eta$, reaching a maximum at $\eta=90$ (collision point), and then simmetrically decreases (Table 7.1).

Absolute values are larger for the phi side (Fig. 7.4). Resolution vs luminosity plot is constant for both LO-phi and LO-z sides (Fig. 7.5). Cluster size vs luminosity plot is interestingly increasing only for L0-phi side, remaining constant for the $\mathrm{z}$ side (Fig. 7.5). This effect would be expected after inversion, since the lack of " $n$-stops" on the p side would reduce the interstrip resistance.

\subsubsection{Aging of SVX-L1}

Charge vs luminosity plot is slightly decreasing or constant for phi and z sides of SVX-L1 (Fig. 7.6). Efficiency vs. luminosity plot displays a similar evolution along z-axis than that showed by LO (for both L1-phi and L1-z sides). Also, absolute values are larger for the L1-phi side (Fig. 7.6). Resolution vs. luminosity plot is quite constant for L1-phi and L1-z 

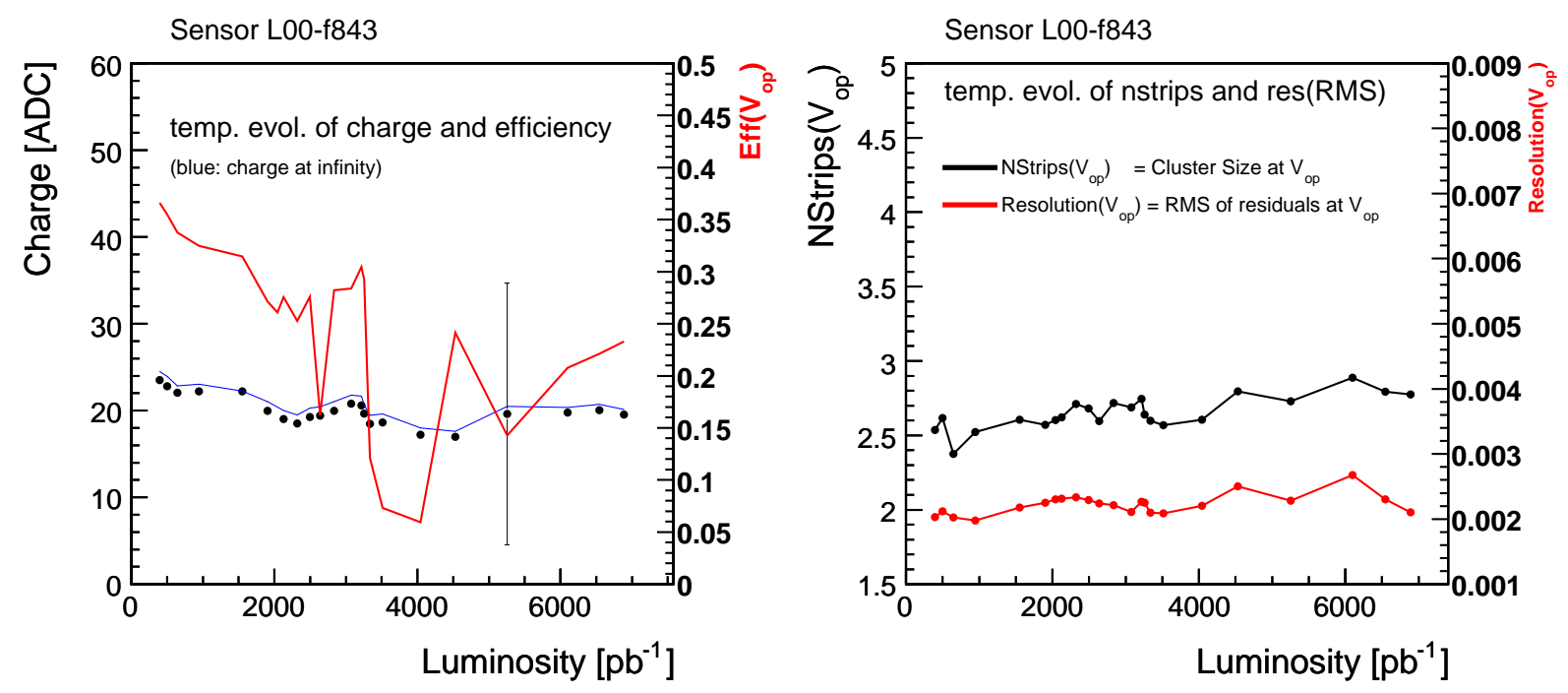

Figure 7.3: Evolution of the four detector variables for L00 sensor f843.

sides (Fig. 7.7). Cluster size vs luminosity plot also increases only for the L1-phi side, and is almost constant or with decreasing trend for its $\mathrm{z}$ side (Fig. 7.7). 

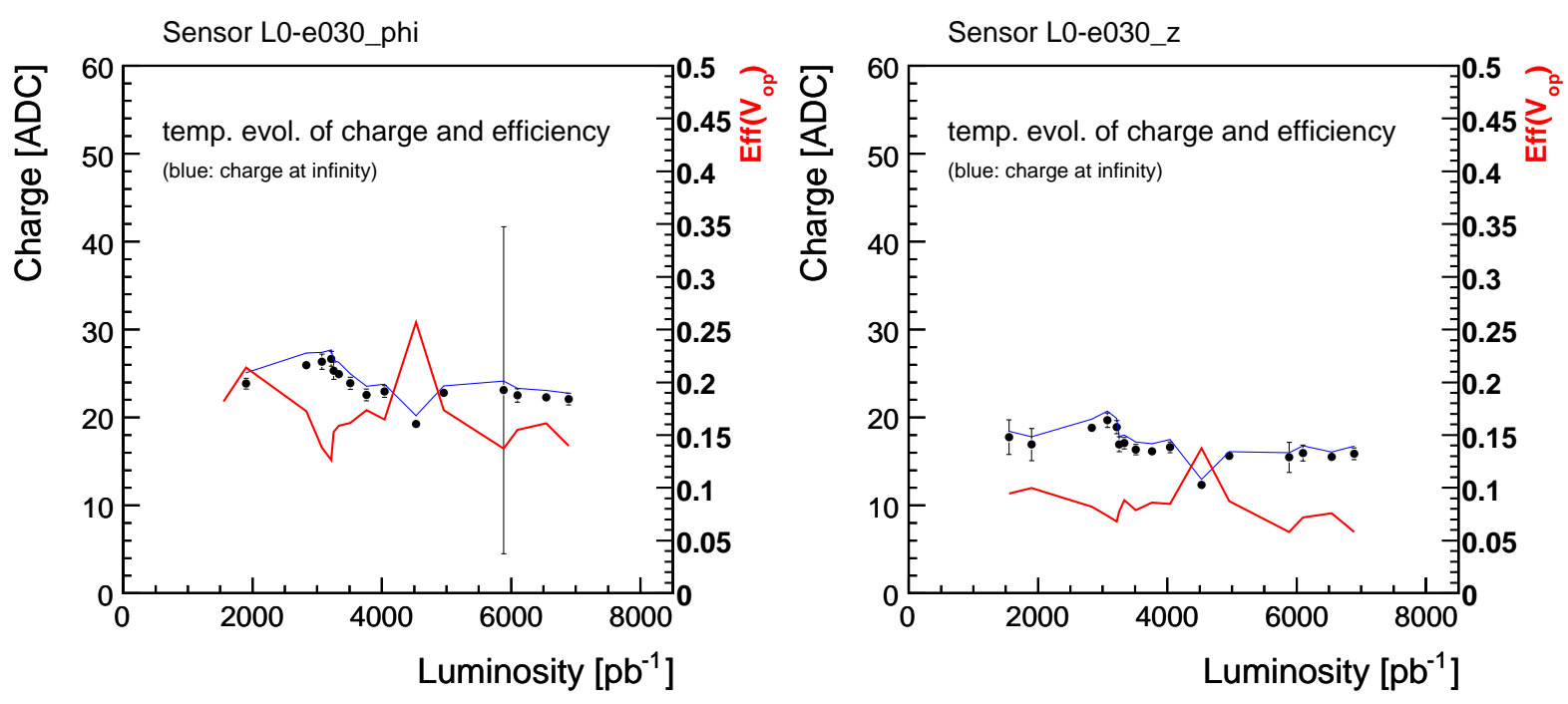

Figure 7.4: Historical plot of charge and efficiency for SVX-L0 sensor e030, phi (left) and z sides.
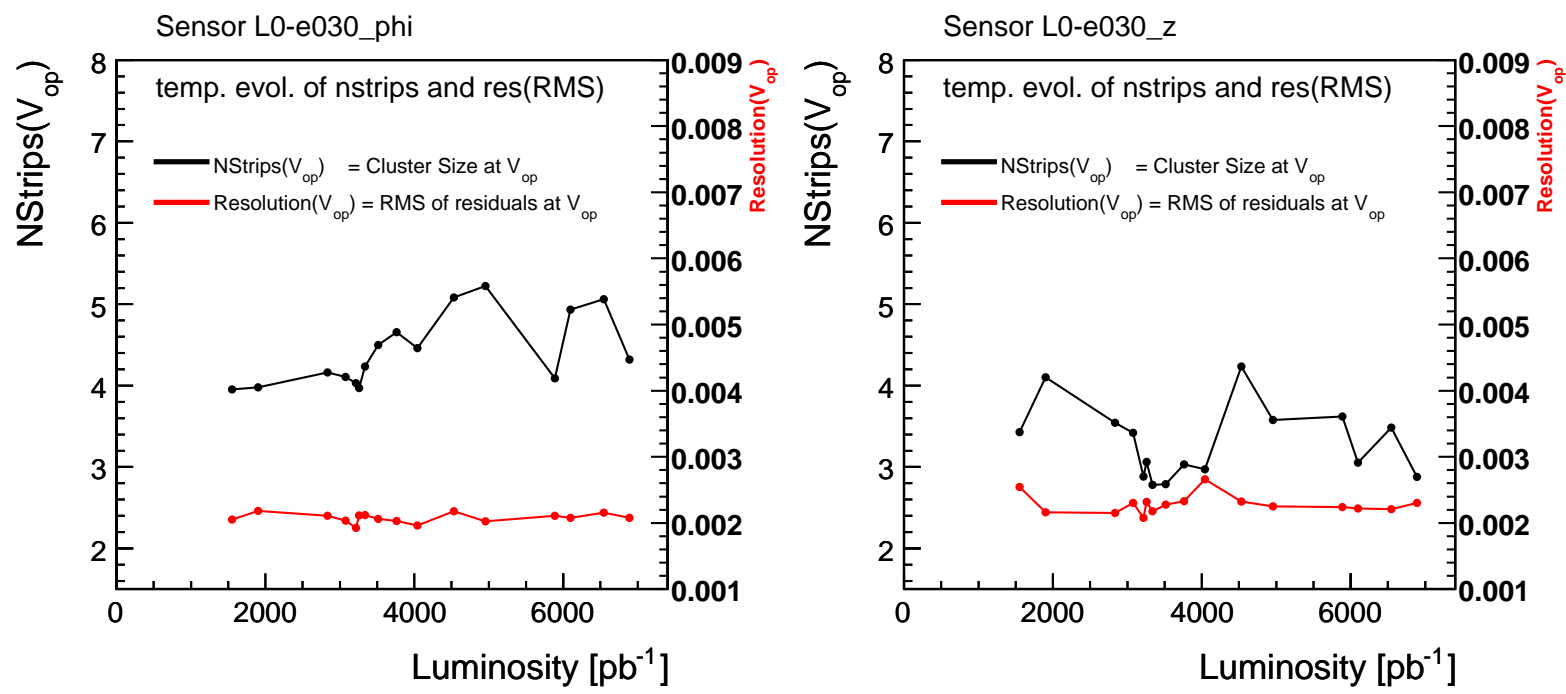

Figure 7.5: Historical plot of cluster size and resolution for SVX-L0 sensor e030, phi (left) and z sides. 

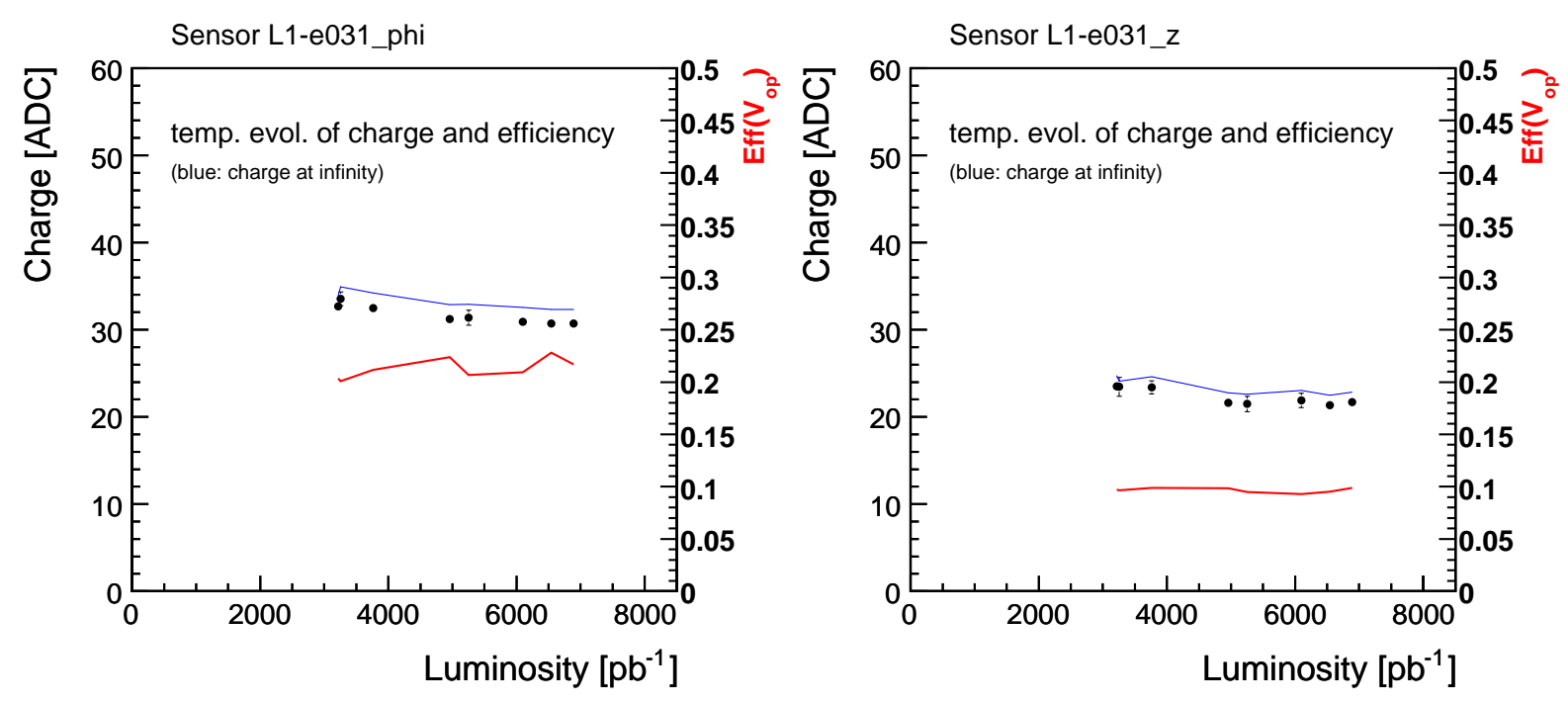

Figure 7.6: Historical plot of charge and efficiency for SVX-L1 sensor e031, phi (left) and z sides.
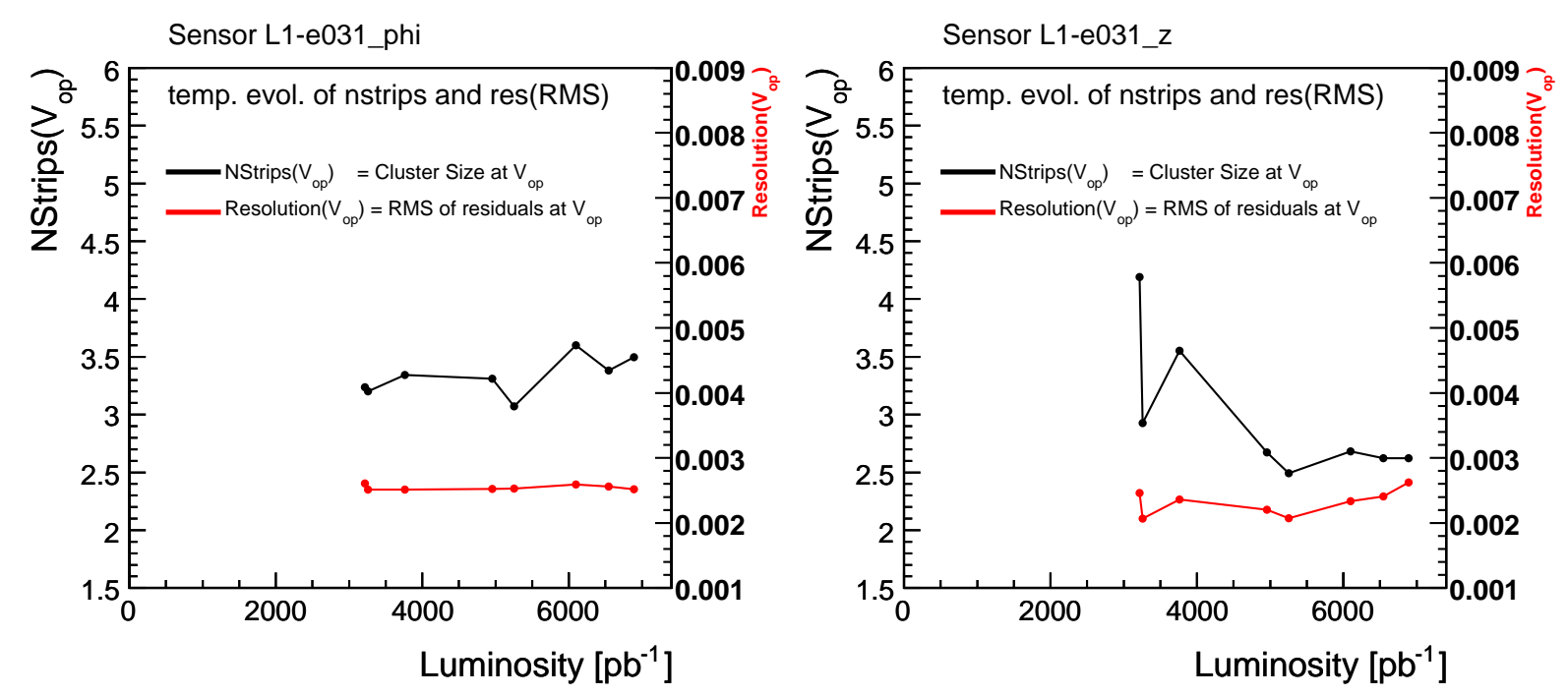

Figure 7.7: Historical plot of cluster size and resolution for SVX-L1 sensor e031, phi (left) and z sides. 


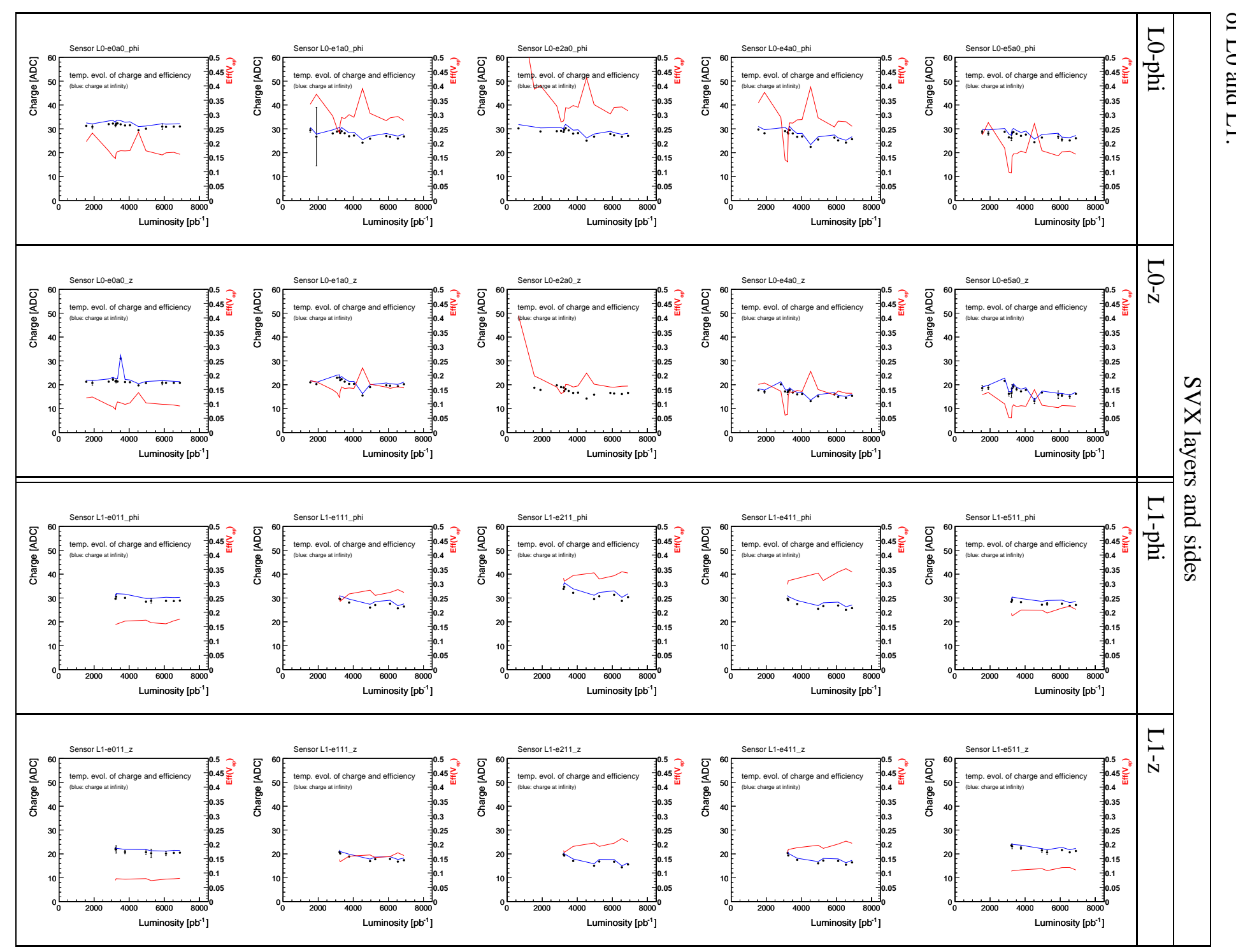

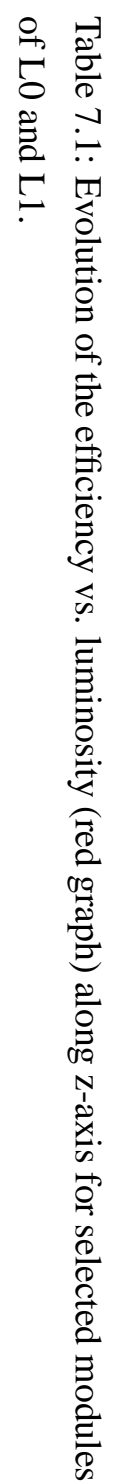




\subsection{Summary of parameters by layer}

To summarize the values of the reference parameters of the detector, Table 7.2 shows their evolution as a function of luminosity averaged over modules in L00, SVX-LO $\phi$ and SVX-LO $z$.

Table 7.2: Global values per layer. Average over total number of sensors of detector's parameters versus luminosity.

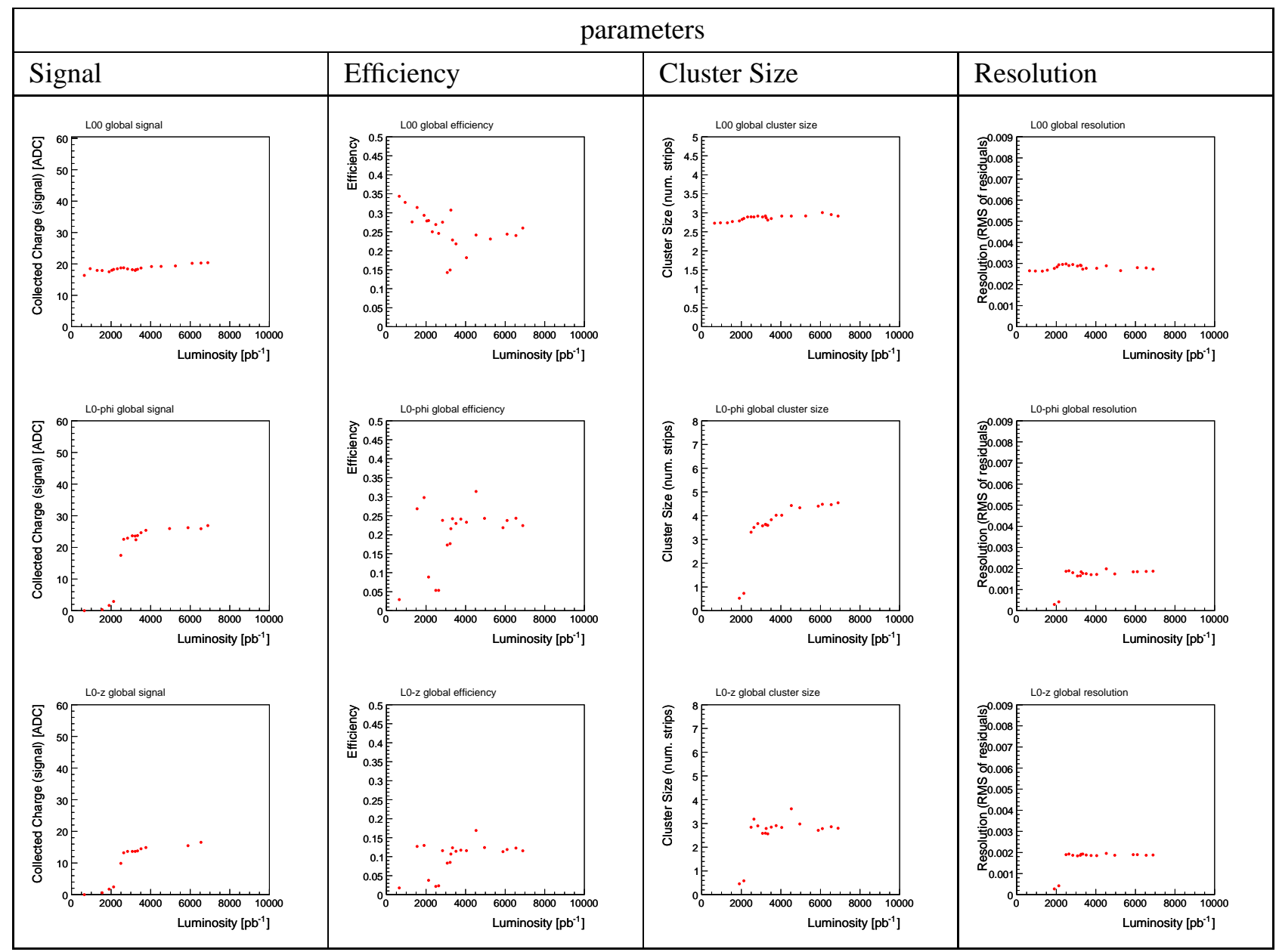




\subsection{Behavior at low bias voltage}

\subsubsection{L00}

Sensors f850 (Hamamatsu), f843 (SGS Thomson) and f813 (Micron) have been designed as representatives of the $\mathrm{LOO}$ sensors. For each of those sensors it has been selected a set of three points of luminosity (irradiation stages):

- luminosity far before type-inversion

- luminosity at the type-inversion point.

- luminosity far after type-inversion

For every luminosity point and sensor class the different parameters of the sensor were displayed: collected charge, first derivative of collected charge, efficiency, cluster size (number of stripes), and resolution (RMS of residuals). Tables 7.3, 7.4, and 7.5 summarize the plots. Tables 7.7 and 7.8 shows the behavior at low bias voltage for the complete set of luminosity points.

An interesting observation arises related to the first derivative of the collected charge: for the lowest luminosity -before inversion- the value of the peak is 0.4. At the inversion point it has increased to 0.8 and finally, at the current luminosity, moves forward and to 0.1. This pattern can be explainable from the viewpoint of a sensor slightly underdepleted with time. However it is interesting to plot the first derivative of a sigmoid fit fitting only the low-voltage points. This is displayed in Table 7.5 where in its first row it has been plotted the red an black sigmoids for full range and low range of voltages, respectively, and in its second row their first derivative with the same color code.

Another observation can be extracted from Tables 7.7 and 7.8: the slope of the lowvoltage fit (black plot, first derivative of sigmoid fit for low voltages) increases between $394 \mathrm{pb}^{-1}$ and $2127 \mathrm{pb}^{-1}$ (this is expected since the depletion voltage decreases). In $2127 \mathrm{pb}^{-1}$ there is a transition in this behavior since from this luminosity point the value of the slope does not increase anymore and remains constant; the slope at low bias voltages is independent of the applied bias voltage.

A summarization for LO0 of the previous observation about Tables 7.7 and 7.8 is given by plots in Table 7.17. 
Table 7.3: Type-inversion evolution for L00-f850, example of Hamamatsu class sensor (wide). Inversion point at $944 \mathrm{pb}^{-1}$. First row contains the turn-on curves of the collected charge and efficiency set of points. In the second row is displayed the first derivative of the red sigmoid fitting the charge points of first row. Third row is reserved for the number of strips and the residuals.

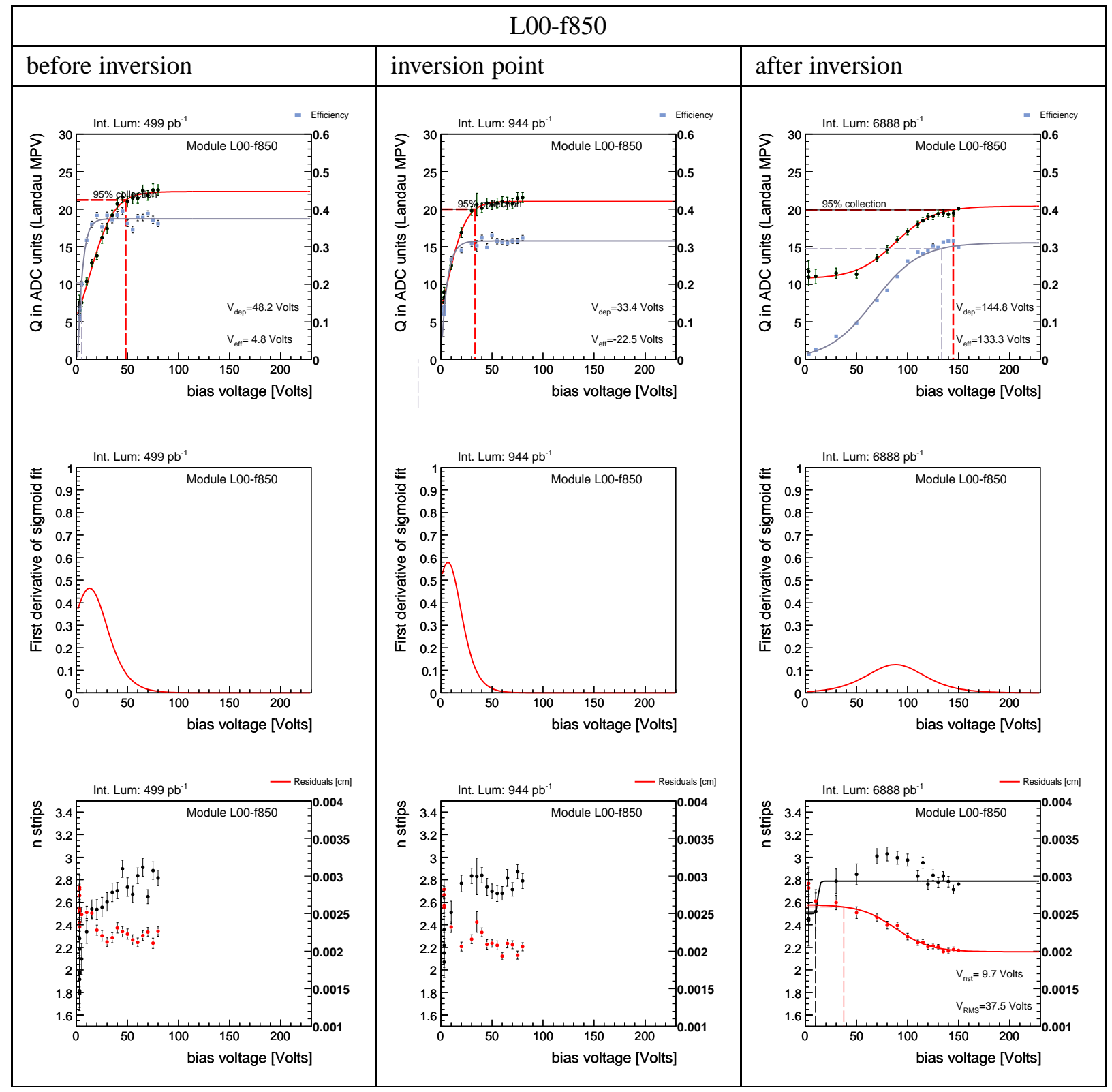


Table 7.4: Type-inversion evolution for L00-f843, example of SGS Thomson class sensor (standard narrow). Inversion point at $2041 \mathrm{pb}^{-1}$. First row contains the turn-on curves of the collected charge and efficiency set of points. In the second row is displayed the first derivative of the red sigmoid fitting the charge points of first row. Third row is reserved for the number of strips and the residuals.

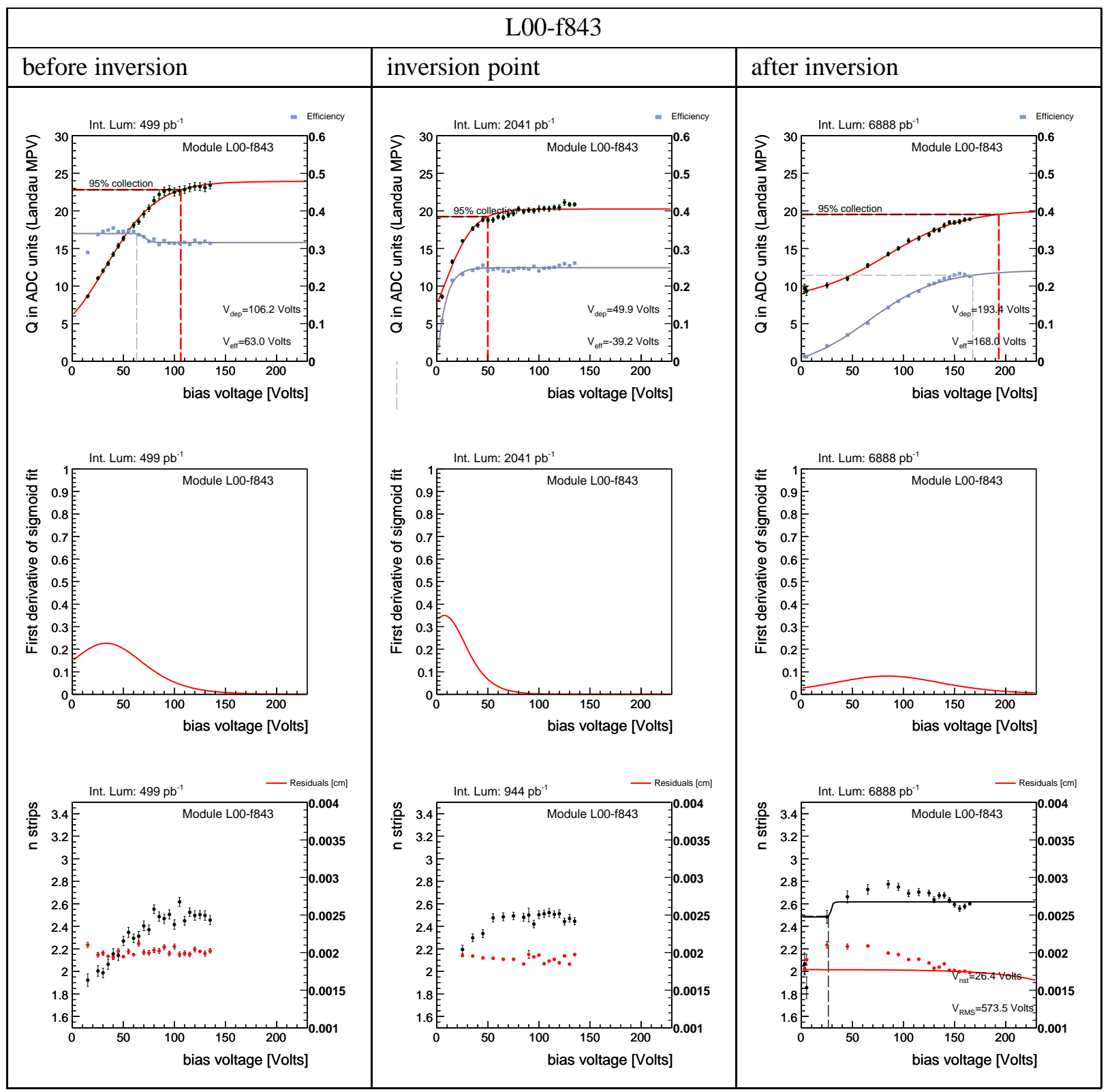


Table 7.5: Type-inversion evolution for L00-f813, example of Micron radiation-hardened class sensor (oxygenated narrow). Inversion point at $2494 \mathrm{pb}^{-1}$. First row contains the turn-on curves of the collected charge and efficiency set of points. In the second row is displayed the first derivative of the red sigmoid fitting the charge points of first row, and an analogous black curve for the black sigmoid curve that fits the region of low voltages in the first row. Third row is reserved for the number of strips and the residuals.

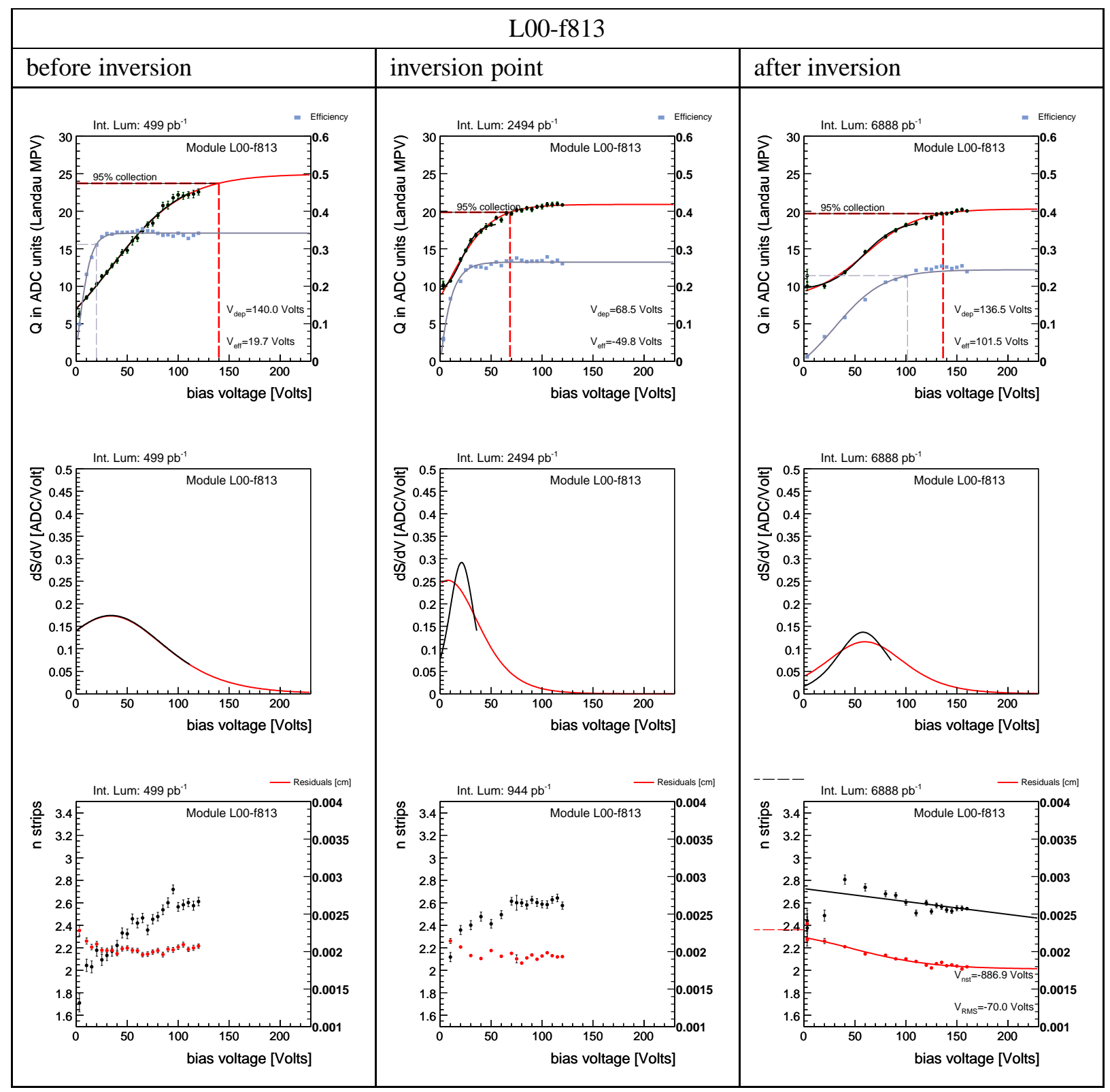


Table 7.6: Collected charge, efficiency, cluster size and residuals evolution for the three classes of L00 sensors.

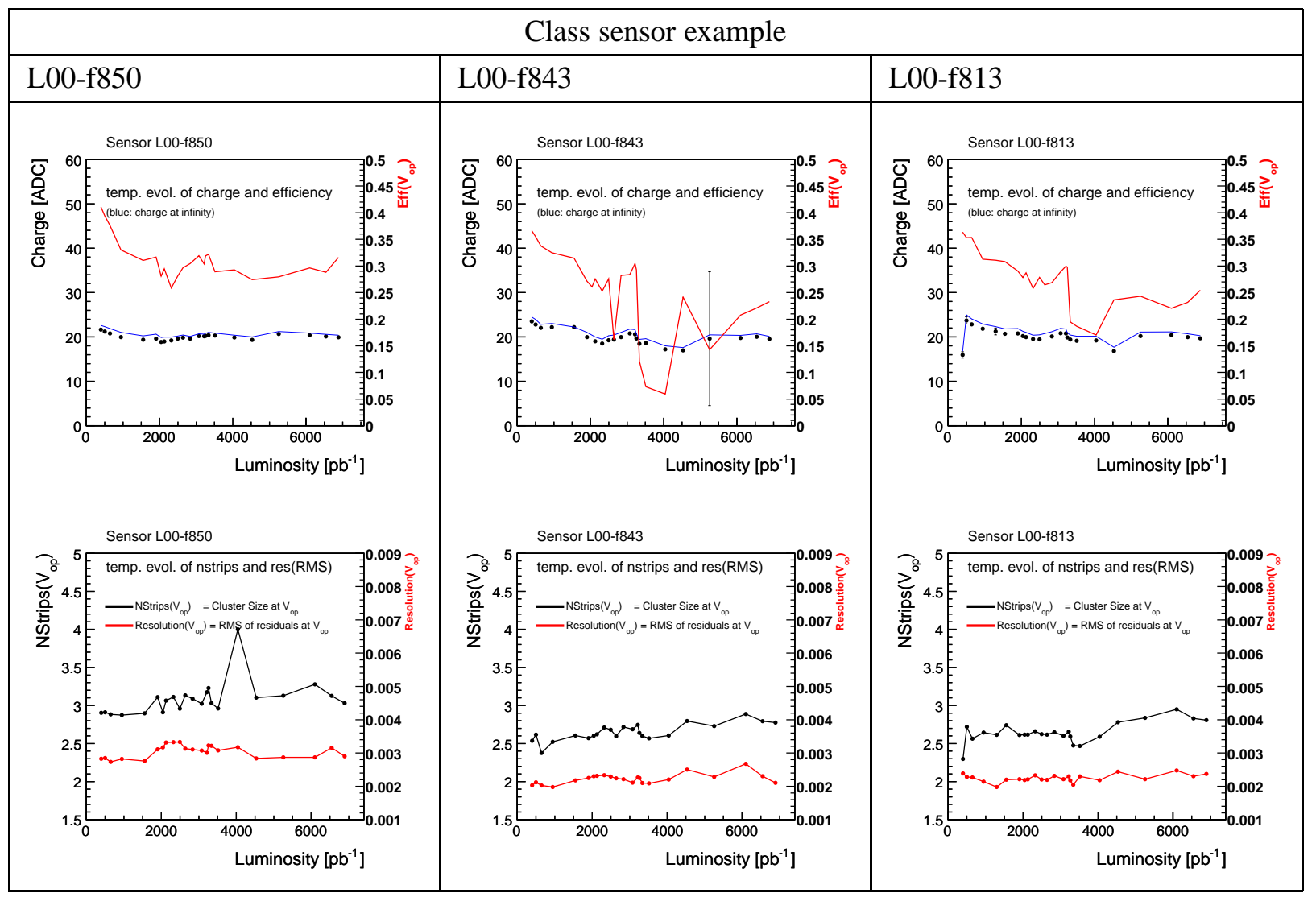


Table 7.7: Type-inversion evolution for L00-f813, example of Micron radiation-hardened class sensor (oxygenated narrow). Inversion point at $2494 \mathrm{pb}^{-1}$. All luminosities part I. Black plot is a fit for low bias voltages (limit of the black fit: $80 \% V_{d} e p$ ).

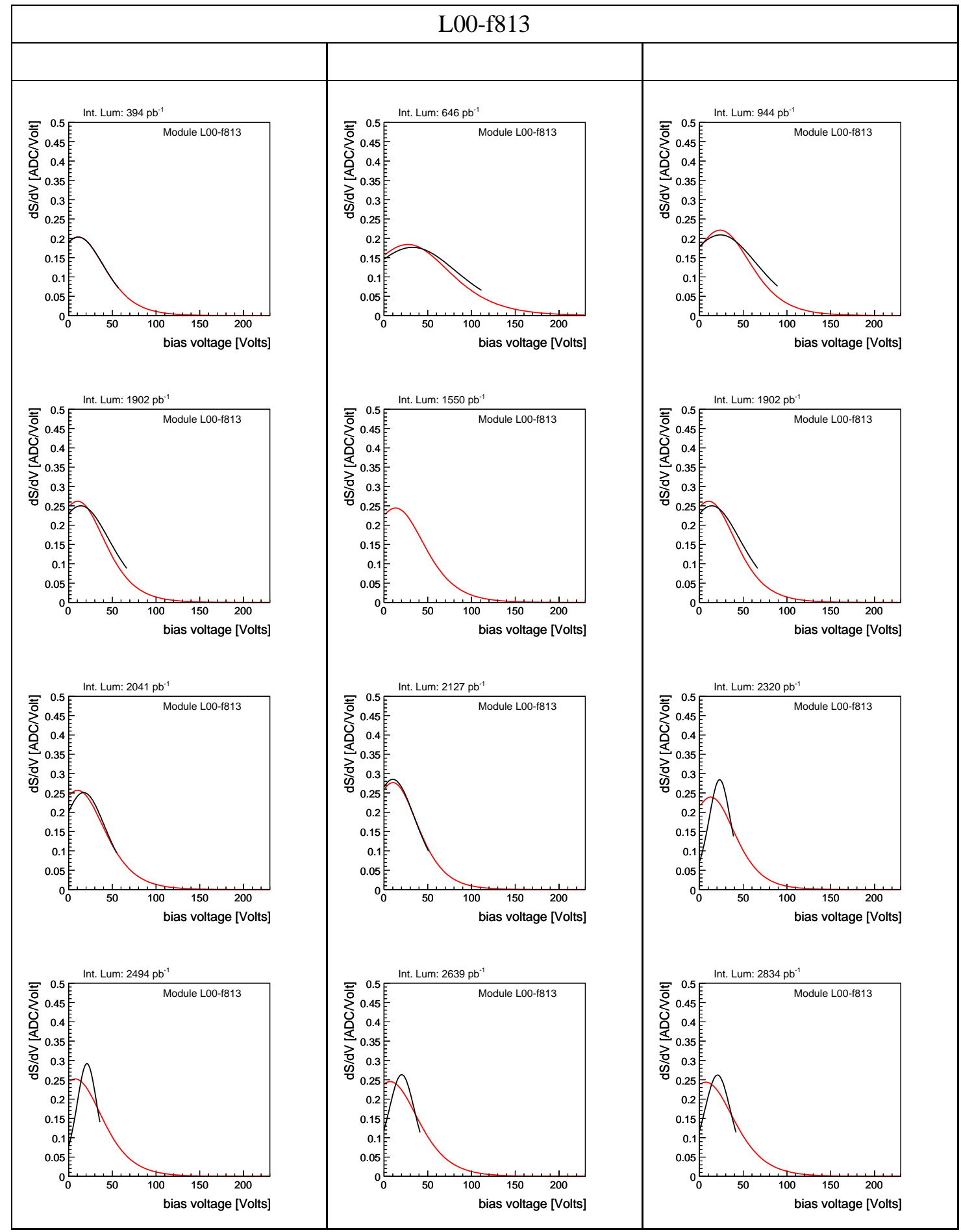


Table 7.8: Type-inversion evolution for L00-f813, example of Micron radiation-hardened class sensor (oxygenated narrow). Inversion point at $2494 \mathrm{pb}^{-1}$. All luminosities part II. Black plot is a fit for low bias voltages (limit of the black fit: $80 \% V_{d} e p$ ).

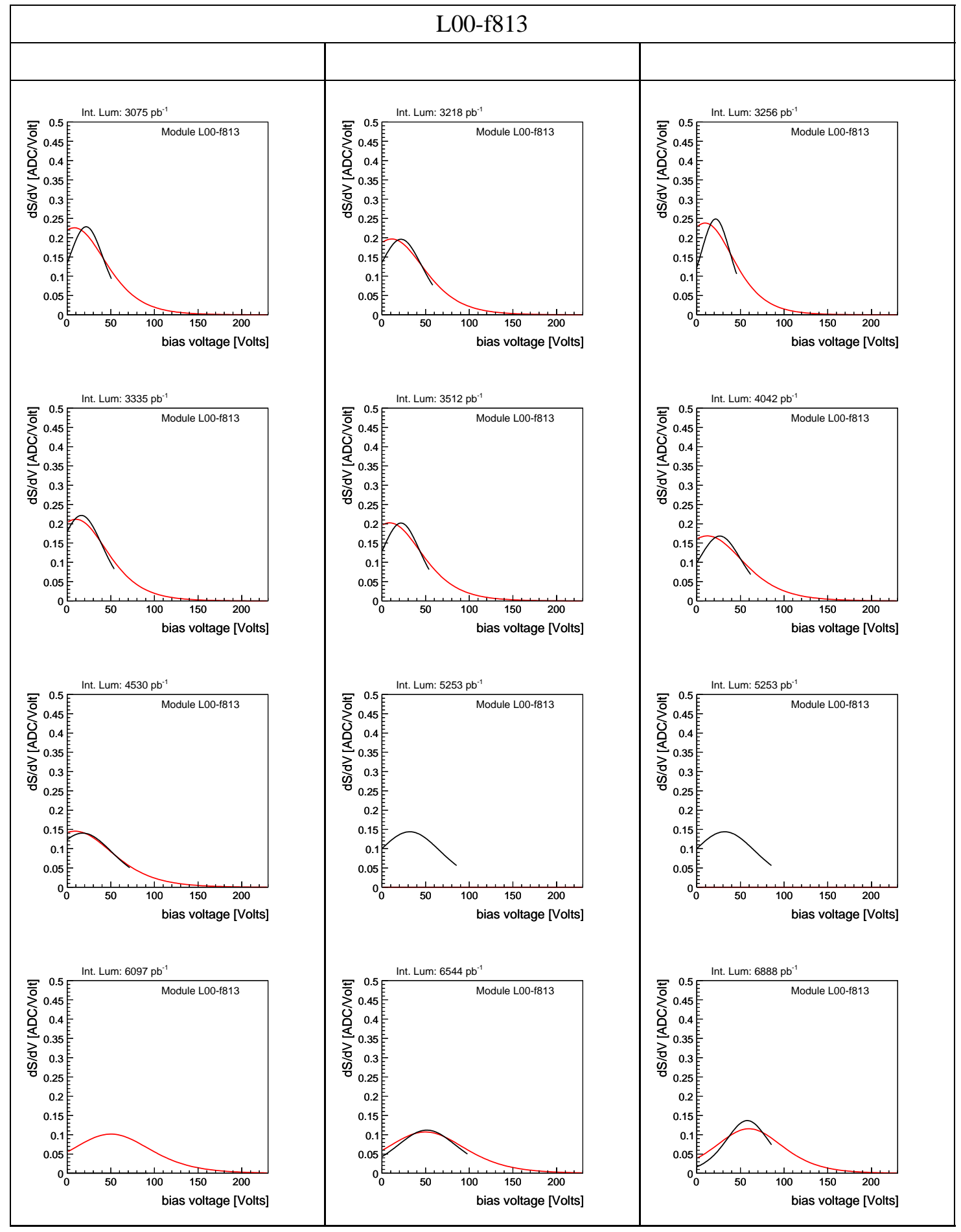




\subsubsection{SVX-LO}

Sensors e090, e140, and e2a0 are the devices with more statistics on SVX-L0. Similarly as in the case of $\mathrm{LOO}$, for each of those three sensors it has been selected a set of three points of luminosity (irradiation stages):

- luminosity far before type-inversion

- luminosity at the type-inversion point.

- luminosity far after type-inversion

For every luminosity point and sensor class the different parameters of the sensor were displayed: collected charge, first derivative of collected charge, efficiency, cluster size (number of stripes), and resolution (RMS of residuals). Tables 7.9, 7.10, 7.11, 7.12, 7.13, and 7.14 summarize the plots.

A general display of the SVX-LO low-voltage behavior dealt in the previous Tables is given by plots contained in Table 7.18. 
Table 7.9: Type-inversion evolution for L0-e090, a Hamamatsu class sensor. Inversion point at $1902 \mathrm{pb}^{-1}$. Each one of the three columns represents a stage point in the lifespan of the sensor ( $p h i$ and $z$ sides are shown): before, after and at inversion. The red sigmoid fits the whole set of charge points while the black sigmoid fits only the subset of points at low voltages. Second row holds the first derivative of those curves, respecting the color code.

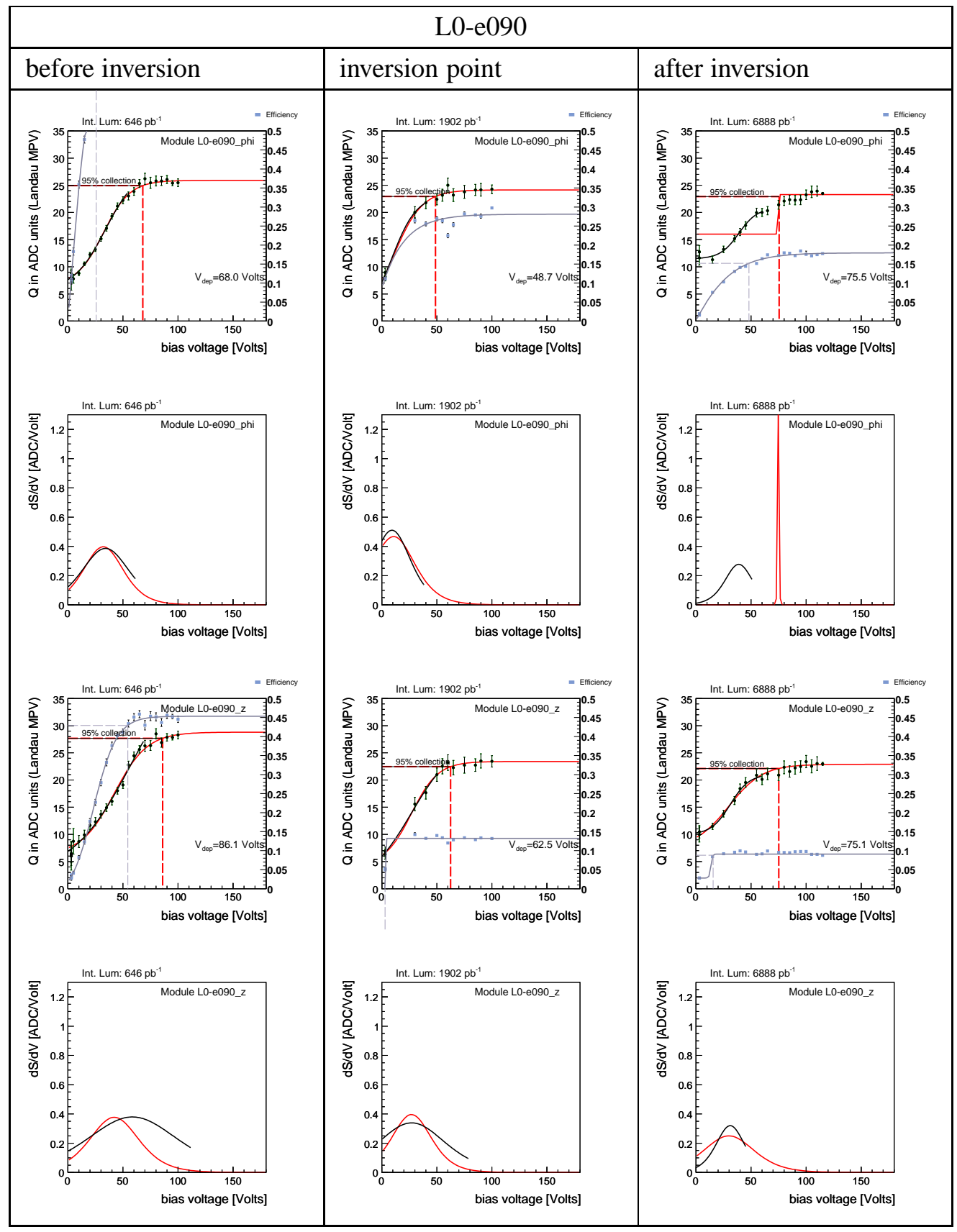


Table 7.10: Type-inversion evolution of cluster size and residuals for L0-e090, a Hamamatsu class sensor. Inversion point at $1902 \mathrm{pb}^{-1}$.

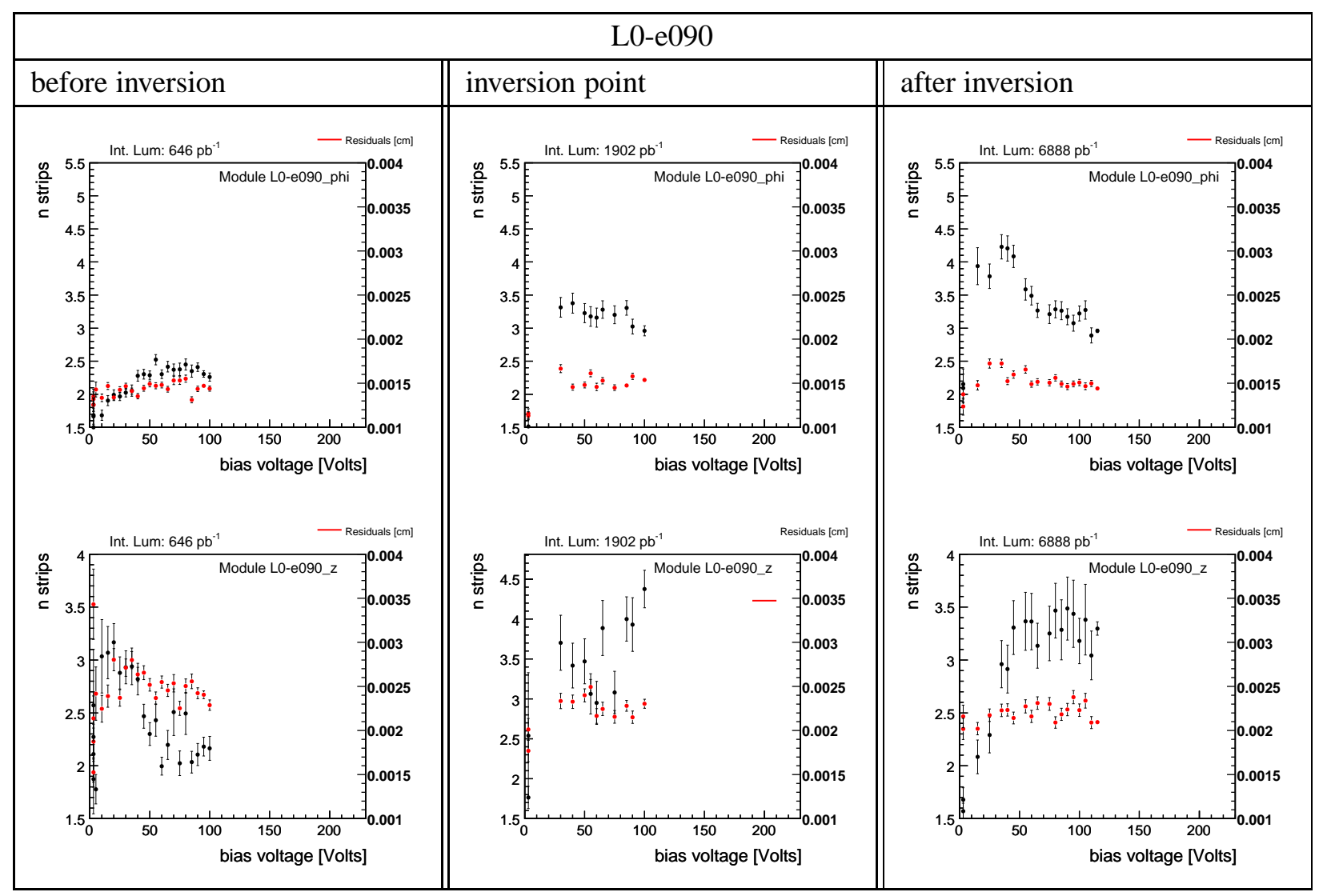


Table 7.11: Type-inversion evolution for L0-e140, a Hamamatsu class sensor. Inversion point at $1902 \mathrm{pb}^{-1}$. Each one of the three columns represents a stage point in the lifespan of the sensor (phi and $z$ sides are shown): before, after and at inversion. The red sigmoid fits the whole set of charge points while the black sigmoid fits only the subset of points at low voltages. Second row holds the first derivative of those curves, respecting the color code.

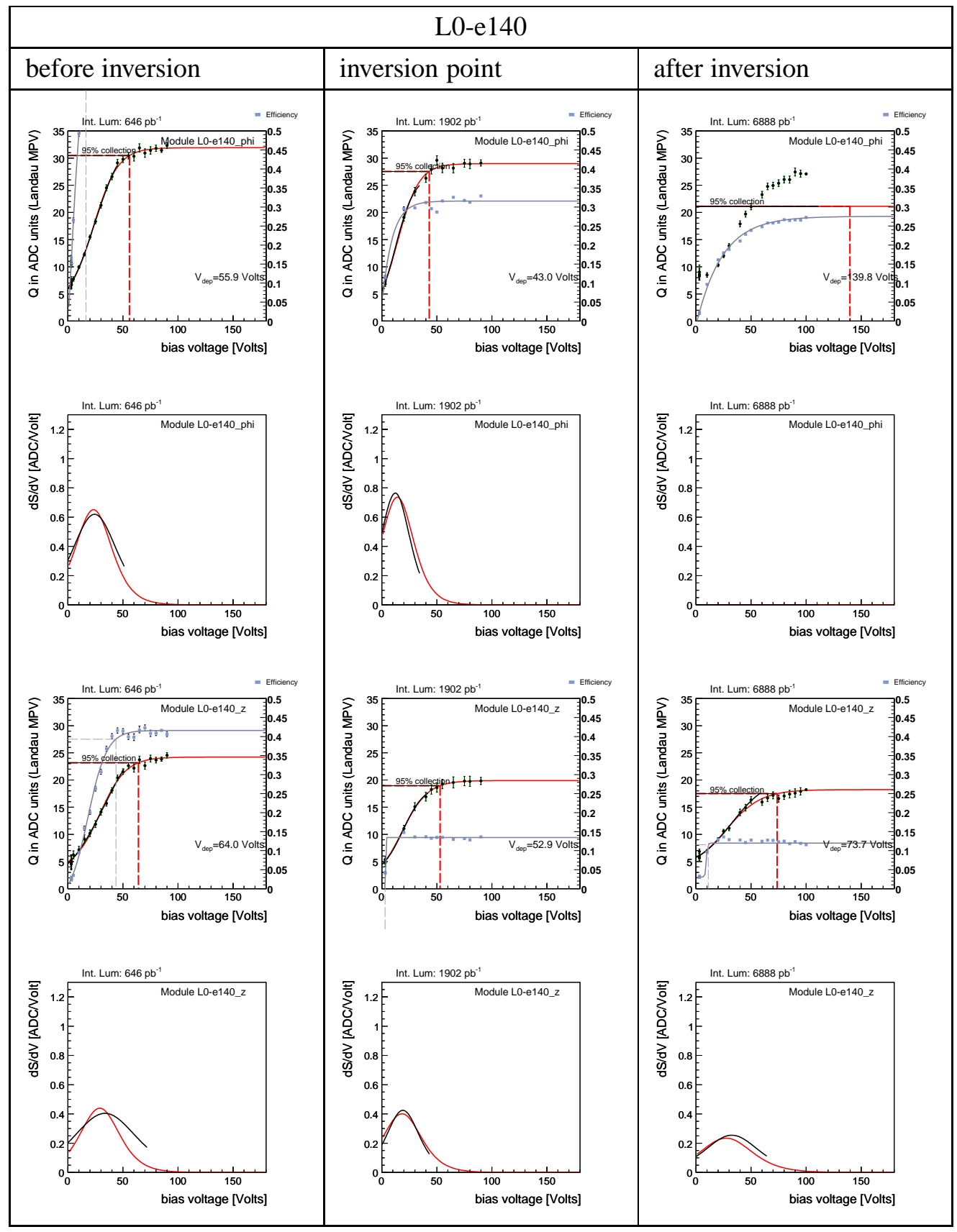


Table 7.12: Type-inversion evolution of cluster size and residuals for L0-e140, a Hamamatsu class sensor. Inversion point at $1902 \mathrm{pb}^{-1}$.

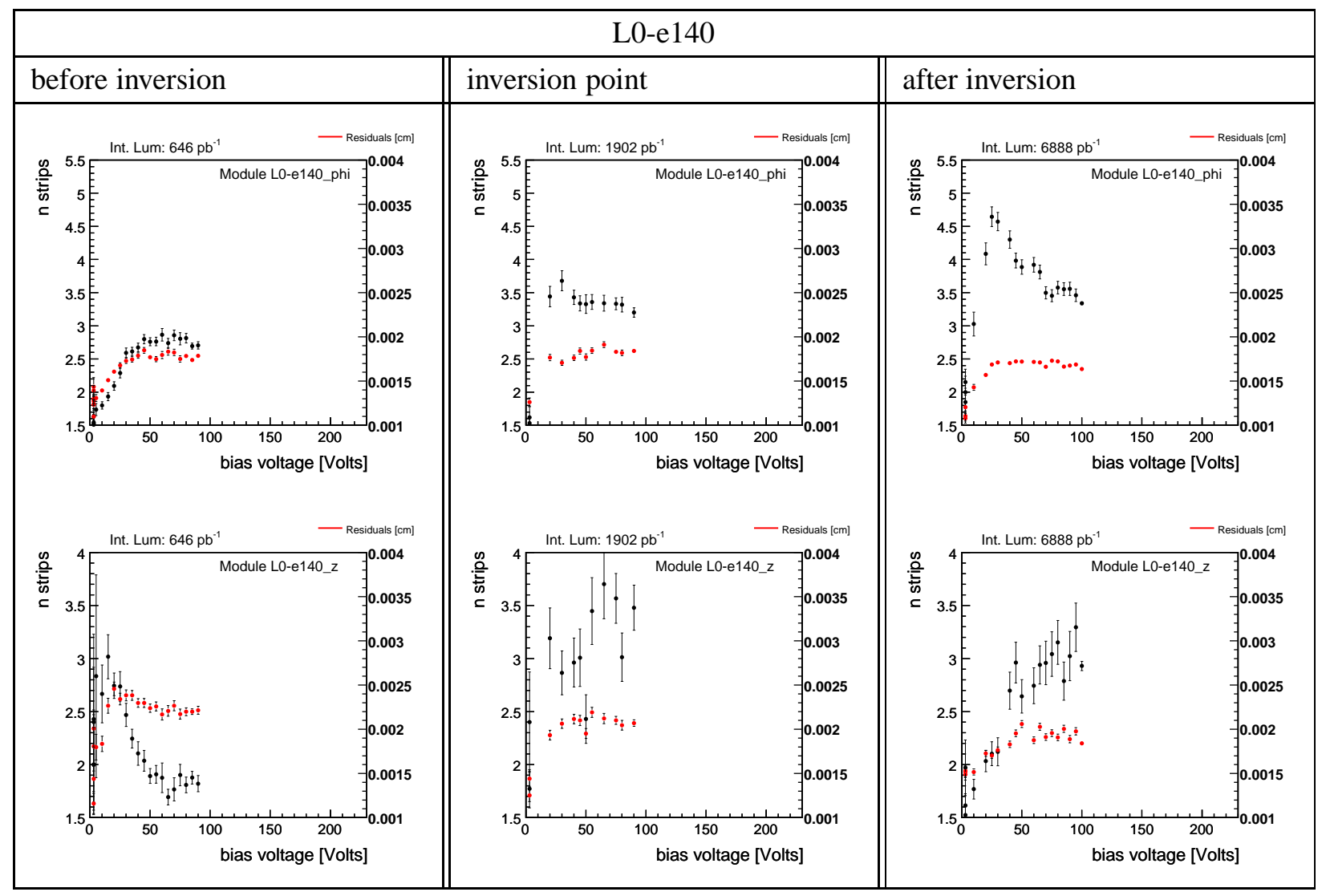


Table 7.13: Type-inversion evolution for L0-e2a0, a Hamamatsu class sensor. Inversion point at $1902 \mathrm{pb}^{-1}$. Each one of the three columns represents a stage point in the lifespan of the sensor ( $p h i$ and $z$ sides are shown): before, after and at inversion. The red sigmoid fits the whole set of charge points while the black sigmoid fits only the subset of points at low voltages. Second row holds the first derivative of those curves, respecting the color code.

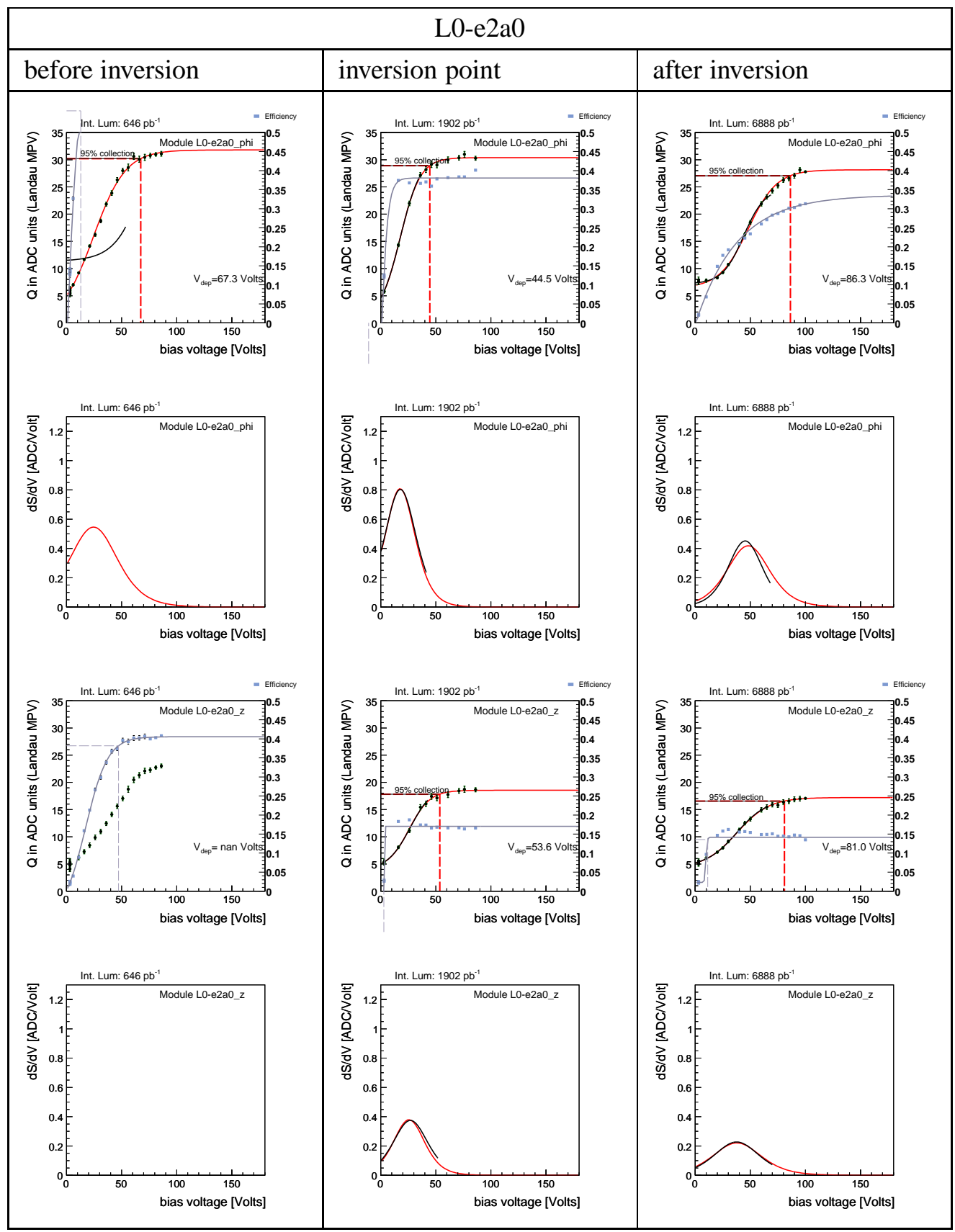


Table 7.14: Type-inversion evolution of cluster size and residuals for L0-e2a0, a Hamamatsu class sensor. Inversion point at $1902 \mathrm{pb}^{-1}$.

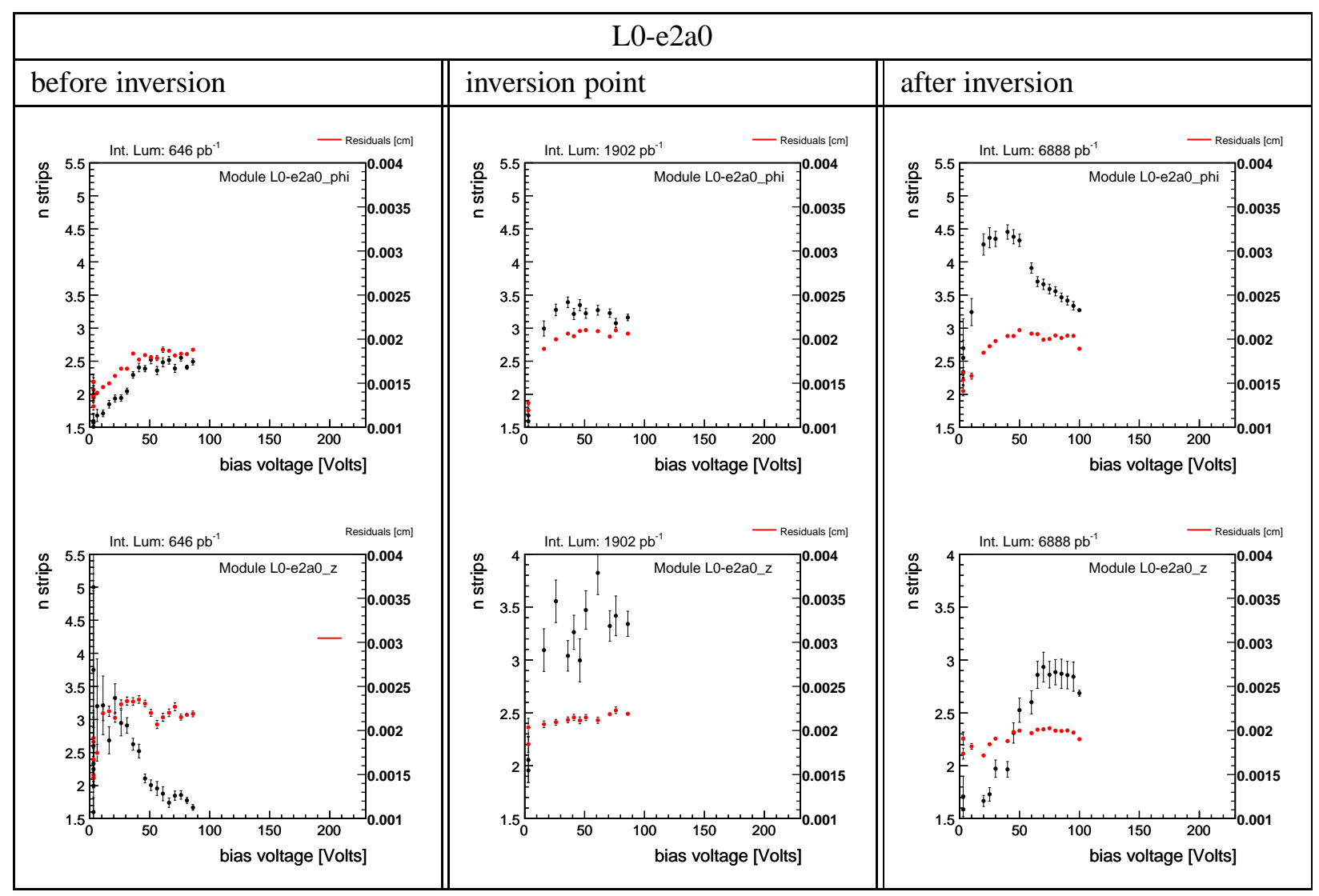


Table 7.15: Type-inversion evolution for L0-e2a0. All luminosities, phi side. Black plot is a fit for low bias voltages (limit of the black fit: $80 \% V_{d} e p$ ).

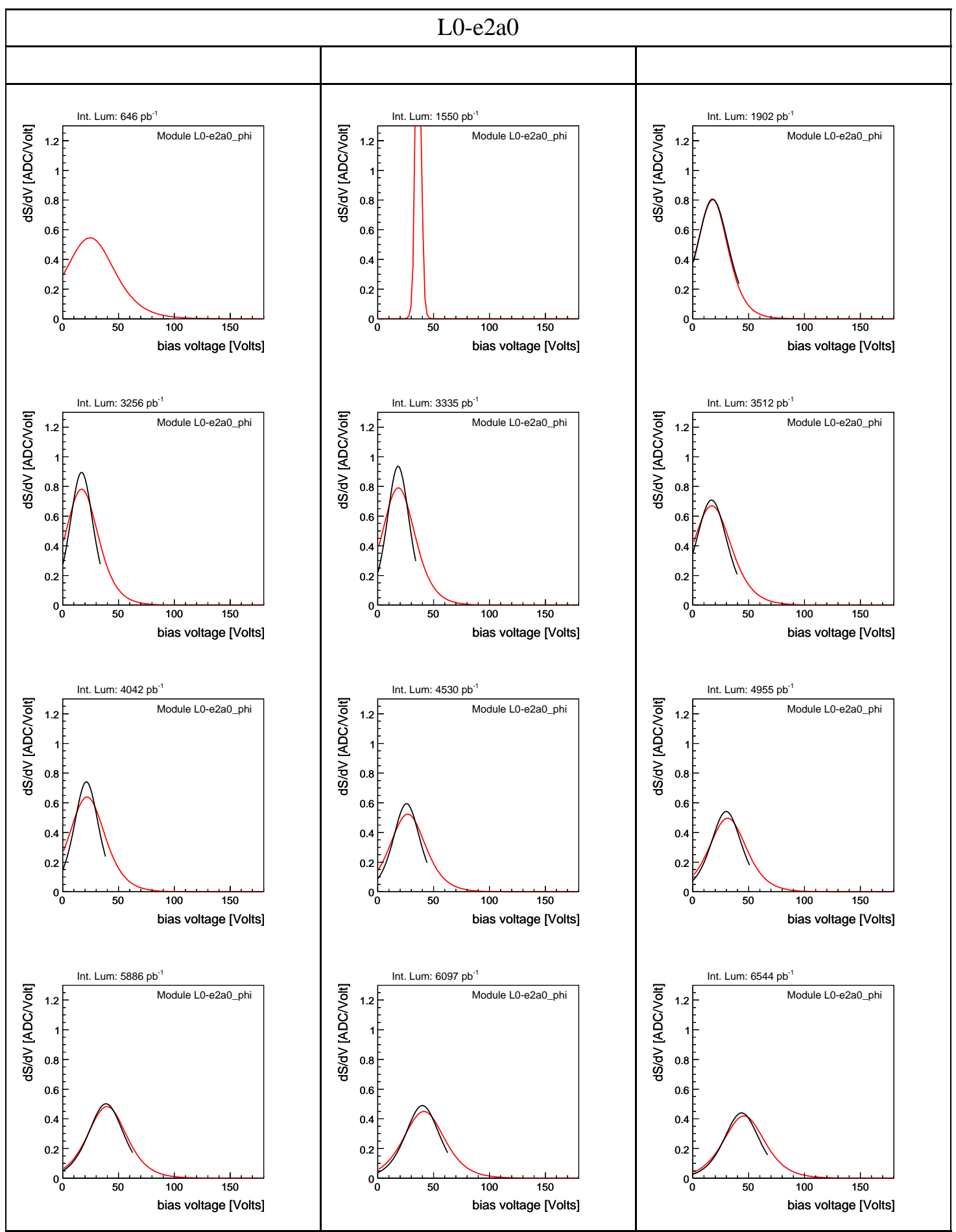


Table 7.16: Type-inversion evolution for L0-e2a0. All luminosities, z side. Black plot is a fit for low bias voltages (limit of the black fit: $80 \% V_{d} e p$ ).

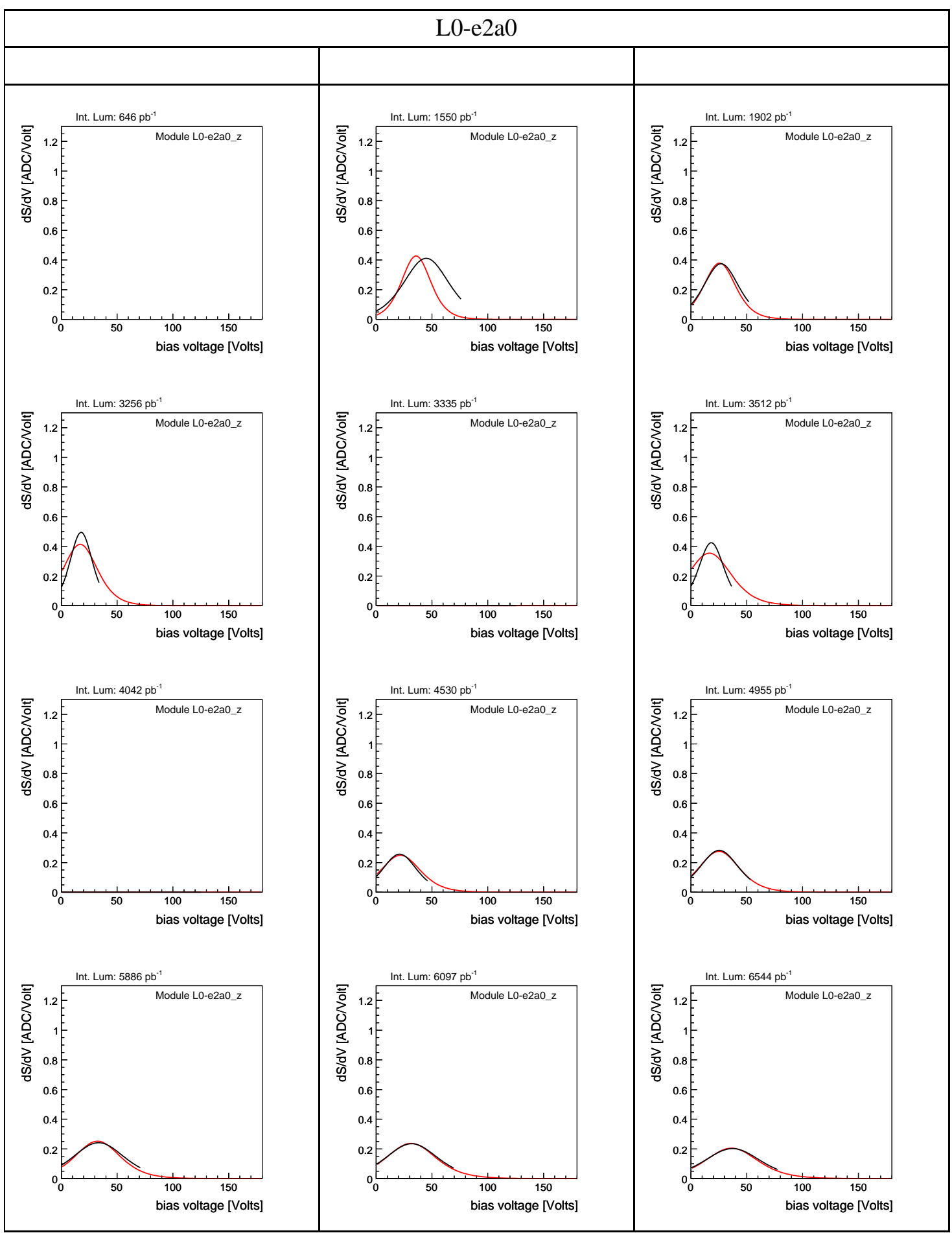




\subsubsection{Summary by layer}

Tables 7.17 and 7.18 are a general view of the behavior at the low-voltage regime of the sensors of LOO and SVX-LO, respectively.

Altough not definitive effects, it is possible however to see two interesting patterns in the plots contained by those Tables. First one, an increase at low luminosities, constant behavior at medium luminosities (around inversion point), and decrease with higher luminosities. This pattern is clearly displayed by L00 sensors of f813 (Micron) and f843 (SGS Thomson), SVX-LO e2a0 $z$ side, and SVX-L0 e090 $\phi$ side. The second possible pattern would be a distribution starting with high points at low luminosities and following with a decrease and eventually constant trend. This behavior is illustrated by sensors L00-f850, SVX-L0 e140 $\phi$ and $z$ sides and SVX-LO e2a0 $\phi$ side.

Table 7.17: Low-voltage behavior of L00 sensors: ordinate at origin of low-voltages sigmoid fit vs luminosities for the three classes of L00 sensors.

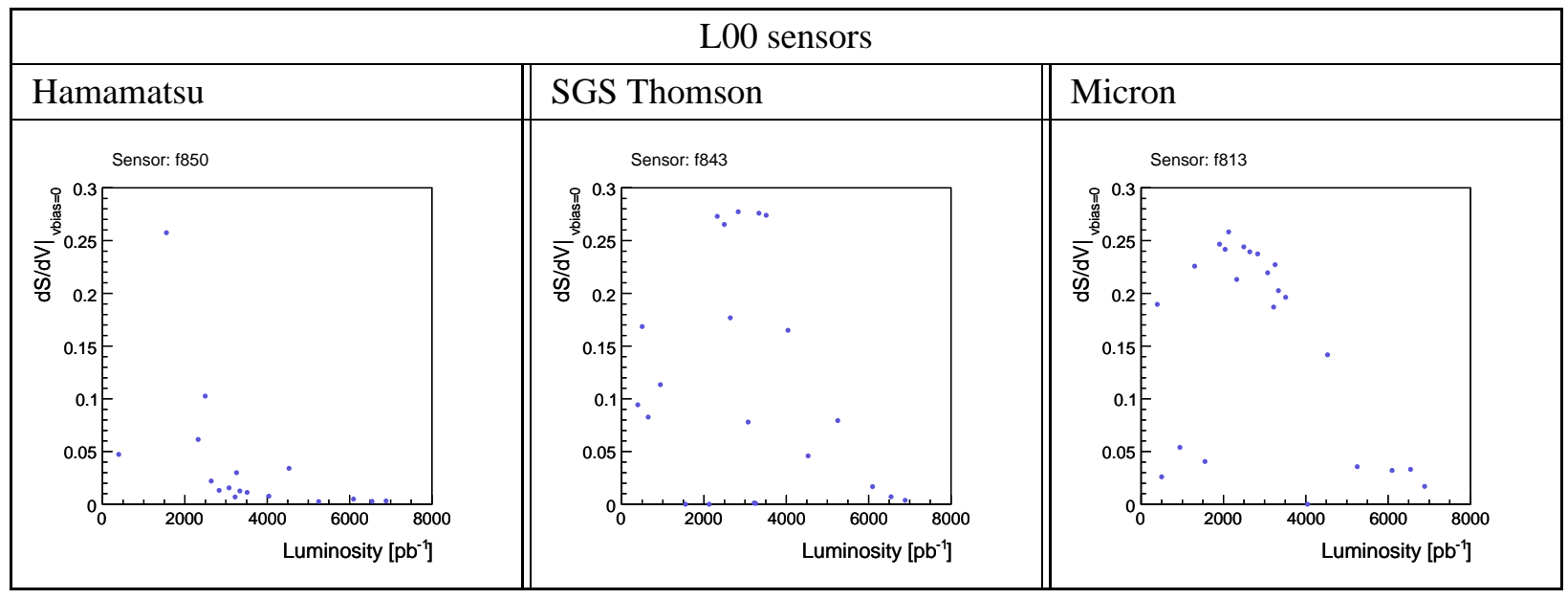


Table 7.18: Low-voltage behavior of SVX-L0 sensors: ordinate at origin of low-voltages sigmoid fit vs luminosities for the three sensors of SVX-L0 with more statistics.

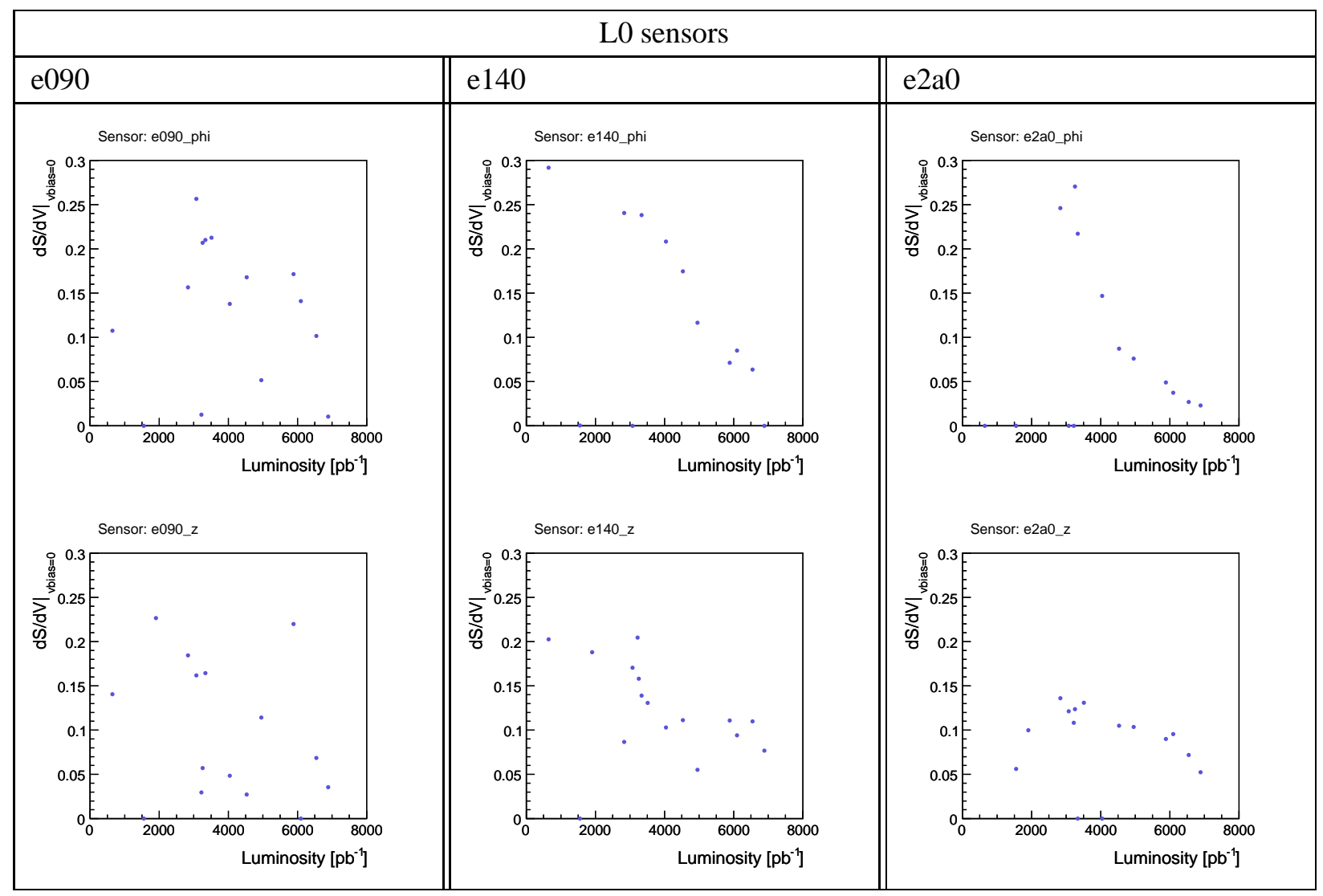




\subsubsection{Behavior at low bias voltage and dopant profile models}

Tables 7.15 and 7.16 and show the behavior at low bias voltage for a set of luminosity points for both sides of sensor e2a0. Similar observations than in the case of L00 can be extracted with the particularity of the double-sided sensor nature of SVX-LO. Let us consider an ideal SVX-LO phi sensor made of an homogeneous initial dopant profile (Fig. 7.8). In such ideal sensor the relationship between net dopant concentration, depletion voltage, depletion space and collectad charge (signal) is described elsewhere (see for example Chapter 1 or Appendix. B). In Figure 7.8, the first column is the situation before irradiation (extrinsic "+" dopant), third column is the inversion point stage (intrinsic "o" dopant) and fifth column is the situation far after inversion (extrinsic "." dopant). Every stage (the three mentioned and the transitions between them represented in colums two and four) is homogeneous, this is, the sum of charged points is the same for each layer.

In the case of a more realistic sensor compatible with observed data (Fig. 7.9) the inversion point would not be intrinsic and stages after inversion would be inhomogeneous, this is, the sum of charged points is differect for each layer and arranged in a way that positive charges are more common in the superior side than in the inferior. To illustrate this effect it has been necessary to draw four charge layers in the stages after inversion.

If we were to start the depletion of this ideal sensor we would see the evolution along luminosity of the signal vs. low bias voltages (to explore the most superficial layers of the sensor) as a linear slope that after some initial irradiation grows faster. When the inversion point is reached, an ideal sensor would display a step function with the step located at $V=0$. In the stages after the inversion point (depletion starting from the $z$ side), the ideal step function would be shifted from the origin in the $x$-axis by a factor equal to the corresponding depletion voltage at each stage. Comparing data of signal vs. bias voltage of SVX-LO sensors (Tables 7.9, 7.11, 7.13) with this ideal model we can check that stages before irradiation behave as expected but the inversion point stage and subsequents do not. Data of the inversion point stage displays an important mismatch with the inversion modelization (for example, Fig. 6.26). In the model, the depletion voltage drops to zero while data display a minimum located at some tens of volts above zero, depending on the class of the sensor. Data from stages after irradiation (plots in the third column of Tables 7.9, 7.11, 7.13) show that what should be an ideal step function is closer to a sigmoid with slope decreasing along luminosity.

The ideal case for a SVX-LO $z$ sensor would be the reverse situation along luminosity than for the phi side (Fig. 7.8). In the $z$ case, divergences between data and the corresponding ideal stages of the function of signal vs. low bias voltage start from the beginning of the irradiation. 

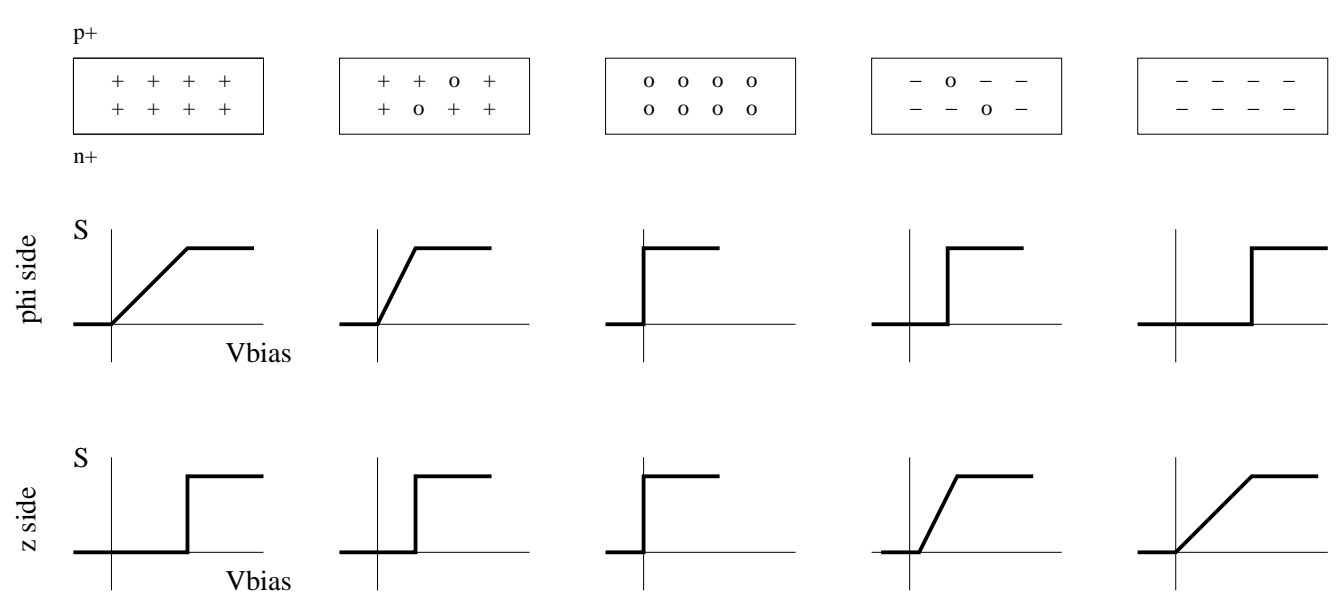

Figure 7.8: Ideal SVX-L0 sensor space charge (homogeneous dopant profile) and signal vs. bias voltage curves behavior along luminosity.
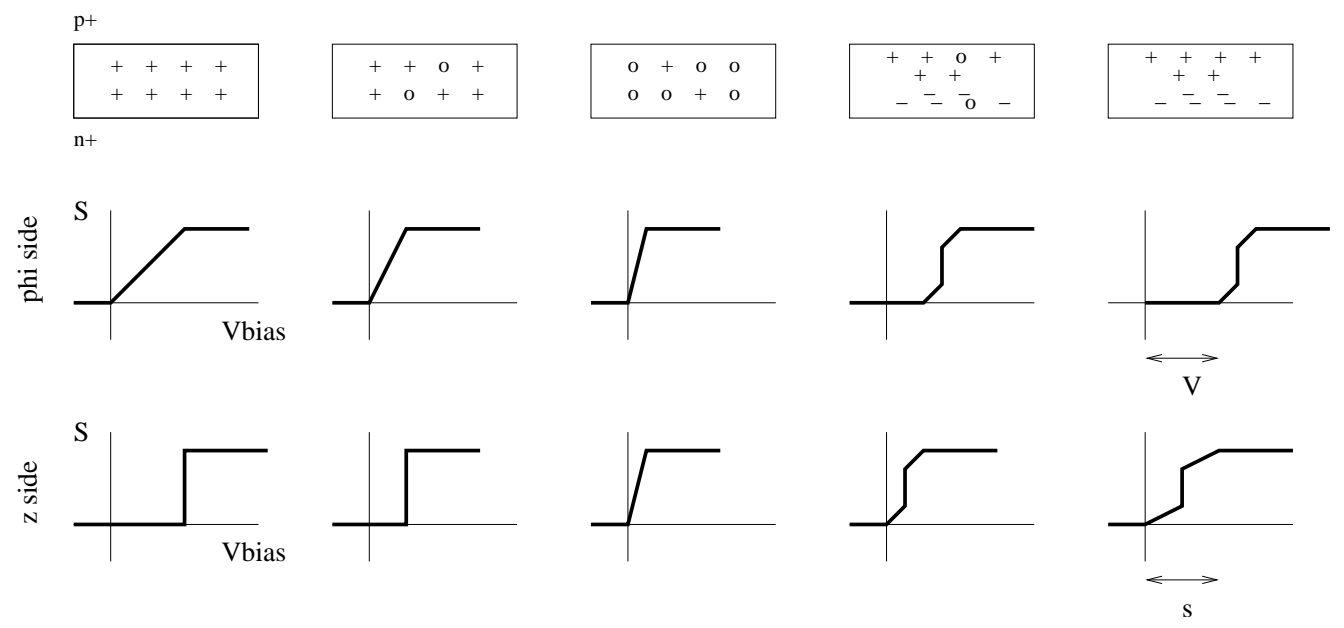

Figure 7.9: Real SVX-L0 sensor space charge (inhomogeneous dopant profile, double junction like) and signal vs. bias voltage curves behavior along luminosity. The presence of a slope $s$ in the experimental curves could be an evidence of double-junction dopant profile structure (Appendix D). 


\section{Chapter 8}

\section{Conclusions}

This work has been devoted to the study of the radiation damage effects observed on the CDF silicon detectors, in particular the dopant type-inversion and the evolution of the bias currents.

The CDF silicon detectors were designed to be replaced after 2-3 $\mathrm{fb}^{-1}$ of recorded data. However, the planned replacement was not possible and the sensors have been operated until 10-12 $\mathrm{fb}^{-1}$. This particular circumstance has been a challenge for the operation and maintenance of the silicon detectors and the reason behind a meticulous monitoring work.

In this document the radiation damage has been studied from two viewpoints: the bias current and the bias voltage. Other radiation damage effects and aging over different detector parameters (efficiency, cluster size...) have also been observed and recorded.

The study of the bias currents is of relevance in an irradiated silicon sensor since it is a parameter linked to the received fluence. Hence and by studying data from bias current it is possible to relate the luminosity, a known quantity, with the total dose received by the detector. The results expressed as a function of the fluence become independent of the local environment and can be useful for other experiments.

Regarding the bias voltage, its evolution with time shows the type-inversion for L00 and layer SVX-LO. Since the silicon detectors are the limitant factor in the lifespan of the whole CDF experiment, extrapolations of this plots of inversion have been the reference for the periodical inspections of the commissioners of the U.S. Department Of Energy and the annual budget reports.

The scientific importance of the dopant type-inversion becomes evident since current experimental data do not perfectly match the Hamburg model. In the model, the minimum of the inversion curve drops to zero volts while the inversion curve of the CDF data displays a shift in voltage, being located between 30 and 45 volts. Since an account of the internal 
resistance of the bias circuit would only contribute with about 10 volts, the remaining voltage difference can not be explained with the current model. The type-inversion has also been illustrated -with less accuracy tough- from the viewpoint of the dopant profile at low bias voltages. Altough some radiation-generated complexes inside the sensor's volume are well known and able to explain the macroscopic results, the deeper mechanisms behind typeinversion are many and still unknown. Dopant type-inversion is still an open question in the areas of material science and solid state physics.

The results collected in this work have been shown to the public in several talks and meetings between 2008 and 2009:

* "Longevity and Radiation Aging Studies of the CDF II Silicon Detectors", Talk at the American Physical Society Meeting, Saint Louis MO, April 2008.

* "Longevity Studies of the CDF II Silicon Detectors", Talk at the CMS Symposium, Fermilab, November 2008.

* "Longevity Studies of the CDF II Silicon Detectors", Talk at the CIEMAT High Energy Physics Christmas Workshop, Madrid, December 2008.

* "Longevity Studies of the CDF II Silicon Detectors", Talk at the All Experimenters Meeting, Fermilab, January 2009.

* "The CDF RUN II Silicon Detectors: Longevity Studies", Poster at the 11th Pisa Meeting on Advanced Detectors, Elba, May 2009.

A detailed description of the behavior of the CDF silicon detectors is being written for Nuclear Instruments and Methods A with the title "Operational Experience, Improvements, and Performance of the CDF Run II Silicon Vertex Detector”. 


\section{Appendix A}

\section{Resumen en Castellano}

\section{A.1 El experimento CDF en el Laboratorio Federal Fermi}

EI Laboratorio Federal Fermi es un centro internacional de investigación en Física de Altas Energías localizado en las proximidades de Chicago, Ilinois (EE.UU.). Sus instalaciones albergan al Tevatrón, el mayor acelerador de partículas actualmente opertativo en el mundo. El Tevatrón acelera protones y antiprotones hasta una energía en el centro de masas de $2 \mathrm{TeV}$ y los focaliza en dos puntos distintos del anillo para provocar su colisión. En esos puntos están situados los detectores DO y CDF. Estos aparatos tienen unas dimensiones aproximadas de 15 metros cúbicos y 5 toneladas de peso (Figura 1).

El departamento de Investigación Básica del CIEMAT se incorporó al experimento CDF en 2005 y su interés se centra en la recogida y análisis de datos de la última fase de este proyecto (Run II). Como parte de las responsabilididades de la colaboración, el CIEMAT debe encargarse concretamente del mantenimiento del detector de vértices de silicio. Este dispositivo está sellado en la parte más interna de CDF y en contacto con el conducto del haz (beampipe). Por este motivo es además la parte que más radiación recibe procedente del punto de colisión. El detector de silicio es una herramienta fundamental para el análisis de los procesos subnucleares.

\section{A.2 Materiales de estado sólido para la detección de radiación ion- izante.}

Las partículas cargadas depositan por ionización una fracción de su energía al atravesar cualquier material aunque sólo algunos de ellos sean adecuados como dispositivos detec- 


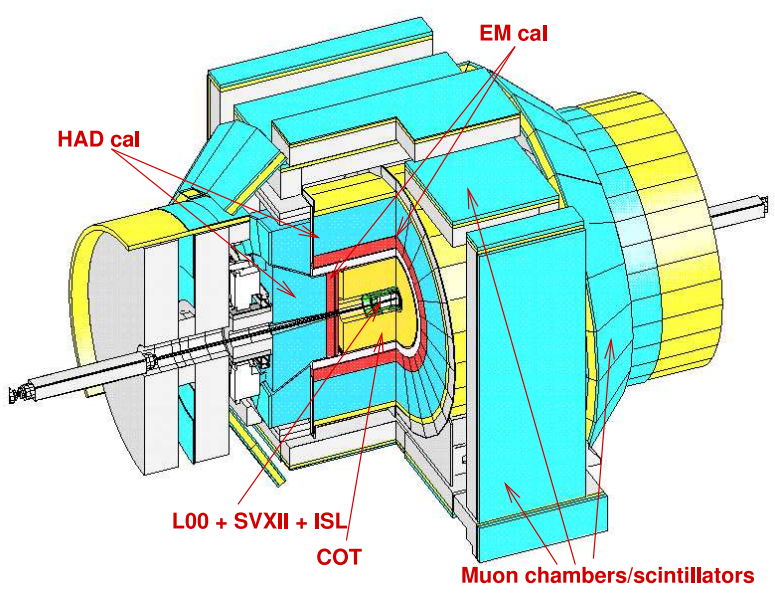

Figure A.1: Detector CDF. La dirección de avance de los protones determina el sentido positivo de la coordenada cartesiana $z$. El detector más interno es el de silicio (L00+SVXII+ISL) y hacia el exterior se sitúan la cámara central de trazas (Central Outer Tracker,COT), los calorímetros electromagnético y hadrónico (EM cal, HAD cal) y las cámaras de muones.

tores. El modelo de detector conceptualmente más simple consistiría en un material aislante o semiconductor entre dos placas de metal (condensador) sobre las que se aplica el voltaje (tensión de polarización) para crear un campo eléctrico en el interior del medio detector pasivo de forma que se pueda recoger la señal en los electrodos. Este elemento detector pasivo puede ser un gas (e.g. cámara de ionización), un líquido (e.g. calorímetro de argón líquido) o un sólido (e.g. detector de diamante).

EI silicio ha encontrado nuevas y revolucionarias aplicaciones desde mediados del siglo XX, siendo usado en diferentes áreas como la electrónica, las comunicaciones, los ordenadores, las células fotovoltaicas, la espectrometría gamma y de rayos $\mathrm{X}$, biosensores, mecanismos micro electromecánicos (MEMs) y detectores de partículas. Estos últimos utilizan como material detector un cristal de silicio en el cual la radiación incidente transmite parte de su energía a un cierto número de electrones que promocionarán desde la banda de valencia a la de conducción, generándose así pares electrón-hueco. Bajo la influencia del campo eléctrico externo, tanto los electrones como los huecos viajarán hacia los electrodos donde darán lugar a un pulso eléctrico. Este proceso de ionización del semiconductor es equivalente al fenómeno de ionización que sucedía en las cámaras de niebla. Comparados con éstas, la densidad de un dispositivo semiconductor es mucho mayor y las partículas cargadas de alta energía pueden registrarse mediante detectores relativamente pequeños. 


\section{A.2.1 Detectores de vértices de silicio: el detector SVX de microbandas de CDF.}

Los detectores de microbandas de silicio son esencialmente una disposición de diodos semiconductores (unión $p n$ en polarización inversa y acoplo capacitivo) independientes, ubicados sobre un sustrato común tipo $\boldsymbol{n}$ de alta resistividad. La matriz cristalina de este sustrato es de clase cristalográfica $<\mathbf{1 1 1}>$ o bien de tipo $<\mathbf{1 0 0}>$ ya que proporcionan la máxima densidad y por tanto se reduce el riesgo de que el aluminio de la armadura colectora migre hacia el interior del silicio en la fase de fabricación, lo que provocaría el cortocircuito de la estructura del diodo. Sobre este monolito de silicio se procede al dopaje de tipo $p$ a lo largo de bandas, creándose de esta manera las uniones $\mathrm{pn}$. Se metaliza posteriormente con aluminio que servirá como electrodo y el diodo resultante es polarizado inversamente. Para producir dichas estructuras colectoras (implante, electrodo) se utilizan tecnologías estándar de la industria microelectrónica que permiten crear altas densidades de instrumentación (una banda cada 10-100 $\mu \mathrm{m}$ ) de forma fiable y económica. A esta unidad detectora la llamaremos celda. Si se ordenan miles de estas celdas de manera concéntrica y bajo simetría cilíndrica en torno al punto de colisión, queda reticulado el espacio donde tienen lugar los choques entre partículas (vértice principal) y donde van a desintegrarse las partículas de los estados intermedios (vértices secundarios). Puesto que este retículo sensor proporciona los puntos por donde han pasado las partículas, podremos reconstruir por extrapolación a partir de esos puntos sus trayectorias (trazas) y vértices de desintegración, fundamentales para comprender la física subyacente. Los detectores de silicio tienen una resolución para la reconstrucción de trazas del orden de las $\mathbf{1 0}$ micras, mayor que las cámaras de niebla o las cámaras de hilos. Como desventaja, el silicio resulta más caro y necesita una cuidadosa refrigeración (del orden de $\mathbf{- 1 0}{ }^{\circ} \mathrm{C}$ ) para evitar la presencia de corrientes de fuga (ruido) que deteriorarían la calidad de los datos. Una refrigeración inadecuada acelera además el daño por radiación.

El detector de silicio de CDF consta de tres subsistemas: L00, SVX e ISL (Figura A.2). L00 es el dispositivo más interno y permite mejorar la resolución del parámetro de impacto. SVX cuenta con cinco capas concéntricas de silicio y es el elemento fundamental para la reconstrucción de trazas y vértices. ISL está compuesto por dos capas intermedias de silicio entre SVX y la cámara de trazas COT (Figura 3), que proporciona datos suplementarios para la reconstrucción de las trazas. Cada cristal de silicio tiene unas dimensiones del orden de decenas de $\mathrm{cm}^{2}$ y un grosor de 300 micras. Para detectores más delgados no sería aceptable la razón de señal a ruido para este tipo de experimentos de reconstrucción de trazas. El sistema cuenta con 700 celdas sensoras (772.000 canales de lectura) que suman $6 \mathrm{~m}^{2}$ de silicio. 


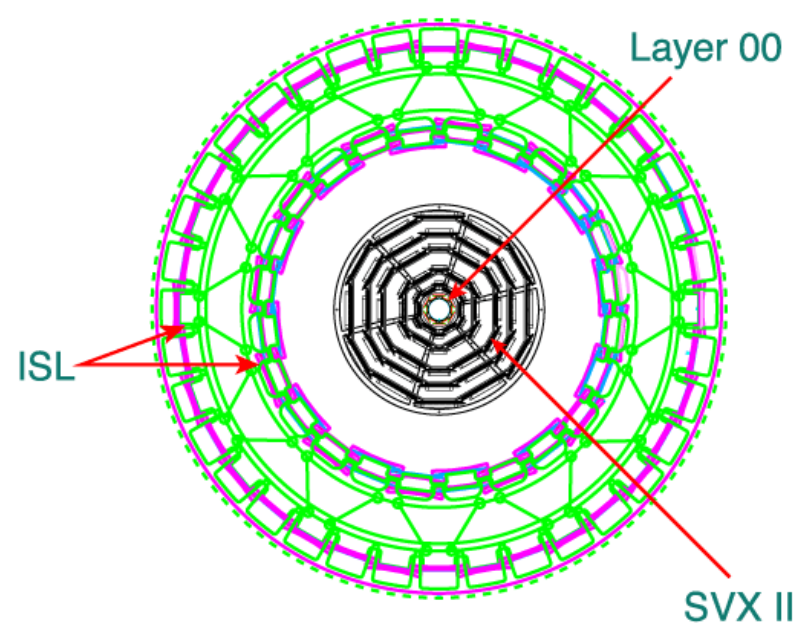

$64 \mathrm{~cm}$

Figure A.2: Sección transversal del detector de silicio. Se muestran los tres subsistemas que lo integran: L00, SVX-II e ISL.

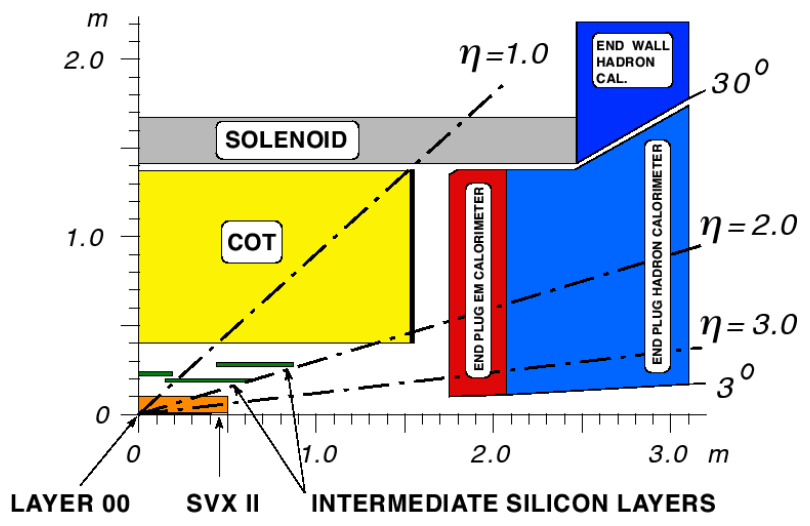

Figure A.3: Sección longitudinal de CDF y rangos de detección para los distintos subdetectores. Se muestra el primer cuadrante del plano cartesiano $Y Z$. La coordenada $\eta$ (pseudo-rapidez) es una transformación del ángulo polar $\theta$ según la fórmula $\eta=-\operatorname{Ln}(\operatorname{tg}(\theta / 2))$. 


\section{A.2.2 Daños por radiación.}

El efecto del daño por radiación en dispositivos de silicio ha sido estudiado durante décadas y de manera intensiva pero el problema es todavía vigente dado el gran número de nuevos artefactos sometidos a dosis hostiles de radiación y que no pueden recibir mantenimiento, como ocurre en sondas espaciales, satélites, reactores de fusión y detectores internos (sellados durante años) en aceleradores de partículas. En todos estos casos no existen estudios adecuados para la predicción de la vida útil bajo esas condiciones de trabajo.

Consideremos una radiación electromagnética de energía correspondiente al espectro visible. Sus efectos sobre los dispositivos de silicio cristalino (creación de pares electrónhueco) son completamente transitorios: los pares generados se recombinan rápidamente. Pero si se incrementa la energía de la radiación, los portadores más energéticos aumentan su probabilidad de alcanzar la capa de óxido, provocando cierto grado de daño superficial. Si la radiación incidente excediese los $250 \mathrm{keV}$, la energía sería suficente como para desplazar átomos de silicio de sus posiciones en la red. A este caso se le conoce como daño por desplazamiento. Para el caso de radiación por partículas cargadas y con masa el daño por desplazamiento sucede para una energía mucho menor. Los protones de baja energía son extremadamente peligrosos debido a la gran sección eficaz para la dispersión de Coulomb en silicio de tipo $p$. Estos dos mecanismos constituyen la base de todos los efectos por radiación que nos conciernen en el caso de los detectores de silicio y la electrónica asociada. Comprender estos efectos resulta de vital importanca para la correcta interpretación de los datos.

\section{A.3 Aplicaciones a la Física de Altas Energías.}

\section{A.3.1 Importancia de los detectores de vértices de silicio.}

Se ha expuesto anteriormente cómo un detector de silicio es capaz de proporcionar trazas con gran precisión. Dos trazas próximas reconstruidas con la suficiente resolución pueden extrapolarse hasta el vértice original. Este vértice representaría la posición en la que la partícula madre se desintegró en las partículas hijas. En este caso hablaríamos de espectroscopía sin desplazamiento. Si una de las partículas hijas diera lugar también a un nuevo vértice de desintegración quedaría definida $L_{x y}$ entre ambos vértices, dando lugar al caso de espectroscopía con desplazamiento. En esta situción, además, podríamos utilizar la distancia entre vértices $L_{x y}$ para reconocer (tagging) un determinado tipo de quark, siendo así posible la búsqueda de nuevas partículas. 


\section{A.3.2 Espectroscopía de hadrones.}

La teoría de la interacción fuerte, la Cromodinámica Cuántica (QCD), está construída sobre partículas todavía más fundamentales: los quark y los gluones. En teoría sería posible calcular las propiedades ya observadas de los hadrones a partir de las de los gluones y quarks. En la práctica, esto es muy difícil de conseguir. La brecha entre la teoría de campo subyacente y los observables hadrónicos implica la resolución del problema general de una teoría de campos cuánticos. El único intento directo contra este problema consiste en buscar una solución numérica para las ecuaciones de campo en una red de espacio-tiempo discretizada, técnica conocida como Laticce $Q C D$. También puede procederse de manera indirecta, donde se utilizan modelos simplificados de la propia QCD. De esta manera se ha trabajado, con éxito, en otras ramas de la física como Materia Condensada o Física Nuclear. En el primer caso, por ejemplo, la teoría completa de la Electrodinámica Cuántica (QED) se sustituye por una teoría efectiva de electrones no relativistas, huecos, pares de Cooper, potenciales de ligadura, etcétera. En el estudio del núcleo atómico, la interacción fuerte es equivalente, a su vez, a una fuerza efectiva entre sólo dos nucleones.

Similarmente para la escala hadrónica y utilizando modelos aproximados consistentes con los datos experimentales, se puede avanzar en la comprensión del número de grados de libertad efectivos que caracterizan las interacciones en el interior del hadrón. La espectroscopía de hadrones permite conocer las magnitudes que caracterizan a una partícula: la masa, el espín, el momento angular, la paridad, la energía y la vida media. Los tiempos de vida de partículas tales como piones o kaones les permiten recorrer distancias del orden de centímetros. Trazas de este tipo pueden reconstruirse, por tanto, mediante un instrumento no demasiado preciso. Pero las energías de colisión actuales producen partículas con un tiempo de desintegración mucho más breve ( $L_{x y}$ del orden de micras) cuyos productos de desintegración sólo pueden reconstruirse con suficiente precisión mediante un detector de silicio.

Únicamente con estos detectores se tiene información lo suficientemente precisa como para reconstruir el punto donde la partícula de vida corta (normalmente conteniendo un quark pesado) se desintegra, y reconstruir a partir de sus productos de desintegración sus propiedades características.

Utilizando esta técnica se ha descubierto, por ejemplo, el fenómeno de mixing u oscilación materia-antimateria en mesones $B_{S}$ y el tiempos de desintegración de partículas que contienen al quark $b$. 


\section{A.3.3 Búsquedas.}

De manera análoga, la búsqueda de nuevas partículas e interacciones hace uso del detector de silicio para la clasificación (tagging) de chorros de partículas que contengan quarks pesados como el $c$ o el $b$. Esa clasificación consiste en primer lugar, en la búsqueda de vértices secundarios de desintegración en la zona del espacio correspondiente al paso de jets (depósitos de energía bien localizados en el calorímetro). La reconstrucción precisa de vértices secundarios y su desplazamiento requiere el detector de silicio debido a las distancias implicadas, como se vio en el apartado anterior. Posteriormente esa distancia entre vértices se compara con la longitud de desintegración de los quarks $c$ o $b$. En particular para el caso del $b$ esa distancia es aproximadamente de 200 micras y por tanto, el chorro de partículas que contenía el vértice desplazado puede clasificarse como de tipo $b$, lo que simplifica considerablemente el análisis. El quark top se desintegra en un bosón $W$ y en un quark $b$ y pudo descubrirse (1996) mediante la selección de sucesos que contenían al quark $b$. Este mismo procedimiento es también adecuado para la búsqueda de nuevas partículas como el bosón de Higgs cuya desintegración produce un estado final conteniendo quark $b$ y antiquark $\bar{b}$.

Aunque ningún experimento ha podido detectar la existencia del bosón de Higgs, hay evidencias indirectas para creer en ella. El bosón de Higgs se predijo en 1964 como el cuanto de un cierto campo escalar. El valor medio de este campo en el vacío (VMV) es constante e igual a $246 \mathrm{GeV}$. La existencia de este campo es fundamental para ciertos procesos físicos ya que dotaría de masa al resto de partículas elementales incluyendo la del propio bosón de Higgs. En particular, la adquisición de un VMV distinto de cero rompe espontáneamente la simetría gauge electrodébil. A este fenómeno se le conoce como Mecanismo de Higgs y es la única forma conocida capaz de explicar el origen de la masa de los bosones de gauge, siendo además compatible con las propias teorías gauge. Para la masa de la partícula de Higgs existe una cota inferior experimental de 114'5 GeV proporcionada por el experimento LEP e indicios para el límite superior en torno a $200 \mathrm{GeV}$. Este rango de energías puede explorarse mediante los colisionadores de hadrones de Fermilab y de CERN y confirmarían completamente la teoría del Modelo Estándar.

Más allá de este modelo y en el contexto de las Teorías de Gran Unificación se encuentra la hipótesis de la Supersimetría en la que para cada partícula fermiónica de la naturaleza existe asociada una partícula compañera bosónica y viceversa. Según esta hipótesis, el quark $\tilde{b}$ (sbottom), compañero supersimétrico del quark $b$, se desintegraría en un quark $b$ más un neutralino $\left(\tilde{\chi}^{\circ}\right)$, compañero supersimétrico del neutrino y partícula relacionada con el problema de la materia oscura. El quark sbottom podría ser encontrado en Tevatrón utilizando 


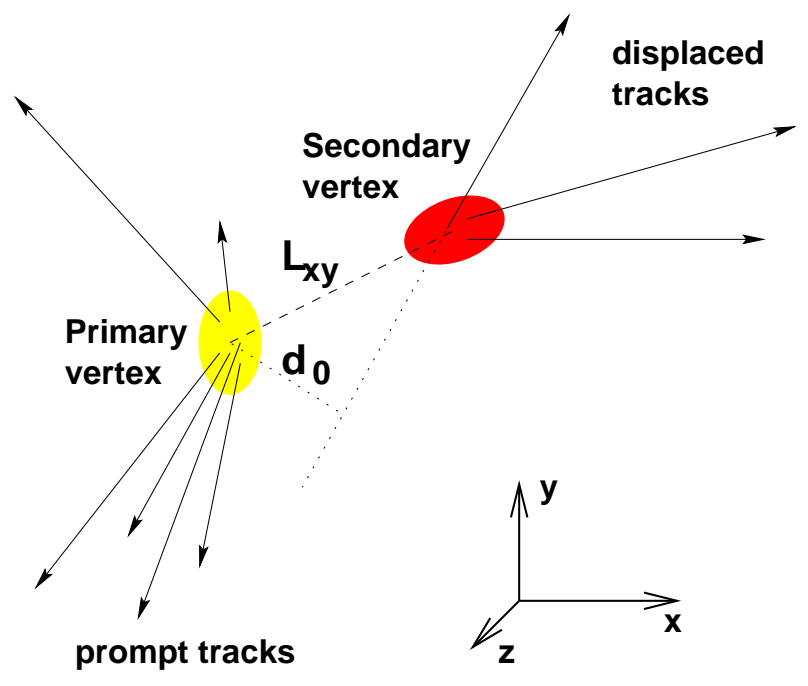

Figure A.4: Vértices de colisión. $L_{x y} \sim 200 \mu \mathrm{m}$

la clasificación de jets de tipo $b$. En consecuencia, el método de clasificación de chorros de partículas mediante la medida de la distancia entre vértices de reconstrucción serviría así para avanzar en esta nueva frontera de la ciencia.

\section{A.4 Exploraciones de voltaje de polarización. Exploración de señal vs. polarización.}

En CDF periódicamente se llevan a cabo ciertas pruebas para evaluar el voltaje de desertización de los sensores. Existen dos maneras de aproximarse a este propósito: exploración de ruido vs. polarización y exploración de señal vs. polarización. La exploración de ruido vs. polarización es válido para sensores de doble cara y por tanto no puede practicarse para L00. En el caso de la exploración de señal vs. polarización, la carga depositada en el sensor por una partícula incidente se mide como función del voltaje de polarización al que el sensor está sometido. Para cada valor de voltaje de polarización, la distribución de carga se ajusta a una curva de landau convolucionada con una curva gaussiana. El Valor Más Probable (VMP) de esa función convolucionada se define como la carga recolectada por el sensor estando polarizado a ese voltaje. Por tanto, para todo el conjunto de puntos de polarización se consigue un correspondiente conjunto de puntos de carga. A continuación se ajusta este nuevo conjunto mediante una curva sigmoide y el voltaje de desertización del sensor se define como el valor de la altura de la curva al $95 \%$ de su valor de saturación (Fig. A.5). Este método funciona para todos los sensores de CDF: L00, SVX-II y ISL. Sin embargo la desventaja del mismo es que la recolección de carga debe hacerse con colisiones 
reales para lo que debe utilizarse tiempo útil del Tevatron.
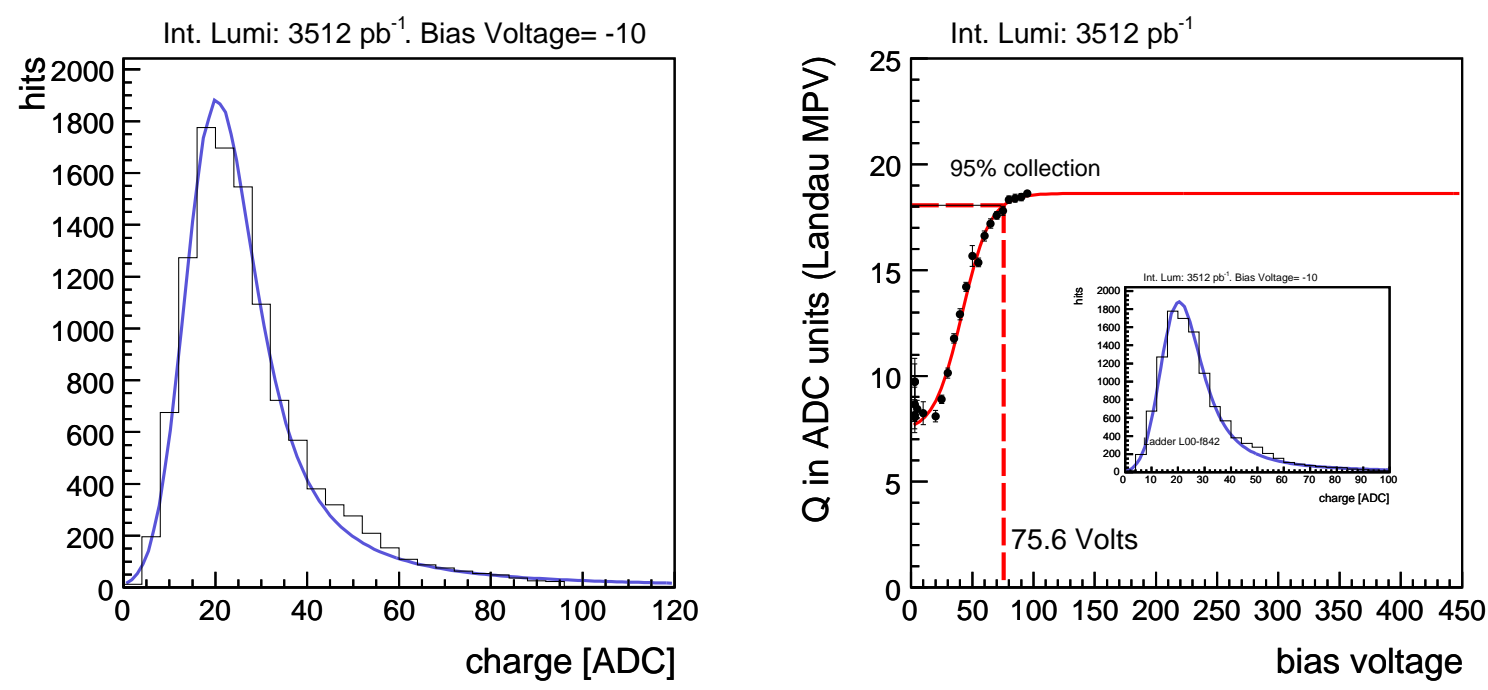

Figure A.5: Ejemplo del método de exploración de señal vs. polarización. Esta prueba se practicó para L00 a una luminosidad integrada de $3512 \mathrm{pb}^{-1}$. La figura de la izquierda muestra la distribución de la colección de carga (a un voltaje de polarización de 10 voltios por debajo del voltaje nominal de operación del sensor) correspondiente a uno de los 48 modulos de L00 y su curva de ajuste (landau) caracterizada por su valor más probable (VMP). La figura de la derecha muestra el conjunto de 25 VMPs correspondiente a los 25 voltajes de polarización. Este conjunto de puntos se ajusta mediante una curva sigmoide cuyo 95\% del valor de su altura, 75.6 voltios, se considera como el voltaje de desertización de este particular sensor y para la luminosidad de $3512 \mathrm{pb}^{-1}$. Este valor del voltaje de desertización es el resultado del método de exploración de señal vs. polarización.

\section{A.4.1 Resultados de las exploraciones de señal vs. polarización para L00.}

\section{A.4.1.1 Voltaje de desertización}

Los Valores Más Probables (VMP) de las curvas de ajuste se muestran en las figuras A.6, A.7, A.8, y A.9 para cuatro sensores de ejemplo de LOO. 

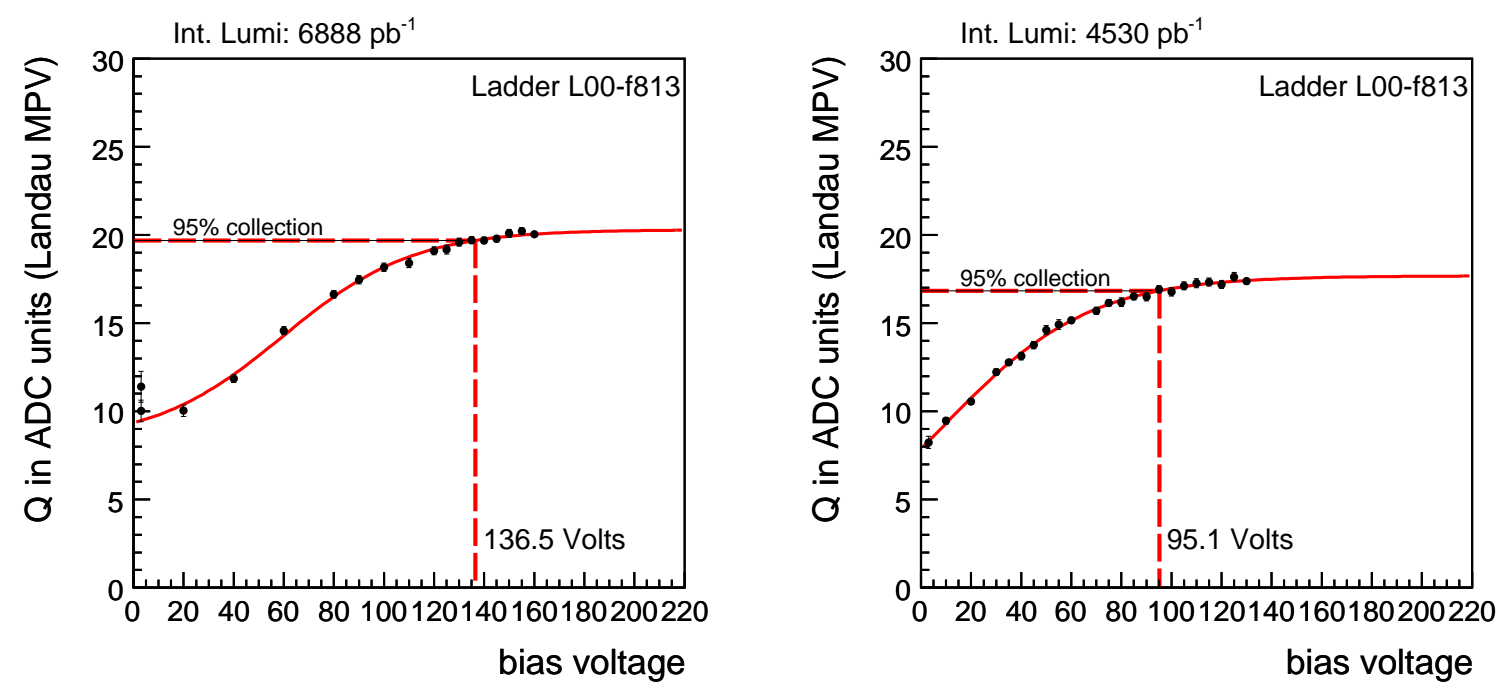

Figure A.6: Figuras de voltaje de desertización para el sensor f813 de clase Micron de L00. Se observan pequeños incrementos en el voltaje de desertización para este sensor desde la última prueba a $4530 \mathrm{pb}^{-1}$, un resultado esperable dado el especial diseño de estos sensores.
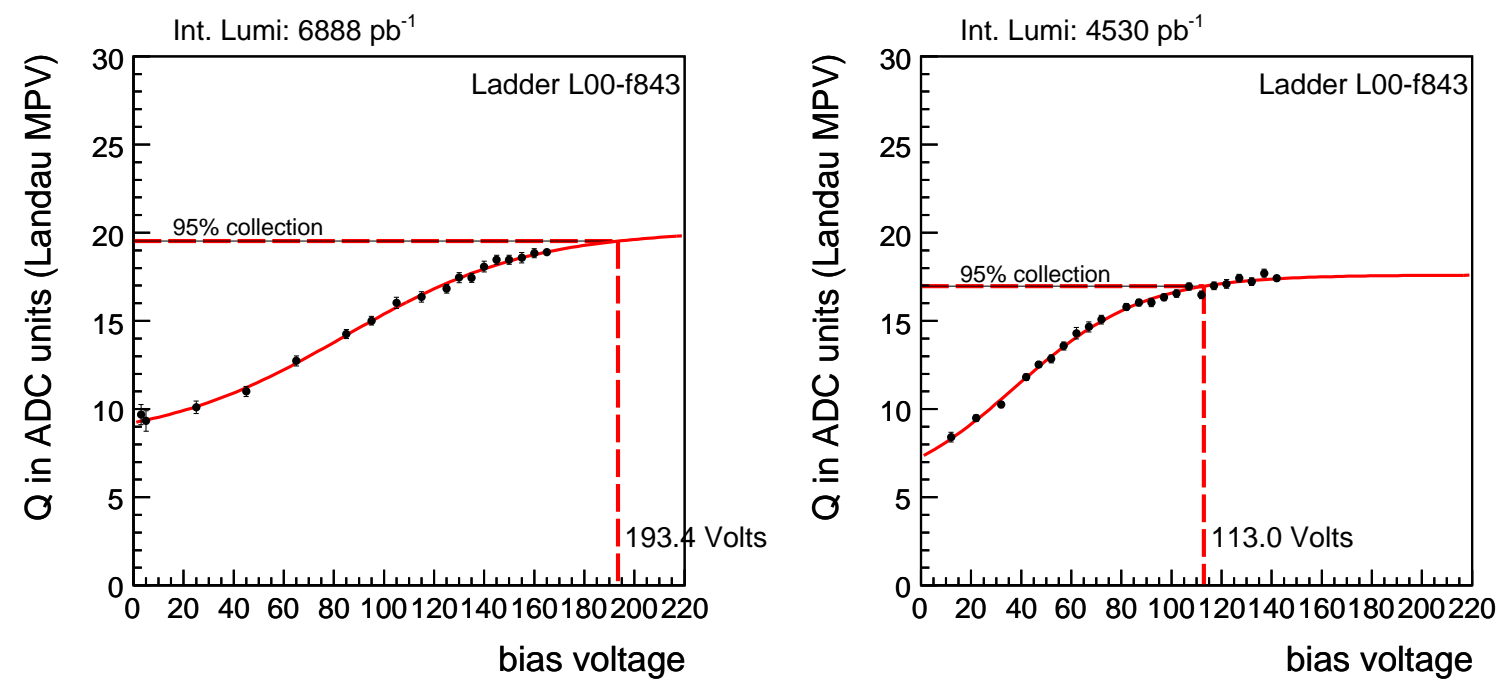

Figure A.7: Figuras de voltaje de desertización para el sensor f843 de clase SGS Thomson de L00. 

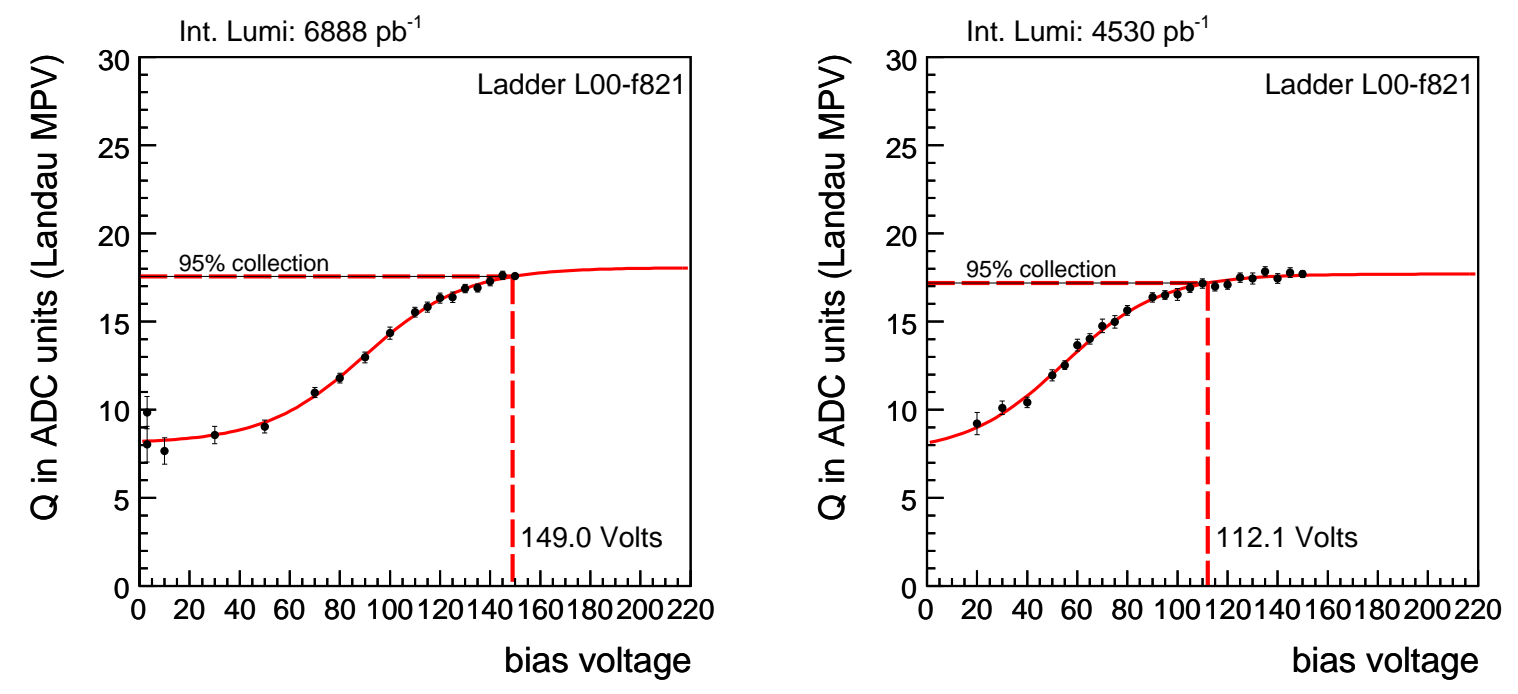

Figure A.8: Figuras de voltaje de desertización para el sensor f821 de clase Hamamatsu de L00.
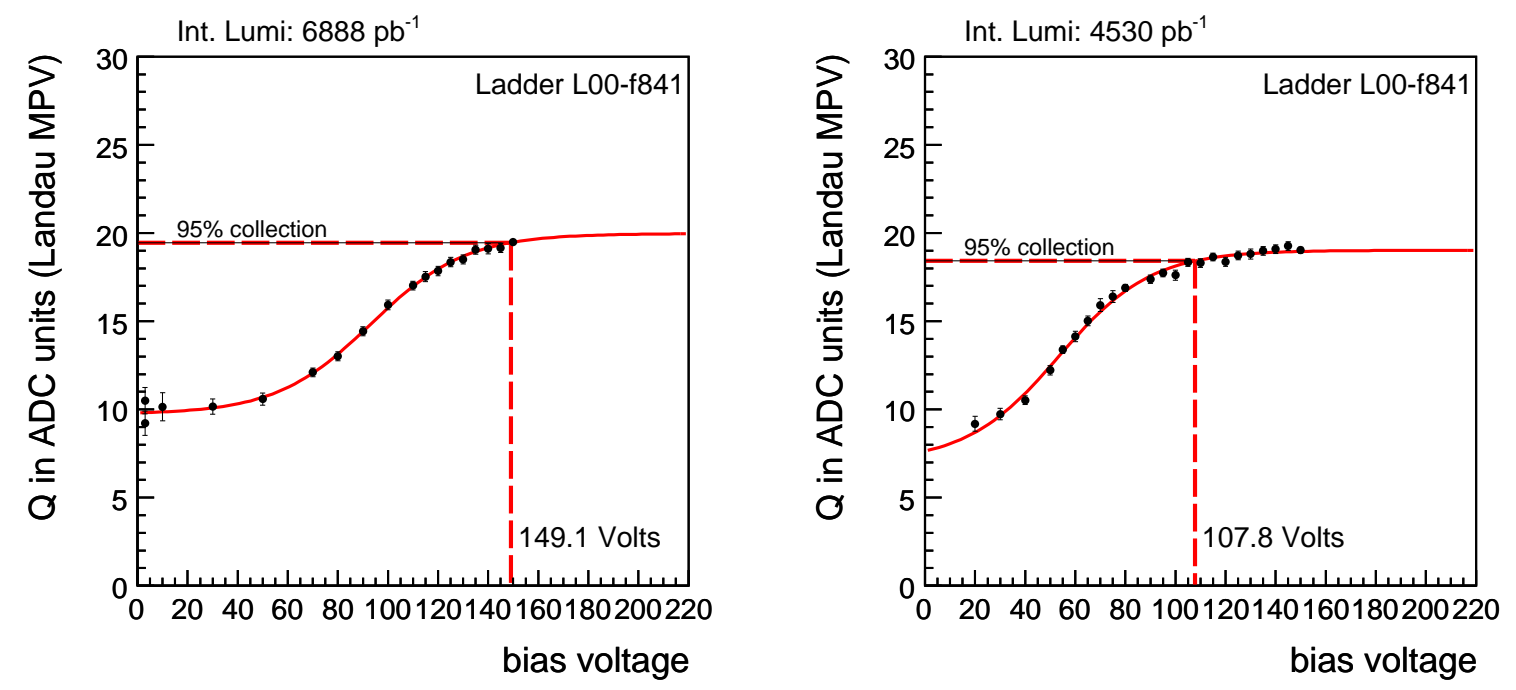

Figure A.9: Figuras de voltaje de desertización para el sensor f841 de clase Hamamatsu de L00. 


\section{A.4.1.2 Evolución del voltaje de desertización}

Para monitorizar del comportamiento de un sensor se observa la evolución de su voltaje de desertización a lo largo del tiempo.

Se han elegido sensores representativos de cada clase: Micron (Fig. A.10), SGS Thomson (Fig. A.11), y Hamamatsu (Figs. A.12 y A.13).

En esas cuatro figuras, el eje $x$ representa la luminosidad integrada a la que se realizó la prueba de señal vs. polarización. En el eje $y$ se representa el voltaje de desertización correspondiente a cada una de esas luminosidades. En este conjunto de puntos hay dos ajustes: uno, polinomial de tercer grado que ajusta los puntos a baja luminosidad, y otro, lineal, que ajusta las luminosidades más recientes. Para cada sensor se define el punto de inversión como el mínimo de la curva polinómica. Las coordenadas $(x, y)$ del punto de inversión son respectivamente la luminosidad de inversión y el voltaje de desertización de inversión. El ajuste lineal es una extrapolación de los puntos posteriores al punto de inversión.

La Figura A.14 muestra que los sensores de clase Micron resistieron más el tipo de inversión. Sus luminosidades de inversión están localizadas en torno a $2500 \mathrm{pb}^{-1}$, mientras que los sensores SGS Thomson y Hamamatsu muestran luminosidades de inversión próximas a $1500 \mathrm{pb}^{-1}$ y $1000 \mathrm{pb}^{-1}$, respectivamente. El subconjunto de puntos posterior al punto de inversión parece obedecer un comportamiento lineal para las tres clases de sensores de LOO. 

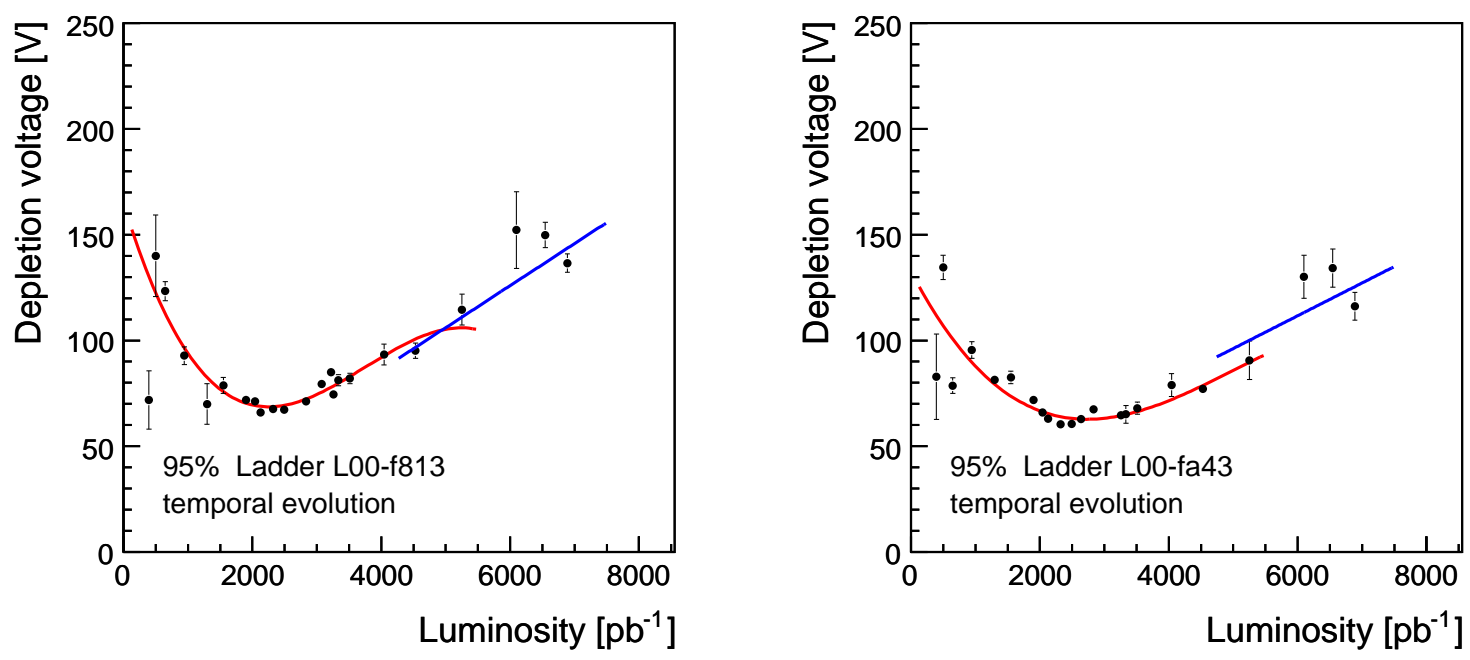

Figure A.10: Ejemplo de inversión para los dos sensores de clase Micron de L00.
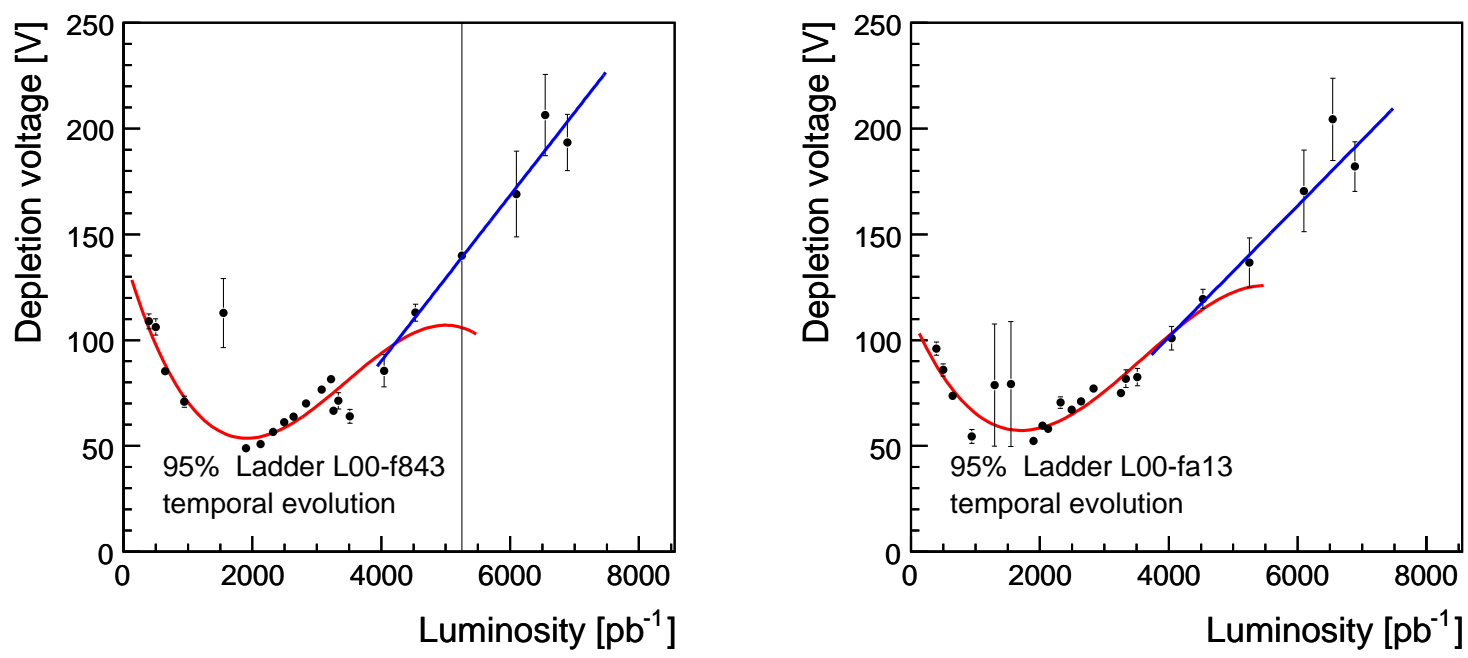

Figure A.11: Ejemplo de inversión para dos sensores de clase SGS Thomson de L00. 

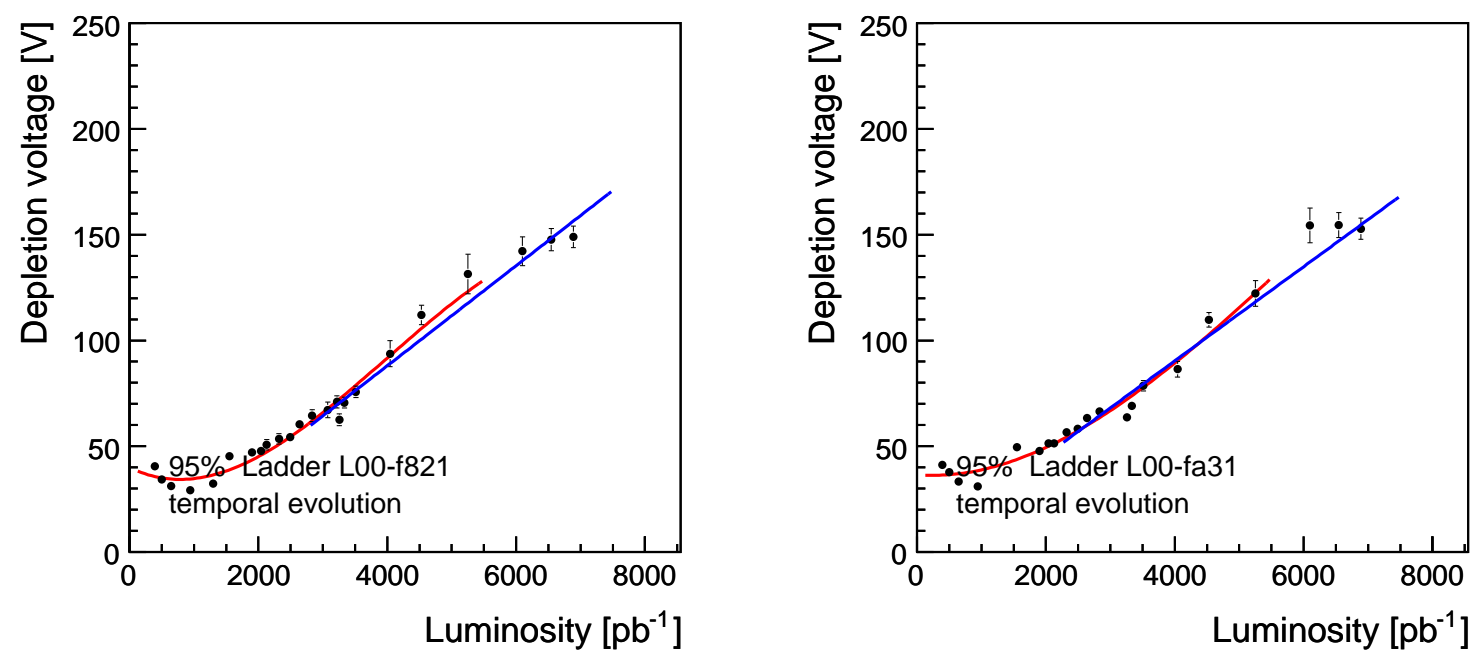

Figure A.12: Ejemplo de inversión para dos sensores de clase Hamamatsu de L00.
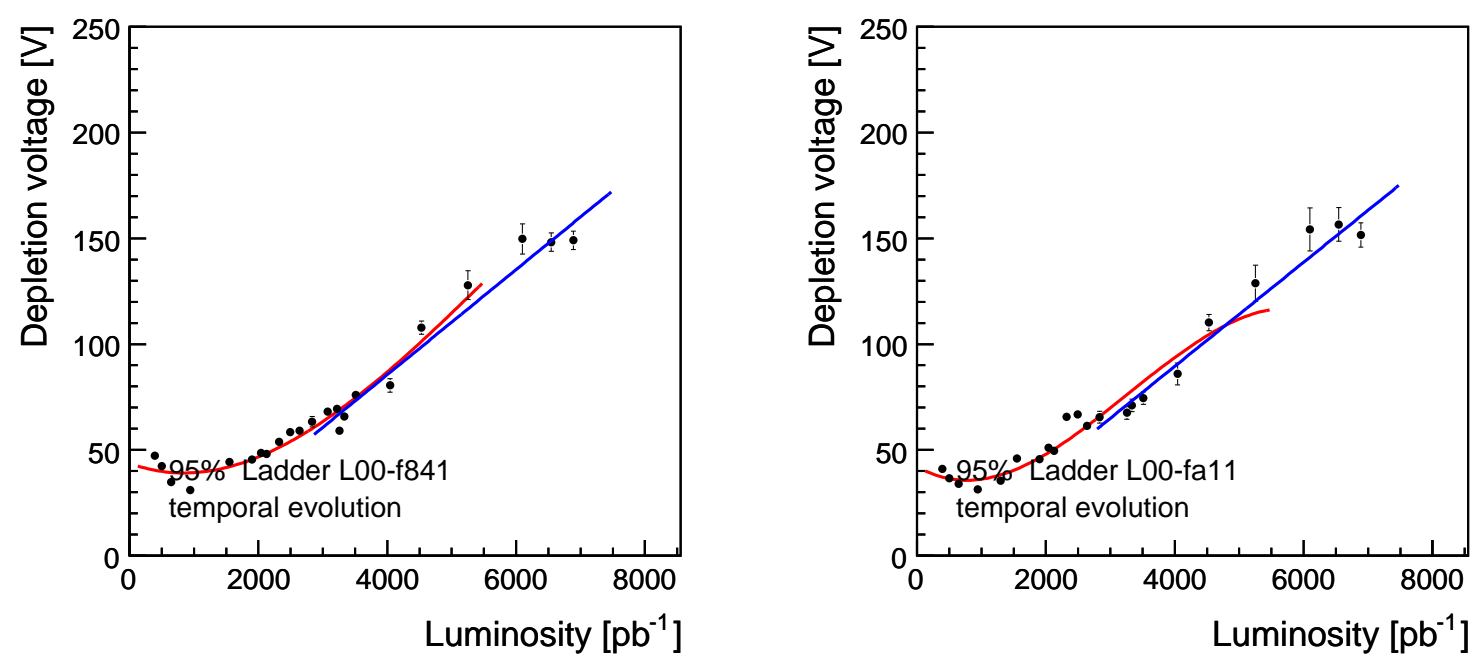

Figure A.13: Ejemplo de inversión para dos sensores de clase Hamamatsu de L00. 


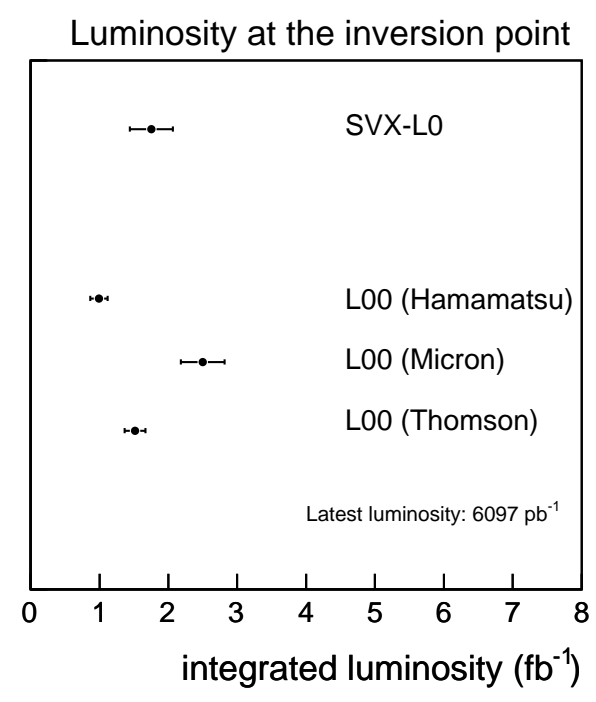

Figure A.14: Resultados generales para L00 y SVX-L0. Comparación de la luminosidad de inversión para las diferentes clases de sensores de L00 y SVX-L0. Los resultados se computaron con datos tomados a una luminosidad de $6097 \mathrm{pb}^{-1}$.

\section{A.4.1.3 Resumen de extrapolaciones lineales de LO0}

La figura resumen de extrapolaciones para L00 (Fig. A.15) muestra los ajustes lineales superpuestos de todos los 48 sensores de L00, así como los límites técnicos de la fuente de alimentación y la región de ruptura dieléctrica de los sensores. La figura A.15 compara esos ajustes lineales a dos luminosidades distintas, $6888 \mathrm{pb}^{-1}$ y $4530 \mathrm{pb}^{-1}$, para extraer información sobre la evolución de las pendientes de los ajustes. Si las pendientes a dos luminosidades distintas mostrasen diferencias significativas, la hipótesis de un comportamiento lineal posterior al proceso de inversión debería ser revisada.

En la misma figura se observa que todos los ajustes lineales están en zona segura, lejos del límite de 500 voltios y de la región de ruptura. Por tanto, con los actuales resultados se puede concluír que los sensores de LO0 pueden permanecer operativos hasta una luminosidad de al menos $10000 \mathrm{pb}^{-1}$. 

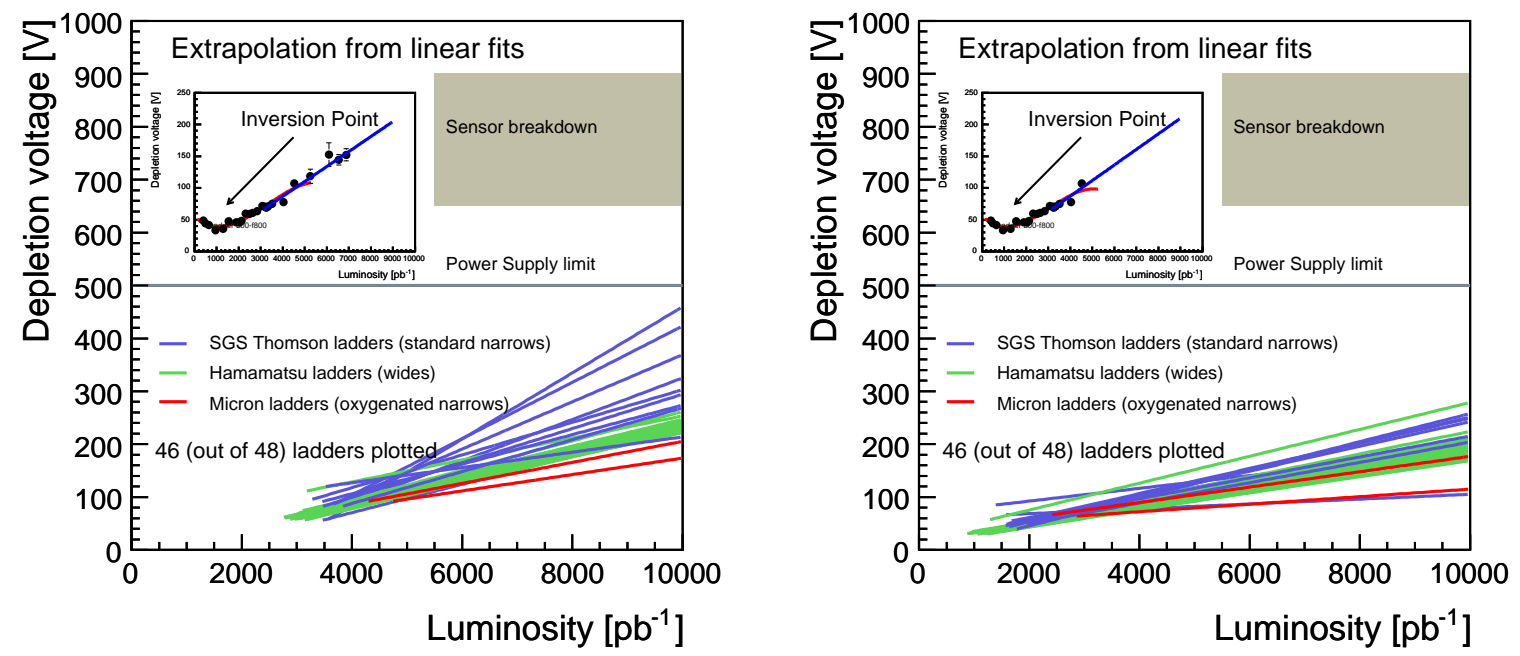

Figure A.15: Figura resumen para L00. Ajustes lineales superpuestos de todos los sensores de L00. Datos a $6888 \mathrm{pb}^{-1}$ (izquierda), y a $4530 \mathrm{pb}^{-1}$ (derecha). 


\section{A.4.2 Resultados de las exploraciones de señal vs. polarización para SVX-Lo.}

\section{A.4.2.1 Voltaje de desertización}

De manera similar al caso de L00 se muestran las figuras del voltaje de desertización para SVX-LO, con la particularidad de que todos los sensores de SVX-LO son de una única clase (Hamamatsu) y de doble cara ( $p h i, z)$. Los resultados para los sensores representativos e160 y e460 aparecen el las figuras A.16 y A.17.
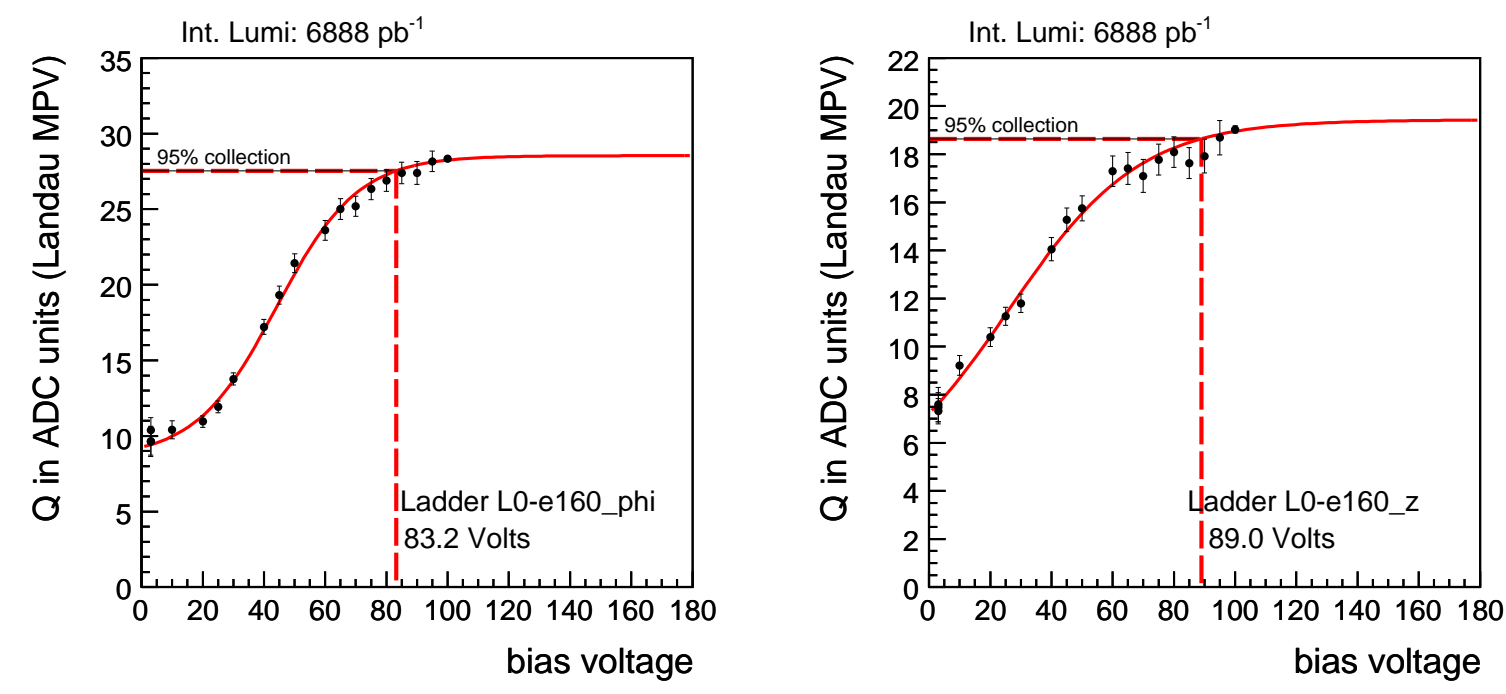

Figure A.16: Voltaje de desertización para las caras $\phi$ (izquierda) y $z$ (derecha) del sensor e160 a luminosidad de $6888 \mathrm{pb}^{-1}$. 

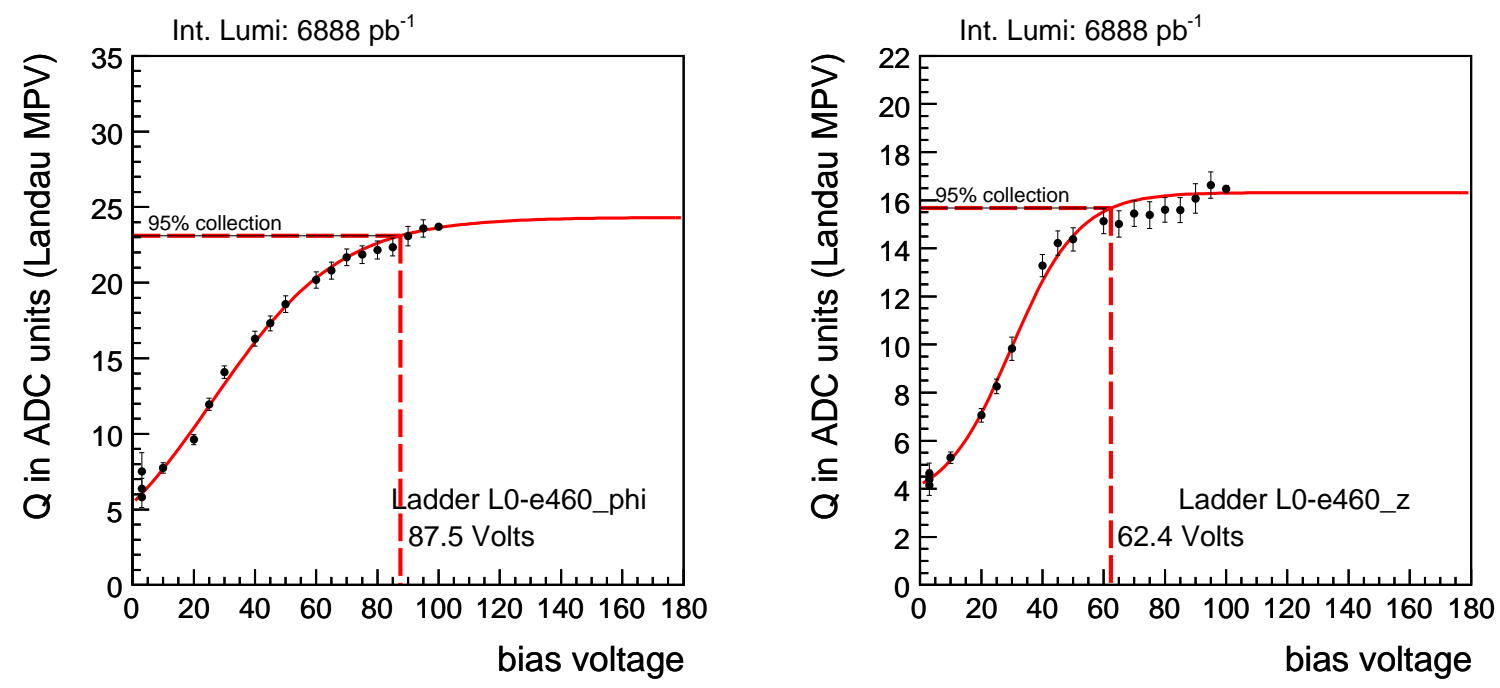

Figure A.17: Voltaje de desertización para las caras $\phi$ (izquierda) y $z$ (derecha) del sensor e460 a luminosidad de $6888 \mathrm{pb}^{-1}$. 

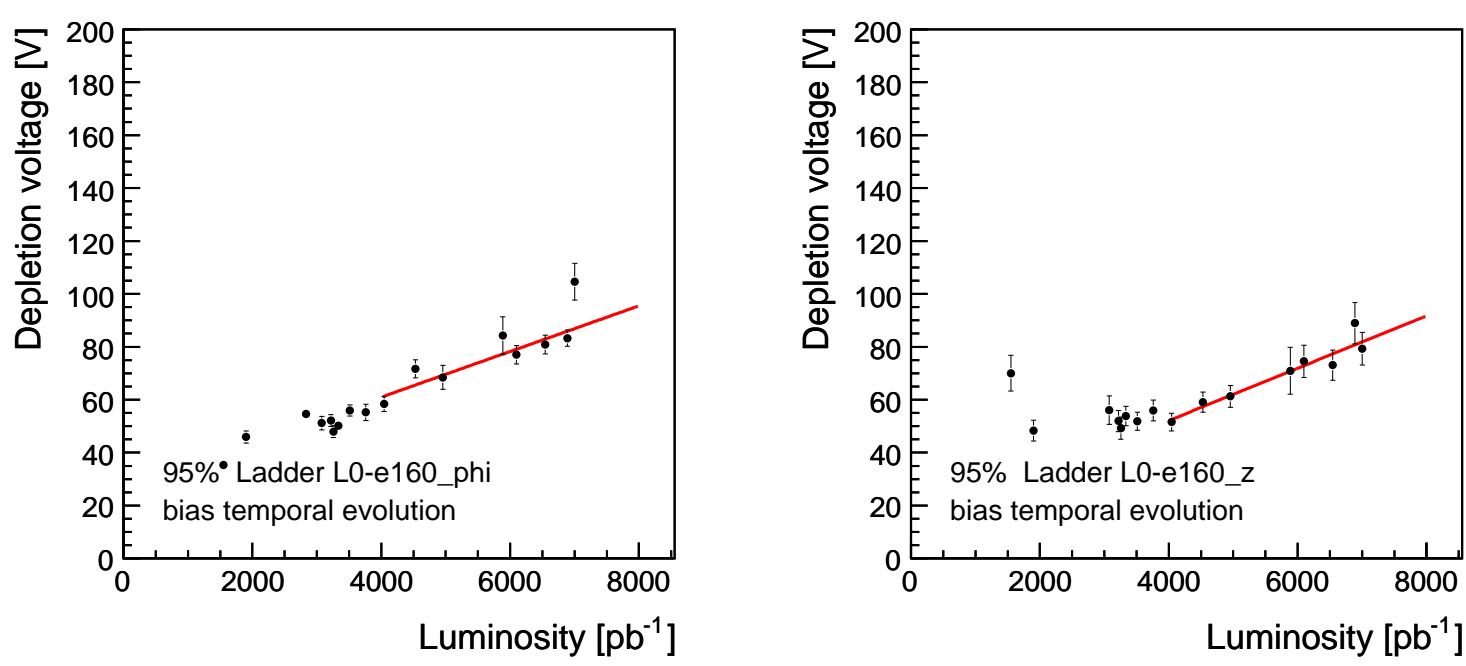

Figure A.18: Evolución histórica del voltaje de desertización del sensor e160 de SVX-L0, cara $\phi$ (izquierda) y $z$ (derecha).

\section{A.4.2.2 Evolución del voltaje de desertización}

EI sensor e160 se ha elegido para ilustrar la evolución del voltaje de desertización de SVXL0. La evolución histórica de su voltaje de desertización y carga colectada se muestra en las Figuras A.18 and A.19, respectivamente.

Para luminosidades anteriores a $3000 \mathrm{pb}^{-1}$ sólo se practicaron exploraciones de señal vs. polarización a tres (e140, e2a0, e090) de los 72 sensores de SVX-L0. A pesar de esta falta de estadística, la evolución del voltaje de desertización de esos tres sensores está cualitativamente en concordancia con el modelo (Ref. [66]) predictivo (Fig. A.20).

Observando que el subconjunto de puntos de luminosidades recientes (posteriores a $3500 \mathrm{pb}^{-1}$ ) exhibe valores crecientes, podemos asumir que todos los sensores de SVX-LO han sufrido el proceso de inversión de tipo de dopante. Un ajuste lineal a ese conjunto de puntos es útil para extraer conclusiones sobre el comportamiento futuro de SVX-LO.

\section{A.4.2.3 Resumen de extrapolaciones lineales de SVX-L0}

La figura A.21 compara la extrapolación de las caras phi de los 72 sensores de SVX-L0 a luminosidades de 6888 y $4530 \mathrm{pb}^{-1}$. La Figura A.22 es su equivalente para las caras $z$. 

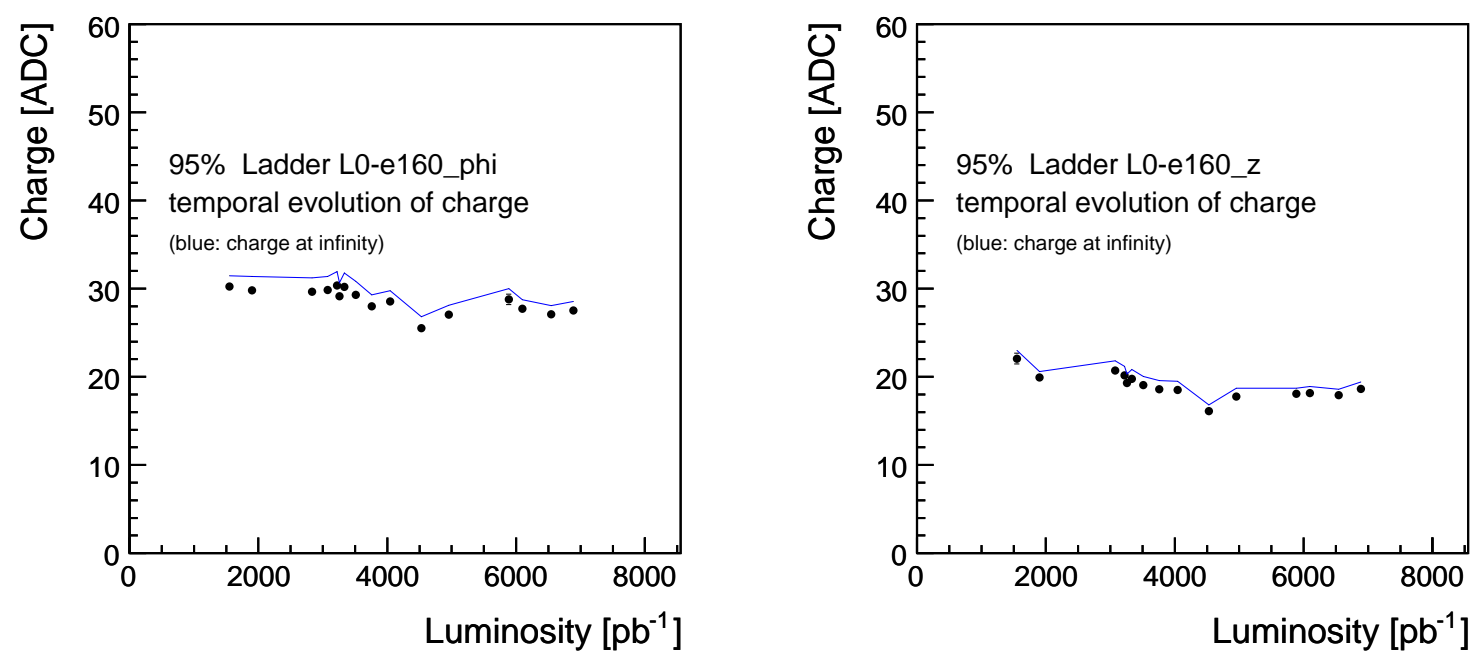

Figure A.19: Evolución histórica de la carga colectada del sensor e160 de SVX-L0, caras $\phi$ (izquierda) y $z$ (derecha).

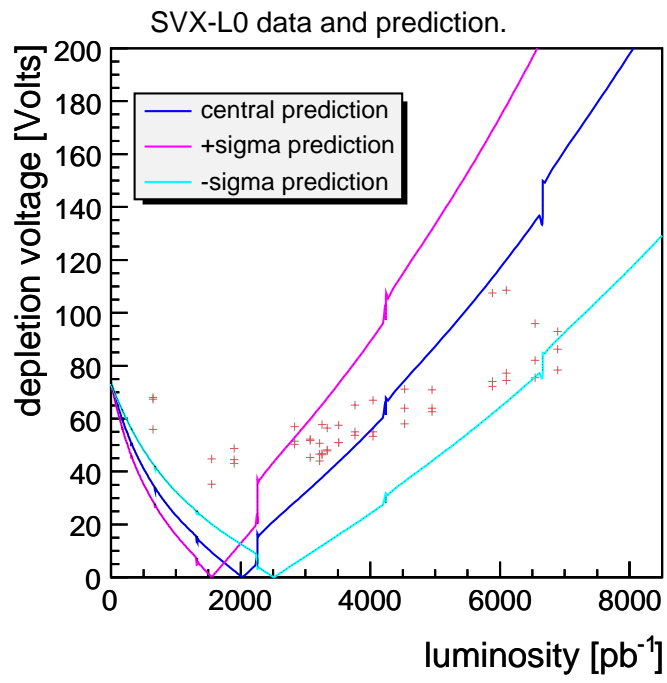

Figure A.20: Datos de evolución histórica de SVX-L0 superpuestos al modelo predictivo de inversión de tipo de dopante. Los puntos corresponden a los 3 sensores para los que existe estadística disponible a bajas luminosidades. 

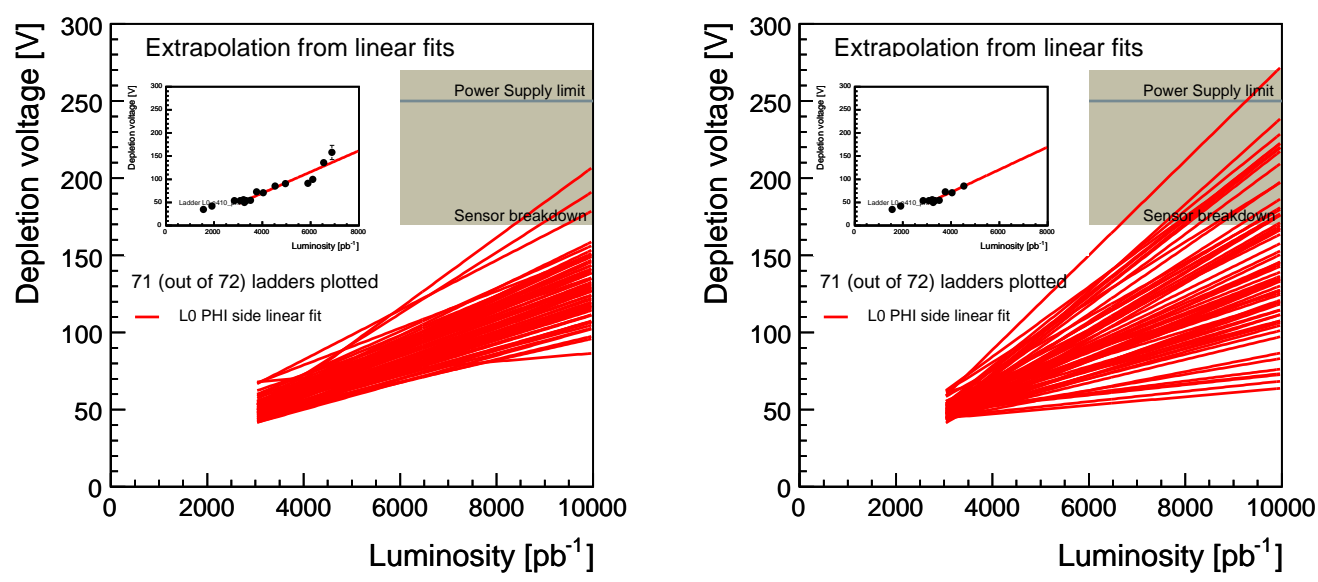

Figure A.21: Resumen de la extrapolación de los ajustes lineales para las caras phi de los sensores de SVX-L0, a $6888 \mathrm{pb}^{-1}$ (izq.) y $4530 \mathrm{pb}^{-1}$.
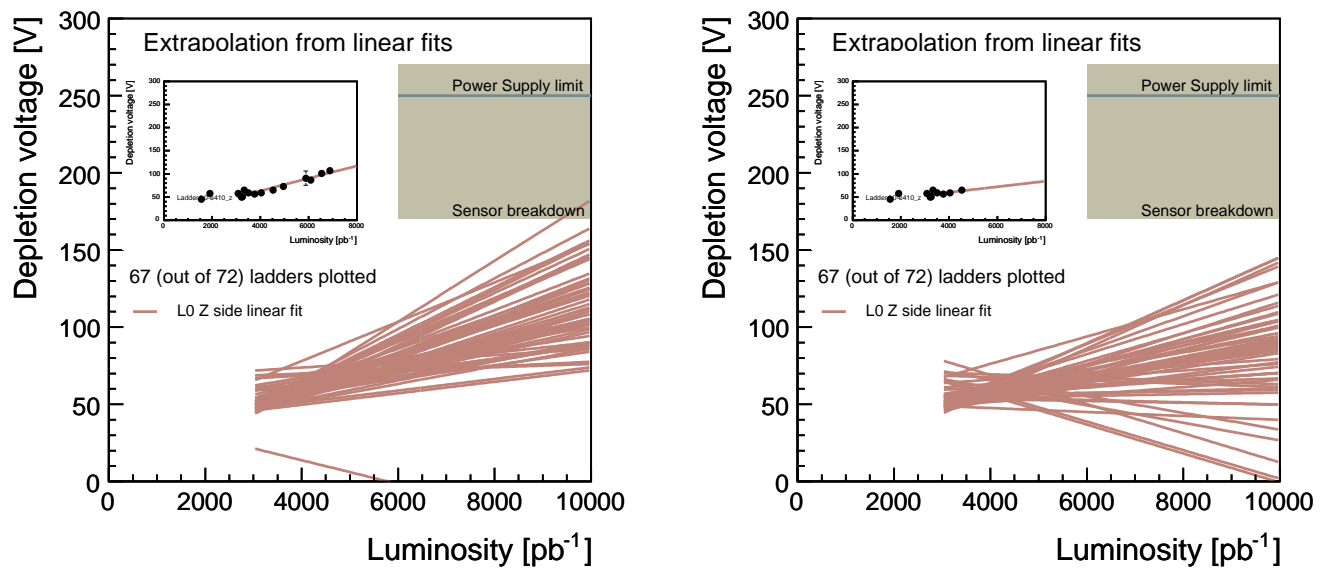

Figure A.22: Resumen de la extrapolación de los ajustes lineales para las caras $z$ de los sensores de SVX-L0, a 6888 pb $^{-1}$ (izq.) y $4530 \mathrm{pb}^{-1}$. 


\section{A.4.3 Discusión}

Las pruebas de señal vs. polarización muestran que los sensores de LO0 y de SVX-L0 han sufrido el fenómeno de inversión de tipo de dopante como consecuencia de su prolongada exposición a la radiación (Fig. A.14).

Los sensores SGS Thomson de L00 invirtieron a luminosidades próximas a $1500 \mathrm{pb}^{-1}$. Los sensores Hamamatsu de LO0 y de SVX-L0 lo hicieron a $1000 \mathrm{pb}^{-1}$ y $1700 \mathrm{pb}^{-1}$ respectivamente. Esta diferencia tiene su explicación en la dependencia radial de ambos dispositivos frente al campo de radiación [68], [66]. Para L00, el hecho de que la mayor luminosidad de inversión corresponda a los sensores de clase Micron es de importancia para el experimento CMS del LHC cuyos detectores de silicio utilizan la misma tecnología resistente a la radiación en sus sensores.

Una de las cuestiones principales observable en los datos de tipo de inversión es la discordancia entre los datos experimentales y el modelo, tal y como se muestra en la Figura A.20. El modelo predictivo asume que la inversión ocurre de manera homogénea en todo el perfil de espesor del sensor (Figura A.23). En este caso, el mínimo del punto de inversión (voltaje de desertización de inversión) es cero voltios. Sin embargo los datos muestran que, dependiendo de cada clase de sensor, el mínimo está situado entre los 30 y 50 voltios. Descartando caídas de voltaje en el circuito de polarización, esta discrepancia podría explicarse haciendo uso de modelos de perfil de dopaje no homogéneo como por ejemplo el de doble unión $p n$ (Figura A.24, Ref. [81]). 


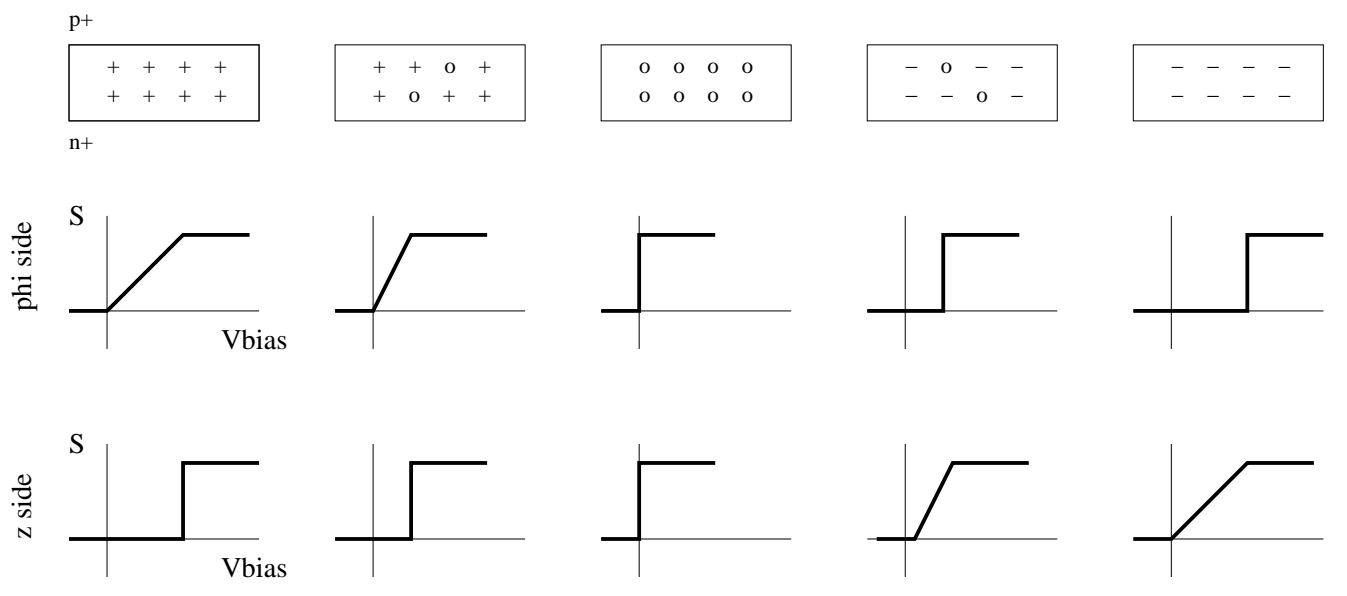

Figure A.23: Espacio de carga de un sensor de SVX-L0 ideal (perfil de dopante homogéneo) y comportamiento frente a luminosidad de la curva de señal vs. polarización.

\section{A.5 Conclusiones}

Este trabajo ha sido dedicado al estudio de los efectos provocados por la radiación que han sido observados en los detectores de silicio de CDF, en particular, la inversión de tipo de dopante y la evolución de las corrientes de polarización.

Los detectores de silicio de CDF fueron diseñados para ser sustituido después de 2-3 fb ${ }^{-1}$ de datos registrados. Sin embargo, la sustitución prevista no fue posible y los sensores han debido resistir hasta 10-12 $\mathrm{fb}^{-1}$. Esta circunstancia particular ha sido un reto para la operación y mantenimiento de los detectores de silicio y la razón detrás del minucioso trabajo de seguimiento de los que han sido objeto.

El daño por radiación se ha estudiado desde dos puntos de vista: la corriente de polarización y la tensión de polarización. También se han observado y registrado otros efectos debido a daño por radiación y envejecimiento sobre diversos parámetros del detector (eficiencia, tamaño del clúster ...).

El estudio de las corrientes de polarización es relevante en un sensor de silicio irradiado ya que es un parámetro relacionado con el flujo de energía recibida. Por lo tanto, y mediante el estudio de los datos de corrientes, es posible relacionar la luminosidad, una cantidad conocida, con la dosis total recibida por el detector. Los resultados expresados como función de la fluencia son independiente del entorno local (i.e. el Tevatron) y pueden ser comparados con resultados de otros experimentos.

En cuanto a la tensión de polarización, su evolución a lo largo del tiempo evidencia el 


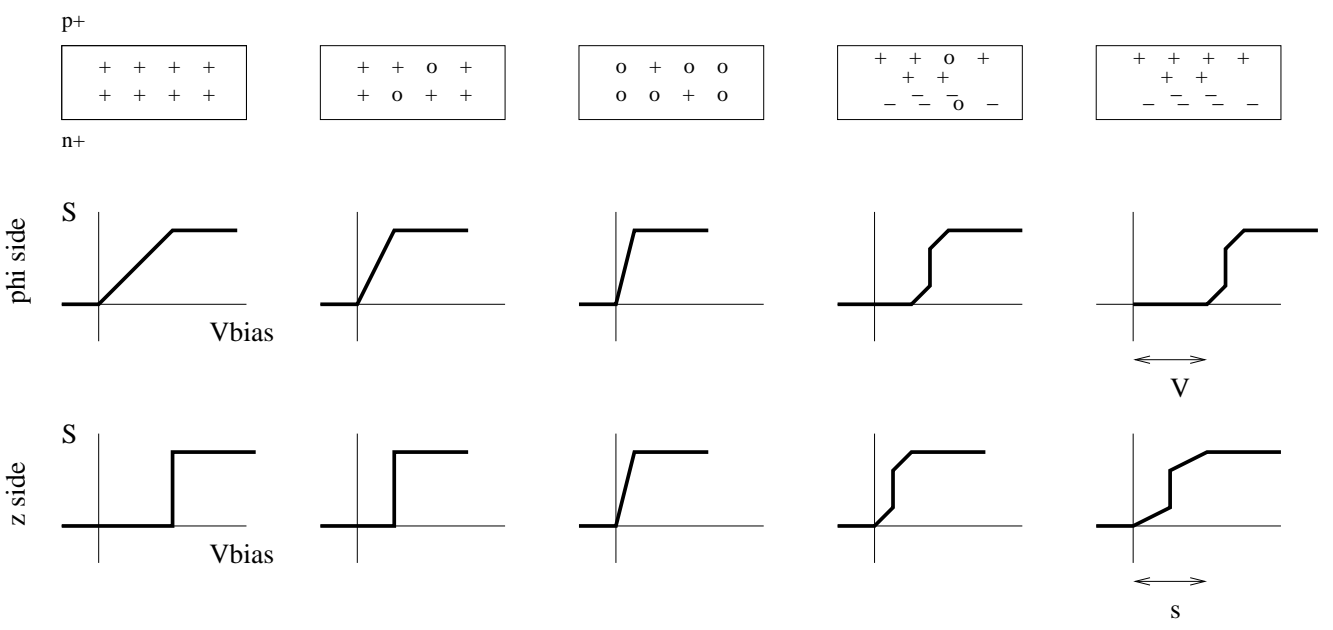

Figure A.24: Espacio de carga de un sensor de SVX-L0 real (perfil de dopante inhomogéneo, caso de lineal simple) y comportamiento frente a luminosidad de la curva de señal vs. polarización. La presencia de la pendiente $s$ observada en las curvas experimentales podría evidenciar una estructura subyacente de doble unión (Apéndice D).

fenómeno de inversión para L00 y SVX-LO. Dado que los detectores de silicio son el factor limitante en la vida del experimento CDF, las extrapolaciones de los ajustes lineales de los puntos posteriores a la inversión han sido la referencia para las inspecciones periódicas de los comisionados del Departamento de Energía de EE.UU. y los informes sobre el presupuesto anual.

La importancia científica del fenómeno de inversión de tipo de dopante se hace evidente dado que los actuales datos experimentales no concuerdan perfectamente con el actual modelo predictivo (modelo de Hamburgo). En este modelo, el mínimo de la curva de inversión se reduce a cero voltios, mientras que la curva de inversión de los datos de CDF muestra voltaje mínimo que se encuentra entre los 30 y los 45 voltios. Dado que la resistencia interna del circuito de polarización contribuiría con una caída de voltaje del orden de 10 voltios, el resto de voltaje observado no puede explicarse con el actual modelo. El fenómeno de inversión de tipo de dopante también se ha ilustrado -aunque con menos precisión- desde el punto de vista del perfil de dopaje a bajos voltajes de polarización. Aunque algunos complejos creados en el interior del volumen del detector son bien conocidos y capaces de explicar los resultados macroscópicos, los mecanismos más profundos detrás del tipo de inversión son todavía muchos y desconocidos. La inversión de tipo de dopante es todavía una cuestión abierta en las áreas de ciencia de materiales y física del estado sólido.

Los resultados recogidos en este trabajo han sido mostrados al público en varias charlas y reuniones entre 2008 y 2009: 
* "Longevity and Radiation Aging Studies of the CDF II Silicon Detectors", charla en American Physical Society Meeting, Saint Louis MO, Abril 2008.

* "Longevity Studies of the CDF II Silicon Detectors", charla en el CMS Symposium, Fermilab, Noviembre 2008.

* "Longevity Studies of the CDF II Silicon Detectors", charla en el CIEMAT High Energy Physics Christmas Workshop, Madrid, Diciembre 2008.

* "Longevity Studies of the CDF II Silicon Detectors", charla en el All Experimenters Meeting, Fermilab, Enero 2009.

* "The CDF RUN II Silicon Detectors: Longevity Studies", poster en el 11th Pisa Meeting on Advanced Detectors, Elba, Mayo 2009.

Una descripción detallada del comportamiento de los detectores de silicio de CDF será publicada por Nuclear Instruments and Methods A, con el título "Operational Experience, Improvements, and Performance of the CDF Run II Silicon Vertex Detector". 

Appendix B

The diode Equation 


\section{B.1 Carrier concentrations in pure semiconductors}

To avoid confusion with exponential functions, the symbol $q_{e}$ will be used for the electronic charge instead of $e$. In thermal equilibrium the probability that an electron state in the conduction band is filled is given by the Fermi-Dirac distribution

$$
f_{e}(E)=\frac{1}{e^{\left(E-E_{F}\right) / k T}+1} .
$$

where the parameter $E_{F}$ is the Fermi level or chemical potential. The density of atoms in a Si or Ge crystal is about $5 \cdot 10^{22}$ atoms $/ \mathrm{cm}^{3}$. Since the minimum carrier density of interest in practial devices is of order of $10^{10}$ to $10^{11}$ atoms $/ \mathrm{cm}^{-3}$, very small occupancies are quite important.

In silicon the bandgap is $\mathbf{1 . 1 2} \mathbf{~ e V}$. If the Fermi level is at midgap, the bandedges will be $0.56 \mathrm{eV}$ above and below $E_{F}$. From the structure of the Fermi-Dirac equation, relatively large deviations from the Fermi level (i.e. extremely small occupancies) will still yield significant carrier densities.

The number of occupied electron states $N_{e}$ is determined by summing over all available states multiplied by the occupation probability for each individual state

$$
N_{e}=\sum_{i} m_{i} f\left(E_{i}\right)
$$

Since the density of states near the band edge tends to be quite high, this can be written as an integral

$$
N_{e}=\int_{E_{c}}^{\infty} f(E) g(E) d E
$$

where $g(E)$ is the density of states. Solution of this integral requires knowledge of the density of states. Fortuitously, to a good approximation the density of states near the band edge has a parabolic distribution

$$
g(E) d E \propto\left(E-E_{c}\right)^{1 / 2}
$$

As the energy increases beyond the band edge, the distribution will deviate from the simple parabolic form, but since the probability function decreases very rapidly, the integral will hardly be affected. The second obstacle to a simple analytical solution of the integral is the intractability of integrating over the Fermi distribution. Fortunately, if $E-E_{F}$ is at least several times $k T$, the Fermi distribution can be approximated by a Boltzmann distribution:

$$
\text { if } 1+e^{\left(E-E_{F}\right) / k T} \approx e^{\left(E-E_{F}\right) / k T} \Rightarrow f(E) \approx e^{\left(E-E_{F}\right) / k T}
$$


At energies $2.3 k T$ beyond the Fermi level the difference between the Boltzmann approximation and the Fermi distribution is $<10 \%$, and for energies $>4.5 k T$ it is less than $1 \%$.

Applying the approximation to the occupancy of hole states, the probability of a hole state being occupied, i.e. a valence state being empty, is

$$
f_{h}(E)=1-f_{e}(E)=\frac{1}{e^{\left(E_{F}-E\right) / k T}+1} \approx e^{-\left(E_{F}-E\right) / k T} .
$$

The conditions for the Boltzmann approximation are fulfilled for excitation across the bandgap, as the bandgap is of order $1 \mathrm{eV}$ and $k T$ at room temperature is $0.026 \mathrm{eV}$. With these simplifications the number of electrons in the conduction band $\left(n_{e}=n\right)$ in thermal equilibrium is

$$
n_{e} \propto(k T)^{3 / 2} e^{-\left(E_{c}-E_{F}\right) / k T}
$$

or

$$
n_{e}=N_{c} e^{-\left(E_{c}-E_{F}\right) / k T}
$$

where $N_{c}$ is the effective density of states at the band edge. Correspondingly, the hole concentration is

$$
p=N_{v} e^{-\left(E_{F}-E_{v}\right) / k T}
$$

In an ideal semiconductor the only source of mobile carriers is thermal excitation across the bandgap (additional impurity atoms or crystal imperfections that would allow other excitation mechanisms are absent), so the concentrations of electrons and holes are equal

$$
n=p=n_{i}
$$

where $n_{i}$ is called the intrinsic carrier concentration. In silicon $\left(E_{g}=1.12 \mathrm{eV}\right)$ the intrinsic concentration $n_{i}=1.45 \cdot 10^{10} \mathrm{~cm}^{-3}$ at $300 \mathrm{~K}$ and in germanium $\left(E_{g}=0.66 \mathrm{eV}\right), n_{i}=2.4$. $10^{13} \mathrm{~cm}^{-3}$. For comparison, the purest semiconductor material that has been fabricated is Ge with active impurity levels of about $3 \cdot 10^{10} \mathrm{~cm}^{-3}$.

Using the above results

$$
n_{i}=N_{c} e^{-\left(E_{c}-E_{F}\right) / k T}=N_{v} e^{-\left(E_{F}-E_{v}\right) / k T}
$$

and solving

$$
E_{F}=E_{i}=\frac{E_{c}+E_{v}}{2}-\operatorname{frack} T 2 \log \left(N_{c} / N_{v}\right)
$$

If the band structure is symmetrical $\left(N_{c}=N_{v}\right)$, the intrinsic energy level $E_{i}$ lies near the middle of the bandgap. Even rather substantial deviations from a symmetrical band 
structure will not affect this result significantly, as $N_{c} / N_{v}$ enters logarithmically and $k T$ is much smaller than the bandgap.

A remarkable result is that the product of the electron and hole concentrations:

$$
n p=n_{i}^{2}=N_{c} N_{v} e^{-\left(E_{c}-E_{v}\right) / k T}=N_{c} N_{v} e^{-E_{g} / k T}
$$

depends only on the bandgap $E_{g}$ and not on the Fermi level. This result, the law of mass action, is very useful in semiconductor device analysis. It requires only that the Boltzmann approximation holds. Qualitatively, it says that if one carrier type exceeds this equilibrium concentration, recombination will decrease the concentrations of both electrons and holes to maintain $n p=n_{i}^{2}$, a relationship that also holds in doped crystals.

\section{B.2 Carrier concentrations in doped crystals}

The equality $n_{e}=n_{h}$ only holds for pure crystals, where all of the electrons in the conduction band have been thermally excited from the valence band. In practical semiconductors the presence of impurities tips the balance towards either electrons or holes.

Impurities are an unavoidable byproduct of the crystal growth process, although special techniques can achieve astounding results. For example, as noted above, in the purest semiconductor crystals (high purity Ge) the net impurity concentration is about $3 \cdot 10^{10}$ $\mathbf{c m}^{-3}$.

In semiconductor device technology impurities are introduced intentionally to control the conductivity of the semiconductor. Let $N_{d}^{+}$be the concentration of ionized donors and $N_{a}^{-}$the concentration of ionized acceptors. Overall charge neutrality is preserved, as each ionized dopant introduces a charged carrier and an oppositely atom, but the net carrier concentration is now

$$
\Delta n=n-p=N_{d}^{+}-N_{a}^{-}
$$

or

$$
p+N_{d}^{+}=n+N_{a}^{-} .
$$

Assume that the activation energy of the donors and acceptors is sufficiently small so that they are fully ionized. Then $N_{d}^{+}=N_{d}$ and $N_{a}^{-}=N_{a}$, so

$$
p+N_{d}=n+N_{a},
$$

which using $n p=n_{i}^{2}$, becomes

$$
p+N_{d}=\frac{n_{i}^{2}}{p}+N_{a},
$$




$$
\frac{p}{N_{a}}+\frac{N_{d}}{N_{a}}=\frac{n_{i}}{p} \frac{n_{i}}{N_{a}}+1
$$

If the acceptor concentration $N_{a} \gg N_{d}$ and $N_{a} \gg n_{i}$, the hole and electron concentrations

$$
p \approx N_{a} \text { and } n \approx \frac{n_{i}^{2}}{N_{a}} \ll N_{a}
$$

i.e. the conductivity is dominated by holes. Conversely, if the donor concentration $N_{d} \gg$ $N_{a}$ and $N_{d} \gg n_{i}$ the conductivity is dominated by electrons.

If the conductivity is dominated by only one type of carrier, the Fermi level is easy to determine. If, for example $n \gg p$, then equation B.16 can be written as

$$
\begin{aligned}
n & =N_{d}-N_{a} \\
N_{c} e^{-\left(E_{c}-E_{F}\right) / k T} & =N_{d}-N_{a},
\end{aligned}
$$

yielding

$$
\frac{E_{c}-E_{F}}{k_{B} T}=\log \left(\frac{N_{c}}{N_{d}-N_{a}}\right)
$$

If $N_{d} \gg N_{a}$, then $E_{c}-E_{F}$ must be small, i.e. the Fermi level lies close to the conduction band edge.

In reality the impurity levels of common dopants are not close enough to the band edge for the Boltzmann approximation to hold, so the calculation must use the Fermi distribution and solve numerically for $E_{F}$. Nevertheless, the qualitative conclusions derived here still apply.

It is often convenient to refer all of these quantities to the intrinsic level $E_{i}$, as it accounts for both $E_{c}$ and $E_{v}$. Then

$$
\begin{aligned}
& n=N_{c} e^{-\left(E_{c}-E_{F}\right) / k T}=n_{i} e^{-\left(E_{F}-E_{i}\right) / k T} \\
& p=N_{v} e^{-\left(E_{F}-E_{v}\right) / k T}=n_{i} e^{-\left(E_{i}-E_{F}\right) / k T}
\end{aligned}
$$

and the Fermi level

$$
E_{F}-E_{i}=-k_{B} T \log \frac{N_{a}-N_{d}}{n_{i}}
$$




\section{B.3 pn-junctions}

A $p n$-junction is formed at the interface of a $p$ - and $n$-type region. Since the electron concentration in the $n$-region is greater than in the $p$-region, electrons will diffuse into the $p$-region. Correspondingly, holes will diffuse into the $n$-region. As electrons and holes diffuse across the junction, a space charge due to the ionized donor and acceptor atoms builds up. The field due to this space charge is directed to impede the flow of electrons and holes.

The situation is dynamic. The concentration gradient causes a continuous diffusion current to flow, whereas the field due to the space charge drives a drift current in the opposite direction. Equilibrium is attained when the two currents are equal, i.e. the sum of the diffusion and drift current is zero. The net hole current density is

$$
J_{p}=-q_{e} D_{p} \frac{d p}{d x}+q_{e} p \mu_{p} E_{p}
$$

where $D_{p}$ is the diffusion constant for holes and $E_{p}$ is the electric field in the $p$-region.

To solve this equation we make use of the following relationships: the hole concentration is

$$
p=n_{i} e^{\left(E_{i}-E_{F}\right) / k T},
$$

and its derivative

$$
\frac{d p}{d x}=\frac{p}{k T}\left(\frac{d E_{i}}{d x}-\frac{d E_{F}}{d x}\right)
$$

Since the force on a charge $q_{e}$ due to an electric field $E$ is equal to the negative gradient of the potential energy,

$$
q_{e} E=-\frac{d E_{c}}{d x}=-\frac{d E_{v}}{d x}=-\frac{d E_{i}}{d x} .
$$

As only the gradient is of interest and $E_{c}, E_{v}$, and $E_{i}$ differ only by a constant offset, any of these measures can be used. We will use the intrinsic Fermi level $E_{i}$, since it applies throughout the sample.

The remaining expression is the Einstein relationship, which relates the mobility to the diffusion constant:

$$
\mu_{p}=\frac{q_{e} D_{p}}{k T} .
$$

Using these relationships the net hole an electron currents become:

$$
\begin{gathered}
J_{p}=q_{e} p \frac{D_{p}}{k T} \frac{d E_{F}}{d x}=\mu_{p} p \frac{d E_{F}}{d x} . \\
J_{n}=-q_{e} n \frac{D_{n}}{k T} \frac{d E_{F}}{d x}=-\mu_{n} n \frac{d E_{F}}{d x} .
\end{gathered}
$$


Since, individually, the net hole and electron currents in equilibrium must be zero, the derivative of the Fermi level:

$$
\frac{d E_{F}}{d x}=0
$$

In thermal equilibrium the Fermi level must be constant throughout the junction region. For the Fermi level to be flat, the band structure must adapt, since on the $p$-side the Fermi level is near the valence band, whereas on the $n$-side it is near the conduction band. If we assume that the dopants are exclusively donors on the $n$-side and acceptors on the $p$-side, the difference in the respective Fermi level is

$$
\Delta E_{F}=-k T \log \frac{N_{a} N_{d}}{n_{i}^{2}} .
$$

This corresponds to an electric potential

$$
\Delta V_{F}=\frac{1}{q_{e}} \Delta E_{F} \equiv V_{b i}
$$

often referred to as the "buil-in" voltage of the junction.

As either $N_{a}$ or $N_{d}$ increases relative to $n_{i}$, the respective Fermi level moves closer to the band edge, increasing the built-in voltage. With increasing doping levels the built-in voltage approaches the equivalent potential of the bandgap $E_{g} / q_{e}$.

\section{B.4 The forward-biased pn-junction}

Applying an external bias leads to a condition that deviates from thermal equilibrium, i.e. the Fermi level is no longer constant throughout the junction. If a positive voltage is applied to the $p$-electrode relative to the $n$-electrode, the total variation of the electric potential across the junction will decrease. Since this reduces the electric field across the junction, the drift component of the junction current will decrease. Since the concentration gradient is uncharged, the difusion current will exceed the drift current and a net current will flow.

This net current leads to an excess of electrons in the $p$-region and an excess of holes in the $n$-region. This "injection" condition leads to a local deviation from equilibrium, i.e.pn > $n_{i}^{2}$. Equilibrium will be restored by recombination.

Note that a depletion region exists even under forward bias, although its width is decreased. The electric field due to the space charge opposes the flow of charge, but the large concentration gradient overrides the field.

Consider holes flowing into the $n$-region. They will flow through the depletion region with small losses due to recombination, as the electron concentration is small compared with 
the bulk. When holes reach the $n$-side boundary of the depletion region the concentration of electrons available for recombination increases and the concentration of holes will decrease with distance, depending on the cross-section for recombination, expressed as a diffusion length. Ultimately, all holes will have recombined with electrons. The required electrons are furnished through the external contact from the power supply.

On the $p$-side, electrons undergo similar process. The holes required to sustain recombination are formed at the external contact to the $p$-region by electron flow toward the power supply, equal to the electron flow toward the $n$-contact. The following derivation follows the discussions by Shockley $(1949,1950)$ and Grove $(1967)$.

The steady-state distribution of charge is determined by solving the diffusion equation,

$$
D_{n} \frac{d^{2} n_{p}}{d x^{2}}-\frac{n_{p}-n_{p 0}}{\tau_{n}}=0
$$

Electrons flowing into the $p$-region give rise to a local concentration $n_{p}$ in excess of the equilibrium concentration $n_{p 0}$. This excess will decay with recombination time $\tau_{n}$, corresponding to a diffusion length $L_{n}$.

The first boundary condition required for the solution of the diffusion equation is that the excess concentration of electrons vanish at large distances $x$,

$$
n_{p}(\infty)=n_{p 0}
$$

The second boundary condition is that the carriers are injected at the origin of the space charge region $x=0$ with a concentration $n_{p}(0)$. This yields the solution

$$
n_{p}(x)=n_{p 0}+\left(n_{p}(0)-n_{p 0}\right) e^{x / L_{n}}
$$

From this we obtain the electron current entering the $p$-region

$$
J_{n p}=-\left.q_{e} D_{n} \frac{d n_{p}}{d x}\right|_{x=0}=q_{e} D_{n} \frac{n_{p}(0)-n_{p 0}}{L_{n}} .
$$

This says that the electron current is limited by the concentration gradient determined by the carrier density at the depletion edge $n_{p}(0)$ and the equilibrium minority carrier density $n_{p 0}$. Determining the equilibrium density $n_{p 0}$ is easy,

$$
n_{p 0}=n_{i}^{2} / N_{a}
$$

The problem is that $n_{p 0}$ is established in a non-equilibrium state, where the previously employed results do not apply. To analyze the regions with non-equilibrium carrier concentrations, Shockley introduced a simplifying assumption by postulating that the product $p n$ is 
constant. In this specific quasi-equilibrium state this constant will be larger than $n_{i}^{2}$, the $p n$ product in thermal equilibrium. In analogy to thermal equilibrium, this quasi-equilibrium state is expressed in terms of a "quasi-Fermi level", which is the quantity used in place of $E_{F}$ that gives the carrier concentration under non-equilibrium conditions.

The postulate $p n=$ const is equivalent to stating that the non-equilibrium carrier concentration are given by a Boltzmann distribution, so the concentration of electrons is

$$
n=n_{i} e^{\left(E_{F n}-E_{i}\right) / k T},
$$

where $E_{F n}$ is the quasi-Frmi level for electrons, and

$$
p=n_{i} e^{\left(E_{i}-E_{F p}\right) / k T},
$$

where $E_{F p}$ is the quasi-Frmi level for holes. The product of the two carrier concentrations in non-equilibrium is:

$$
p n=n_{i}^{2} e^{\left(E_{F n}-E_{F p}\right) / k T} .
$$

If $p n$ is constant throughout the space-charge region, then $E_{F n}-E_{F p}$ must also remain constant.

Using the quasi-Fermi level and the Einstein relationship, the electron current entering the $p$-region becomes

$$
J_{n p}=-\left.q_{e} D_{n} \frac{d n_{p}}{d x}\right|_{x=0}=-q_{e} D_{n} \frac{d}{d x}\left(n_{i} e^{\left(E_{F n}-E_{i}\right) / k_{B} T}\right)=-\mu_{n} n \frac{d E_{F n}}{d x} .
$$

These relationships describe the behavior of the quasi-Fermi level in the depletion region. How does this connect to the neutral region?

In the neutral regions the majority carrier motion is dominated by drift (in contrast to the injected minority carrier current, which is determined by diffusion). Consider the $n$-type region. Here the bulk electron current that provides the junction current

$$
J_{n n}=-\mu_{n} n \frac{d E_{i}}{d x} .
$$

Since the two electron currents must be equal

$$
J_{n n}=J_{n p}
$$

it follows that

$$
\frac{d E_{F n}}{d x}=\frac{d E_{i}}{d x},
$$

i.e. the quasi-Fermi level follows the energy band variation. Thus, in a neutral region, the quasi-Fermi level for the majority carriers is the same as the Fermi level in equilibrium. At 
current densities small enough not to cause significant voltage drops in the neutral regions, the band diagram is flat, and hence the quasi-Fermi level is flat.

In the space charge region, $p n$ is constant, so the quasi-Fermi levels for holes and electrons must be parallel, i.e. both will remain constant at their respective majority carrier equilibrium levels in the neutral regions.

If an external bias $V$ is applied, the equilibrium Fermi levels are offset by $V$, so it follows that the quasi-Fermi levels are also offset by $V$,

$$
E_{F n}-E_{F p}=q_{e} V
$$

Consequently, the $p n$-product in non-equilibrium

$$
p n=n_{i}^{2} e^{\left(E_{F n}-E_{F p}\right) / k T}=n_{i}^{2} e^{q_{e} V / k T} .
$$

If the majority carrier concentration is much greater than the concentration due to minority carrier injection ("low-level injection"), the hole concentration at the edge of the $p$ region remains essentially at the equilibrium value. Consequentty, the enhanced $p n$-product increases the electron concentration.

$$
n_{p}(0)=n_{p 0} e^{q_{e} V / k T}
$$

Correspondingly, the hole concentration in the $n$-region at the edge of the depletion zone becomes

$$
p_{n}(0)=p_{n 0} e^{q_{e} V / k T}
$$

Since the equilibrium concentrations are

$$
\begin{aligned}
& n_{p 0}=\frac{n_{i}^{2}}{N_{a}} \\
& p_{n 0}=\frac{n_{i}^{2}}{N_{d}},
\end{aligned}
$$

the components of the diffusion current due to holes and electrons are

$$
\begin{gathered}
J_{n}=q_{e} D_{n} \frac{n_{i}^{2}}{N_{a} L_{n}}\left(e^{q_{e} V / k T}-1\right) \\
J_{p}=q_{e} D_{p} \frac{n_{i}^{2}}{N_{d} L_{p}}\left(e^{q_{e} V / k T}-1\right) .
\end{gathered}
$$

The total current is the sum of the electron and hole components

$$
J=J_{n}+J_{p}=J_{0}\left(e^{q_{e} V / k T}-1\right)
$$


where

$$
J_{0}=q_{e} n_{i}^{2}\left(\frac{D_{n}}{N_{a} L_{n}}+\frac{D_{p}}{N_{d} L_{p}}\right)
$$

This is the diode equation (or Shockley equation), which describes the current-voltage characteristic both under forward and reverse bias. Under forward bias $(V>0)$ the current increases exponentially. Under reverse bias $(V<0)$, the exponential term vanishes when he bias exceeds several $k T / q_{e}$ and the current becomes the reverse saturation current $J=-J_{0}$. For a uniform junction cross-section the current densities $J_{N}, J_{P}$, and $J_{0}$ can be replaced by their respective surrents.

Note that in the diode equation:

$\star$ 1. The bandgap does not appear explicitly (only implicitly in $J_{0}$ via $n_{i}$ ).

$\star 2$. The total current has two distinct components, due to electrons and holes.

$\star 3$. The electron and hole currents are generally not equal. The ratio:

$$
\frac{J_{n}}{J_{p}}=\frac{N_{d}}{N_{a}} \quad \text { if } \quad \frac{D_{n}}{L_{n}}=\frac{D_{p}}{L_{p}}
$$

$\star$ 4. Current flows for all values of $V$. However, when plotted on a linear scale, the exponential appears to have a knee, often referred to as the turn-on voltage.

$\star 5$. The magnitude of the turn-on voltage is determined by $J_{0}$. Diodes with different bandgaps will show the same behavior if $J_{0}$ is the same.

The discrepancies in the forward current between the measured results and the simple theory require the analysis of all processes in the depletion zone:

$\star$ 1. Generation-recombination in the depletion region (App. F)

$\star 2$. Diffusion current (as just calculated for the ideal diode).

$\star$ 3. High-injection region where the injected carrier concentration affects the potentials in the neutral regions.

$\star$ 4. Voltage drop due to bulk series resistance. 

Appendix C

Electrical Properties of Impurities and Defects 
To avoid confussion with exponential functions the symbol $q_{e}$ will be used for electronic charge instead of $e$. Although derivation of the diode equation in Appendix $E$ proceeded under the title "The forward-biased $p n$-junction", nothing in the assumptions and algebraic manipulations restricted the sign of the applied voltage. If a negative bias is applied to the junction, the minority carrier concentrations at the junction edges decrease with respect to thermal equilibrium and reverse the concentration gradient. Setting a reverse voltage $V \gg k T$ in the diode equation yields

$$
J=-J_{0}
$$

where

$$
J_{0}=q_{e} n_{i}^{2}\left(\frac{D_{n}}{N_{a} L_{n}}+\frac{D_{p}}{N_{d} L_{p}}\right) .
$$

In this ideal case the diode current at large reverse bias voltage would be determined by

* the doping concentrations $\left(N_{a}, N_{d}\right)$,

* the diffusion constants $\left(D_{n}, D_{p}\right)$, and

* the recombination lenghts $\left(L_{n}, L_{p}\right)$.

In reality, the measured currents are often orders of magnitude larger. Whereas the diode equation predicts the saturation of the reverse diode current at voltages greater than order $100 \mathrm{mV}(\sim 4 k T)$, one frequently observes a monotonically increasing current, which increases linearly with depletion width. This implies the presence of imperfections in the crystal that increase the reverse leakage current. For a uniform distribution of imperfections, the number of active sites will increase with the depletion volume.

\section{C.1 Emission and capture processes}

Figure C.1 (same figure as in Spieler 7.2) summarizes the emission and capture processes:

* Hole emission.

* Electron emission.

* Electron capture.

* Hole capture.

* Trapping. 
All of these processes are governed by Fermi statistics (or the Boltzmann approximation). Let us assume a concentration of centers $N_{t}$ whose energy level $E_{t}$ lies within the bandgap. The probability of a center being occupied is:

$$
f=\frac{1}{1+e^{\left(E_{t}-E_{F}\right) / k T}},
$$

and the concentration of vacant centers is:

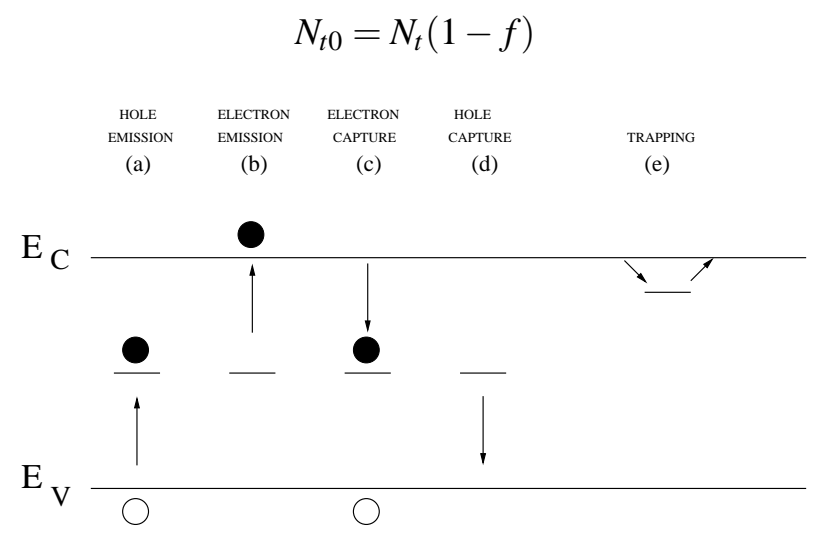

Figure C.1: Emission and capture processes through intermediate states. The arrows show the direction of electron transitions.

\section{C.1.1 Electron capture}

The rate of electron capture is proportional to the concentration of unoccupied centers

$$
\frac{d N_{n c}}{d t}=v_{t h} \sigma_{n} n N_{t 0}=v_{t h} \sigma_{n} n N_{t}(1-f),
$$

where $v_{t h}$ is the thermal velocity of an electron (about $10^{7} \mathrm{~cm} / \mathrm{s}$ at $300 \mathrm{~K}$ ), $\sigma_{n}$ is the capture cross-section, and $n$ is the concentration of electrons in the conduction band. The velocity enters because the capture centers are localized and an electron has to move near the center to be captured. The thermal velocity is superimposed on the much slower motion due to drift or diffusion, so the thermal velocity determines the number of defect sites scanned per unit time.

\section{C.1.2 Electron emission}

The rate of electron emission is proportional to the concentration of occupied centers $N_{n c}=$ $N_{t} f$. If the emission probability is $e_{n}$, the rate of electron emission

$$
\frac{d N_{n e}}{d t}=e_{n} N_{n c}=e_{n} N_{t} f
$$




\section{C.1.3 Hole capture}

Analogously to electrons, and since the hole capture corresponds to the transition of an electron from the center to the valence band, this process is proportional to the concentration of centers occupied by electrons $N_{t} f$.

$$
\frac{d N_{p c}}{d t}=v_{t h} \sigma_{p} p N_{t} f
$$

\section{C.1.4 Hole emission}

Proportional to the concentration of centers not occupied by electrons $N_{t}(1-f)$, so

$$
\frac{d N_{p e}}{d t}=e_{p} N_{t}(1-f)
$$

\section{C.1.5 Emission probabilities}

In equilibrium, the rates of the two processes that move electrons to and from the conduction band, capture and emission, must be equal. This seemingly trivial statement reflects the more profound principle in statistical mechanics of detailed balance, which states that under equilibrium conditions, every process and its reverse must proceed at exactly equal rates. Thus, the emission probability for electron and holes, respectively:

$$
\begin{aligned}
& \frac{d N_{n c}}{d t} \equiv \frac{d N_{n e}}{d t} \Rightarrow v_{t h} \sigma_{n} n N_{t}(1-f)=e_{n} N_{t} f \Rightarrow e_{n}=v_{t h} \sigma_{n} n \frac{1-f}{f} \\
& \frac{d N_{p c}}{d t} \equiv \frac{d N_{p e}}{d t} \Rightarrow v_{t h} \sigma_{p} p N_{t} f=e_{p} N_{t}(1-f) \Rightarrow e_{p}=v_{t h} \sigma_{p} p \frac{f}{1-f}
\end{aligned}
$$

The concentration of electrons in the conduction band is

$$
n=n_{i} e^{\left(E_{F}-E_{i}\right) / k T}=N_{c} e^{-\left(E_{c}-E_{F}\right) / k T}
$$

Using that $\frac{1-f}{f}=e^{\left(E_{t}-E_{F}\right) / k T}$, and $n=n_{i} e^{\left(E_{F}-E_{i}\right) / k T}=N_{c} e^{-\left(E_{c}-E_{F}\right) / k T}$, the emission probabilities for electrons and holes is, respectively:

$$
\begin{aligned}
& e_{n}=v_{t h} \sigma_{n} n_{i} e^{\left(E_{t}-E_{i}\right) / k T}=v_{t h} \sigma_{n} N_{c} e^{-\left(E_{c}-E_{i}\right) / k T} \\
& e_{p}=v_{t h} \sigma_{p} n_{i} e^{\left(E_{i}-E_{t}\right) / k T}=v_{t h} \sigma_{p} N_{v} e^{-\left(E_{t}-E_{v}\right) / k T}
\end{aligned}
$$

As intuitively expected, the emission probability grows exponentially as the energy level of the center approaches the band edge. 


\section{C.2 Recombination}

In detectors, recombination is responsible of loss of signal charge.

\section{C.2.1 Band-to-band recombination}

Incident radiation excites electrons from the valence to the conduction band, forming an electron-hole pair. The simplest recombination mechanism would be for electrons in the conduction band to recombine with holes in the valence band. The energy released in the recombination could be emitted as light or heat. Direct transitions from the conduction to the valence band in $\mathrm{Si}$ or Ge are extremely improbable and the recombination is made through intermediate states.

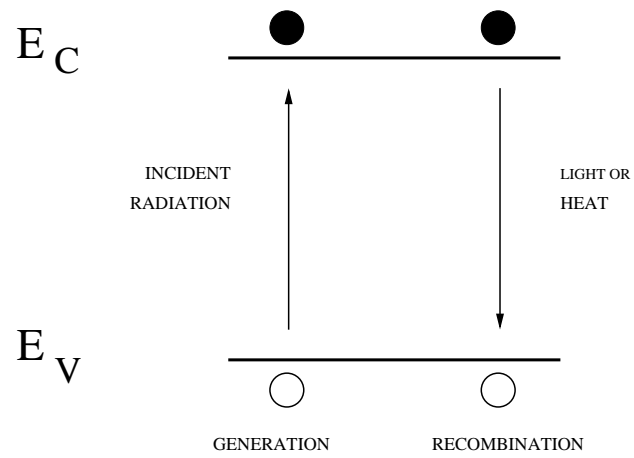

(a)

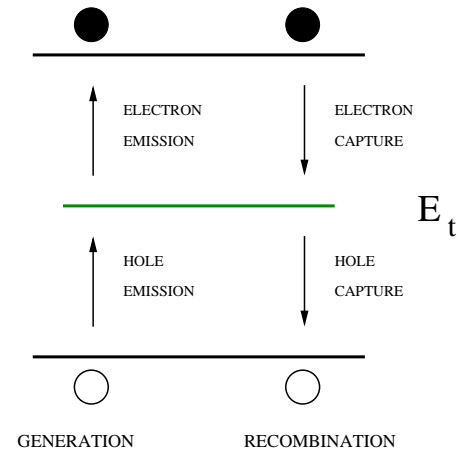

(b)

Figure C.2: Direct Transition (a), and Indirect Transition, via an intermediate state (b). The arrows show the direction of electron transitions. Direct transitions are extremely improbable in Silicon ("indirect bandgap"). In both cases it is supposed that the generation phase is due to an external irradiation and the bias voltage is zero (equilibrium, $\mathrm{V}$ bias $=0$ ).

\section{C.2.2 Recombination via intermediate states}

Consider a steady flux of radiation, for example light, leading to a uniform generation rate per unit volume $G_{L}$. To determine the effectiveness of centers as recombination sites, the charge due to the radiation will not be removed by an external circuit, but allowed to decay by recombination alone. In the steady state the rate at which electrons enter the conduction band (Fig. C.3) must equal the rate at which they leave it, the recombination rate $\frac{d N_{R}}{d t}$ :

$$
\frac{d n_{n}}{d t}=G_{L}-\frac{d N_{R}}{d t}=G_{L}-\left(\frac{d N_{n c}}{d t}-\frac{d N_{n e}}{d t}\right)=0 .
$$




$$
\frac{d n_{p}}{d t}=G_{L}-\frac{d N_{R}}{d t}=G_{L}-\left(\frac{d N_{p c}}{d t}-\frac{d N_{p e}}{d t}\right)=0 .
$$

Incident radiation takes the system out of thermal equilibrium, so none of the equilibrium carrier concentrations are valid, nor is the occupancy determined by the Fermi distribution. Instead, the concentrations $n$ and $p$ and the fractional occupancy $f$ depend on the radiation flux $G_{L}$. From the two expression above,

$$
\left(\frac{d N_{p c}}{d t}-\frac{d N_{p e}}{d t}\right)=\left(\frac{d N_{n c}}{d t}-\frac{d N_{n e}}{d t}\right)
$$

Replacing the rate expressions

$$
v_{t h} \sigma_{p} p N_{t} f-e_{p} N_{t}(1-f)=v_{t h} \sigma_{n} n N_{t}(1-f)-e_{n} N_{t}(1-f),
$$

and the emission probabilities, it is possible to extract the steady state fractional occupancy:

$$
f=\frac{\sigma_{n} n+\sigma_{p} n_{i} e^{\left(E_{i}-E_{t}\right) / k T}}{\sigma_{n}\left(n+n_{i} e^{\left(E_{t}-E_{i}\right) / k T}\right)+\sigma_{p}\left(p+n_{i} e^{\left(E_{i}-E_{t}\right) / k T}\right)} .
$$

This occupancy depends implicitly on the generation flux $G_{L}$, which determines $n$ and $p$. Electrons are continually captured and emitted by the center, and so are holes. If an electron and a hole recombine, this leads to a deficit in the emission rates of both electrons and holes. In the steady-state, the emission deficit for electrons and holes must be equal, so the net rate of recombination is the capture rate minus the emission rate, so replacing in its expression:

$$
\begin{gathered}
\frac{d N_{R}}{d t}=\left(\frac{d N_{n c}}{d t}-\frac{d N_{n e}}{d t}\right)=\left(\frac{d N_{p c}}{d t}-\frac{d N_{p e}}{d t}\right) \Rightarrow \\
\frac{d N_{R}}{d t}=\frac{\sigma_{p} \sigma_{n} v_{t h} N_{t}\left(p n-n_{i}^{2}\right)}{\sigma_{n}\left(n+n_{i} e^{\left(E_{t}-E_{i}\right) / k T}\right)+\sigma_{p}\left(p+n_{i} e^{\left(E_{i}-E_{t}\right) / k T}\right)}
\end{gathered}
$$

To simplify the equation and facilitate the interpretation of this result, let us assume that the capture cross-sections are equal: $\sigma_{n}=\sigma_{p}=\sigma$. Then

$$
\frac{d N_{R}}{d t}=\sigma v_{t h} N_{t} \frac{p n-n_{i}^{2}}{n+p+2 n_{i}\left(e^{\left(E_{t}-E_{i}\right) / k T}+e^{-\left(E_{t}-E_{i}\right) / k T}\right)} .
$$

From this expression one can see that the driving force of the recombination process is the excess carrier concentration $p n$ beyond the equilibrium concentration $n_{i}^{2}$. The third term in the denominator dscribes the relative occupancies of electrons and holes. A center close to the conduction band will have a higher occupancy of electrons than holes., so the recombination rate is limited by the hole population. Conversely, a center close to the valence band will have an excess of holes, so the population of electrons limits the recombination rate. The recombination rate is maximum when $E_{t}=E_{i}$, i.e. when the energy of the recombination center is at midgap. 
A special case of recombinaton is minority carrier injection. Let us consider holes injected into an $n$-type region, as in a forward biased diode. In this case $n_{n} \gg p_{n}$. Furthermore, since efficient recombination centers are far from the band edge, the Boltzmann approximation holds, so the equilibrium electron concentration

$$
n_{n} \gg n_{i} e^{\left(E_{t}-E_{i}\right) / k T} \text {. }
$$

Then the above expression for the recombination rate simplifies to

$$
\frac{d N_{R}}{d t}=\frac{\sigma_{n} \sigma_{p} v_{t h} N_{t}\left(n_{n} p_{n}-n_{i}^{2}\right)}{\sigma_{n} n_{n}}=\sigma_{p} v_{t h} N_{t}\left(p_{n}-p_{n 0}\right)=\frac{p_{n}-p_{n 0}}{\tau_{p}}
$$

where the carrier lifetime $\tau_{p}$ is

$$
\tau_{p}=\frac{1}{\sigma_{p} v_{t h} N_{t}}
$$

The lifetime of the holes in the $n$-bulk is independent of the concentration of the electrons. This is due to an abundance of electrons, so as soon as a hole is captured, an electron is available for immediate recombination. Hence, the hole concentration is the rate-limiting parameter. Conversely, if electrons are injected into $n$-type material where they are majority carriers, their lifetime will be significantly greater, since few holes are available for recombination. Minority carrier injection is the worst ase with respect to recombination, so "minority carrier lifetime" is a figure of merit used to characterize the presence of defects in semiconductors.

Recombination is important whenever the carrier concentration deviates from thermal equilibrium:

$$
p n>n_{i}^{2}
$$

This occurs

$\star$ in a forward-biased diode, or

$\star$ with incident radiation (and in equilibrium, at $\mathrm{V}=0$ bias)

\section{C.3 Carrier generation}

\section{C.3.1 Generation in the depletion region}

In a diode operated with reverse bias e.g. a radiation detector, with $V_{R} \gg \frac{k T}{q_{e}}$, all of the free carriers are swept from the depletion region (Fig. C.3). In this configuration only emission processes are important. 
Emission, in the absence of capture, can only proceed by alternating hole and electron emission, i.e. generation of electron-hole pairs. The rate of generation of electron-hole pairs can be determined from the previously derived expressions for the difference between capture and emission rates:

$$
\frac{d N_{R}}{d t}=\left(\frac{d N_{c}}{d t}-\frac{d N_{e}}{d t}\right)=\frac{\sigma_{p} \sigma_{n} v_{t h} N_{t}\left(p n-n_{i}^{2}\right)}{\sigma_{n}\left(n+n_{i} e^{\left(E_{t}-E_{i}\right) / k T}\right)+\sigma_{p}\left(p+n_{i} e^{\left(E_{i}-E_{t}\right) / k T}\right)}
$$

Since $d N_{c} / d t=\mathbf{0}$ and $p \ll n_{i}, n \ll n_{i}$,

$$
\frac{d N_{e}}{d t}=\frac{\sigma_{n} \sigma_{p} v_{t h} N_{t} n_{i}}{\sigma_{n} e^{\left(E_{t}-E_{i}\right) / k T}+\sigma_{p} e^{\left(E_{i}-E_{t}\right) / k T}}
$$

This often written as

$$
\frac{d N_{e}}{d t}=\frac{n_{i}}{2 \tau_{g}}
$$

where $\tau_{g}$ is called the generation lifetime. Considering again the case of equal crosssections $\sigma_{n}=\sigma_{p}=\sigma$, the generation rate becomes

$$
\frac{d N_{e}}{d t}=\frac{\sigma v_{t h} N_{t} n_{i}}{e^{\left(E_{i}-E_{t}\right) / k T}+e^{-\left(E_{i}-E_{t}\right) / k T}}
$$

which again shows that only states near the intrinsic Fermi level $E_{i}$, i.e. the mid-gap states, contribute significantly to the generation rate. Intuitively, this is easy to see in the "stepping stone" picture. Since the emission probabilities for electrons and holes increase exponentially with the separation from their respective band edges, the probability for sequential hole and electron emission is maximum at mid-gap.

The emission rate of carriers leads to an electrical current, the generation current, which increases with the density of centers. If the emission centers are distributed uniformly throughout the depletion width $\mathrm{W}$, the generation current density will be:

$$
J_{g e n}=q_{e} \frac{d N_{e}}{d t} W=q_{e} W \frac{\sigma v_{t h} N_{t} n_{i}}{e^{\left(E_{i}-E_{t}\right) / k T}+e^{-\left(E_{i}-E_{t}\right) / k T}} .
$$

\section{C.3.2 Generation in the neutral region}

In the neutral region the absence of a significant electric field means that any excess carriers due to generation move only by diffusion. Charges generated near the transition to the depletion region can reach the influence of the electric field and will be swept to the opposite electrode. This additional contribution to the reverse diode current is called the diffusion current. The starting point of the calculation is the steady state diffusion equation for minority carriers. Consider electrons generated in the $p$-region:

$$
D_{n} \frac{d^{2} n_{p}}{d x^{2}}-\frac{n_{p}-n_{p 0}}{\tau_{n}}=0
$$


Far from the space charge region the carrier concentration attains the thermal equilibrium value

$$
n_{P}(\infty)=n_{p 0}
$$

At the edge of the depletion region all carriers will be swept away by the electric field, so

$$
n_{P}(0)=0
$$

The solution to the diffusion equation for these boundary conditions is

$$
n_{P}(x)=n_{p 0}\left(1-e^{-x / L_{n}}\right)
$$

where

$$
L_{n} \sim \sqrt{D_{n} \tau_{n}}
$$

is the diffusion length of electrons in the $p$-region. This gives rise to an electrical current for those electrons in the $p$-region (and analogously, for holes in the $n$-region):

$$
\begin{gathered}
J_{d i f f, n}=-q_{e}\left(-\left.D_{n} \frac{d n_{p}}{d x}\right|_{x=0}\right)=q_{e} D_{n} \frac{n_{p 0}}{L_{n}}=q_{e} \frac{D_{n}}{L_{n}} \frac{n_{i}^{2}}{N_{a}} . \\
J_{d i f f, p}=-q_{e}\left(-\left.D_{p} \frac{d p_{n}}{d x}\right|_{x=0}\right)=q_{e} D_{p} \frac{p_{n 0}}{L_{p}}=q_{e} \frac{D_{p}}{L_{p}} \frac{n_{i}^{2}}{N_{d}} .
\end{gathered}
$$

The diffusion current increases with the square of the intrinsic carrier concentration, in contrast to the generation current in the depletion zone, which increases linearly witn $n_{i}$.

The generation rate in a neutral region depleted of minority carriers can be drastically different from the depletion region. for simplicity, let us assume that the diffusion lifetime is equal to the generation lifetime. Then the ratio of the two generation currents

$$
\frac{J_{\text {diff }, n}}{J_{\text {gen }}}=\frac{\frac{n_{p 0}}{\tau}}{\frac{n_{i}}{2 \tau} W}=2 \frac{n_{p 0}}{n_{i}} \frac{L_{n}}{W}=2 \frac{n_{i}}{N_{a}} \frac{L_{n}}{W}
$$

In an n-bulk Silicon radiation detector with a thin p-electrode, the diffusion length is limited by the electrod ethickness, i.e. $\sim 1 \mu \mathrm{m}$. For $n_{i} \approx 10^{10} \mathrm{~cm}^{-3}, N_{a} \approx 10^{15} \mathrm{~cm}^{-3}$, and W $\approx 300 \mu \mathrm{m}$ we have that the rate is:

$$
\frac{J_{\text {diff }, n}}{J_{\text {gen }}} \approx 3 \times 10^{-8}
$$

Thus concluding that in high-quality radiation detectors the generation current dominates. At higher temperatures the exponential increase in $n_{i}$ can increase the diffusion current so much that the generation current is negligible. 


\section{C.4 The origin of recombination and generation centers}

Recombination and generation centers can be introduced by

$\star$ impurity atoms,

$\star$ structural imperfections,

$\star$ radiation damage (displacement of atoms from lattice sites).

All three defect mechanisms can create states distributed throughout the bandgap, since only mid-gap states can contribute significantly to generation and recombination, in a continuum of states statistics automatically select the states near mid-gap.

\section{C.5 The diode equation revisited}

\section{C.5.1 Reverse current}

Both the generation and diffusion currents invariably override the ideal reverse saturation current

$$
J_{0}=q_{e} n_{i}^{2}\left(\frac{D_{n}}{N_{a} L_{n}}+\frac{D_{p}}{N_{d} L_{p}}\right) \ll J_{d i f f}+J_{g e n},
$$

so the diode equation becomes:

$$
J=J_{R}\left(e^{q_{e} V / k T}-1\right) .
$$

The reverse current $J_{R}$ for voltages $>\mathbf{3 k T} / q_{e}$ is the sum of the diffusion and generation currents:

$$
J_{R}=q_{e} n_{i}^{2}\left(\frac{1}{N_{a}} \sqrt{\frac{D_{n}}{\tau_{n}}}+\frac{1}{N_{d}} \sqrt{\frac{D_{p}}{\tau_{p}}}\right)+q_{e} \frac{n_{i}}{2 \tau_{g}} W .
$$

Whether the generation or diffusion current dominates can be determined from the temperature coefficient. The diffusion current scales with $n_{i}^{2}$, so

$$
\frac{d J_{R}}{d T}=J_{R} \frac{E_{g}}{k T^{2}}
$$

whereas the generation current scales with $n_{i}$, yielding

$$
\frac{d J_{R}}{d T}=J_{R} \frac{E_{g}}{2 k T^{2}} .
$$


In practice, a plot of $\log J_{R} v s .1 / k T$ will yield a slope of $-E_{g}$ for diffusion and approximately $-E_{g} / 2$ for generation dominated operation. At sufficiently high temperature, diffusion will always dominate.

In radiation damaged diodes the generation current dominates even after rather low fluences, so the reverse bias current

$$
I_{R}(T) \propto v_{t h} n_{i} \propto v_{t h} \sqrt{N_{c} N_{\nu}} e^{-E / 2 k T} \propto T^{2} e^{-E / 2 k T},
$$

where $E \approx E_{g}$, depending on the impurity or defect energy level. The ratio of currents at two temperatures $T_{1}$ and $T_{2}$ is

$$
\frac{I_{R}\left(T_{2}\right)}{I_{R}\left(T_{1}\right)}=\left(\frac{T_{2}}{T_{1}}\right)^{2} \exp \left[-\frac{E}{2 k}\left(\frac{T_{1}-T_{2}}{T_{1} T_{2}}\right)\right] .
$$

Cooling to $0^{\circ} \mathrm{C}$ typically reduces the reverse bias current to $1 / 6$ of its value at room temperature. Since after irradiation the leakage current initially decreases with time, pronounced short term and long term annealing components are observed and precise fits to the annealing curve require a sum of exponentials. In practice, the variation of leakage current with temperature is very reproducible from device to device, even after substantial doping changes due to radiation damage. The leakage current can be used for dosimetry and diodes are offered commercially specifically for this purpose (transistors being used to measure temperature).

\section{C.5.2 Forward current}

Recombination in the depletion region also affects the forward diode characteristic. Experimental results can generally be described by introducing an "ideality factor" $n$

$$
J=J_{R}\left(e^{q_{e} V / n k T}-1\right),
$$

where

$* n=2$ when the recombination current dominates

$* n=1$ when the current is dominated by diffusion in the neutral regions

In practical diode, $n$ lies between 1 and 2.

At very low currents the generation currents dominate. Since these currents are opposite to the forward injection current, one observes a change of sign in the current flow at low voltage. 


\section{C.5.3 Comments}

In radiation detectors the reverse current is of primary interest, as it is a source of shot noise. Nevertheless, the forward current-voltage characteristic can provide useful diagnostic information. Since recombination and generation are both maximized for mid-gap states, one commonly observes that devices with large generation currents also exhibit high recombination rates.

This has promoted a tendency to characterize both phenomena by one parameter, the minority carrier lifetime. However, generation and recombination are two distinct phenomena. First, their temperature dependencies differ, and second, it is not at all assured that a state is equally effective at generation as it is at recombination. 
CARRIER GENERATION PROBABILITIES

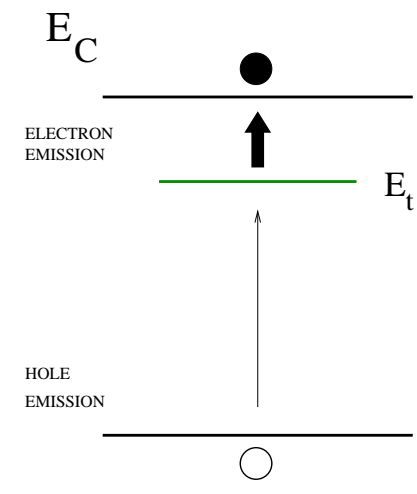

$\mathrm{E}_{\mathrm{V}}$

(a)

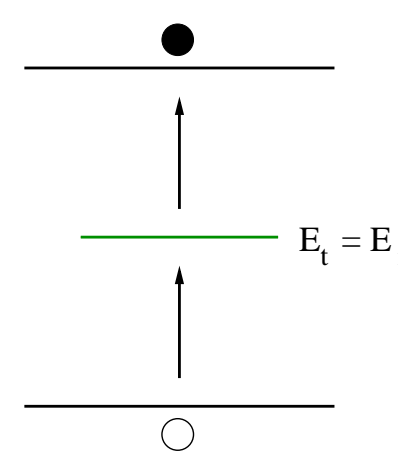

(b)

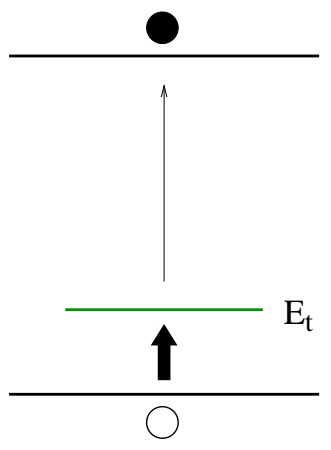

(c)

CARRIER RECOMBINATION PROBABILITIES

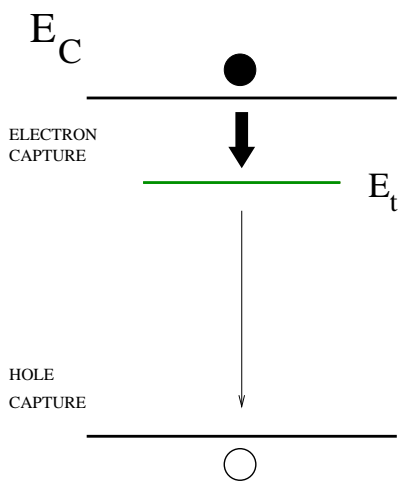

$\mathrm{E}_{\mathrm{V}}$

(a)

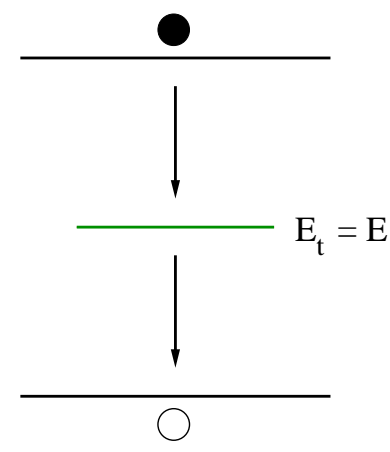

(b)

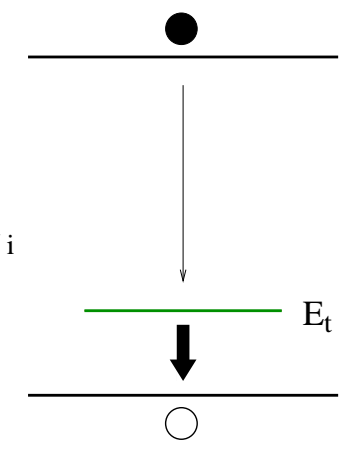

(c)

Figure C.3: Carrier generation and recombination probabilities. The width of the arrows gives an idea of the probability of each transition. The mid-gap case (the defect energy is that of the intrinsic semiconductor) is preferred for a sequential double transition. For the generation figure (up) it is supposed a reverse bias $(\mathrm{V}<0)$ in order to swept the carriers away, and not considering their recombinations. For the recombination figure (down) the generation phase is supposed to be created by a forward bias $(\mathrm{V}>0)$ ( or an external radiation with bias $\mathrm{V}=0$ ). 

Appendix D

Type Inversion and Doping Profile 

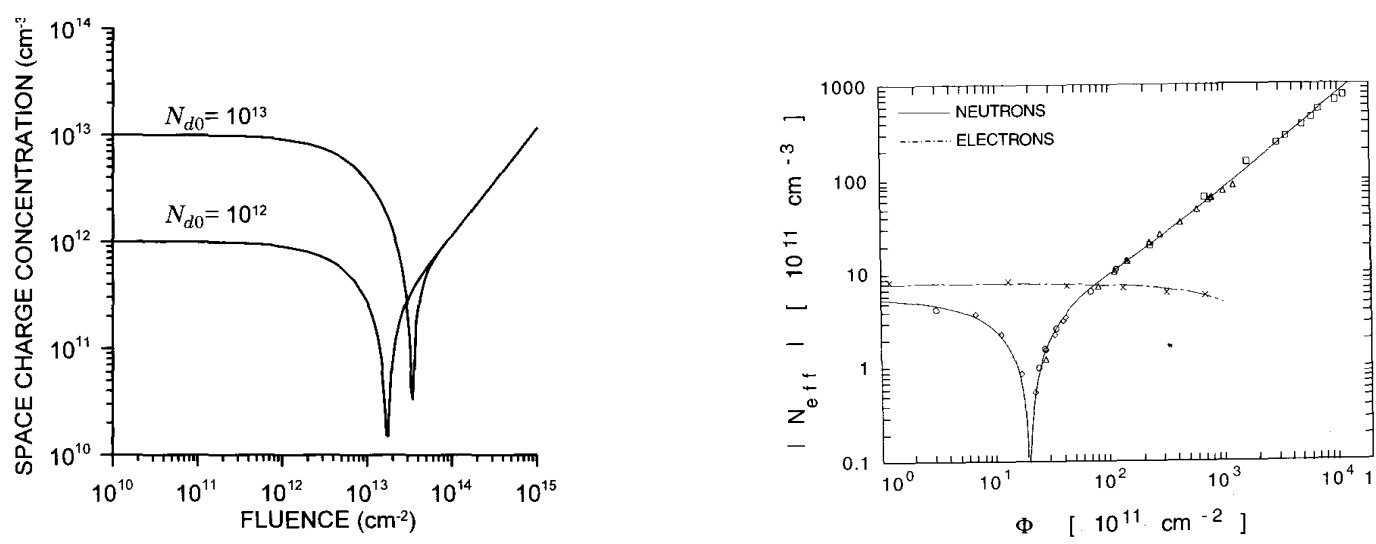

Figure D.1: Left: calculated space charge vs. high-energy proton fluence for silicon with initial donor concentrations $N_{d 0}$ of $10^{12}$ and $10^{13} \mathrm{~cm}^{-3}$. Whith an infinitely fine calculation grid both distributions would dip to zero (Spieler, 2005). Right: Fluence dependence of the magnitude of the effective doping for an $n$-type silicon wafer irradiated with $1 \mathrm{MeV}$ neutrons equivalent. The data have been corrected for self-annealing occurring already during the extended irradiation period. Also shown is the much smaller effect of irradiation with $1.8 \mathrm{MeV}$ electrons also scaled to $1 \mathrm{MeV}$ neutron equivalent NIEL (Lutz, 1999).

\section{D.1 Double junction models}




\section{Distribution of vacancies created by a $50 \mathrm{keV}$ Si-ion in silicon (typical recoil energy for $1 \mathrm{MeV}$ neutrons)}
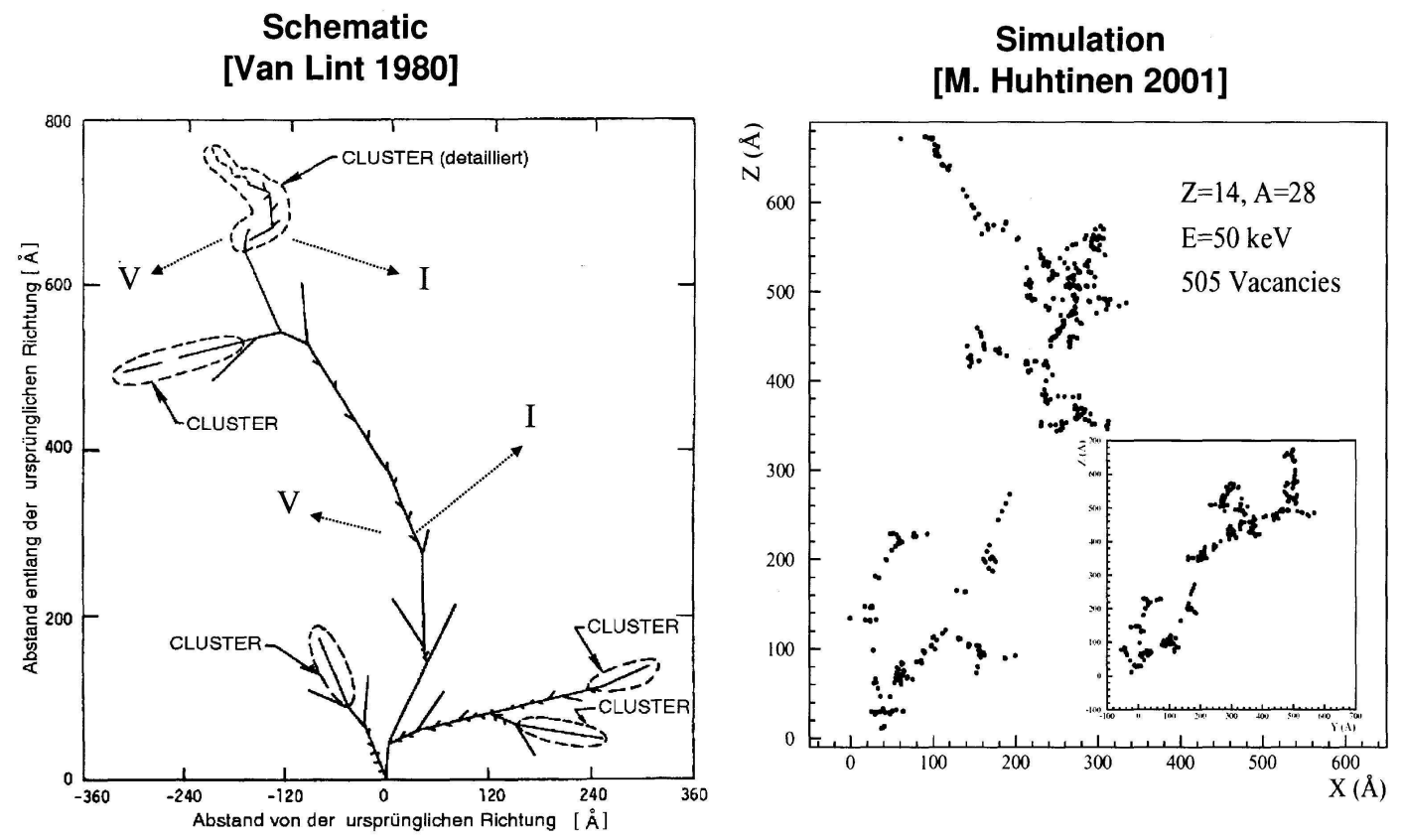

Figure D.2: Model and simulation of clustered defects.

A large number of measurements suggesting that large electric fields exist on both sides of an irradiated silicon diode has given rise to several attempts to model the effect $[83,81]$. The most recent of these studies by Eremin, Verbitskaya and Li (EVL) [81] is based upon a modification of the Shockley-Read-Hall (SRH) statistics. The EVL model produces an effective space charge density $\rho_{e f f}$ from the trapping of leakage current by one acceptor trap and one donor trap. The effective charge density is related to the occupancies and densities of traps:

$$
\rho_{e f f}=e\left[N_{D} f_{D}-N_{A} f_{A}\right]+\rho_{\text {dopants }}
$$

where $N_{D}$ and $N_{A}$ are the densities of donor and acceptor trapping states, respectively; $f_{D}$ and $f_{A}$ are the occupied fractions of the donor and acceptor states, respectively; and $\rho_{\text {dopants }}$ is the charge density due to ionized dopants. Charge flows to and from the trapping states due to generation and recombination (Appendix $\mathrm{C}$ ). The occupied fractions are given by the following standard SRH expressions: 


$$
\begin{gathered}
f_{D}=\frac{v_{h} \sigma_{h}^{D} p+v_{e} \sigma_{e}^{D} n_{i} e^{E_{D} / k T}}{v_{e} \sigma_{e}^{D}\left(n+n_{i} e^{E_{D} / k T}\right)+v_{h} \sigma_{h}^{D}\left(p+n_{i} e^{-E_{D} / k T}\right)} \\
f_{A}=\frac{v_{e} \sigma_{e}^{A} n+v_{h} \sigma_{h}^{A} n_{i} e^{-E_{A} / k T}}{v_{e} \sigma_{e}^{A}\left(n+n_{i} e^{E_{A} / k T}\right)+v_{h} \sigma_{h}^{A}\left(p+n_{i} e^{-E_{A} / k T}\right)}
\end{gathered}
$$

where $v_{e}$ and $v_{h}$ are the thermal speeds of electrons and holes; $\sigma_{e}^{D}$ and $\sigma_{h}^{D}$ are the electron and hole capture cross sections for the donor trap; $\sigma_{e}^{A}$ and $\sigma_{h}^{A}$ are the electron and hole capture cross sections for the acceptor trap; $n$ and $p$ are the densities of free electrons and holes; $n_{i}$ is the intrinsic density of carriers; $E_{D}$ and $E_{A}$ are the activation energies relative to the mid-gap energy of the donor and acceptor states, respectively. The generationrecombination current caused by the SRH statistics for single donor and acceptor states is given by the following expression:

$$
U=\frac{v_{h} v_{e} \sigma_{h}^{D} \sigma_{e}^{D} N_{D}\left(n p-n_{i}^{2}\right)}{v_{e} \sigma_{e}^{D}\left(n+n_{i} e^{E_{D} / k T}\right)+v_{h} \sigma_{h}^{D}\left(p+n_{i} e^{-E_{D} / k T}\right)}+\frac{v_{h} v_{e} \sigma_{h}^{A} \sigma_{e}^{A} N_{A}\left(n p-n_{i}^{2}\right)}{v_{e} \sigma_{e}^{A}\left(n+n_{i} e^{E_{A} / k T}\right)+v_{h} \sigma_{h}^{A}\left(p+n_{i} e^{-E_{A} / k T}\right)}
$$

Within the EVL model, the four trapping cross sections are fixed to $10^{-15} \mathrm{~cm}^{2}$. The leakage current is generated from an additional SRH trapping state that is introduced for this purpose but is assumed not to trap any charge. The donor and acceptor states are assumed not to generate leakage current which, given the smal size of the cross sections, is a self-consistent assumption. The densities of the donor and acceptor states, $N_{D}$ and $N_{A}$ are tuned to the Transient Charge Technique (TCT) data. The parameters of the model are given in Table D.1. The trap densities are scaled to fluence and are given in terms of introduction rates $g_{i n}=N_{A / D} / \Phi_{e q}$.

Table D.1: Parameters of the EVL Model [81].

\begin{tabular}{|lllll|}
\hline \multicolumn{5}{|c|}{ EVL Model Parameters } \\
\hline \hline Trap & $\mathrm{E}(\mathrm{eV})$ & $\mathrm{g}_{\text {int }}\left(\mathrm{cm}^{-1}\right)$ & $\sigma_{e}\left(\mathrm{~cm}^{2}\right)$ & $\sigma_{h}\left(\mathrm{~cm}^{2}\right)$ \\
\hline Donor & $\mathrm{E}_{V}+0.48$ & 6.0 & $1 \times 10^{-15}$ & $1 \times 10^{-15}$ \\
Acceptor & $\mathrm{E}_{C^{-}} 0.52$ & 3.7 & $1 \times 10^{-15}$ & $1 \times 10^{-15}$ \\
\hline
\end{tabular}

To illustrate the EVL model a figure from Ref. [83] has been reproduced, shown in Fig. D.3. Figure D.3(a) shows an uniform current density flowing across a reverse-biased junction. Since holes are produced uniformly across the junction and flow to the p+ backplane, the hole current density increases linearly with increasing $z$ from the $n+$ implant to the $p+$ implant. The electrons flow to the $n+$ implant and the electron current density increases 


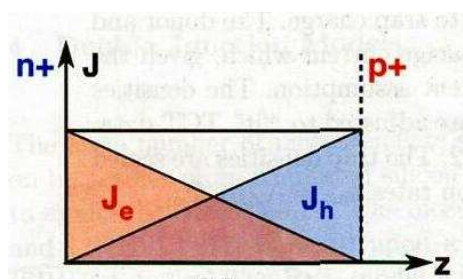

(a)

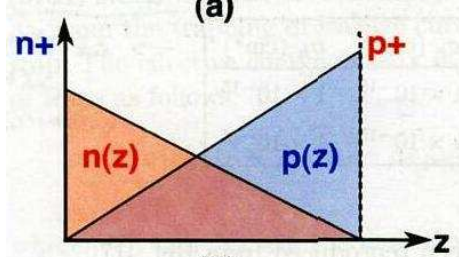

(b)

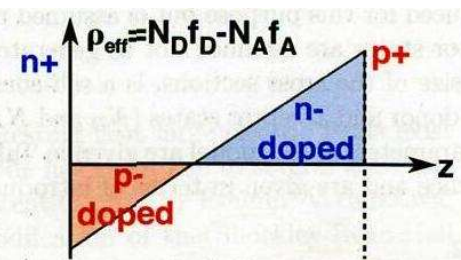

(c)

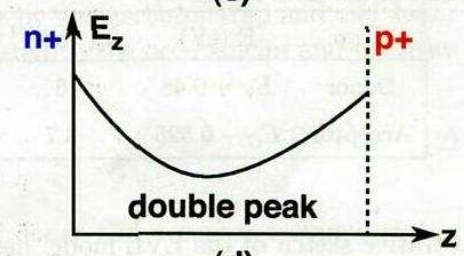

(d)

Figure D.3: EVL model.

with decreasing $z$. The actual carrier densities depend upon the details of the fields and mobilities but vary monotonically across the junctions as shown in Fig. D.3(b). The trapping of the mobile carriers produces a net positive space charge density near the p+ backplane and a net negative space charge density near the $n+$ implant as shown in Fig. D.3(c). Since positive space charge corresponds to $n$-type doping and negative space charge corresponds to p-type doping, there are p-n junctions at both sides of the detector. The electric field in the sensor follows from a simultaneous solution of Poisson's equation and the carrier continuity equations. The resulting z-component of the electric field is shown in Fig. D.3(d). It varies with approximately quadratic dependence upon $z$ having a minimum at the zero of the space charge density and two maxima in the borders at both implants [83].

\section{D.2 Junction models and experimental data}

In order to compare the experimental results with the introduced double junction model, let us use data from sensor L00-f843 as a working example ${ }^{1}$. In its depletion voltage historical evolution plot (Fig. D.4) it is possible to select three depletion voltage points $\left(V_{d e p, i}=V_{d e p}\left(L_{i}\right)\right.$, with $i=3$ ) corresponding to the three significative luminosities of each one of the three states that the sensor has gone through:

- $L_{1}=499 \mathrm{pb}^{-1}$, before inversion (sate 1)

- $L_{2}=2041 \mathrm{pb}^{-1}$, inversion point (minimum of the fitting curve $V_{d e p}(L)$, state 2)

\footnotetext{
${ }^{1}$ Altough the double junction model is intended to be appliable to a double-sided sensor -not the case of L00- we can assume that a phi side sensor of SVX would behave in a similar way than a typical L00.
} 

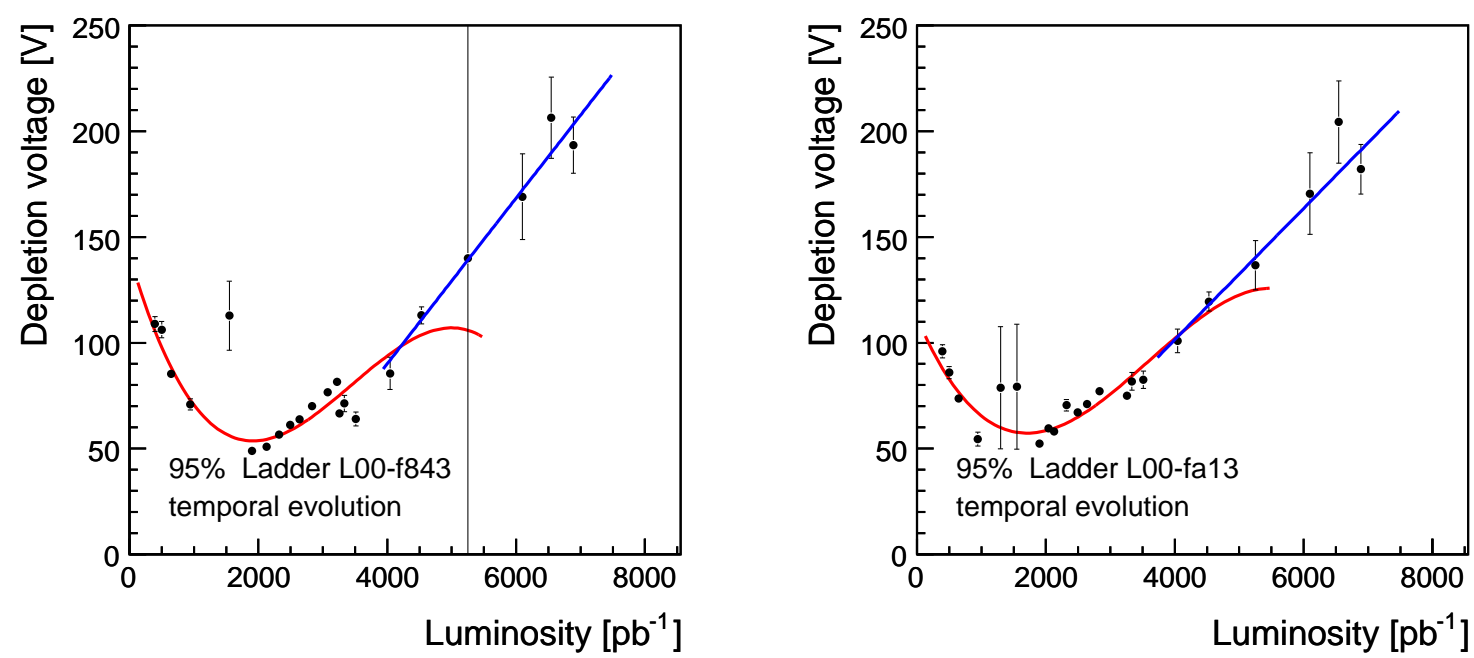

Figure D.4: Type inversion experimental data. Example of two L00 opposite sensors: f843 and fa13.

- $L_{3}=6888 p b^{-1}$, after inversion (sate 3)

$$
\begin{gathered}
V_{d e p, 1}=V_{d e p}(499)=115 V \\
V_{d e p, 2}=V_{d e p}(2041)=50 V \\
V_{d e p, 3}=V_{d e p}(6888)=195 V
\end{gathered}
$$

Since the change in space charge appears as a change in doping level, the net space charge is commonly referred to as an effective doping level $N_{e f f}$. In the state after inversion the detector functions as before and no change in bias polarity is needed, but to transport charge through the full detector thickness $d$ the voltage must be raised proportionally to the increase in space charge ${ }^{2}$. In analogy to conventional diode operation this is often referred to as the depletion voltage $V_{d e p}=\frac{e}{2 \varepsilon}\left|N_{e f f}\right| d^{2}$. Using this expression with Eq. D.7 it is possible to work out the numerical values of $N_{e f f, i}$ :

$$
V_{d e p, 1}=V_{d e p}(499)=115 V=\frac{e}{2 \varepsilon}\left|N_{e f f, 1}\right| d^{2} \Rightarrow\left|N_{e f f, 1}\right|=\frac{2 \varepsilon}{e d^{2}} V_{d e p, 1}
$$

\footnotetext{
${ }^{2}$ The depletion width $w_{d}$ is considered here to be constant along luminosity, this is, the sensor is supposed to be fully depleted during inversion.
} 


$$
\begin{gathered}
V_{d e p, 2}=V_{d e p}(2041)=50 \mathrm{~V}=\frac{e}{2 \varepsilon}\left|N_{e f f, 2}\right| d^{2} \Rightarrow\left|N_{e f f, 2}\right|=\frac{2 \varepsilon}{e d^{2}} V_{d e p, 2} \\
V_{d e p, 3}=V_{d e p}(6888)=195 \mathrm{~V}=\frac{e}{2 \varepsilon}\left|N_{e f f, 3}\right| d^{2} \Rightarrow\left|N_{e f f, 3}\right|=\frac{2 \varepsilon}{e d^{2}} V_{d e p, 3}
\end{gathered}
$$

Where $\left|N_{e f f, i}\right|$ are known constants ${ }^{3}$.

The assumption of $\mathbf{N}_{e f f}$ to be a single number (i.e the sum of positive and negative charge centers in the space charge of the depleted sensor; or $\mathbf{N}_{e f f, i}$ if considering the charge at a particular luminosity $i$ ) is a useful simplification, but does not considers the deeper structure or distribution of the charge in the depleted zone. A generalization hence may be useful and so $\mathbf{N}_{e f f}$ could be redefined as a distribution along the $\mathbf{z}$-axis of the sensor. The sensor's $\mathbf{z}$ axis is perpendicular to the plates and positive upwards, from $\mathbf{n}+$ contact to $\mathbf{p}+$ contact or, similarly, along the direction of movement of particles coming from the collision point and traversing the sensor. After this reasoning we define the functions $\mathbf{N}_{e f f}(\mathbf{z})$ and $\mathbf{N}_{e f f}(\mathbf{z}, \mathbf{L})$.

In Table D.3 are shown two possibilities for the space charge distribution of an irradiated sensor: an homogeneous distribution and an inhomogeneous one. Its first row is dedicated to the homogeneous case. The space charge originally positive has evolved to a situation where new negative charges have been created. The number of this negative charges being superior to the number of original positive charges (type-inversion) makes a global addition of $\mathbf{N}_{e f f}=(5-)$. However, the distribution of those negative centers is homogeneous inside the sensor's bulk. In the picture this is representated as partial row-by-row computations of the $\mathbf{N}_{e f f}$ (an illustrative and simple discretization of $\mathbf{N}_{e f f}(\mathbf{z})$ ). As a result of homogenity, those partial computations have always constant values for each $r$-esim row: $\mathbf{N}_{e f f, r}=(1-)$. The plot of $\mathbf{N}_{e f f}(\mathbf{z})$ for the homogeneous case shows a p-like region (net negative charge) and constant along the $z$-axis, from $p+$ contact to $n+$ contact. Since the $p+$ contact and the $p$ radiation-generated region share the same doping class, together they form the p-part of the global pn-junction, where the n-part is obviously the $n+$ contact. The formation of this new pn-junction after irradiation is responsible of the shift of the depletion voltage (Fig. D.2) and the fact that the polarity of bias voltage does not need to be reversed after type-inversion.

The second row of Table D.3 shows a model of inhomogeneous distribution of charge in the sensor's space charge. The plot of $\mathbf{N}_{e f f}(\mathbf{z})$ shows that negative centers are located close to the $n+$ contact while positive centers are on the $p+$ contact, a situation that creates a

$$
\begin{array}{r}
{ }^{3}\left|N_{e f f, i}\right|=\left(1.45 \cdot 10^{10} \mathrm{~cm}^{-3} \mathrm{~V}^{-1}\right) \cdot\left(V_{\text {dep }, i}\right), \text { then, } \\
\qquad\left|N_{e f f, 1}\right|=1.66 \cdot 10^{12} \mathrm{~cm}^{-3} \\
\left|N_{e f f, 2}\right|=7.25 \cdot 10^{11} \mathrm{~cm}^{-3} \\
\left|N_{e f f, 3}\right|=2.82 \cdot 10^{12} \mathrm{~cm}^{-3}
\end{array}
$$


double pn-junction. A double pn-junction is an arrange also able to transfer the depletion zone without necessity of inverting bias polarity.

It is remarkable that this inhomogeneous model is compatible with the homogeneous one. In effect, altough the partial computations output different values, the global account of the space charge centers equals (5-), the same number as the homogeneous case. In a more correct formulation we can write that the area $A$ under both functions is the same:

$$
\begin{aligned}
A\left[N_{\text {eff }, \text { homo }}\right] & =A\left[N_{\text {eff }, \text { inhomo }}\right] \\
\int_{z=0}^{z=d} N_{\text {eff }, \text { homo }}(z) d z & =\int_{z=0}^{z=d} N_{\text {eff, inhomo }}(z) d z .
\end{aligned}
$$

With this idea we can expand Eq. D.18:

$$
\begin{gathered}
V_{d e p, 1}=V_{d e p}(499)=115 V=\frac{e}{2 \varepsilon}\left|N_{e f f, 1}\right| d^{2} \Rightarrow\left|N_{e f f, 1}\right|=\frac{2 \varepsilon}{e d^{2}} V_{d e p, 1}=\int_{0}^{d} N_{e f f, 1}(z) d z \\
V_{d e p, 2}=V_{d e p}(2041)=50 V=\frac{e}{2 \varepsilon}\left|N_{e f f, 2}\right| d^{2} \Rightarrow\left|N_{e f f, 2}\right|=\frac{2 \varepsilon}{e d^{2}} V_{d e p, 2}=\int_{0}^{d} N_{e f f, 2}(z) d z \text { (D.17) } \\
V_{d e p, 3}=V_{d e p}(6888)=195 \mathrm{~V}=\frac{e}{2 \varepsilon}\left|N_{e f f, 3}\right| d^{2} \Rightarrow\left|N_{e f f, 3}\right|=\frac{2 \varepsilon}{e d^{2}} V_{d e p, 3}=\int_{0}^{d} N_{e f f, 3}(z) d z
\end{gathered}
$$

Now the situation has been transformed into a variational calculus problem. We have to find distributions with a boundary condition on its area $A$ :

$$
\begin{aligned}
& N_{\text {eff,1 }}(z) \text { such that : } A\left[N_{e f f, 1}(z)\right]=c t_{1} ; \text { this is : } \int_{0}^{d} N_{e f f, 1}(z) d z=\left|N_{e f f, 1}\right| \quad \sqrt{ } \\
& N_{\text {eff, } 2}(z) \quad \text { such that }: \quad A\left[N_{\text {eff, } 2}(z)\right]=c t_{2} ; \quad \text { this is }: \quad \int_{0}^{d} N_{e f f, 2}(z) d z=\left|N_{\text {eff, }, 2}\right| \\
& N_{\text {eff, } 3}(z) \text { such that : } \quad A\left[N_{e f f, 3}(z)\right]=c t_{3} ; \text { this is : } \int_{0}^{d} N_{e f f, 3}(z) d z=\left|N_{\text {eff }, 3}\right|
\end{aligned}
$$

Let us remember that $N_{e f f, 1}(\mathrm{z})$ is the state-1 (before irradiation) space charge distribution. This original distribution is known: a positive constant equaling the initial n-type doping of the bulk. Instead, $N_{e f f, 2}(z)$ and $N_{e f f, 3}(z)$ (inversion-point distribution and afterirradiation distribution, respectively) are unknown.

With respect to $N_{e f f, 2}(\mathrm{z})$ we can affirm that it will be a distribution with the same number of positive and negative centers (perhaps plus a 'shift' constant, maybe related with bias current loses around the electric circuit of the sensor, as shown in Figure D.6). 
Distribution $N_{e f f, 3}(\mathbf{z})$ will have larger number of negative centers than positive ones (and eventually would also be affected by the 'shift' constant), as it is possible to infer from the definition (and observed fact in the inversion curves) of type-inversion.

The complete computation of the solution may be complicated since many distributions can match the boundary conditions. However, there is a simple way to model the space charge distributions if we make a simplification (and, unfortunately, a reduction of the possibilities for the satisfying functions): instead of considering the space charge distribution as linear along z-axis (Eremin et. al) [81] we may model it with a cosine function ${ }^{4}$ $y(z)=-\cos (z)-C_{1}$, being $C_{1}$ a positive constant (Fig. D.5). This negative cosine function satisfies the boundary conditions of negative space charge close to $n+$ contact and positive space charge close to $p+$ contact (assymetry). A cosine modelization of the space charge yields to a doble pn-junction, confirming $y(z)=-\cos (z)-C_{1}$ as an able solution ${ }^{5}$ of the problem.

The electric field inside the sensor's bulk is computed upon:

$$
\nabla E(z)=-\frac{e}{\varepsilon \varepsilon_{0}} N_{e f f}(z)
$$

Then, $\mathrm{E}(\mathrm{z})$ is parabolic for the linear case and sinusoidal for the trigonometric case.

\footnotetext{
${ }^{4}$ This trigonometric approximation is valid for both distributions $N_{e f f, 2}(\mathrm{z})$ (setting $C_{1}=0$ ), and $N_{e f f, 3}(\mathrm{z})$ (setting $\left.C_{1} \neq 0\right)$.

${ }^{5}$ And of course the family $y(z)=-A \cos \left(n z-C_{0}\right)-C_{1}$ (where $n$ is required to be odd in order to satisfy the boundary condition of an assymetrc distribution), that mathematically yields to a situation of $n$-junctions. The experimental verification of this possibility is out of the scope of this work. An hypothetical related mechanism may be the fact that the clusters, being a source of vacants, could trigger the creation of E-centers close to the $\mathrm{n}+$ contact side so creating a negative space charge seeding region.
} 
Table D.2: Type-inversion evolution models.

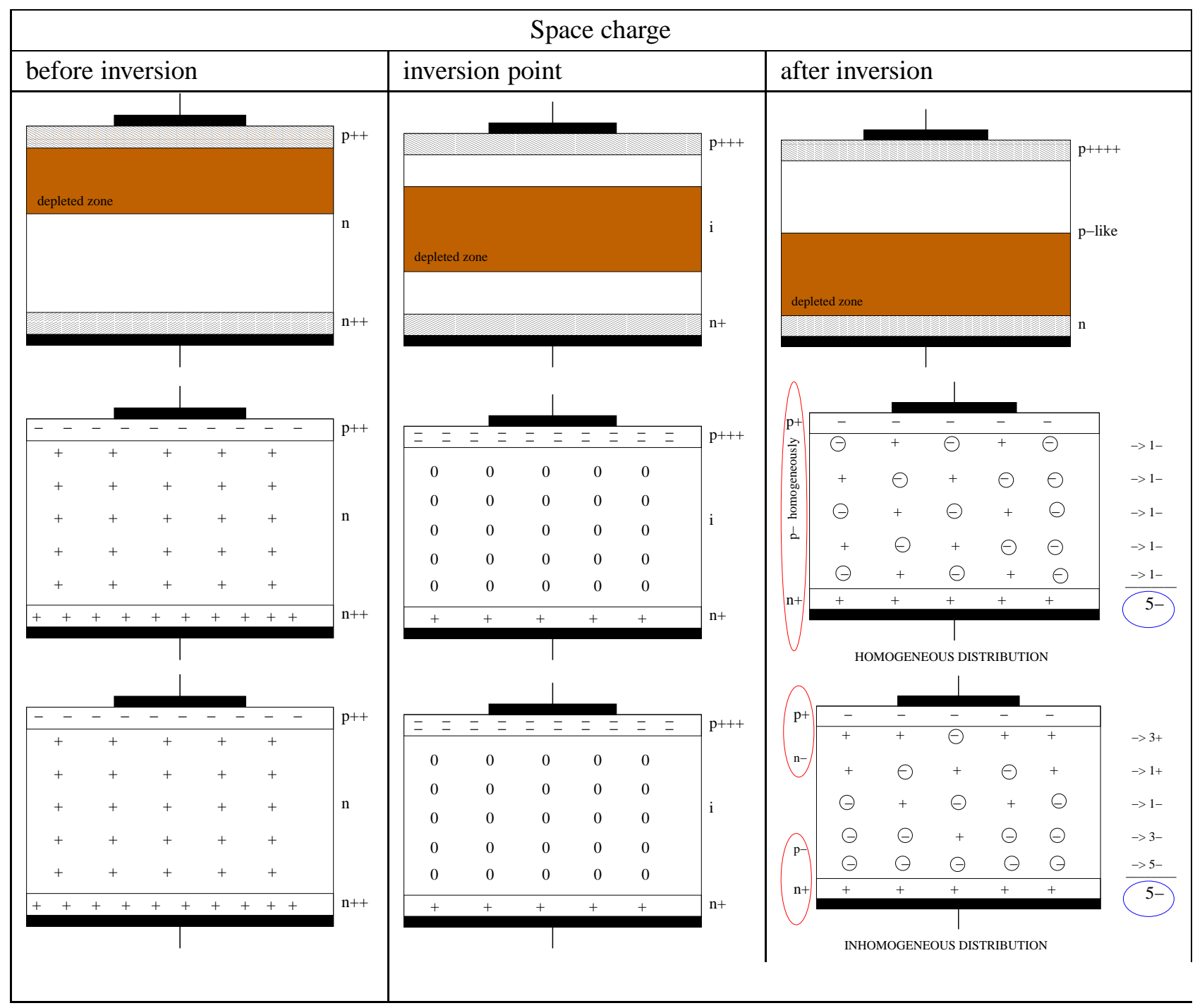


Table D.3: Homogeneous and inhomogeneously distributed space charge models of an irradiated sensor.

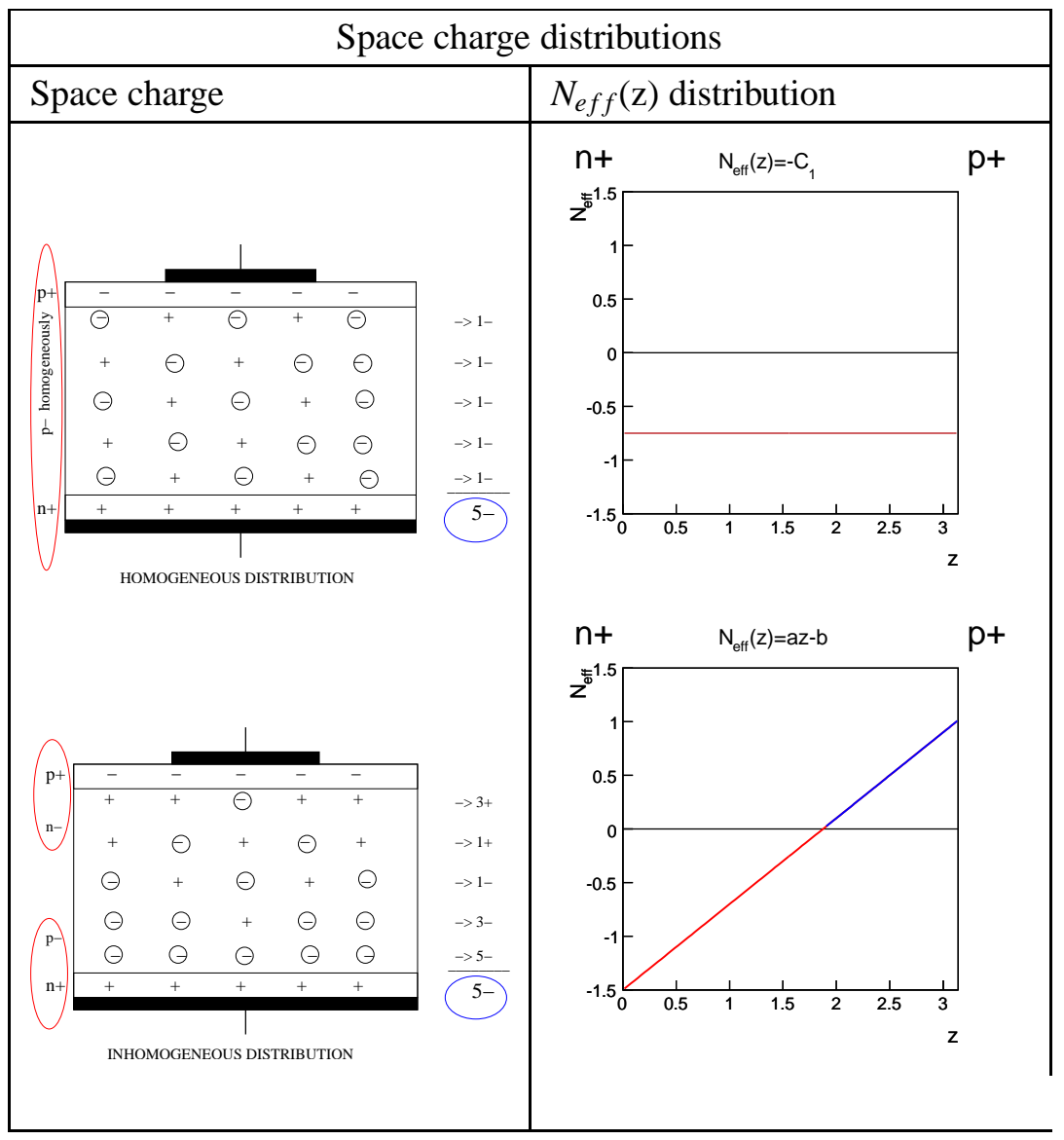




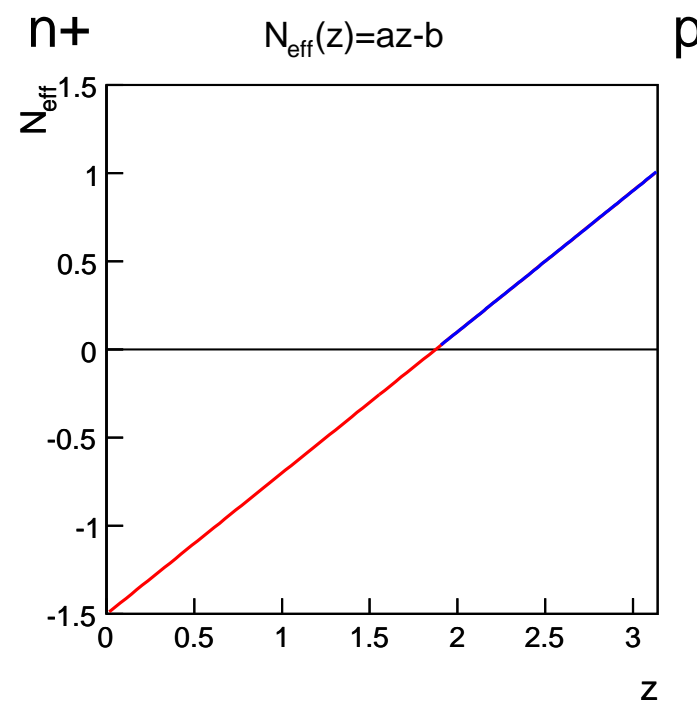

$p+$

$\mathrm{n}+$

$N_{\text {eff }}(z)=-\cos (z)-C_{1}$ $\mathrm{p}+$ $z^{\frac{5}{0}}$ $z^{\frac{5}{D^{1}}}$

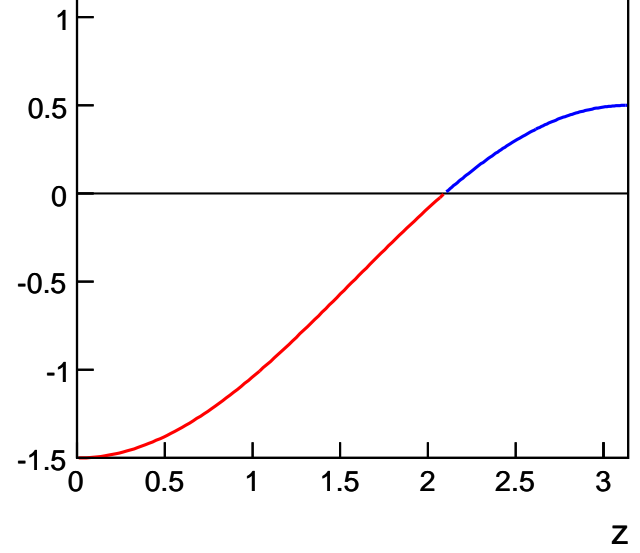

Figure D.5: Inhomogeneous Model (double pn-junction). The linear distribution of the original inhomogeneous model could be replaced by a trigonometric distribution. 


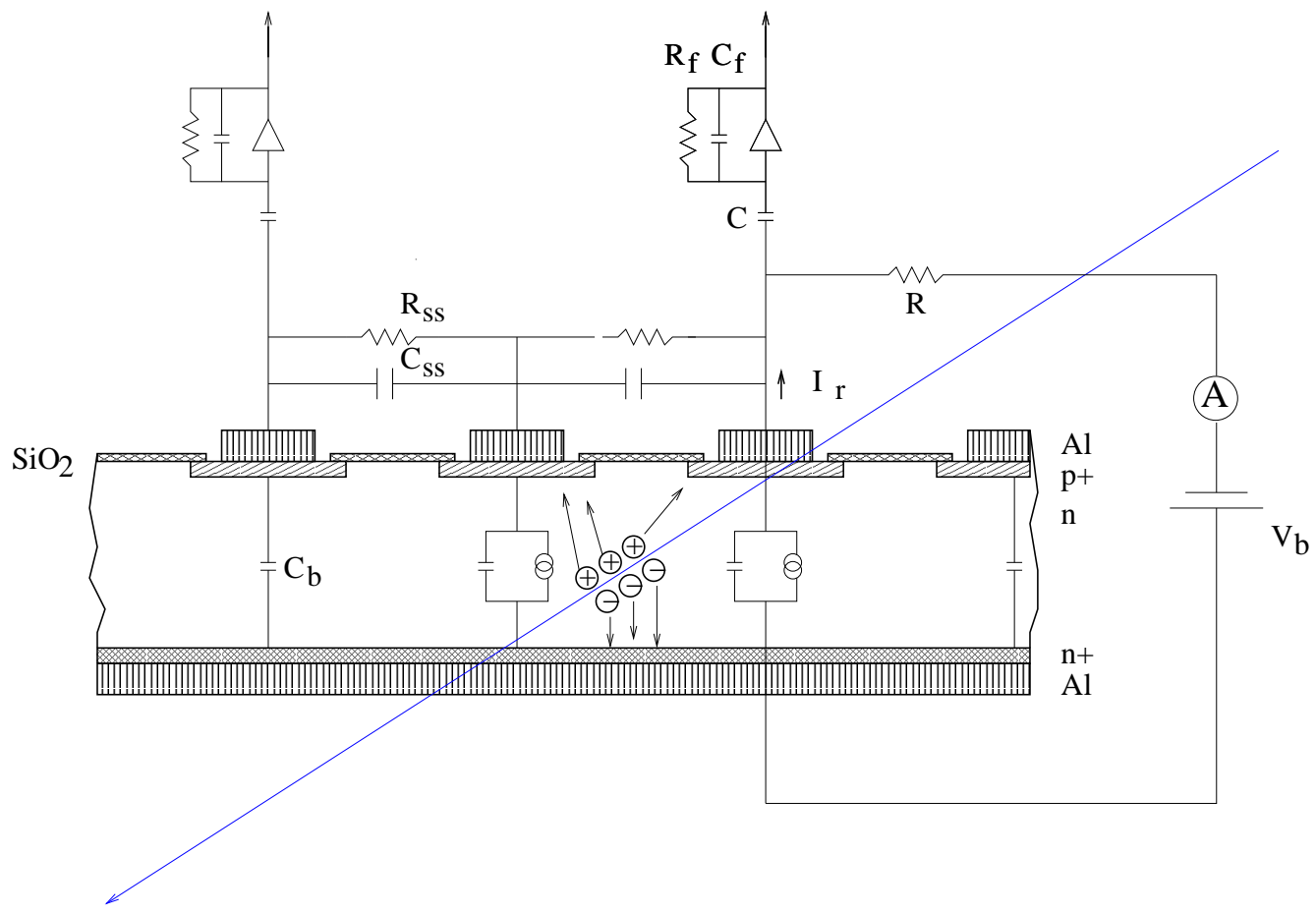

Figure D.6: Electronic circuit superimposed over a cross section of the sensor. The detector's bulk is represented as a capacitor in the circuit. When an incident particle ionizes the silicon, the detector's bulk circuit is transformed into a current source in parallel, to account the creation of electron-hole pairs. In a capacitive coupling (made by capacitor $C$ ) design, only the AC part of the detector reverse bias current $I_{r}$ reaches the readout and amplifying electronics. The DC part goes into a bias circuit, here shown as a simple resistor $R$. Intermediate "floating" electrodes can be used to reduce the effective readout pitch. In this case, the signal current induced on the floating electrodes is transferred to the readout amplifiers by intermediate capacitive dividers formed by the strip-to-strip capacitance $C_{s s}$ and the backplane capacitance $C_{b}$. 



\section{Bibliography}

[1] Fermilab Beam Division, http://www-bd.fnal.gov

[2] Fermilab Beam Division, “Run II Handbook”, http://www-bd.fnal.gov/runII/index.html

[3] R. Blair et al.,

"The CDF-II detector: Technical Design Report", FERMILAB-PUB-96/390-E (1996).

[4] A. Still et al.,

“SVX-II: CDF Run II Silicon Tracking Projects",

Nucl. Instrum. Methods, A 447, 1-8 (2000).

[5] T.Affolder et al.,

"COT Central Outer Tracker",

Nucl. Instrum. Methods, A 526, 249 (2004).

[6] CDF Tracking Group private conversation;

See also http://www-cdf.fnal.gov/internal/detectors/parameters.html

http://www-cdf.fnal.gov/cdfsim/validation/cot/cotVal.html

[7] D. Acosta et al.,

"A Time-Of-Flight Detector in CDF-II",

Nucl. Instrum. Methods, A 518, 605-608 (2004).

[8] L. Balka et al.,

"The CDF central electromagnetic calorimeter",

Nucl. Instrum. Methods, A 267, 272 (1988).

[9] S. Bertolucci et al.,

"The CDF central and endwall hadron calorimeter",

Nucl. Instrum. Methods, A 267, 301 (1988). 
[10] M. Albrow et al.,

"The CDF plug upgrade electromagnetic calorimeter: test beam results",

Nucl. Instrum. Methods, A 480, 524 (2002).

[11] G. Ascoli et al.,

"CDF central muon detector",

Nucl. Instrum. Methods, A 268, 33 (1988).

[12] K. Byrum et al.,

"The CDF forward muon system",

Nucl. Instrum. Methods, A 268, 46 (1988).

[13] D. Acosta et al.,

"The CDF Luminosity Monitor",

Nucl. Instrum. Methods, A 461, 540 (2001).

[14] D. Acosta et al.,

"The Performance of the CDF Luminosity Monitor",

Nucl. Instrum. Methods, A 494, 57 (2002).

[15] F. Abe et al.,

"Measurement of the anti-proton proton total cross section at $\sqrt{s}=546 \mathrm{GeV}$ and 1800

GeV",

Phys. Rev. D 50, 5550 (1994).

[16] S. Klimenko, J. Konigsberg and T. Liss,

"Averaging the inelastic cross-section measured by CDF and the E811 experiments", Fermilab-FN-0741 (2003).

[17] S. Jindariani et al.,

"Luminosity Uncertainty for Run 2 up until August 2004", CDF/ANA/7446 (2005).

[18] H.J. Frisch and P.J. Wilson,

"Trigger tower organization and summing in $\eta-\phi$ space for Run II and beyond", CDF/DOC/TRIGGER/CDFR/2045 (2001).

[19] The XFT System Webpage, http://www.physics.ohio-state.edu/ hughes/xft/

[20] The XTRP Webpage, http://web.hep.uiuc.edu/engin/cdf/xtrp/

[21] I. Vila,

"Performance and First Physics Results of the SVT Trigger at CDFII", arXiv:hep-ph/0307165 (2003). 
[22] The Trigger and Datasets Working Group,

"Run II Trigger Table and Datasets Plan", CDF/PHYS/TRIGGER/CDFR/4718 (2001).

[23] Furgeri et al. Radiation Damage Effects on CMS Sensors: Quality Assurance and Irradiation Tests. IEEE Transactions on Nuclear Science, Vol. 51, NO. 6, December 2004

[24] M. Moll "Radiation damage in silicon particle detectors." M.S. Thesis Univ. Hamburg. Hamburg, Germany 1999.

[25] M. Moll "Radiation damage in silicon particle detectors." M.S. Thesis Univ. Hamburg. Fig. 5.1. Hamburg, Germany 1999.

[26] K.G. McKay, Phys. Rev. 84 (1951) 829.

[27] G. Goldhaber et al., Phys. Rev. Lett. 37 (1976) 255.

[28] G. B. Chadwick, Proc. Int. Europhysics Conf. on High Energy Physics, Brighton, July 1983 (Rutherfors Appleton Lab., 1983) p. 330

[29] M.I. Adamovich et al. (Photon-Emulsion collaboration), Phys. Lett. 99B (1981) 271.

[30] C. Damerell, Nucl. Instrum. Meth. in Phys. Res. 226, 57 (1984) 26-33.

[31] N. Ellis and A. Kernan, Phys. Rept. 195 (1990) 23.

[32] The CDF Collaboration, "Measurement of b-hadron lifetimes in decays to J/P", http://www-cdf.fnal.gov/physics/new/bottom/091217.blessed-JpsiX4.3/jpsix.html

[33] http://arxiv.org/abs/0912.3566

[34] F. Abe et al., Phys. Rev. Lett. 68 (1992) 3403.

[35] M. Paulini, Czech. J. Phys. 53 (2003), Suppl. A.

[36] D. Acosta et. al ., Phys. Rev. Lett 91 (2003) 241804 [hepex/0307080]

[37] CDF Col., http://www-cdf.fnal.gov/physics/new/bottom/090813.blessedBsd2mumu//welcome.html

[38] CDF Col., http://www-cdf.fnal.gov/physics/new/bottom/080228.blessed-d0-mumu

[39] First precision measurement of $\Delta m_{s}$, A. Abulencia et. al., Phys. Rev. Lett. 97 (2006) 062003

[40] Observation of $B_{s}-\bar{B}_{s}$ oscillations, A. Abulencia et. al., Phys. Rev. Lett. 97 (2006) 242003 
[41] $B_{s}$ Lifetime Difference and Mixing at the Tevatron, S. Hansmann-Menzemer, CDF/PUB/BOTTOM/PUBLIC/8670, (2007)

[42] N. F. Mott, "The basis of the electron theory of metals in special reference to the transition metals", Proc. Phys. Soc., vol. A61, p.416, 1949.

[43] N. F. Mott, "On the transition to metallic conduction in semi-conductors", Can. J. Phys., vol. 34, p. 1356, 1956.

[44] P. W. Anderson "Absence of diffusion in certain random lattices", Phys. Rev., vol. 109, p. $1492,1958$.

[45] P. P. Edwards and M. J. Sienko, "Universality aspects of the metal-nonmetal transition in condensed media”, Phys. Rev., vol. B17, p. 2575, 1978.

[46] Niemeyer, L., Pietronero, L., Wiesmann, H., "Fractal dimension of dielectric breakdown", Physical Review Letters 52 (1984), 1033-1036.

[47] S. T. Lai, "The Mott transition as a Cause of Anomalies on Spacecraft", IEEE Transactions on Plasma Science, vol. 28, No. 6, (Dec. 2000)

[48] A. R. Frederickson, "Radiation induced dielectric charging", in Space Systems and their Interactions with the Earth's Space Environment, H. B. Garret and C. P. Pike, Eds: AIAA Press, 1980, pp.386-412

[49] S. T. Lai, "A Mechanism of deep dielectric charging and discharging on spacecraft", in Proc. IEEE International Conf. Plasma Sci., Raleigh, N.C., 1988.

[50] N. F. Mott, "Metal-Insulator Transitions". London, U.K.: Taylor and Francis, 1974.

[51] H. Kimimura and H. Aoki, "The Physics of Interacting Electrons in Disordered Systems”. Oxford: Clarendon Press, 1989.

[52] N. F. Mott, "Nobel prize lecture", in Nobel Lectures, S. Lundqvist, Ed. Singapore: World Scientific, 1977.

[53] C. Kittel, "Introduction to Solid State Physics", 5th ed. Wiley, New York, 1976.

[54] L. Li et al. "Influence of traps on charge transport in organic semiconductors", SolidState electronics 51 (2007), 445-448.

[55] L. Li, G. Meller, H. Kosina, "Temperature and field-dependence of hopping conduction in organic semiconductors", Microelectronics Journal (2007), 38 (1), pp. 47-51

[56] A. Miller, E. Abrahams "Impurity conduction at low concentrations", Phys. Rev. 1960, 120(3), 745-55 
[57] M. Sahimi, Applications of Percolation Theory. London, UK. Taylor and Francis 1994.

[58] V. Ambegaokar et al. Phys. Rev. B 1971 4(8):2612-20

[59] H. Fishchuk, H. Bassler Phys. Rev. B 2002;66:2052081-20520812.

[60] C. Kittel "Introduction to Solid State Physics", Chap. 6. Ed. Wiley and Sons, 1976.

[61] D. Acosta, et al., (CDF Collaboration), Phys. Rev. D 71, 032001(2005).

[62] C.S. Hill, et al., Nucl. Instr. and Meth. A 511 (2003) 118.

[63] A. Sill, et al., Nucl. Instr. and Meth. A 447 (2000) 1.

[64] W. Ashmanskas, et al., Nucl. Instr. and Meth. A 518 (2004) 532.

[65] A. Affolder, et al., Nucl. Instr. and Meth. A 453 (2000) 84.

[66] S. Worm, et al., Nucl. Instr. and Meth. A 549 (2005) 126.

[67] A. Boveia, Status and performance on the CDF Run II silicon detector, PoS HEP2005, 2005 , p. 377.

[68] R.J. Tesarek, et al., Nucl. Instr. and Meth. A 514 (2003) 188.

[69] S. D’Auria, et al., Nucl. Instr. and Meth. A 513 (2003) 89.

[70] H. Spieler, Semiconductor Detector Systems, Oxford Pub. 2005. P.16.

[71] G. Lutz, Semiconductor Radiation Detectors: Device Physics. Ed. Springer, 1999. Chapter 3, p.43.

[72] CDFII Collaboration Technical Design Report, FERMILAB-PUB-96/390-E.

[73] T.K. Nelson for the CDF II Collaboration, The CDF Layer 00 Detector, FERMILABCONF-01/357-E.

[74] Huseman, Ulrich. Nucl. Instr. and Meth. A, Volume 569, Issue 1, p. $65-68$.

[75] M. Garcia-Sciveres, et al., Nucl. Instrum. Meth. A435 (1999) 58-64.

[76] M. L. Chu, et al., Nucl. Instrum. Meth. A541 (2005) 208-212.

[77] M. Moll, "Development of Radiation-Hard Sensors for Very High Luminosity Colliders: CERN RD-50 Project” Nucl. Instrum. Meth. A511 (2003) 97-105.

[78] P. Dong et al., “An Analysis of Bias Currents in SVX II”, CDF note 8219, Nov. 20, 2006. 
[79] D. Amidei et al. "The silicon vertex detector of the collider detector at CDF", Nucl. Inst. and Meth. A350 (1994), pp. 73-130.

[80] R. J. Tesarek, "A measurement of the radiation environment in the CDF tracking volume", Nucl. Inst. and Meth. A514 (2003), pp. 188-193.

[81] V. Eremin et al., "The origin of double peak electric field distribution in heavily irradiated silicon detectors", Nucl. Inst. and Meth. A476 (2002), pp. 556-564.

[82] G.Lindstrom, "Radiation damage in silicon detectors", Nucl. Inst. and Meth. A512 (2003), pp. 30-43.

[83] M. Swartz et al., "Type inversion in irradiated silicon: half a truth", arXiv:physics/0409049v2 13 sep 2004.

[84] V. Chiochia, "Simulation of Heavily Irradiated Silicon Pixel Sensors and Comparison with Test Beam Measurements”, arXiv:physics/0411143v2 4 may 2005.

[85] M. Moll et al., "Leakage current of hadron irradiated silicon detectors-material dependence", Nucl. Inst. and Meth. A426 (1999), pp. 87-93.

[86] S. Saramad., "A new explanation for some open questions of hadron irradiated silicon detectors", Nucl. Inst. and Meth. A510 (2003), pp. 101-106.

[87] Niemeyer, L., Pietronero, L., Wiesmann, H., "Fractal dimension of dielectric breakdown,” Physical Review Letters 52 (1984), pp. 1033-1036.

[88] Andersen et al., "The Port Card for the Silicon Vertex Detector Upgrade pf the CDF at Fermilab"

[89] Technical Information Manual, MOD. A 509H. NPO: 00109/99:A509Hx.MUTx/00

[90] J.S. Kapustinsky et al., "Radiation damage effects on the silicon microstrip detector in E789 - A fixed target experiment at fermilab”, Nuclear Physics B - Proceedings Supplements. Volume 32, May 1993, Pages 425-430.

[91] E. Spenke, “Elektronische Halbleiter”, 2nd ed., Springer, Berlin (1965).

[92] Vasiliescu, A., (1998) et. al. "Fluence normalization based on NIEL Scaling hypothesis", 3rd ROSE Workshop, DESY Hamburg, 12-14 February 1998, DESY Proc. 1998-2.

[93] Wunstorf, R. (1992): "Systematische Untersuchungen zur Strahlenresistenz von Silizium-Detektoren fuer die Verwendung in Hochenergiephysik-Experimenten", Ph. D. thesis, Universitaet Hamburg, 1992. 
[94] Fretwurst, E., Feick, H.(1994) et al. "Reverse annealing of the effective impurity concentration and long term operational scenario for silicon detectors", Nucl. Inst. and Meth. A288 (1994), pp. 1-12.

[95] Chilingarov, A., and Sloan, T.(1997b). “Operation of heavily irradiated silicon detectors under forward bias”. Nucl. Inst. and Meth. A399 (1997), pp. 35-37.

[96] R. Tesarek, S. D’Auria, A. Hocker, K. Kordas, S. McGimpsey, S. Worm. "A Measurement of the Radiation Environment in the CDF Tracking Volume". CDF/PUB/SECVTX/PUBLIC/6060. Pub. Info: Published Proceedings 4th International Conference on Radiation Effects on Semiconductor Materials Detectors and Devices (RESMDD02), Florence, Italy, July 10-12, 2002. FERMILAB-CONF-02 189-E. 
